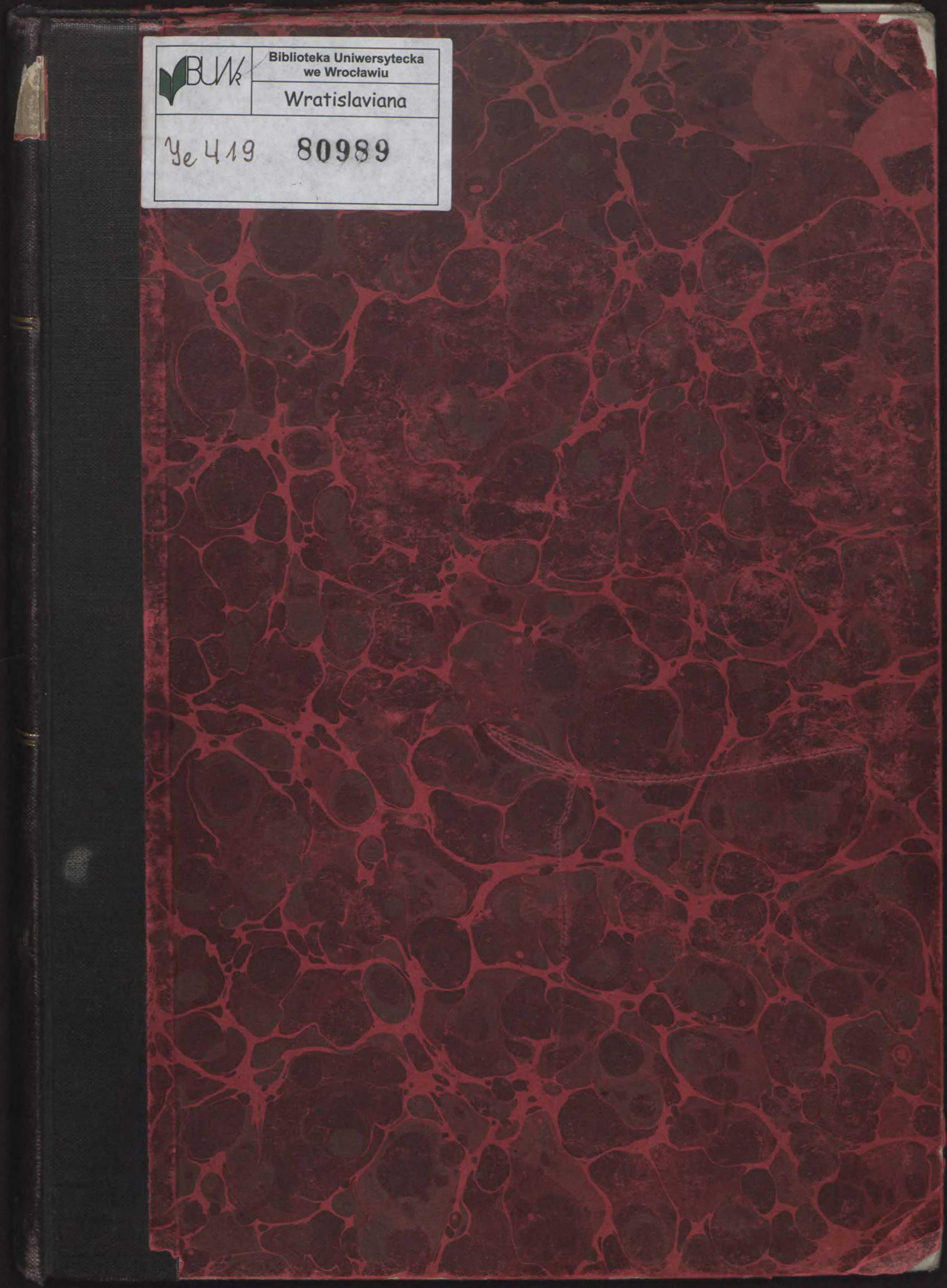




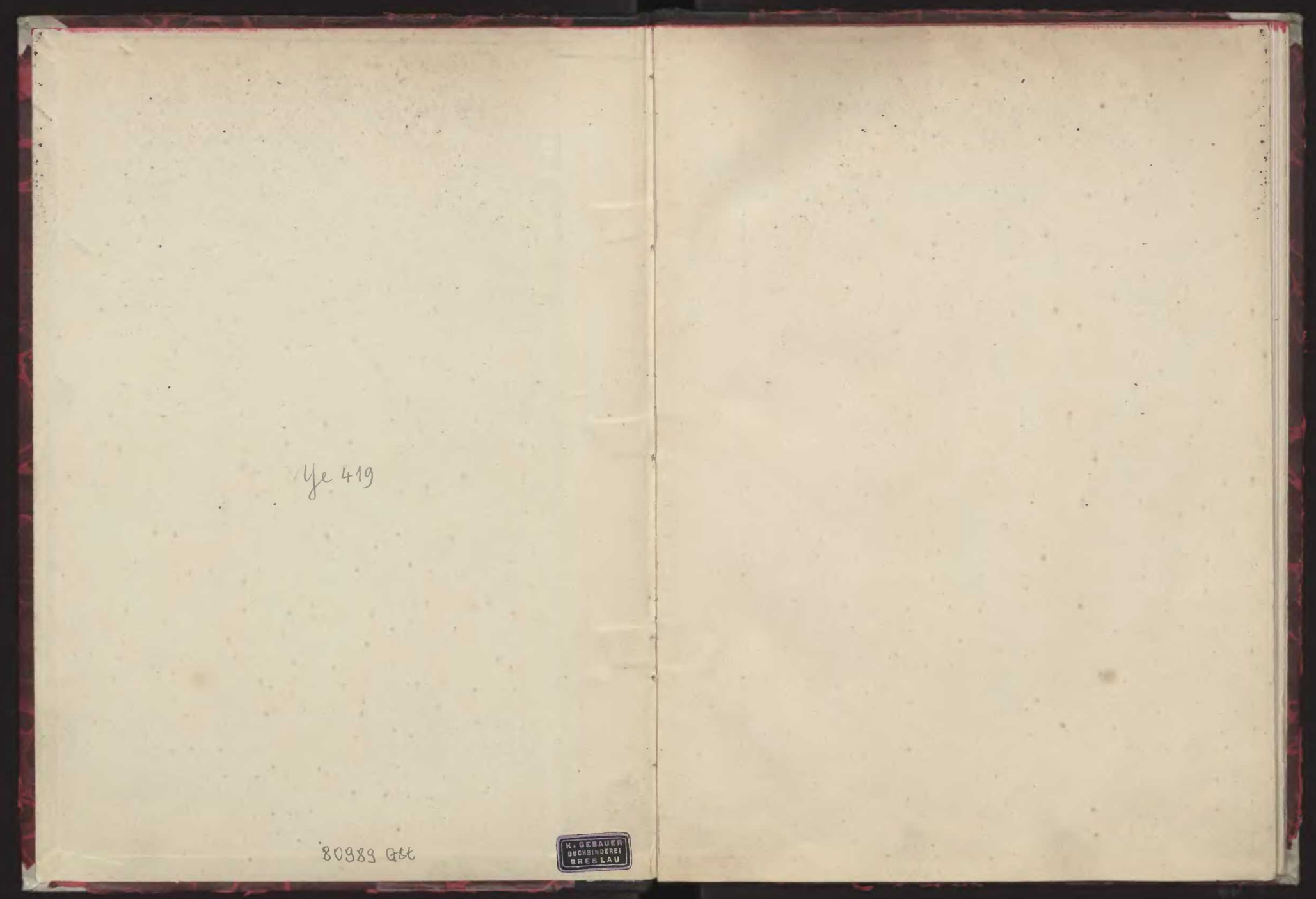




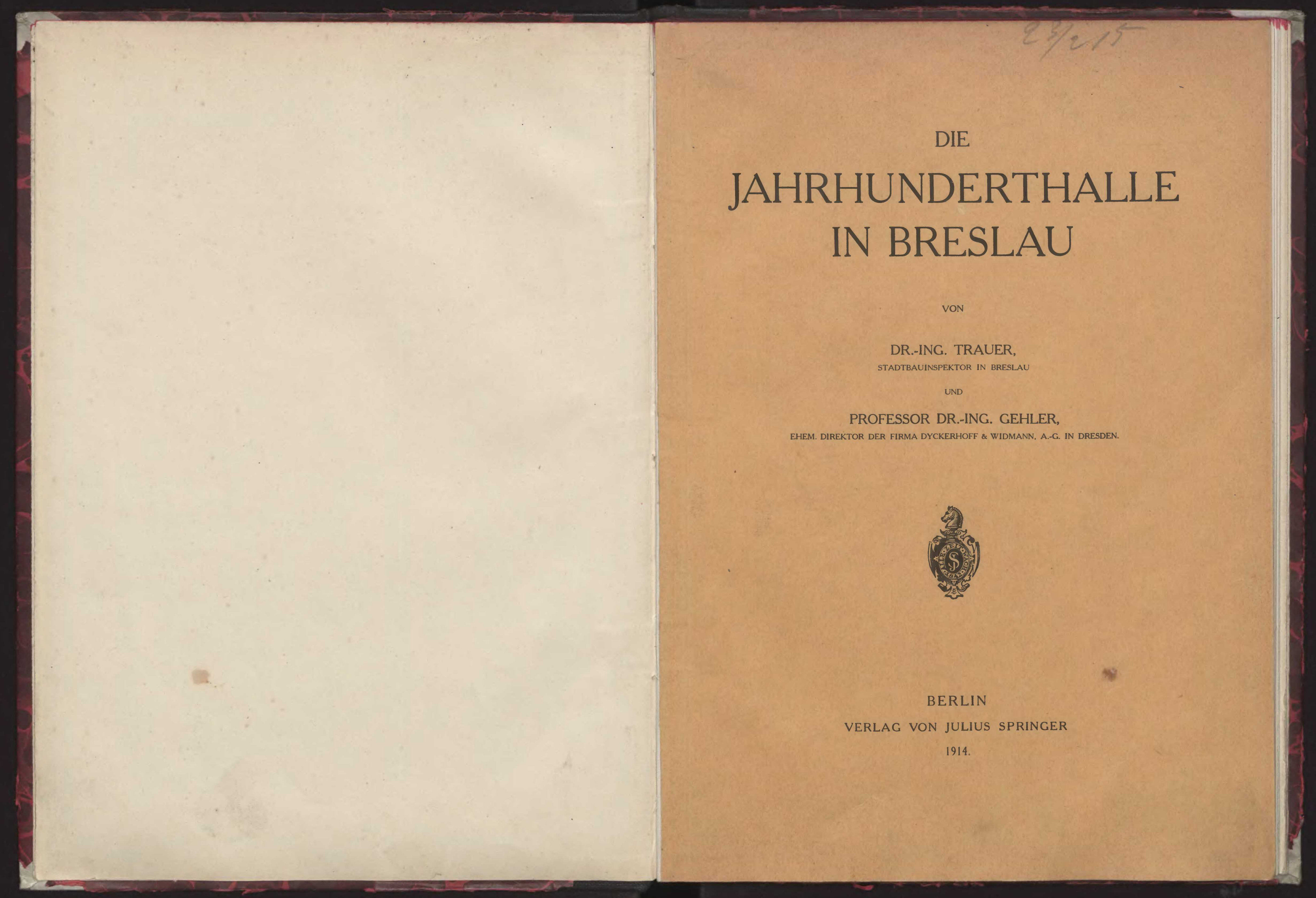




\section{DIE JAHRHUNDERTHALLE IN BRESLAU}

VON

DR.-ING. TRAUER, TADTBAUINSPEKTOR IN BRESLAU

UND

PROFESSOR DR.-ING. GEHLER EHEM. DIREKTOR DER FIRMA DYCKERHOFF \& WIDMANN, A.-G. IN DRESDEN

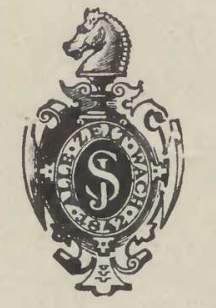

BERLIN

VERLAG VON JULIUS SPRINGER

1914. 

in Dresden.

Zur Erinnerung an die ruhmreiche Erhebung Mittelbau umschließen einstöckige Ringbauten, des deutschen Volkes zum Kampfe gegen die welche die Nebenräume aufnehmen und im übrigen napoleonische Knechtschaft im Jahre 1813 veran- Ausstellungszwecken dienen sollen. Die Eingänge
staltet die Stadt Breslau eine Ausstellung, welche

die Freiheitskriege im Rahmen der Kunst und
Kultur ihrer Zeit darKultur ihrer Zeit dar-
stellen soll. Von den
inmitten des prächticen Scheitniger Parkes nach einem großzügigen Plane errichteten Ausdestungsebauden ist große Festhalle. Sie ist zugleich als dauerndes Denkmal zur Erinnerung dacht und soll eineStätte für szenische, musikalische und sportliche Veranstaltungen sowie fur Feste un

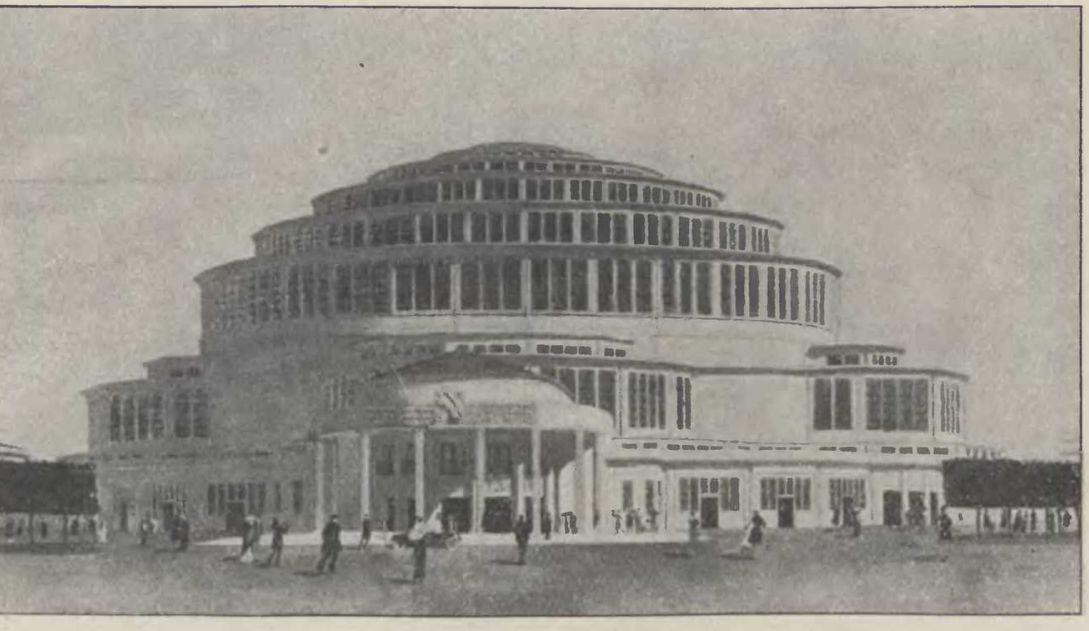
gresse werden.
Die Festlat FIs. 1. Fertige Halle

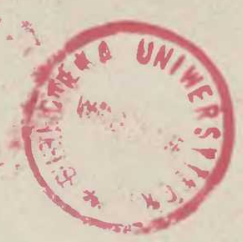
Berg. Sie besteht aus einem zentralen Kuppel- seitige ist als Haupteingang ausgebildet worden bau von $42 \mathrm{~m}$ Höhe und $65 \mathrm{~m}$ lichtem Durch- und enthält über der Eintrittshalle einen Empfangs

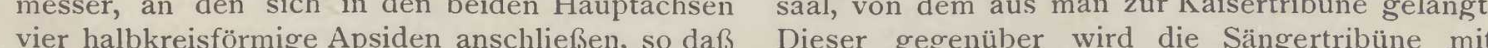
ein von konstruktiven Einbauten freier Raum von einer 200-stimmiren Ororel, dem größten bisherige $95 \mathrm{~m}$ grosster Erstreckung in den Hauptachsen Werke erbaut, wăhrend in den anderen beiden und von rd. $5500 \mathrm{qm}$ Fläche geschaffen ist. Diesen Apsiden $5 \mathrm{~m}$ weit auskragende Balkone ange- 

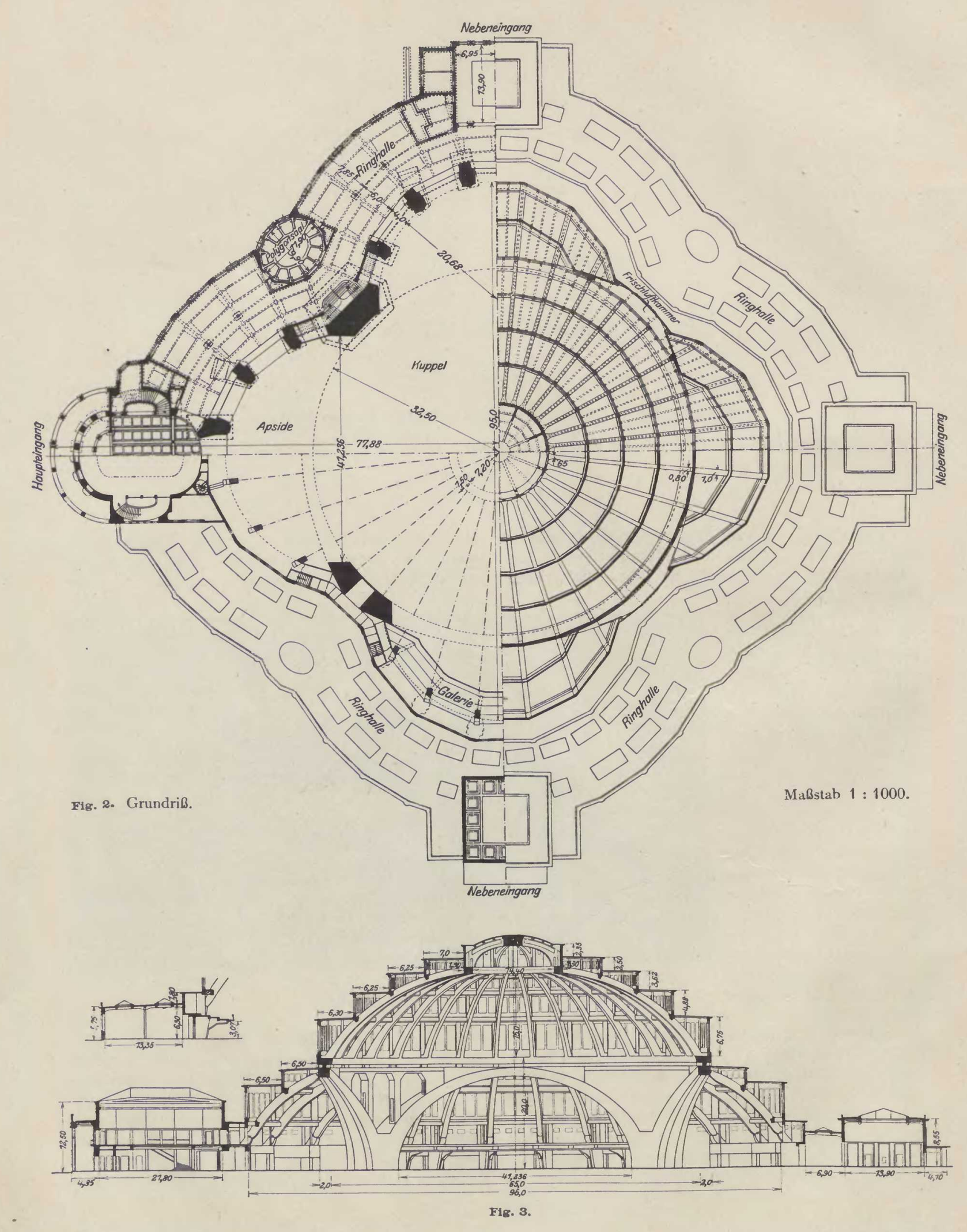

ordnet sind. Für die szenischen Vorführungen Dyckerhoff \& Widmann, A.G. in Dresden, der auferdem noch die konstruktive Durd beseitigt werden kann. Die Halle bietet Sitzplätze führung der einstöckigen Ringbauten ist durch für fast 6000 Personen oder Stehplätze für an- die Firma Lolat Eisenbeton, A. G. in Breslau bescheinung
Formen ab. Die Raumumschließsung wird nicht Es stand von vornherein fest, daß bei den

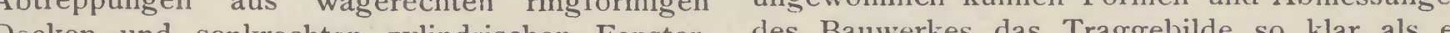
wänden. Da diese senkrechten Fensteffïchen möolich war, auscrebildet werden mußte, und da - dem Verschmutzen und der Verdunkelung durch ungünstig wirkende statische Unbestimmtheite Schnee nicht ausgesetzt sind, so sind die Licht- durch Einfügen von Gelenken, Trennfugen und verhaltnisse sehr gute. Das gleiche Motiv durch anmilche Maßnahmen ausgeschaltet iverden ist auch

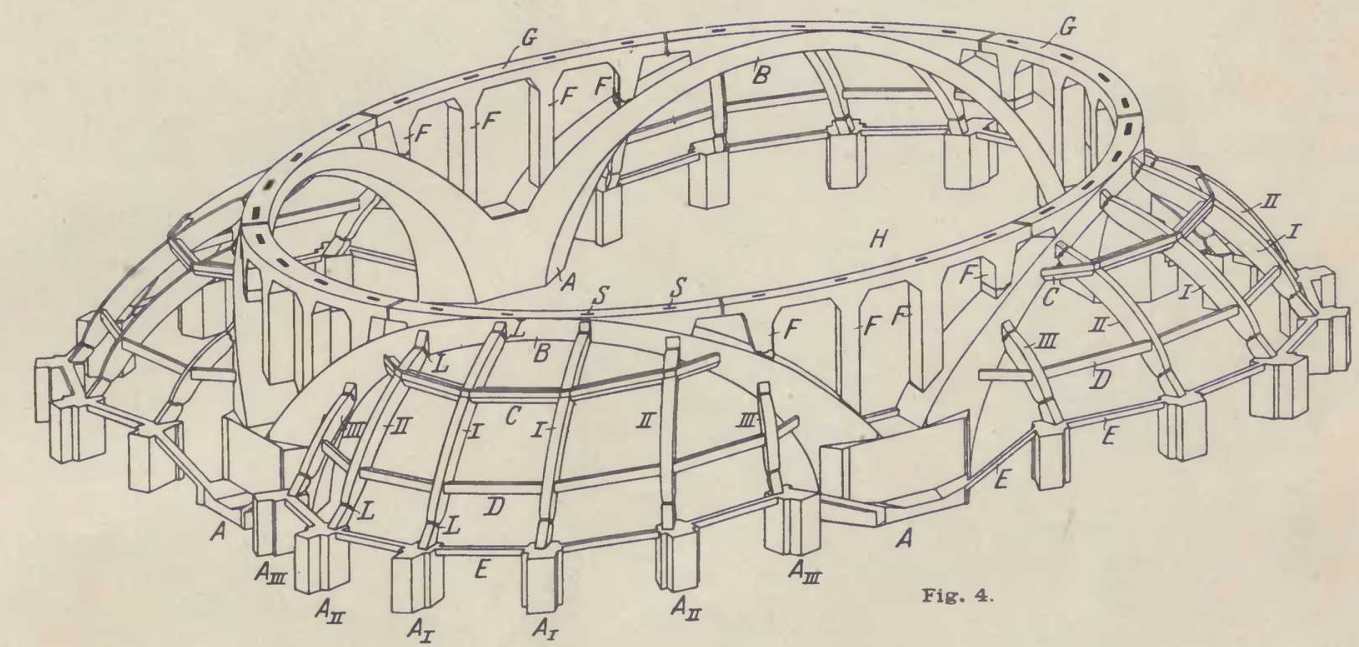

TEIL I.

Von Dr.-Ing. Trauer.

Wiewohl der raumkünstlerische Gedanke und Architekten ist, so mußte doch von Anfang an neben die architektonische Bearbeitung die in-
genieurtechnische Durcharbeitung treten. Im Gegensatze zu der üblichen Gepflogenheit bei Hochbauten wurde bereits vor der Ausschreibung der Arbeiten von der Bauverwaltung eine umfangreiche Berechnung für die Haupttraggebilde ganzen festzulegen, die Ausführbarkeit zu erweisen und brauchbare, einheitliche Unterlagen
für die Ausschreibung zu schaffen. Die endgültigen Berechnungen der Haupttragteile sind
dann im wesentlichen nach den Richtlinien der

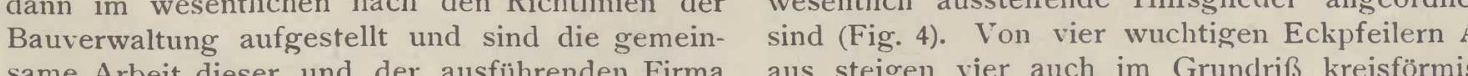

19 m hohen Kuppelunterbau. Das Kuppeltragwerk wird gebildet aus 32 halben Rippen, die inten gegen einen Zugring stuttzen. Die Kuppel ht auf dem Unterbau vermittels 32 radial beeglicher Stelzenlager mit Tangentialkippvorrich-

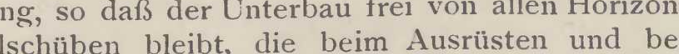
ärmeänderung der Kuppel im Falle einer festen endung mit dem Unterbau auftreten würden. Wie Windkräfte werden infolge der radialen 列, also der Richtung übertragenn, in der dieser ie größte Widerstandsfähigkeit gegen wageKräfte besitzt. Der Kuppelunterbau ist ein atisch äulserst schwieriges Gebilde und gegen

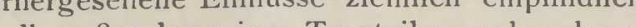


geführte Hauptbogen B von 41,236 $\mathrm{m}$ Weite und handen, die aber an der Übertragung der Haupt(6)

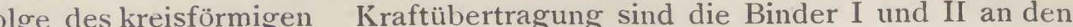
Grundrisses auftreten, in jeder Apside gegen vier oberen und unteren Kämpfern mit Stahllugel-

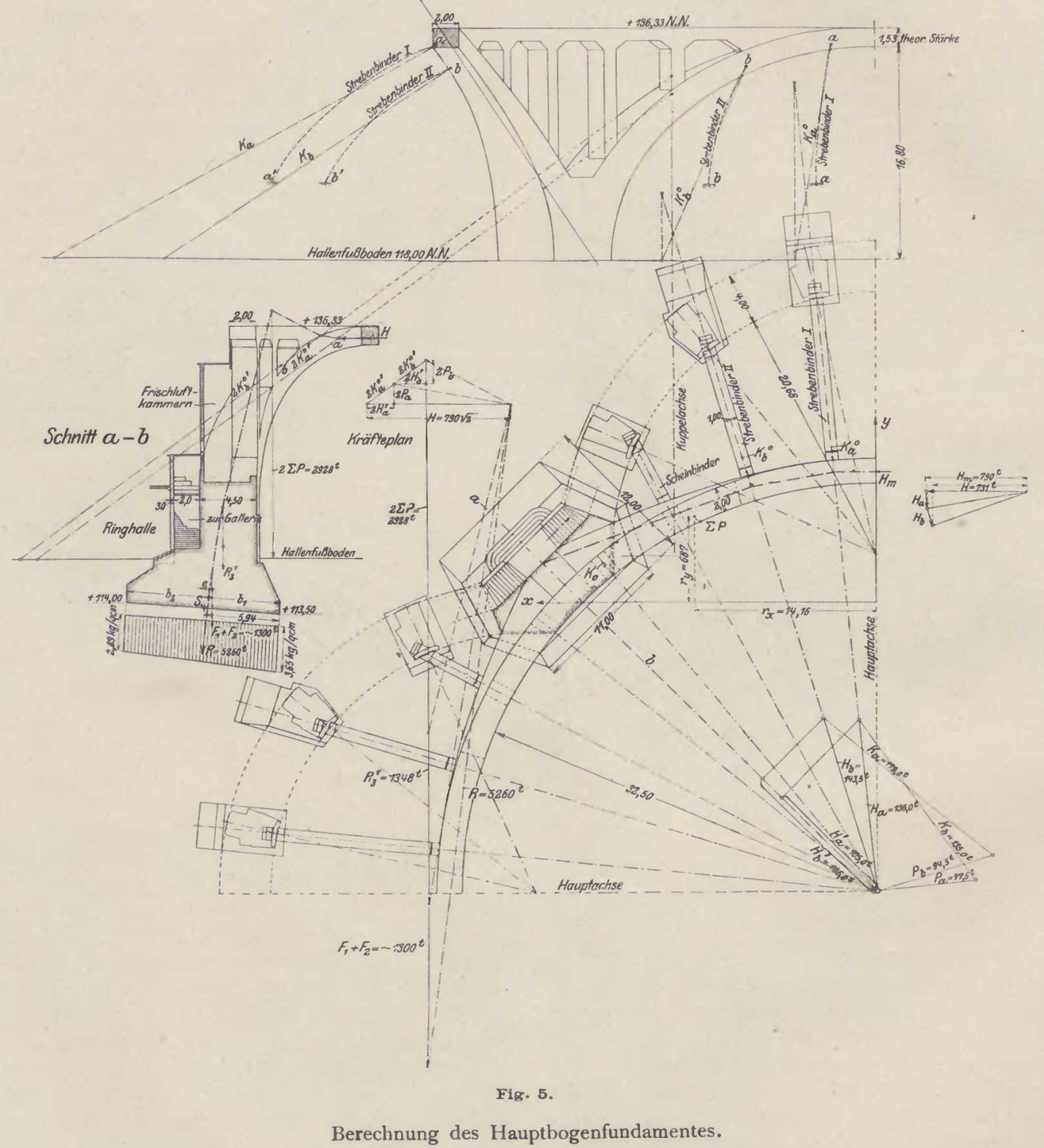

Strebenbinder I und II, welche in straffer, wenig lagern L versehen. Die Knicksicherheit in seitlicher gehronter Linie nach den Strebepfeilern Ar ang Kinter des Hauptbogens und ibrem Eigengewichte noch der Firma eingefügt worden sind, erhöht. Un die Lasten der Apsidenaufbauten aufzunehmen mittelbar unter den Füßen der Strebenbinder is haben. Außer diesen tragenden Strebenbindern in der Hohe des Galerieumganges noch ein Z sind aus architehtonischen Granden

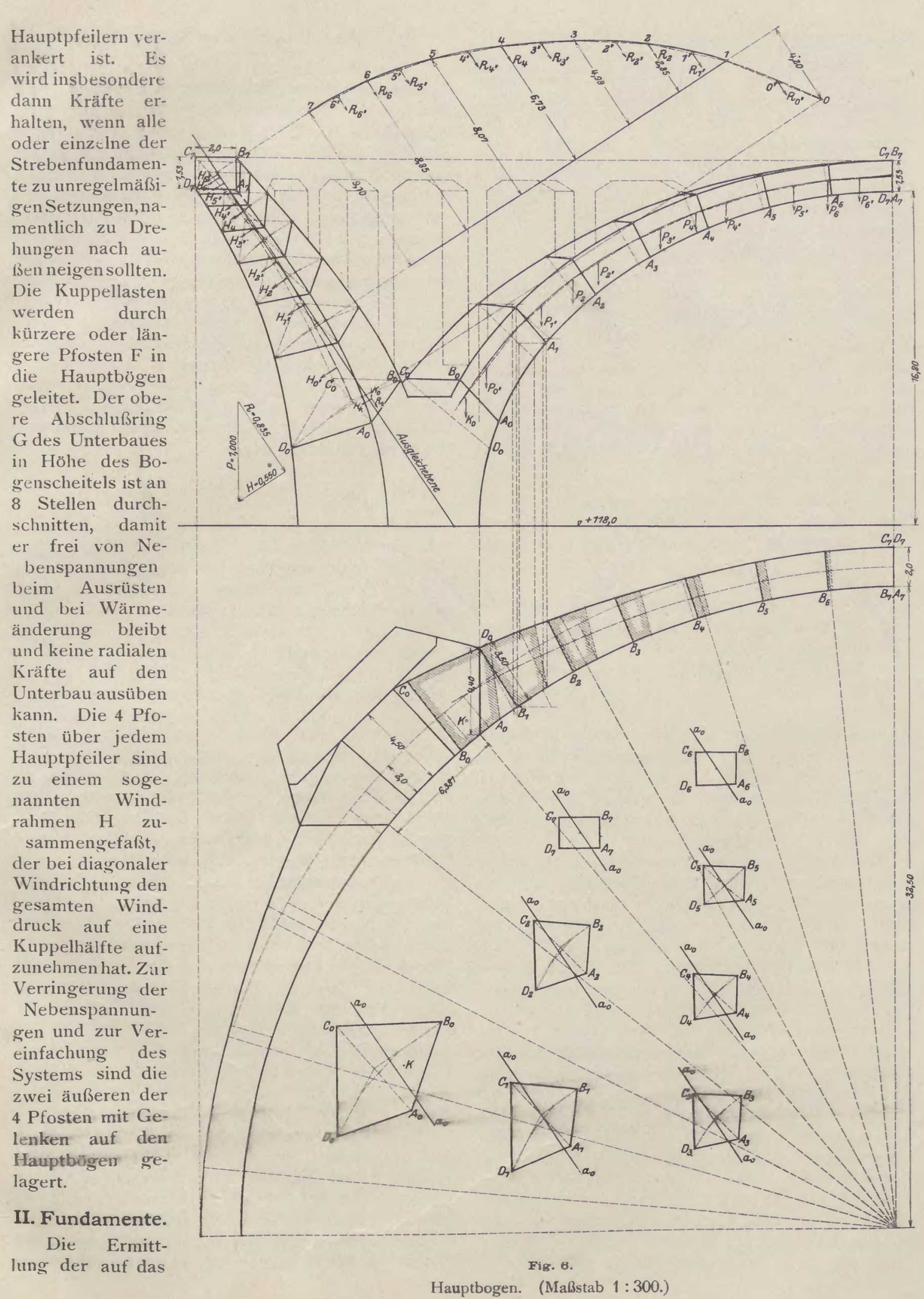


$-8-$

Hauptfundament wirkenden Kräfte führt
zu der Aufgabe, beliebig gerichtete Kräfte im
Raume zu einer resultierenden Kraft und einem resultierenden Kräftepaare zusammensymmetrische Lage aller Kräfte wesentlich vereinfacht. Führt man für einen Quadranten die beiden Hauptachsenschitte, so wirkt in einem bestimm stimmte Bogenkraft $\mathrm{H}$, ferner wirken auf jeden Hauptbogen in den oberen Gelenkpunkten a un b der Strebenbinder die nach Gräe und Richtung ebenfalls be kand endich die senkrechten Lasten $\mathrm{P}$, deren $\mathrm{Re}$

sultante $\sum \mathrm{P}$ ist (Fig. 5). Je zwei symmetrisch gelegene Kräfte lassen sich nun zu einer in der Diagonalebene liegenden Resultante zusammen-
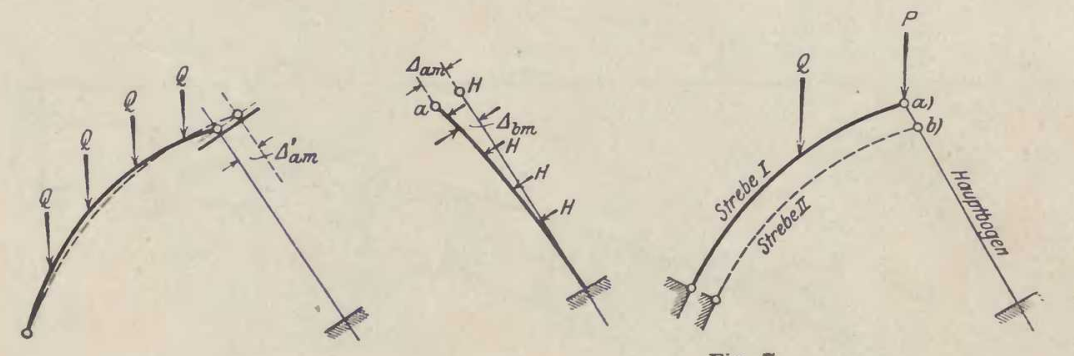

in einer Ebene zu der Gesamtresultante $R$ zusamder Größtwert, der bei Eigengewicht und Wärmezunahme $\left(+7,5^{\circ}\right)$ auftritt, eingeführt. Als Bodedrücke ergeben sich 3,65 und $2,89 \mathrm{~kg} / \mathrm{q} \mathrm{cm}$, als eine nahezu zentrische Belastung.

bietet insofern einige Besonderheiten, als die Achse der Pfeiler aus architektonischen Gründe nicht mit der Kraftebene der Strebenbinder. $z$ sammenfallt, wathrend die Hauptrichtung der Fberparallel ist. Die Bodendrücke der einzelnen Fund mente bewegen sich $z$ wischen 1,4 und $3,3 \mathrm{~kg} / \mathrm{qcm}$. Es war vorgeschrieben, daf der grốste Bodenmindestens noch $1 / 3$ des größtsten betragen und daß die Resultante um höchstens $10^{\circ}$ von der Normalen zur Fundamentsohle abweichen sollte. Die Eiseneinlagen des bereits erwähnten Zugbandes
in der Höhe des Galerieumganges $\left(+4,50^{\circ}\right.$ ) sind so stark bemessen, daß es von dem Schube de so viel aufnehmen kann, daß di Sohlenschwerpente

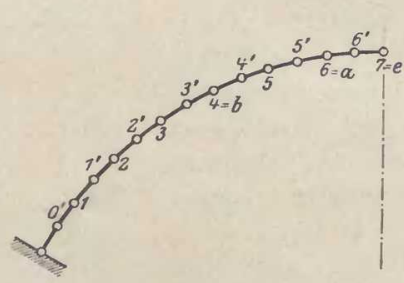

III. Hauptbogen und Strebenbinder. Der Hauptbogen wurde zur angenäherten er Stitzlinie konstruiert Die Strebenbint nac Itten dann Horizontalschübe von solcher Gröbe, aßß die Stützlinie des Hauptbogens im Grundriss polygonal unter möglichster Anlehnung an die ogenachse verläuft. Die Horizontalkraft jedes usetzen, und es ist ferner in dessen oberen

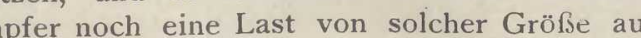
en Strebenbinder zu rechnen, daß die Stützlini

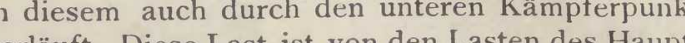
作 chub. Unter Annahme nicht zu hoher Betonannungen wurden hiernach die Abmessunge les Hauptbogens und der Strebenbinder festge-

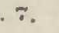

Zeichnet man einen Achsenschnitt durch die Halle (Fig. 6) und projiziert man auf diesen den auptbogen mit den einzelnen Quersch silten, so is in liegend angesehen werden kann, vor allem in dem Teile des Hauptbogens, der infolge seiner geringeren Querschnitte zu den elastischen Formanderungen den grobten Beitrag liefert. Zu dieser Ausgleich der 政 blene berücksichtigt worden. Man kann jetzt den Hauptbogen als eimen ebenen beiderseits eingeSpanten Stabbogen anselone P werden nun zerlegt in Kräfte in der Ausgleichebene, $\mathrm{R}=0,835 \mathrm{P}$ und in solche senkrecht $\mathrm{zu}$ dieser, $\mathrm{H}=0,55 \mathrm{P}$. Auch die Auflagerdrücke der Strebenbinder werden in dieser Weise zerlegt. Horizontalschübe oder deren zur Ausgleichebene senkrecht stehenden Komponenten $\mathrm{X}_{\mathrm{a}}$ und $\mathrm{X}_{\mathrm{b}}$ lassen sich nunmehr als statisch unbestimmt Grốfsen eines senkrecht za seiner mbene durch

$-9$

tatisch unbestimmten beiderseits eingespannten ogens auffasse

1. Einwirkung der Kräfte senkrecht zur

Denkt man sich die Verbindung des Hauptbiegen die Lasten $\mathrm{H}$ den Hauptbogen aus der Ausgleichebene und zwar in den Punkten $6=$ und $4=-b$ and de

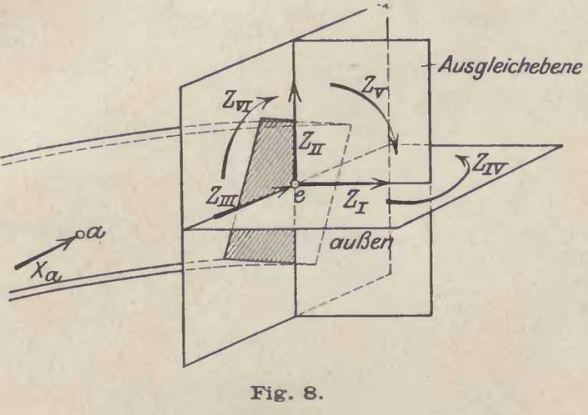

Die Lasten $Q$ der Strebenbinder verschieben deren obere Endpunkte $\mathbf{a}$ und $\mathbf{b}$ im statisch be-
stimmten Hauptnetz auf senkrecht zur Ausgleichebene gefülrten Auflagerflächen um die MaßB $d^{\prime}$ am und $\boldsymbol{A}^{\prime} \mathrm{bm}$. Insgesamt entfernen sich also di und $A_{\mathrm{bm}}+d^{\prime} \mathrm{bm}$ im statisch bestimmten Hauptnetz. Für das statisch unbestimmte System ist die Verschiebung dieser Doppelpunkte a Streben und Bogen gleich Nu/ $\mathrm{b}$ und den Hauntbogen je 2 Kräft $X_{a}$ und $X_{b}$, welche die Entfernung
der Anschlufspunkte zu Null machen.
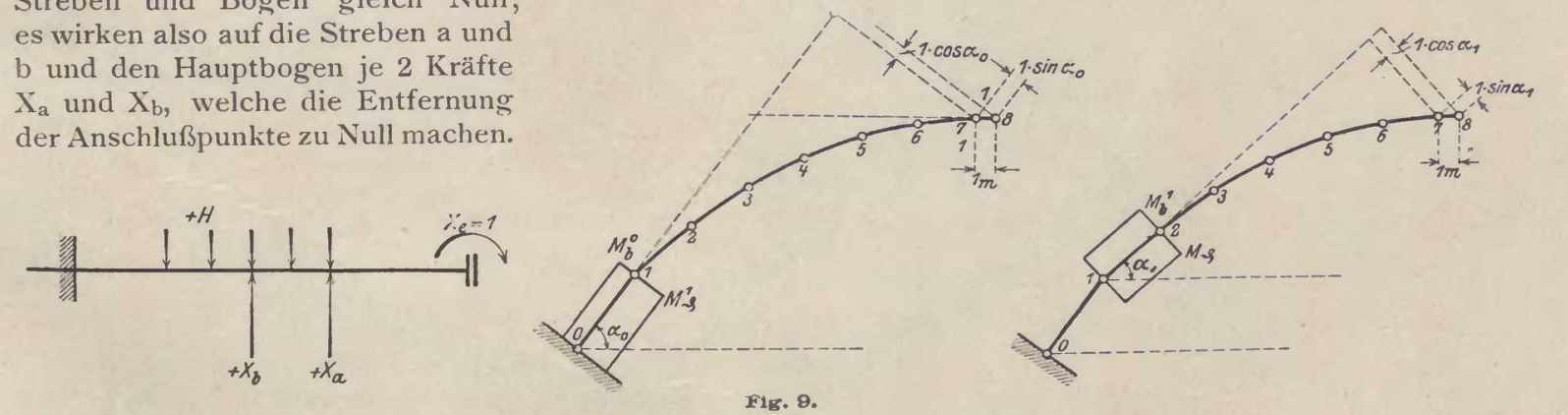

Zur Bestimmung der Verbiegungen des Haupt bogens wird in folgender Weise vorgegangen:
Der Hauptlogen wird in Mittelpunkt $7=$ durch einen Hauptachsenschnitt getremnt und es werden die in der Trennfuge wirkenden Kräfte beste $Z_{3}$ zwei Scherkräfte $Z_{H}$ und $Z_{H}$ zwe Biegunosmomente $Z_{\mathrm{IV}}$ und $Z_{\mathrm{v}}$ und ein Torsionsmoment ZVI (Fig. 8). Bei symmetrischer Belastung und Belastung ledigich durch die $X$-oder H-Krafte fallenfacht sich der K $Z_{I}, Z_{Z I I}$ und $Z_{\mathrm{VI}}$, es $f$ weiter fort $Z_{1}$ und $Z_{v}$, da bei der Geringfügiokei der Verbiegungen für jeden Punkt unter Einwirsenkrecht zur Aussleichebene in Frage komme während die Verschiebungen in der Ausgleicloment $Z_{\mathrm{iv}}=\mathrm{X}_{\mathrm{e}}$ übrig und die als äußsere Kräfte nzusehenden unbekanten $X_{\mathrm{a}}$ und $X_{\mathrm{b}}$ in $\mathrm{de}$ elenkpunkten $a$ und $b$.

rei Systeme stimmt ist:

Einwirkung der âußseren Krafte $\mathrm{H}$ und des Momentes $\mathrm{X}_{\mathrm{e}}$ (Hauptsystem),
Einwirkuny der beiden Kräfte $\mathrm{X}_{\mathrm{a}}$ und des 2. Einwirkung der beiden Kratte $X_{a}$ and des
Momentes $X_{e}$, 3. Einwirkung de Momentes $\mathrm{X}_{\mathrm{e}}$

Für jedes dieser drei Systeme werden die erschiebungen der Punkte $\mathrm{a}$ und $\mathrm{b}$ bestimmit; man erhält alsdann wesentlich weniger empfindlich gegen Ungenauig-

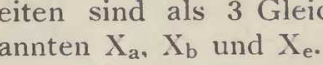

Ermittlung der Verbiegungen und Verdrehungen Zur Ermittlung von $\mathrm{X}_{\mathrm{e}}$ ist die Verbiegung des
Querschnittes $\mathrm{e}$ in der Ebene des Momentes $\mathrm{X}_{\mathrm{e}}$ a bestimmen und zwar für die 3 Falle $\mathrm{H}$. $\mathrm{X}_{\mathrm{e}}=-1 \mathrm{mt}$

Der Naxwelloche Satz lautet für die vorlie-

Eine Kraft $\mathrm{H}=1$ in einem beliebigen Punkte $m$ verbiegt den Querschnitt e in der Richtung des Momentes $\lambda_{e}$ um das gleiche Mars, wie ein Moment $\mathrm{H}=1$ verschiebt.

Hiernach ergibt sich die Verbiegung von e die Biegelinie für $\mathrm{X}_{\mathrm{e}}=-1$ ist. 


\section{$-10-$}

Bestimmung der Momente. Das Mument $X_{\mathrm{e}}=-1$, wirkend in einer Ebene Stabstrecken Verbiegungen und Verdrehungen hervor. Betrachtet man die Strecke 0/1, so virkt auf diese ein Biegungsmoment

$\begin{aligned} & \mathrm{M}_{\mathrm{b}}=1 \cdot \cos \alpha_{0} \\ & \text { and ein Drehmoment }\end{aligned}$

M
$M_{9}=1 \cdot \sin \omega_{0}$.

Ersteres verbiegt den Stab-

teil $0 / 1$ in einer durch $0 / 1$

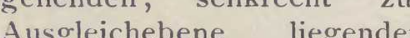

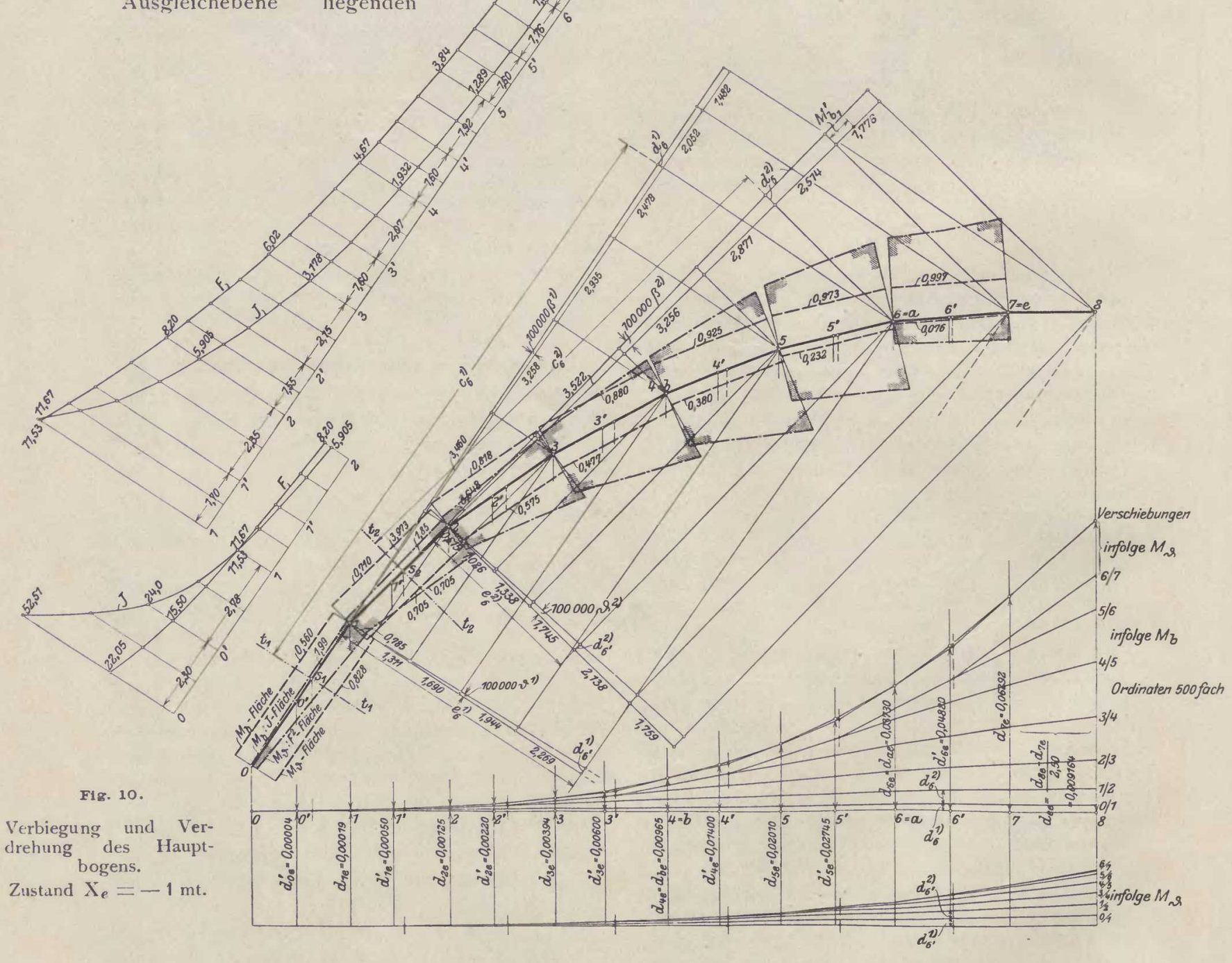

Ebene; 1

Feise

$\mathbf{M}_{\mathbf{b}}=1 \cdot \cos \kappa_{1}$,
$\mathbf{M} . r=1 \cdot \sin \kappa_{1}$ usf.
Man erhält die in Fig. 10 neben den einzelneı Bestimmung der Verbiegungen.

Man ermittelt getrennt die Verbiegungen der abn Punkte und zählt zuletzt die Exde werte zusammen. $\mathrm{Da}$ die Biegelini $\mathrm{w}=\frac{\mathrm{M}_{\mathrm{b}} \cdot \mathrm{s}}{\mathrm{J}}$ $\boldsymbol{\beta}=\frac{\mathrm{w}}{\mathrm{E}}$ und dem Polabstand E gezeichnete Seileck ist, ist alsdann die wahre Neigung der Biegelinie am Querschnitten beliebiger unregelmäßiger Form ist Ende der Belastungsfläche*). Die Werte $J$ sind hier die zulässige Annahme gemacht, daßß alle in der Ausgleichebene liegende Querschnittsachse. Verdrehungswinkel eines Stabteiles von der Längre Die Verbiegung des Stabzuges 0, 1, 2, ., 7, $8 \mathrm{er}$ - $\quad \mathrm{s}$ unter Einwirkung eines Drehmomentes M: folgt senkrecht zur Bild- oder Ausgleichebene $;=\frac{7,2}{\mathrm{~h}} \cdot \frac{\mathrm{M} ;}{\mathrm{G}} \cdot \mathrm{s}$, gemessen in Bogenmaß, worin The achse $\mathrm{t}_{1} \mathrm{t}_{1}$ senkrecht $\mathrm{zu} \quad 0 / 1$ stelt, durch de Schwerpunkt $\mathrm{S}_{1}$ der Belastungstläche geht und die sich gegen die Bildebene um den Winkel $\beta=\frac{W}{E}$ dreht. Das Maß der Verschiebung eines um $\mathrm{c}$ voin der Drehachse $t_{1} t_{1}$ entfe z. B. für Punkt 6 $\mathrm{d}_{\mathrm{m}}(1)=\mathrm{c}_{\mathrm{m}}(1) \operatorname{tg} \beta(1)$
$\mathrm{tan}^{(1)}$

$$
\mathrm{d}_{6}(1)=\mathrm{c}_{6}(1) \operatorname{tg} \beta(1)
$$$$
=\mathrm{c}_{6}{ }^{(1)} \frac{\mathrm{w}(1)}{\mathrm{E}} \text {. }
$$

= Seitenlänge des Querschnittes

$\mathrm{G}=$ Gleitmaßs ist.

Man kann setzen $\mathrm{h}^{4}=\mathrm{F}^{2} \quad$ Für das Gleitmal soll mangels ausreichender Versuchszahlen ei Mittelwert $21500000 \cdot 0385=550000$ t/ gesetzt werden. Dann erhält man

$$
y=\frac{13,1}{10^{6}} \cdot \frac{\mathrm{M}_{\mathrm{F}}, \mathrm{s}}{\mathrm{F}^{2}}=\frac{13,1}{10^{6}} \cdot \mathrm{W} \mathrm{t} / \mathrm{m}^{2}
$$

Ma in $\mathrm{tm}^{\mathrm{s}} \mathrm{s}$ in $\mathrm{m}, \mathrm{F}$ in $\mathrm{qm}$ ausgedrüclst Die Werte $\frac{\mathrm{M}_{*} \cdot}{\mathrm{F}^{2}}$ sind in Fig. 10 neben den einzelnen Die sämtlichen Verschiebungen $d_{1}(1), d_{2}(1), \ldots$ Stabstrecken aufgetragen, die einzelnen Werte bindungslinie gibt die Verbiegung des Stabzuges im Grundrißs, d. h. senkrecht zur Ausgleichebene, durch $\mathrm{M}_{\mathrm{e}}=-1$, wenn zunächst nur die Stabstrecke $0 / 1$ betrachtet wird, ein Fall, der z. B. tat sachlich eintreten lonte, wenn die Trägheit. unendlich groß wären, also diese Teile sich nich verbiegen könnten.

In gleicher Weise wird der Beitrag der Strecken $1 / 2,2 / 3$ usf. zu den Verbiegungen er-

mittelt. Es biegt $1 / 2$ um eine Achse $t_{2} t_{2}$ durch
den Schwerpunkt der Belastungsfläche $M_{\mathrm{b}}: \mathrm{J}$ um den Winkel

$$
\beta(2)=\frac{w(2)}{E}
$$

Punkt 6 z. B. verschiebt sich dann senkrecht $z$

$$
\mathrm{d}_{6}^{(2)}=\mathrm{c}_{6}^{(2)} \operatorname{tg} \beta^{(2)}=\mathrm{c}_{6}^{(2)} \frac{\mathrm{w}(2)}{\mathrm{E}} \text {. }
$$

Sämtliche Werte sind rechnerisch ermittel worden. In Fig. 10 sind die Verbiegungswinkel $\beta$ in 100000 facher Größse aufgetragen, die Verschie-
bungen werden bei einem Längenmaßstabe $1: 200$ also $\frac{100000}{200}=500$ fach

$$
\mathrm{W}=\frac{\mathrm{M} ; \mathrm{s}}{\mathrm{F}^{2}}
$$

$100000 \%=1,31 \mathrm{~W}$

sind wiederum rechnerisch ermittelt worden.

Unter alleiniger Berücksichtigung von $\mathrm{M} s$ im $2,3, \ldots, 7,8$ um die Achse 0/1, die Punkte ver-

schieben sich um die Maßse

z. B. Punkt 6 un

Die
getragen

erchebungen werden im Grundrißs autFür die nächste Stabstrecke $1 / 2$ ist it in gleicher Weise aus $\frac{\mathrm{M}_{9}}{\mathrm{~F}^{2}}$ zu ermitteln, die Verdrehung erfolot um die Achse 1/2, die Verschiebungen sind $\quad \mathrm{d}_{\mathrm{m}^{\prime}}(2)=9(2) \mathrm{e}^{(2)}$

z. B. für $6 \quad \mathrm{~d}_{6^{\prime}}(2)=g(2) \mathrm{f}_{6}^{(2)}$

Alle Verschiebungen der einzelnen Punkte zusammengezählt

In Fig. 10 sind die 100000 fachen Verdrehungen

Bestimmung der Verdrehungen.

In ähnlicher Weise wie zuvor werden auch die Verdrehungen der einzelnen Stabstrecke Mangels Un

it dem Längenmaßstab 1:200 das 500fache de wahren Werte. Diese Werte sind zum Schluß z den Verschiebungen infolge der Biegungsmoment *) Diese ist für jede Stabstrecke in 4 Teile geteilt *) Diese ist für jede Stabstrecke in 4 Teile getell
worden, was in den Figuren des kleinen Mabstabes wege
nicht dargestellt ist. Die Verdrehungen des Mittelquerschnittes e werden gemessen auf dem parallel zur
Kämpferlinie liegenden Eisatzstab $7 / 8$ und zwar 


\section{$-12-$}

als die Verschiebungen eines $1 \mathrm{~m}$ von Punkt 7 im Punkte 1

entfernten Punktes, geteilt durch $1 \mathrm{~m}$. Der Er- $\quad \mathbf{M}_{\mathrm{b}}{ }^{(1)}=1 \mathrm{p}_{6}{ }^{(1)}$

satzstab ist aus zeichnerischen Gründen länger, $\quad \mathrm{M}_{\mathrm{b}}(1)=1 \mathrm{p}_{6}$

nämlich $2,50 \mathrm{~m}$ lang gewăhlt worden. Die Ver-

drehung ist dann gegeben durch den Ordinaten-

$\mathrm{M}_{9}{ }^{(0)}=\mathrm{Mg}_{9^{(1)}}=1 q^{(0)}$

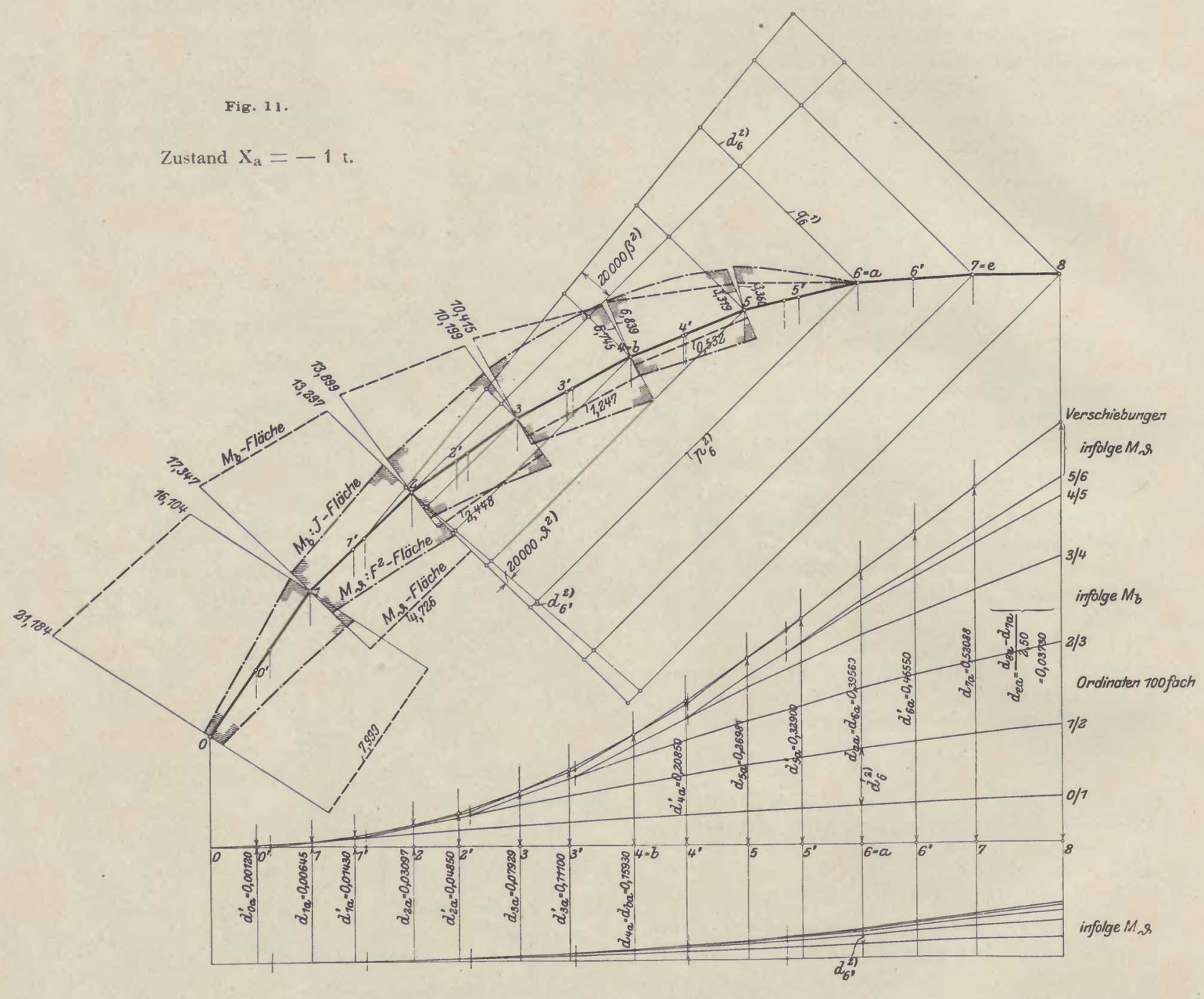

Die Verdrehung des Mittelque
die sämtlichen Lasten $\mathrm{H}$ ist nun

$$
\sum_{\mathrm{det}} \sum
$$

B) Zustand $X_{2}=-1$

Eine Einzellast $X_{2}=-1$ im Punkte $6=$ a ruft in den einzelnen Stabteilen wieder Biegungs- und

Für $0 / 1$ ist im Punkte 0 das Moment

$$
\mathrm{M}_{\mathrm{b}}{ }^{(0)}=1 \mathrm{p}_{6}^{(0)},
$$

betreffenden Querschnittsflächen, q die
von den betr. Stabachsen sind (Fig. 11).

Die Werte $\mathrm{p}$ und $\mathrm{q}$ sind rechnerisch bestimmt

Tan bildet wieder tür die Verbiegungen die Werte $\frac{M_{b}}{J}$, sucht den Schwerpunkt der w-Fläche und ermittelt den Verbiegungswinkel

$$
\beta=\frac{\mathrm{w}}{\mathrm{E}}
$$

die Verschiebungen sind wieder $\mathrm{d}_{\mathrm{m}}=\mathrm{c}_{\mathrm{m}} \cdot \frac{\mathrm{w}}{\mathrm{E}}$. Für die Verdrehungen bildet man wieder $\frac{\mathrm{M}_{s},}{\mathrm{~F}^{2}}, s=\frac{13,1}{10^{6}} \mathrm{~W}, \mathrm{~d}_{\mathrm{m}}=\mathrm{e}_{\mathrm{m}} \vartheta$. Aufgetragen wurden die $\mathrm{Nei}$ gungen $\boldsymbol{\beta}$ und $\%$ in 200 ocofacher Gröłe, die Verschiebungen sind also

$20000=100$ fach dargestell worden.

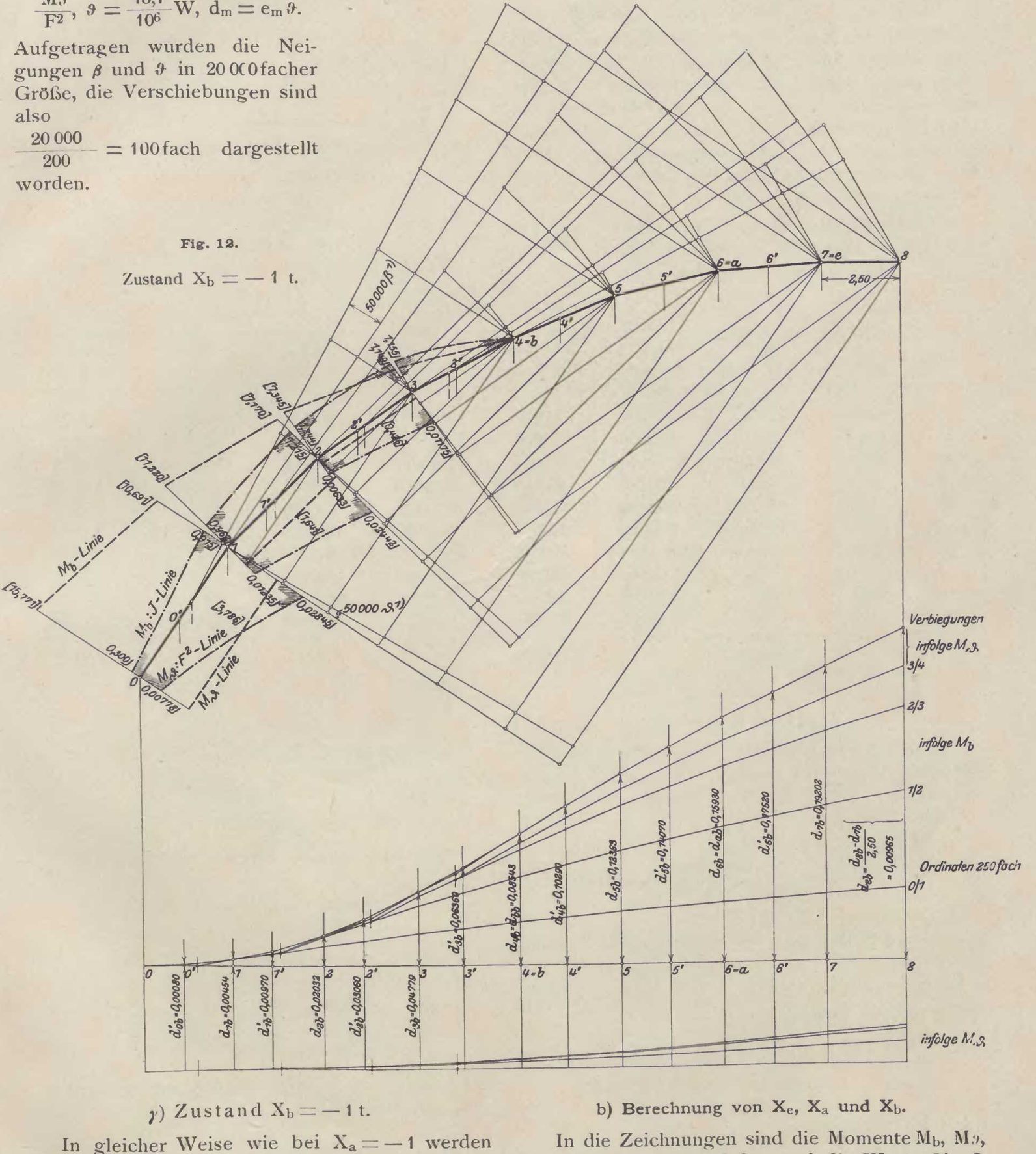

b) Berechnung von $\mathrm{X}_{\mathrm{e}}, \mathrm{X}_{\mathrm{a}}$ und $\mathrm{X}_{\mathrm{b}}$

der Maßstab für die Winkeländerungen 50000 , die Verschieburgen sind also in $50000=250$ facter Größe aufgetragen (Fig. 12). 2000 facher In gleicher Weise wie bei $x_{a}=-1$ werden In die Zeichnungen sind die Momente $M_{b}, M_{:}$:
a die Verschiebungen für die Einzellast $\mathrm{X}_{\mathrm{b}}=-1 \mathrm{t} \quad \mathrm{M}_{3}: \mathrm{F}^{2}$ eingetragen. Sämtliche Zahlen sind beim Punkt $4=$ b ermittelt. In der Zeichnung ist rechnete Werte 
$14-$

Als Probe für die Richtigkeit der Verschie-

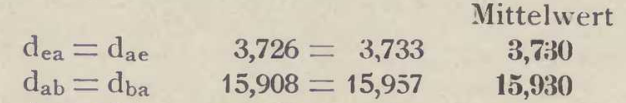

$\begin{array}{lll}\mathrm{d}_{\mathrm{ab}}=\mathrm{d}_{\mathrm{ba}} & 15,908=15,957 & \mathbf{1 5 ,}, 902 \\ \mathrm{~d}_{\mathrm{be}}=\mathrm{d}_{\mathrm{eb}} & 0,9688=0,9618 & \mathbf{0 , 9 6 5 0}\end{array}$

Es ist somit sehr gute Übereinstimmung er-

zielt worden. Die aus gebrochenen Linienzügen bestehenden Biegelinien sind zwischen den einwiseglichen worden, aus der die Ortige Kurve, $d_{\mathrm{ma}} \mathrm{d}_{\mathrm{mb}}$ an den Lastzwischenpunkten $0^{\prime}, 1^{\prime}, 2^{\prime}$ $3^{\prime}, \ldots, 6^{\prime}$ für die Berechnung der Kräfte $\mathrm{X}_{\mathrm{e}}, \mathrm{X}_{\mathrm{a}}$ und

Mit den Krăften $\mathrm{H}_{\mathrm{m}}=0,55 \mathrm{P}_{\mathrm{m}}$ erhält man die $\mathrm{H}_{m} \mathrm{~d}_{m e} \mathrm{H}_{m}$ d

\begin{tabular}{|c|c|c|c|c|c|c|c|c|}
\hline Punkt & $\begin{array}{r}P_{m} \\
\text { in } t\end{array}$ & $\begin{array}{l}\mathrm{H}_{\mathrm{m}} \\
\text { in } \mathrm{t}\end{array}$ & $\begin{array}{c}\mathrm{d}_{\mathrm{me}} \\
\text { in } \mathrm{mm}\end{array}$ & $\begin{array}{l}\mathrm{H}_{\mathrm{m}} \mathrm{d}_{\mathrm{m}} \\
\text { in tmm }\end{array}$ & $\begin{array}{c}\mathrm{d}_{\mathrm{ma}} \\
\text { in } \mathrm{mm}\end{array}$ & $\begin{array}{l}\mathrm{H}_{\mathrm{m}} \mathrm{d}_{\mathrm{ma}} \\
\text { in tmm }\end{array}$ & $\begin{array}{c}\mathrm{d}_{\mathrm{mb}} \\
\text { in } \mathrm{mm}\end{array}$ & $\begin{array}{l}\mathrm{H}_{\mathrm{m}} \mathrm{d}_{\mathrm{mb}} \\
\text { in } \mathrm{tmm}\end{array}$ \\
\hline $0^{\prime}$ & 232,0 & 128,0 & 0,00004 & 0,0051 & 0,00120 & 0,1536 & 0,00080 & 0,1024 \\
\hline $1^{\prime}$ & 105,4 & 58,0 & 0,00050 & 0,0290 & 0,01480 & 0,8584 & 0,00870 & 0,5626 \\
\hline 2 & 235,1 & 129,7 & 0,00125 & 0,1621 & 0,03097 & 4,0168 & 0,02032 & $2,63: 5$ \\
\hline$z^{\prime}$ & 69,8 & 38,4 & 0,00220 & 0,0845 & $0,0+850$ & 1,8624 & 0,03060 & 1,1750 \\
\hline $3^{\prime}$ & 50,7 & 27,9 & 0,00600 & 0.1674 & 0,11100 & 3.0969 & 0,06360 & 1,7744 \\
\hline $4=b$ & 201,4 & 111,0 & & & & 17,6823 & 0,08543 & 9,4827 \\
\hline $\begin{array}{l}4^{\prime} \\
5^{\prime}\end{array}$ & $\begin{array}{l}55,2 \\
0.4\end{array}$ & 30,4 & 0,01400 & 0,4256 & 0,20850 & 6,3384 & 0,10290 & 3,1282 \\
\hline 5 & 35,6 & 19,6 & 0,02745 & 0,5380 & 0,32900 & 6,4484 & 0,14070 & 2,7577 \\
\hline $\begin{array}{l}6=a \\
6^{\prime}\end{array}$ & $\begin{array}{r}190,0 \\
25,2\end{array}$ & $\begin{array}{c}100,1 \\
13,8\end{array}$ & $\begin{array}{l}0,03730 \\
0,04800\end{array}$ & $\begin{array}{l}3,7337 \\
0.6734\end{array}$ & $\begin{array}{l}0,39569 \\
0,45550\end{array}$ & $\begin{array}{r}39,60866 \\
6,269\end{array}$ & $\begin{array}{l}0,15930 \\
0,01750\end{array}$ & $\begin{array}{r}5,9459 \\
2,478\end{array}$ \\
\hline & & Summe & & 6,8900 & & 86,3517 & & 39,9822 \\
\hline
\end{tabular}

(c) Hauptnetz und $X$.

Es soll zuerst die Kraft $X_{e}$ ermittelt werden die unter Einwirkung der Kräfte $\mathrm{H}_{\mathrm{m}}$ auftritt, wenn $\mathrm{X}_{\mathrm{a}}$ und $\mathrm{X}_{\mathrm{b}}=0$ sind, die Strebenbinder also fehlen.

$\sum \mathrm{H}_{\mathrm{m}} \mathrm{d}_{\mathrm{me}}$

dee

$6,8900 \mathrm{tmm}$

Es würde also ein Moment von rd. $752 \mathrm{tm}$ im Unter der Finwirkung dieses Nomentes und der 1-Krafte ergeben sich die Verschiebungen a unkte a und b 2

a) $\sum \mathrm{H}_{\mathrm{m}} d_{\mathrm{ma}}=\sum \mathrm{H}_{\mathrm{m} \mathrm{d}_{\mathrm{ma}}-\mathrm{X}_{\mathrm{e}} \mathrm{d}_{\mathrm{ae}}}$ $=86,3517-751,86 \cdot 0,03730$

b) $\sum_{\mathrm{H}_{\mathrm{m}} \boldsymbol{\Delta}_{\mathrm{mb}}}=\sum \mathrm{H}_{\mathrm{m}} \mathrm{d}_{\mathrm{mb}}-\mathrm{X}_{\mathrm{e}} \mathrm{d}_{\mathrm{be}}$ $=39,9822-751,86 \cdot 0,0096$

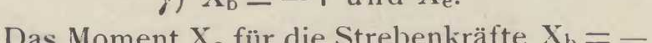
$\mathrm{X}_{\mathrm{e}}=\frac{\mathrm{d}_{\mathrm{be}}}{\mathrm{d}_{\mathrm{ee}}}=\frac{0,009650}{0,009,164}=1,0530 \mathrm{tm}$.

Die Verschiebungen von a und b betrasen: a) $\begin{aligned} \boldsymbol{t}_{\mathrm{ab}} & =\mathrm{d}_{\mathrm{ab}}-\mathrm{X}_{\mathrm{e}} \cdot \mathrm{d}_{\mathrm{ae}} \\ & =0,15930-1,0530 \cdot 0,03730\end{aligned}$ $=0,1200 \mathrm{~mm}$

in Übereinstimmung mit dem unter $X_{a}=-1$ und

$=\mathrm{d}_{\mathrm{bb}}-X_{\mathrm{e}} \cdot \mathrm{d}_{\mathrm{be}}$
$=0,08543-1,0530 \cdot 0,00965$
$=0,0753$

1) Verschiebungen der Strebenbinder durch die Eigenlas $X$-Kräfte.

In das System sind jetzt nocli die Verschiebungen der Strebenbinder einzuführen und zwar
$-15-$

Hauptnetz sowvie für die Zustände $X_{a}=-1$ un

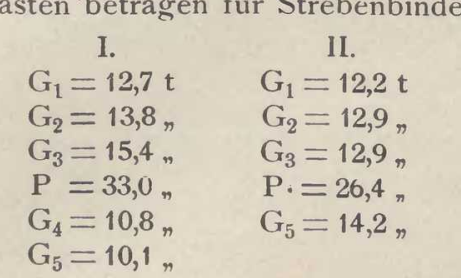

Mit Hilfe der w-Kräfte sind in bekannter Weis 列 suchung für den Strebenbinder II wiedergeomeben.

作 der Kräfte $G$ und $P$ die in folgender Ubèrsicht aufgefuhrten Verschiebungen und mit den Laste $P_{m}$ die Werte $P_{m} s_{m a}$, welches die Verschiebunge Kämpferlinie sind.
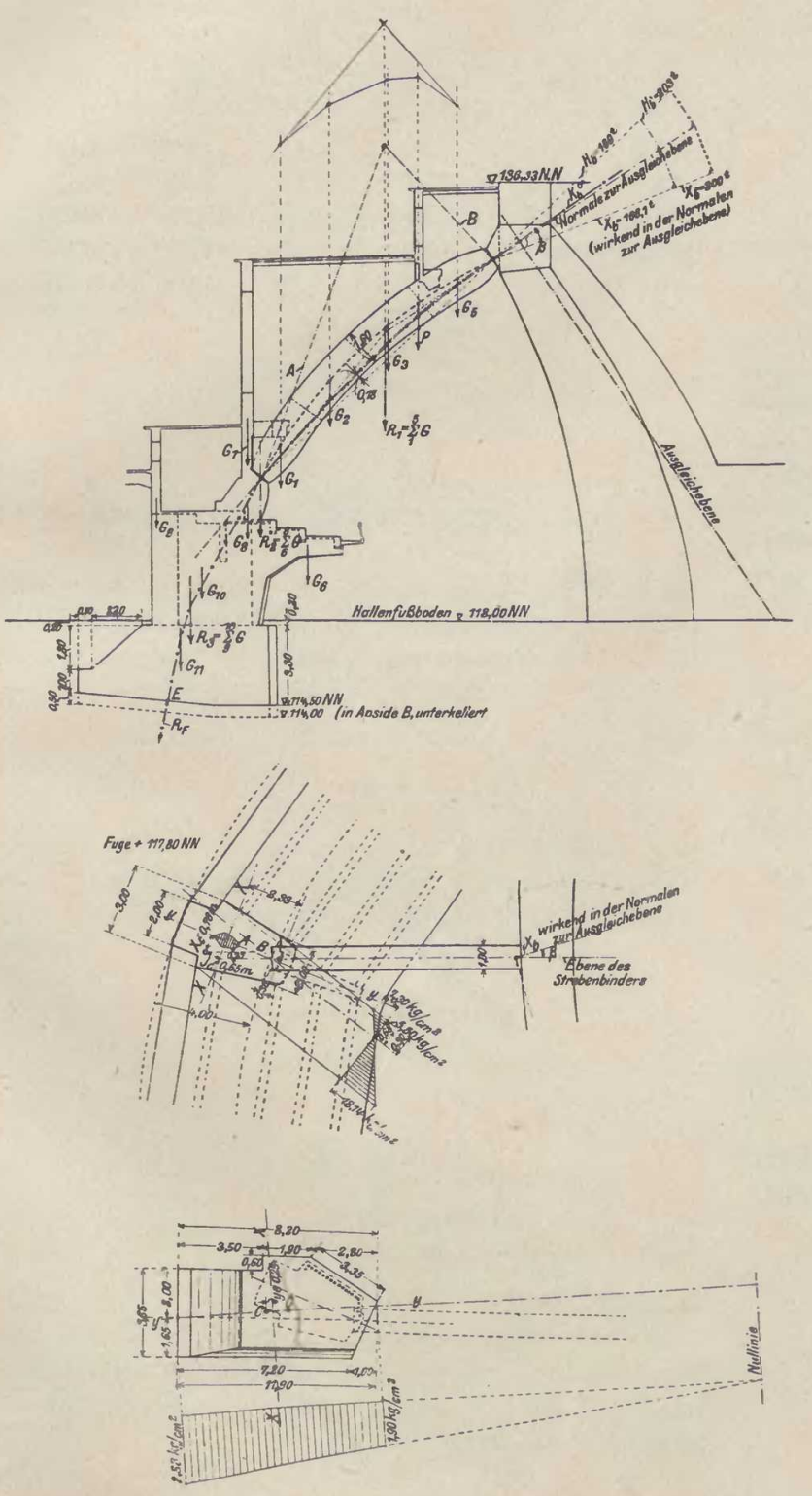

Fig. 13.

Untersuchung des Strebenbinders I

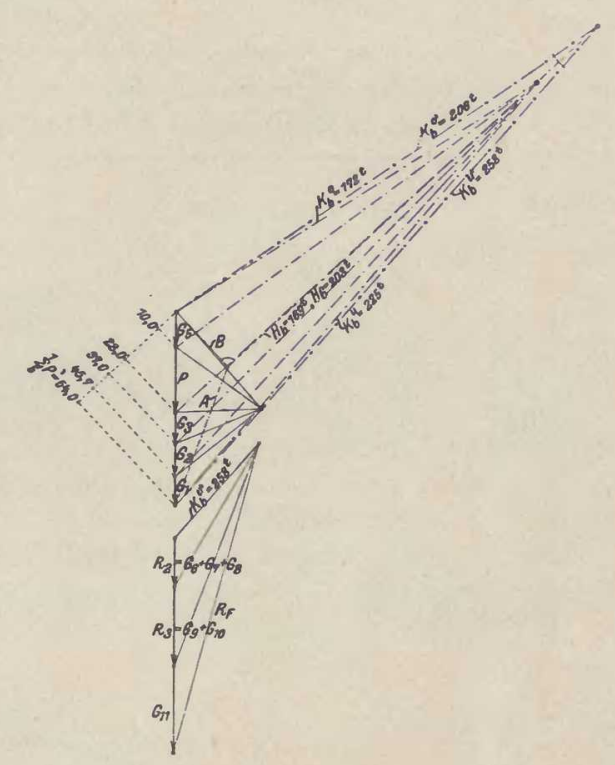

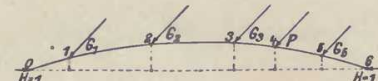

Hithith

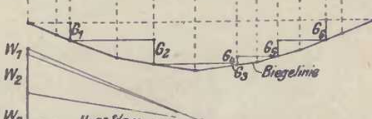

wi:

强和 
$-16-$

\begin{tabular}{|c|c|c|c|}
\hline \multicolumn{4}{|c|}{ Strebenbinder I. } \\
\hline Punkt & $\delta_{\mathrm{ma}}$ & $\mathrm{P}_{\mathrm{m}}$ & $\mathrm{P}_{\mathrm{m}} J_{\mathrm{ma}}$ \\
\hline & $+0,0141$ & 12,7 & $+0,179 \mathrm{~mm}$ \\
\hline 2 & $+0,0393$ & 13,8 & $+0,542$ \\
\hline 3 & $\begin{array}{r}+0,0480 \\
+0\end{array}$ & 48,8 & $+2,340$ \\
\hline \multirow{3}{*}{$\begin{array}{l}4 \\
5\end{array}$} & $+0,0300$ & 10,8 & $+0,324$, \\
\hline & $-0,0030$ & 10,1 & $-0,030$ \\
\hline & & & $+3,355 \mathrm{~mm}$ \\
\hline
\end{tabular}

\begin{tabular}{|c|c|c|c|}
\hline \multicolumn{4}{|c|}{ Strebenbinder II. } \\
\hline Punkt & $J_{\mathrm{mb}}$ & $P_{m}$ & $P_{m} \delta_{m b}$ \\
\hline 1 & $+0,0090$ & 12,2 & $+0,110 \mathrm{~mm}$ \\
\hline 2 & $+0,0237$ & 12,9 & $+0,306$ \\
\hline 3 & $+0,0252$ & 12,9 & $+0,325$ \\
\hline 4 & $\begin{array}{l}+0,0182 \\
+0,0034\end{array}$ & $\begin{array}{l}26,6 \\
14.2\end{array}$ & $\begin{array}{l}+0,484 \quad n \\
+0,048\end{array}$ \\
\hline & & 14,2 & $+0,060 \mathrm{n}$ \\
\hline
\end{tabular}
Die Verschiebungen der oberen Kämpfer-
punkte durch $\mathrm{H}=-1$ betragen $j_{\mathrm{az}}=0,0330 \mathrm{~mm}$

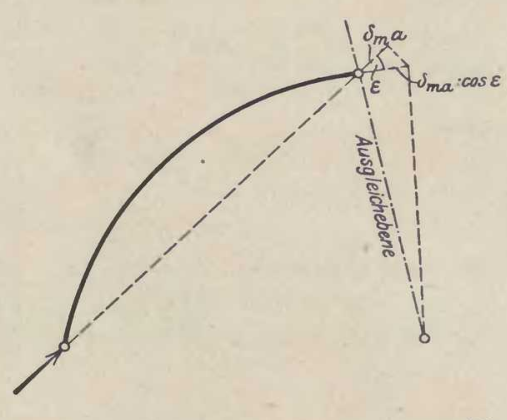

F18 14

und $\jmath_{\mathrm{bb}}=0,0200 \mathrm{~mm}$, die (in der Rechnung nicht weiter auftretenden) aktiven Bogenschübe würden

$$
\mathrm{H}_{\mathrm{a}}=\frac{\sum_{\mathrm{J}_{\mathrm{aa}}} \mathrm{P}_{\mathrm{ma}}}{v_{\mathrm{a}}}=\frac{3,355}{0,0330}=101,7 \mathrm{t},
$$$$
H_{b}=\frac{1,273}{0,0200}=63,7 \mathrm{t} .
$$

sichtigen. Die Verkürzung der Stabachse beträgt $\mathfrak{s}=\sum \frac{\mathrm{P}^{\prime} \mathrm{s}}{\mathrm{EF}}$, worin $\mathrm{P}^{\prime}$ die Projektion der Lasten auf die Stabachse oder, genau genug, auf die mpferlinie ist.

Man erhält für die Lasten $\mathrm{G}$ und $\mathrm{P}$ folgende

\begin{tabular}{|c|c|c|c|c|c|}
\hline \multicolumn{3}{|c|}{ Binder I. } & \multicolumn{3}{|c|}{ Binder II. } \\
\hline $\mathrm{P}^{\prime}$ & s & $\mathrm{P}^{\prime} \mathrm{s}$ & $\mathrm{P}^{\prime}$ & $\mathrm{s}$ & $P^{\prime} s$ \\
\hline $\mathrm{t}$ & $\mathrm{m}$ & $\mathrm{tm}$ & $\mathrm{t}$ & $\mathrm{m}$ & $\mathrm{tm}$ \\
\hline 64,0 & 1,75 & 112,0 & 54,0 & 1,70 & 91,8 \\
\hline 55,4 & 3,55 & 196,7 & 45,7 & 3.35 & 153,1 \\
\hline 46,2 & 3,85 & 177,9 & 37,0 & 3,35 & 124,0 \\
\hline \multirow{3}{*}{6,8} & 3,85 & 46,2 & 28,0 & 1,70 & 47,6 \\
\hline & 2,75 & 18,7 & 10,0 & 1,95 & 19,5 \\
\hline & & 551,5 & & & 436,0 \\
\hline
\end{tabular}

\section{Binder I.}

$y=\frac{55150000 \mathrm{cmkg}}{140000 \mathrm{~kg} / \mathrm{qcm} \cdot 16000 \mathrm{qcm}}=0,0246 \mathrm{~cm}$ $0,246 \mathrm{~mm}$.

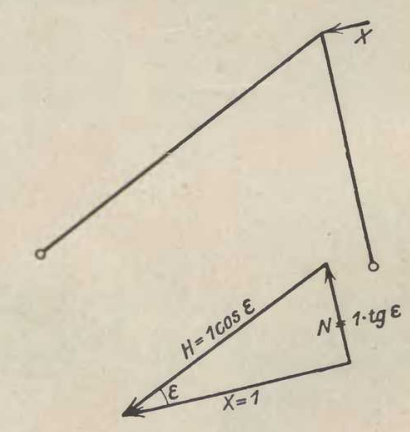

$J=\frac{43600000 \mathrm{cmkg}}{140000 \mathrm{~kg} / \mathrm{qcm} \cdot 16000 \mathrm{~cm}}=0,0195 \mathrm{~cm}$ $=0,195 \mathrm{~mm}$.

Die Verschiebung in der Kämpferlinie unter den

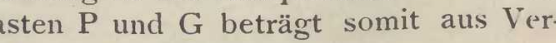

$$
\text { Binder I. }
$$

Es ist noch der Einfluf der Verkürzung der
Strebenbinder durch die Normalkräfte zu berück$v_{\text {ma }}=3,357-0,246=3,111 \mathrm{~mm}$
Binder II.

in $=1,273-0,195=1,078 \mathrm{~mm}$

In gleicher Weise ist die Verkürzung für $\mathrm{H}=1$$$
\text { Binder I. }
$$

$$
J=\frac{1000 \mathrm{~kg} \cdot 1650 \mathrm{~cm}}{140000 \mathrm{~kg} / \mathrm{qcm} \cdot 16000}
$$
$=0,00737 \mathrm{~m}$.

$$
\text { Binder II. }
$$

$J=\frac{1000 \mathrm{~kg} \cdot 1350 \mathrm{~cm}}{140000 \mathrm{~kg} / \mathrm{qcm} \cdot 16000 \mathrm{~cm}}=0,000603 \mathrm{~cm}$ $=0,00603 \mathrm{~mm}$.

Insgesamt ist die Verschiebung (Verkürzung der tabachse durch Biegungsmoment und durch N

$$
\text { Binder I. }
$$

$J_{\mathrm{aa}}=0,03300+0,00737=0,04037 \mathrm{~mm}$.

$$
\text { Binder II. }
$$

$\mathrm{bb}=0,02000+0,00603=0,02603 \mathrm{~mm}$

Ermittelt waren die Verschiebungen der Strebe 列 ene; sie betragen in dieser Richtung

$$
v^{\prime}=\frac{\delta}{\cos \varepsilon},
$$

worin $\varepsilon=$ Winkel $z$ wischen der Kämpferlinie und der Normalen zur Ausgleichebene ist. Es sin

$$
\begin{gathered}
J_{\mathrm{ma}}: \cos \mathrm{ta}_{\mathrm{a}} \text { und } J_{\mathrm{mb}}: \cos \mathrm{e}_{\mathrm{b}}, \\
J_{\mathrm{ma}}^{\prime}=3,111: 0,988=3,1488 \mathrm{~mm}
\end{gathered}
$$

und

$$
\Delta_{\mathrm{mb}}^{\prime}=1,078: 0,984=1,0955 \mathrm{~mm} .
$$

Einer Kraft $X=1$ entspricht $X=\frac{H}{\cos \varepsilon}$; diese Kraft ergibt eine Verschiebung in der Richtung senkrecht zur Ausgleichebene sind einzuführen:

$$
\begin{gathered}
J_{\mathrm{aa}}: \cos ^{2} \mathrm{ta}_{\mathrm{a}} \text { und } \delta_{\mathrm{bb}}: \cos ^{2} \mathrm{e}_{\mathrm{b}} \text {, } \\
\boldsymbol{J}_{\mathrm{aa}}=0,0414 \mathrm{~mm},
\end{gathered}
$$$$
\Delta^{\prime} \mathrm{bb}=0,0269 \mathrm{~mm}
$$

c) Zusammenstellung.

Man erhält mit den vorstehend ermittelten Ve Strebenbinder die folgende

Eigengèwicht.

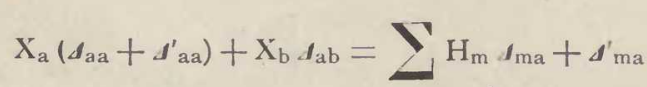
$\mathrm{X}_{\mathrm{a}} d_{\mathrm{ab}}+\mathrm{X}_{\mathrm{b}}\left(\Delta_{\mathrm{bb}}+\boldsymbol{s}_{\mathrm{bb}}\right)=\sum \mathrm{H}_{\mathrm{m}} d_{\mathrm{mb}}+\Delta_{\mathrm{mb}}^{\prime}$

$\mathrm{X}_{\mathrm{a}} \cdot 0,2853+\mathrm{X}_{\mathrm{b}} \cdot 0,1200=61,4561$
$\mathrm{X}_{\mathrm{a}} \cdot 0,1200+\mathrm{X}_{\mathrm{b}} \cdot 0,1022=33,023$ $\mathrm{X}_{\mathrm{a}} \cdot 0,1200+\mathrm{X}_{\mathrm{b}} \cdot 0,1022=33,8223$

$\mathrm{X}_{\mathrm{a}}=150,568 \mathrm{t}$,

$\mathrm{X}_{\mathrm{b}}=154,158 \mathrm{t}$

Das im System wirkende Moment $\mathrm{X}_{\mathrm{e}}$ setz sirkung der H-Kraffte, nämlich $X_{0}=751,06$ in ferner dem im System $X_{a}=150,568$ und $X_{b}=154,158$ wirkenden, wobel fur $\mathrm{X}_{\mathrm{a}}=-1$ das Moment $\mathrm{X}_{e}=$ $4,0703 \mathrm{mt}$, für $\mathrm{X}_{\mathrm{b}}=-1$ das Moment $\mathrm{X}_{\mathrm{e}}=1,0530 \mathrm{~m}$

$X_{e}=751,86-150,568 \cdot 4,0703-154,158 \cdot 1,0530$

$$
\mathrm{X}_{\mathrm{e}}=-\mathbf{2 3 , 3 2 5 \mathrm { s }} \mathrm{mt} \text {. }
$$$$
\text { Wärmeänderung. }
$$

Für Wärmeänderung um $\pm 7,5^{\circ}$ ändert sich die Strebenlänge um

$$
\Delta_{1}=\frac{1 \cdot 7,5}{80000},
$$

d. i. bei a) um $\boldsymbol{d}_{1}=1,5469 \mathrm{~mm}$,

$$
\text { "b) " } A_{1}=1,2656 \text {, }
$$

Da die Wärmeänderung des Hauptbogens sich in senkrecht zu der Strebenkämpferlinie steht, verschwindet dieser Einfluß nahezu völlig. Man erhält die beiden Gleichungen:

$\mathrm{X}_{\mathrm{at}} \cdot 0,2653+\mathrm{X}_{\mathrm{bt}} \cdot 0,1200=1,5469$, $\mathrm{X}_{\mathrm{ar}} \cdot 0,1200+\mathrm{X}_{\mathrm{bt}} \cdot 0,1022=1,2656$

$$
\mathrm{X}_{\mathrm{at}}=0,423 \mathrm{t} \text {, }
$$$$
X_{b t}=11,88 \bar{t} t \text {. }
$$

$\mathrm{X}_{\mathrm{et}}=-4,0703 \cdot \mathrm{X}_{\mathrm{at}}-1,0530 \cdot \mathrm{X}_{\mathrm{bt}}=-4,0703 \cdot 0,423$ $-1,0530 \cdot 11,887=-\frac{14.239}{2} \mathrm{mt}$ 
$-18-$ $\begin{array}{ll}\text { Nachgiebigkeit der Streben pfeiler. } & \text { vergrößert, diese Vergrößerung wurde dann als } \\ \text { Es soll noch der Einfluß einer ungleichen Sen- } & \text { gleichbleibend für sämtliche Querschnitte ange- }\end{array}$ kung der Strebenbinder ermittelt werden und zwar nommen. Für die Streben wurde mit der tatsächfür ein $d_{\mathrm{a}}=-1 \mathrm{~mm}$ und ein $\Delta_{\mathrm{b}}=-1$, je getrennt lichen Bewehrung von $0,8 \%$ gerechnet, womit das (1) Man erWerte, wobei die Kärmewirkung $\mathrm{x}_{\mathrm{a}}=-6,925 \mathrm{t}$

in gleicher Weise ist für $\iota_{\mathrm{b}}=-1 \mathrm{~mm}$, $\mathrm{X}_{\mathrm{a}}=+8,132 \mathrm{t}$,
$\mathrm{X}_{\mathrm{b}}=-19,334 \mathrm{t}$ die Zahlen ohne Berüchsichtigung der Eiseneinagen darstellen $\begin{array}{lll}\mathrm{X}_{\mathrm{a}} & 152,0 \mathrm{t}(150,568) \\ \mathrm{X}_{\mathrm{b}} & 15,5 \mathrm{t}(154,158) \\ \mathrm{X}_{\mathrm{e}} & -32 \mathrm{mt}(-23,325) \\ \end{array}$ Es tritt in den gefährlichen Querschnitten in
der Nähe des Kämpfers eine geringe Entlastung Senken sich zugleich die Pfeiler der beim ein, wenn die Eisenbewehrung berücksichtigt (d. h. in lotrechter Richtung etwa 1,5 $\mathrm{mm}$ ), so ad- wird, es ist also zu ungünstig gerechnet, wenn sie vernachlassigt worden ist. Bbenso witt in $\mathrm{X}_{\mathrm{a}}=+1,2 \mathrm{t}, \quad \mathrm{X}_{\mathrm{b}}=-11,2 \mathrm{t}$.

5) Berücksichtigung der durch die Eisen c) Ermittlung der Biegungsmomente senkrecht zur inlagen vergroßsten Trägheitsmomente. Die Untersuchung ist noch durchgeführt wor-

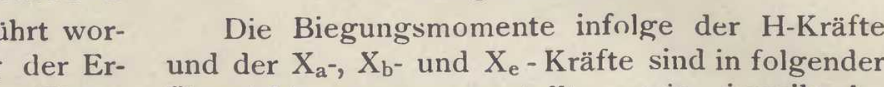
Übersicht zusammengestellt, worin jeweils $h$ bogen wurde eine die senkrechten Abstände der Kräfte H $\begin{array}{lll}\text { solche von } 0,4 \% \text { eingeführt, die das Trägheits- } & \text { von den } \\ \text { moment in einem mittleren Querschnitte um } 8 \% & 2, \ldots \text { sind }\end{array}$

Biegungsmomente senkrecht zur Ausgleichebene.

\begin{tabular}{|c|c|c|c|c|c|c|c|c|c|c|c|c|c|c|c|c|c|}
\hline & & & & & & & & & & & & & & & & & \\
\hline \multirow{2}{*}{ Punkt } & \multirow{2}{*}{$\begin{array}{l}\text { Kraft } \\
\mathrm{H} \text { in } \mathrm{t}\end{array}$} & \multicolumn{2}{|c|}{0} & \multicolumn{2}{|c|}{1} & \multicolumn{2}{|c|}{2} & \multicolumn{2}{|c|}{3} & \multicolumn{2}{|c|}{4} & \multicolumn{2}{|r|}{5} & \multicolumn{2}{|c|}{6} & \multicolumn{2}{|c|}{7} \\
\hline & & $\mathrm{h}_{\mathrm{m}}$ & $\mathrm{H}_{\mathrm{m}} \mathrm{h}_{\mathrm{m}}$ & $\mathrm{h}_{\mathrm{m}}$ & $\mathrm{H}_{\mathrm{m}} \mathrm{h}_{\mathrm{m}}$ & $\mathrm{h}_{\mathrm{m}}$ & $\mathrm{H}_{\mathrm{m}} \mathrm{h}_{\mathrm{m}}$ & $\mathrm{h}_{\mathrm{m}}$ & $\mid \mathrm{H}_{\mathrm{m}} \mathrm{h}_{\mathrm{m}}$ & $\mathrm{h}_{\mathrm{m}}$ & $\mathrm{H}_{\mathrm{m}} \mathrm{h}_{\mathrm{m}}$ & $\mathrm{h}_{\mathrm{m}}$ & $\mathrm{H}_{\mathrm{m}} \mathrm{h}_{\mathrm{m}}$ & $\mathrm{h}_{\mathrm{m}}$ & $\mathrm{H}_{\mathrm{m}} \mathrm{h}_{\mathrm{m}}$ & $\mathrm{h}_{\mathrm{m}}$ & $\mathrm{H}_{\mathrm{m}} \mathrm{h}_{\mathrm{m}}$ \\
\hline $0^{\prime}$ & $\begin{array}{l}128,0 \\
5 \circ 0\end{array}$ & $\begin{array}{r}2,30 \\
675\end{array}$ & & & & & & & & & & & & & & & \\
\hline $\begin{array}{l}1^{\prime} \\
2\end{array}$ & & 6 & & $\begin{array}{l}1,70 \\
4,05\end{array}$ & \begin{tabular}{|l}
099 \\
525
\end{tabular} & & & & & & & & & & & & \\
\hline $2^{\prime}$ & & & & & 214 & 1,55 & 60 & & & & & & & & & & \\
\hline 3 & & & & & & 5,29 & 148 & 1,60 & & & & & & & & & \\
\hline 4 & 111,0 & 15, & 1750 & 11,22 & 1245 & 7,35 & 816 & $\begin{array}{l}3,67 \\
50\end{array}$ & $\begin{array}{l}407 \\
160\end{array}$ & & & & & & & & \\
\hline $\begin{array}{l}4 \\
5\end{array}$ & $\begin{array}{l}30,4 \\
196,\end{array}$ & $\begin{array}{l}17, \\
19\end{array}$ & $\begin{array}{l}520 \\
390\end{array}$ & $\begin{array}{l}12,70 \\
15,85\end{array}$ & $\begin{array}{l}386 \\
311\end{array}$ & $\begin{array}{r}8,91 \\
12,26\end{array}$ & $\begin{array}{l}2711 \\
240\end{array}$ & $\begin{array}{l}5,26 \\
8,72\end{array}$ & $\begin{array}{l}600 \\
171\end{array}$ & $\begin{array}{l}1,60 \\
5,10\end{array}$ & $\begin{array}{r}49 \\
100\end{array}$ & & & & & & \\
\hline $\begin{array}{l}5 \\
6\end{array}$ & $\begin{array}{r}19,6 \\
100,1\end{array}$ & $\begin{array}{l}19,89 \\
2118\end{array}$ & $\begin{array}{r}390 \\
2120\end{array}$ & $\begin{array}{l}15,85 \\
17,35\end{array}$ & \begin{tabular}{|r}
311 \\
1737
\end{tabular} & $\begin{array}{l}12,26 \\
13.90\end{array}$ & $\begin{array}{r}240 \\
1391\end{array}$ & $\begin{array}{r}8,72 \\
10,42\end{array}$ & $\begin{array}{c}171 \\
1043\end{array}$ & $\begin{array}{l}, 110 \\
6,84\end{array}$ & $\begin{array}{l}100 \\
685\end{array}$ & $\begin{array}{l},, 00 \\
3,36\end{array}$ & 336 & & & & \\
\hline $6^{\prime}$ & $\begin{array}{r}13,8 \\
\end{array}$ & $\begin{array}{l}2,1,18 \\
22,17\end{array}$ & 306 & $\begin{array}{l}1,30 \\
18,57\end{array}$ & $\begin{array}{r}737 \\
256\end{array}$ & $\begin{array}{l}13,90 \\
15,27\end{array}$ & $\begin{array}{r}231 \\
211\end{array}$ & $\begin{array}{l}0,42 \\
11,88\end{array}$ & \begin{tabular}{|c|c|c|}
8 & 164 \\
\end{tabular} & $\begin{array}{l}8,044 \\
8,36\end{array}$ & $\begin{array}{l}115 \\
115\end{array}$ & $\begin{array}{l}4,94 \\
4,50\end{array}$ & 68 & 1,60 & 22 & & \\
\hline \multicolumn{2}{|c|}{$\Sigma \mathrm{a}=$} & \multicolumn{2}{|c|}{-7738} & \multicolumn{2}{|c|}{-5031} & \multicolumn{2}{|c|}{-3137} & \multicolumn{2}{|r|}{-1990} & \multicolumn{2}{|r|}{-949} & \multicolumn{2}{|r|}{-435} & \multicolumn{2}{|r|}{-22} & & \\
\hline \multirow{2}{*}{\multicolumn{2}{|c|}{$\begin{array}{l}\mathrm{X}_{\mathrm{a}}=150,6 \\
\mathrm{X}_{\mathrm{b}}=154,2\end{array}$}} & 21,18 & 33190 & 17,35 & 2613 & 13,90 & 2093 & $\begin{array}{l}10,42 \\
\end{array}$ & $\begin{array}{ll}21569 \\
2\end{array}$ & 6,84 & 1030 & 3,36 & 506 & & & & \\
\hline & & 15,77 & 2432 & 11,22 & 1730 & 7,35 & 1133 & 3,67 & \begin{tabular}{l|l}
7 & 566
\end{tabular} & & & & & & & & \\
\hline \multicolumn{2}{|c|}{$\mathrm{x}_{\mathrm{e}}=-23,3$} & $0,560^{*}$ & * $-13,0$ & 0,710 & $-16,5$ & 0,818 & $-19,1$ & 0,880 & $0-20,5$ & 0,925 & $-21,6$ & \begin{tabular}{|l|l}
0,973 \\
\end{tabular} & $3-22,7$ & \begin{tabular}{|l|l|}
0,997 \\
\end{tabular} & $-23,2$ & 1,000 & $0-23$, \\
\hline \multicolumn{2}{|c|}{$\Sigma_{b}=$} & \multicolumn{2}{|c|}{+5609} & \multicolumn{2}{|c|}{+4326} & \multicolumn{2}{|c|}{+3207} & \multicolumn{2}{|c|}{+2114} & \multicolumn{2}{|c|}{+1008} & \multicolumn{2}{|r|}{+483} & \multicolumn{2}{|c|}{$-23,2$} & \multicolumn{2}{|r|}{-23} \\
\hline \multicolumn{2}{|c|}{$\Sigma_{a}-\Sigma_{b}=$} & & -2129 & & -705 & & $+\quad 70$ & & +124 & & $\begin{array}{l}+\quad 59 \\
\end{array}$ & & +48 & & -45 & & -23 \\
\hline
\end{tabular}

\begin{tabular}{|c|c|c|c|c|c|c|c|c|c|c|c|c|c|c|c|c|}
\hline & \multicolumn{2}{|r|}{0} & \multicolumn{2}{|r|}{1} & \multicolumn{2}{|r|}{2} & \multicolumn{2}{|c|}{3} & \multicolumn{2}{|c|}{4} & \multicolumn{2}{|r|}{5} & \multicolumn{2}{|r|}{6} & \multicolumn{2}{|r|}{7} \\
\hline & $\mathrm{h}_{\mathrm{m}}$ & $\mathrm{H}_{\mathrm{m}} \mathrm{h}_{\mathrm{m}}$ & $\mathrm{h}_{\mathrm{m}}$ & $\mathrm{H}_{\mathrm{m}} \mathrm{h}_{\mathrm{m}}$ & $h_{m}$ & $\left|\mathrm{H}_{\mathrm{m}} \mathrm{h}_{\mathrm{m}}\right|$ & $h_{m}$ & $\mathrm{H}_{\mathrm{m}} \mathrm{h}_{\mathrm{m}}$ & $h_{m}$ & $\mathrm{H}_{\mathrm{m}} \mathrm{h}_{\mathrm{m}}$ & $h_{m}$ & $\mathrm{H}_{\mathrm{m}} \mathrm{h}_{\mathrm{m}}$ & $h_{m}$ & $\mathrm{H}_{\mathrm{m}} \mathrm{h}_{\mathrm{m}}$ & $h_{m}$ & $\mathrm{H}_{\mathrm{m}} \mathrm{h}_{\mathrm{m}}$ \\
\hline$x_{a t}=0,42$ & 21,18 & $\begin{array}{ll}8 & 8,9 \\
\end{array}$ & 17,35 & $\begin{array}{ll}5 & 7,3 \\
\end{array}$ & 13,90 & 5,8 & 10,42 & 4,4 & \begin{tabular}{|l|l}
6,84 \\
\end{tabular} & 2,9 & 3,36 & 1,4 & & & & \\
\hline $\mathrm{X}_{\mathrm{bt}}=11,9$ & 15,77 & 787,7 & 11,22 & $\begin{array}{ll}2 & 133,5 \\
\end{array}$ & 7,35 & $\begin{array}{ll}5 & 87,5 \\
\end{array}$ & \begin{tabular}{|l|l} 
& 3,67
\end{tabular} & 43,7 & & & & & & & & \\
\hline $\mathrm{X}_{\mathrm{et}}=-14,2$ & 0,560 & & 0,770 & $0-10,1$ & 0,818 & $8-11,6$ & 0,880 & $-12,5$ & 0,925 & $-13,1$ & 0,973 & $3-13,8$ & 0,997 & $\begin{array}{l}7-14,2 \\
\end{array}$ & & $0-14,2$ \\
\hline für $\mathrm{t}=+7,5^{\circ}$ & & $+188,6$ & & $+130,7$ & & $+81,7$ & & $+35,6$ & & $-10,2$ & & $-12,4$ & & $-14,2$ & & $-14,2$ \\
\hline für $t=-7,5^{\circ}$ & & $-188,6$ & & $-130,7$ & & $-81,7$ & & $-35,6$ & & $+10,2$ & & $+12,4$ & & $+14,2$ & & $+14,2$ \\
\hline - & & & & & & & Sum & & & & & & & & & \\
\hline mit $+\mathrm{t}$ & & -1940 & & -574 & & +152 & & +160 & & +49 & & +36 & & -59 & & -37 \\
\hline mit $-t$ & & -2317 & & -836 & & -12 & & +88 & & $+\quad 69$ & & +60 & & -31 & & -9 \\
\hline
\end{tabular}

$\mathrm{Zu}$ den vorstehend berechneten Momenten punkte von der Ausgleichebene und ist in folgentritt noch der Einule zelnen Querschnitte. Dieser Einfluß ist gleich Ausgleichebene entnommen sind, s. fig. AbStützlinienkraft mal Abstand der Flächenschwer- schnitt).

\begin{tabular}{|c|c|c|c|c|c|c|c|c|c|}
\hline & & 0 & 1 & 2 & 3 & 4 & 5 & 6 & 7 \\
\hline $\begin{array}{l}\text { Stützlini } \\
\text { Exzentrizität }\end{array}$ & $\begin{array}{l}\text { ienkraft } \mathrm{K}= \\
\text { (nach außen } \\
\text { positiv) } \mathrm{e}=\end{array}$ & $\begin{array}{r}1200 \\
+0,75\end{array}$ & $\begin{array}{r}1040 \\
+0,25\end{array}$ & $\begin{array}{r}980 \\
-0,02\end{array}$ & $\begin{array}{r}810 \\
-0,10\end{array}$ & $-0,06$ & $\begin{array}{r}700 \\
\pm 0\end{array}$ & $\begin{array}{r}690 \\
+0,03 \\
\end{array}$ & $+0,06 \mathrm{~m}$ \\
\hline & $\mathrm{M}=\mathrm{Ke}=$ & +900 & +260 & -20 & -81 & -47 & \pm 0 & +20 & $+41 \mathrm{r}$ \\
\hline
\end{tabular}

Diese Werte sind zu den vorstehend ermittel- senkrecht zu dieser - mit den zugehörigen Exzenten zu addieren $1 \mathrm{~m}$ Querschnite 0 ist jedoch noch - tizater $900-72+217=1045$ ponenten $K_{0}$ und $K_{k}$ - nach weiterer $Z$ eflegung Damit ergeben sich als ungunstigste Momente senkin Komponenten in der Querschnittsebene und recht zur Ausgleichebene

\begin{tabular}{|c|c|c|c|c|c|c|c|c|}
\hline & 0 & 1 & 2 & 3 & 4 & 5 & 6 & 7 \\
\hline $\begin{array}{l}\text { ohne Wärmewirkung } \\
\text { mit }+7,5^{\circ} . \\
\text { mit }-7,5^{\circ}\end{array}$ & $\begin{array}{l}-1084 \\
-\quad 895 \\
-1272\end{array}$ & $\begin{array}{l}-445 \\
-314 \\
-576\end{array}$ & $\begin{array}{l}+\quad 50 \\
+\quad 32 \\
-\quad 32\end{array}$ & $\begin{array}{l}+43 \\
+79 \\
+\quad 7\end{array}$ & $\begin{array}{l}+12 \\
+\quad 2 \\
+22\end{array}$ & $\begin{array}{l}+48 \\
+36 \\
+60\end{array}$ & $\begin{array}{l}-26 \\
-40 \\
-12\end{array}$ & $\begin{array}{l}+18 \\
+4 \\
+32\end{array}$ \\
\hline
\end{tabular}

d) Ermittlung der Torsionsmomente.

Die Momente sind in der Übersicht auf S. 20 Die Torsionsmomente sind aus den Momenten 作 Wirkungen der X-Kräfte zusammengesetzt worden. schnitten $0,1,2, \ldots$, in denen die gekrümmte in $\mathrm{X}_{\mathrm{e}}$ ist zu beachten, daß ein Moment $\mathrm{X}_{\mathrm{e}}=-1$ wirkhthe Stabachse zu dem gebrochenen

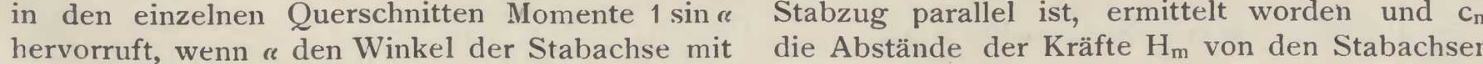
der Wagerechten bedeutet. 


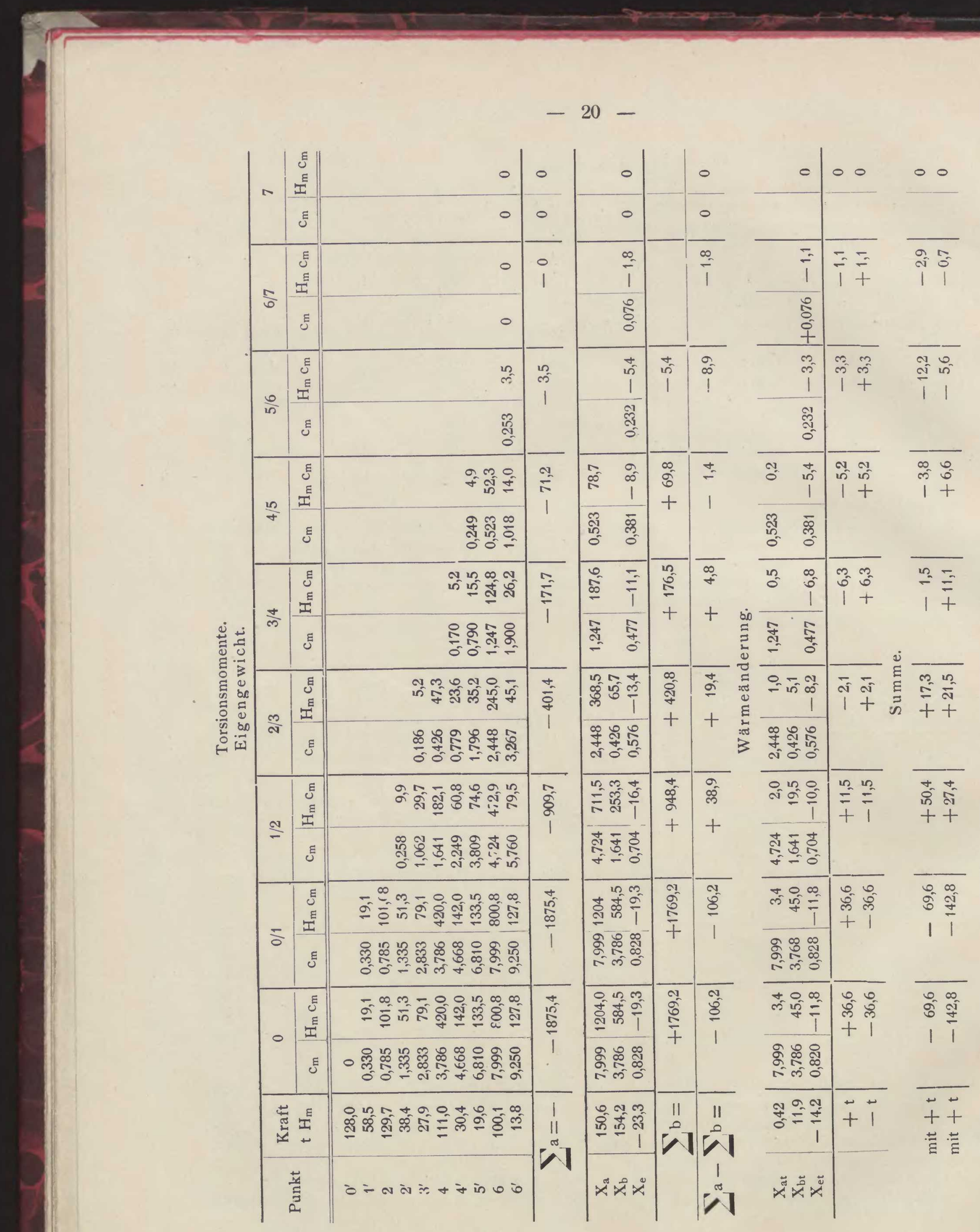




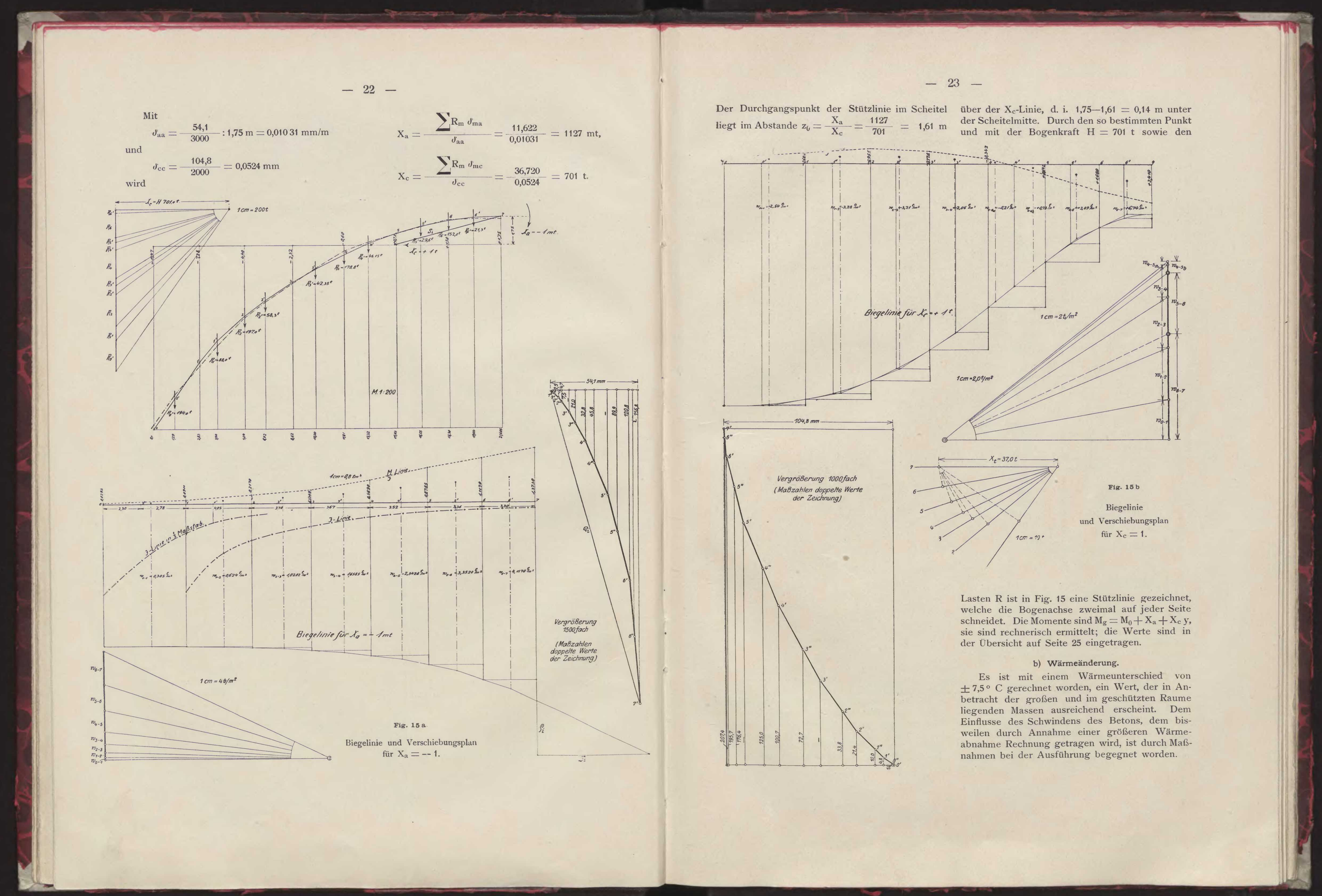




\section{$-24-$}

Die Wärmebogenkraft ergibt sich zu

dann eine gegenseitige senkrechte Verschiebun

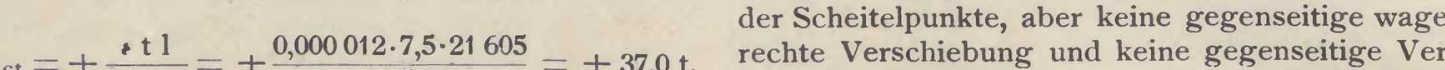

Durch Projektion auf die Stabachsen erhält man die Stabmit y die Momente Mutipie Werte sind in der Übersicht auf Seite 25 eingetragen

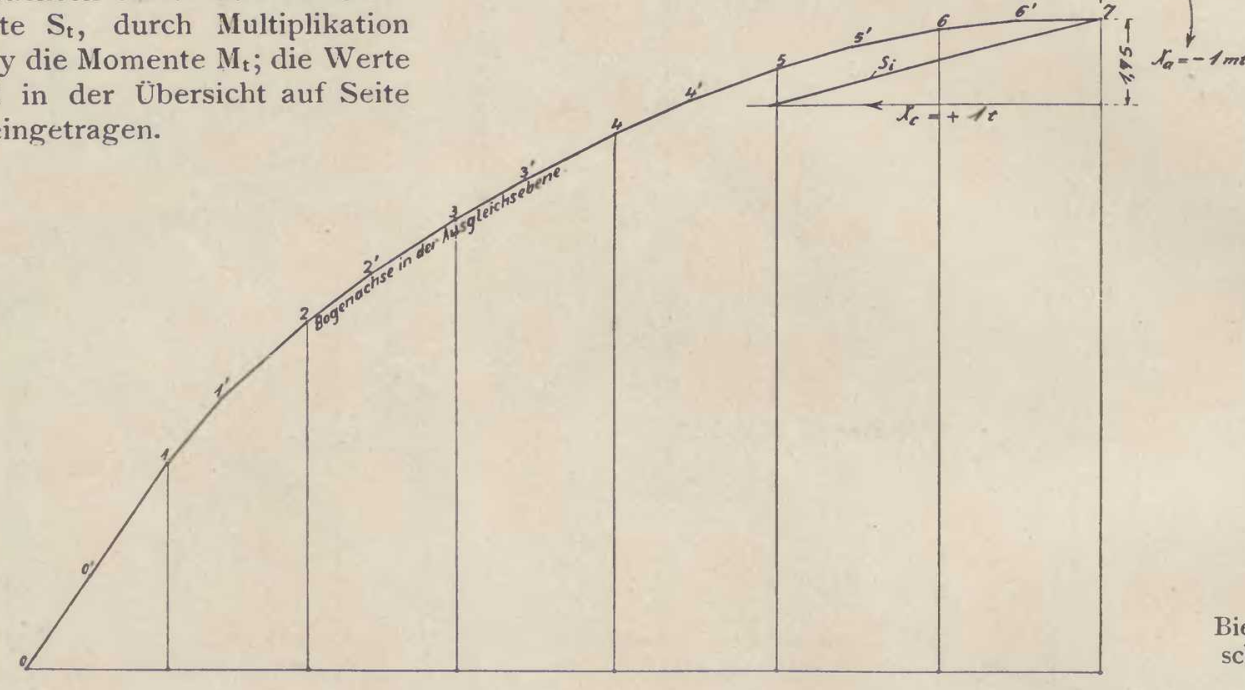
Biegslinie und Ver-
schiebungsplan fuir
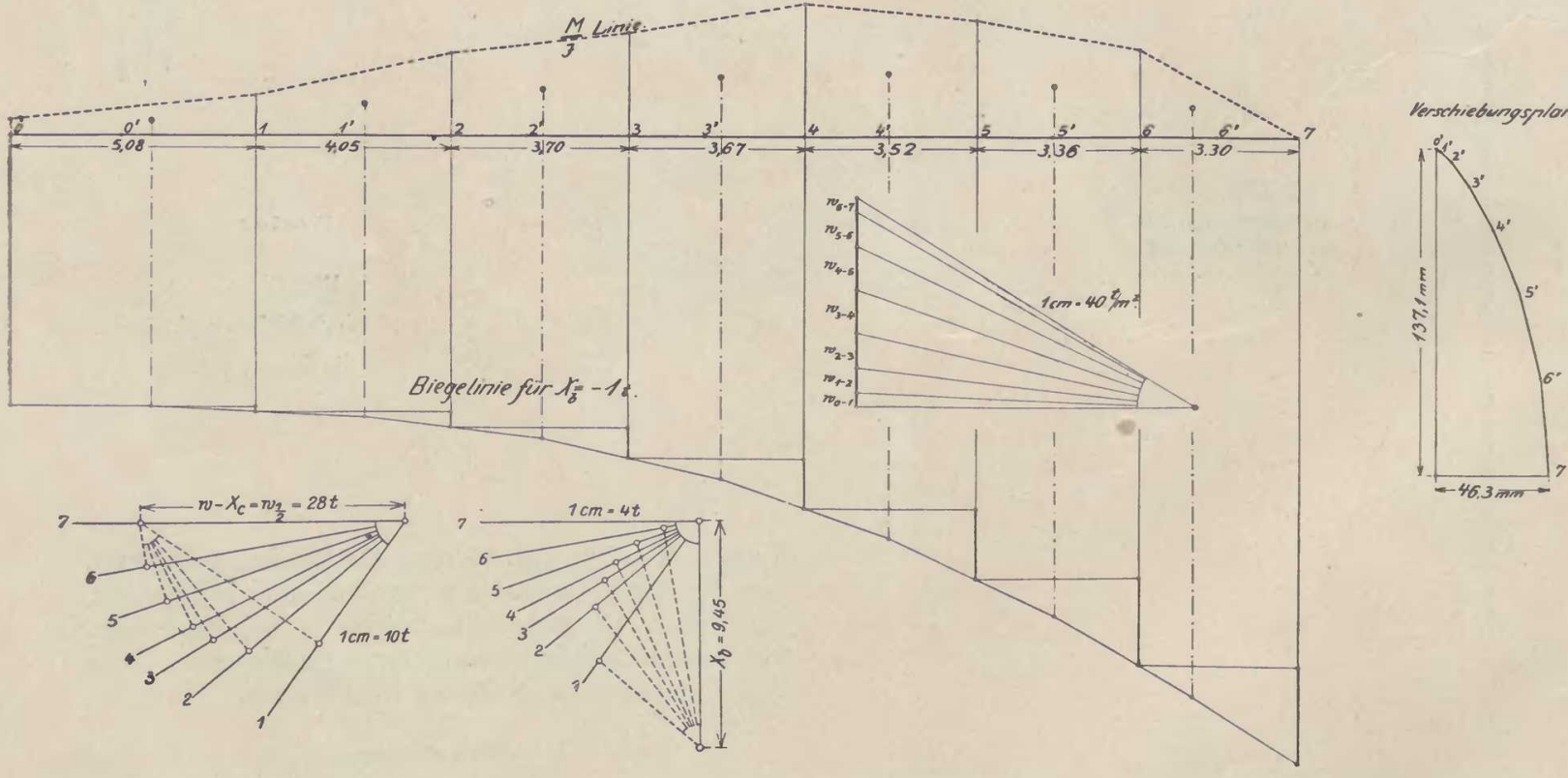

c) Windkräfte. bekanntę̄Größe $X_{b}$, die Querkraft, auf. Mit de Der Winddruck auf eine Kuppelhälfte beträgt in $\mathrm{W}=56 \mathrm{t}$. Es ist die ungünstige Annahme ge- $\mathrm{w}$ ist die Biegelinie und aus dieser der macht worden, dals diese Kraft in voller Hohe im versch de $\begin{array}{ll}\text { Bogenscheitel in den Hauptbogen geleitet wird. } & \delta_{\mathrm{wb}}=J_{\mathrm{bw}}=46,3: 200=0,2315 \mathrm{~mm}, \delta_{\mathrm{bb}}= \\ \text { Nan läßt nun je de Hälfte, also } 28 \mathrm{t} \text { am linken } & 2 \cdot 137,1: 200=1,371 \mathrm{~mm} \text { sich ergibt (s. Fig. 16). }\end{array}$ und am rechten Kragarme angreifen, sie ergeben Damit wird
$-25-$

$\mathrm{X}_{\mathrm{bw}}=\frac{\mathrm{W} \delta_{\mathrm{wb}}}{\delta_{\mathrm{bb}}}=\frac{56 \cdot 0,2315}{1,371}=9,45 \mathrm{t}$.

Das Windmoment für einen Bogenpunkt $m$ ist

$$
\mathrm{M}_{\mathrm{w}}=-\frac{\mathrm{W}}{2} \mathrm{y}_{0}+\mathrm{X}_{\mathrm{bw}} \mathrm{x},
$$

die Stabkräfte sind

$S_{w}=+\frac{W}{2} \cos \kappa+X_{b} \sin \kappa$,

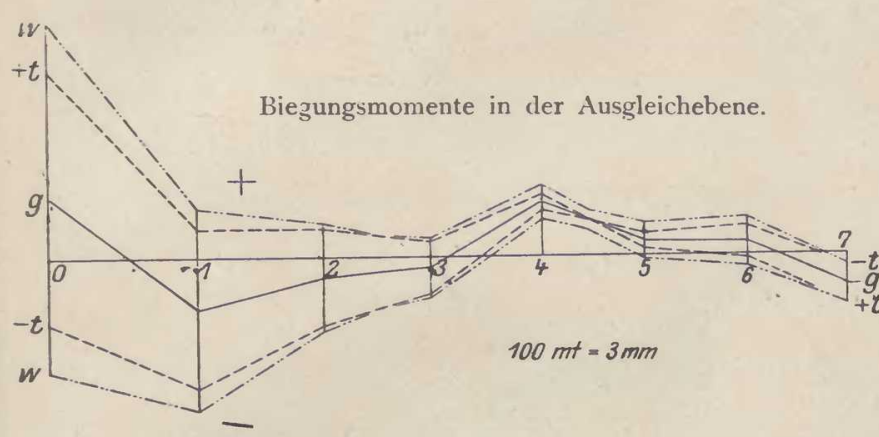

\begin{tabular}{|c|c|c|c|c|c|c|}
\hline & \multicolumn{2}{|c|}{ Eigengewicht } & \multicolumn{2}{|c|}{ Wind } & \multicolumn{2}{|c|}{$\begin{array}{l}\text { Wärme- } \\
\text { zunahme }\end{array}$} \\
\hline & $\begin{array}{c}\mathrm{M} \\
\mathrm{mt}\end{array}$ & $\begin{array}{l}S \\
t\end{array}$ & $\begin{array}{c}\mathrm{M} \\
\mathrm{mt}\end{array}$ & $\begin{array}{l}S \\
t\end{array}$ & $\begin{array}{l}\mathrm{M} \\
\mathrm{mt}\end{array}$ & $\begin{array}{l}S \\
t\end{array}$ \\
\hline & & & & & & \\
\hline & $-172,5$ & 1075 & $\mp 75,5$ & $干_{23}$ & $\begin{array}{r}+269,0 \\
+\end{array}$ & \\
\hline & $-62,5$ & 880 & $\mp 21,0$ & $\mp 27$ & $+163,0$ & +3 \\
\hline & $-39,0$ & 845 & $\pm 9,0$ & $\mp 29$ & $+86,0$ & $+i$ \\
\hline & $+180,0$ & 746 & $\pm 26,5$ & 干 29 & $+22,0$ & + \\
\hline & $+47,5$ & 732 & $\pm 33,0$ & \pm 29 & $-2.2,5$ & \\
\hline & $\begin{array}{l}+\quad 41,0 \\
-101,0\end{array}$ & 701 & & $(\mp 28)$ & $\begin{array}{l}- \\
-65,0 \\
\end{array}$ & $\begin{array}{l}+37 \\
+37\end{array}$ \\
\hline
\end{tabular}
erơeben sich folgende Grenzwerte, Kräften $\mathrm{S}$ Wärmezunahme, $\mathrm{A}$ Wärmeabnahme bedeuten. Grenzwerte der Momente und Stabkräfte.

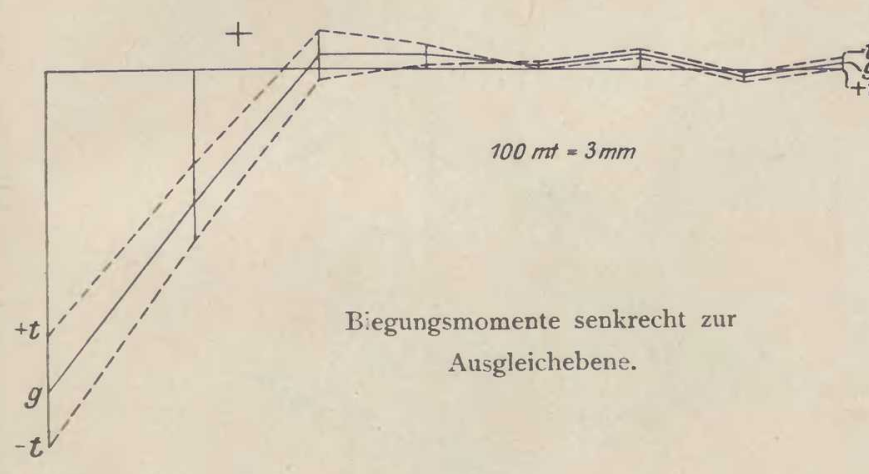

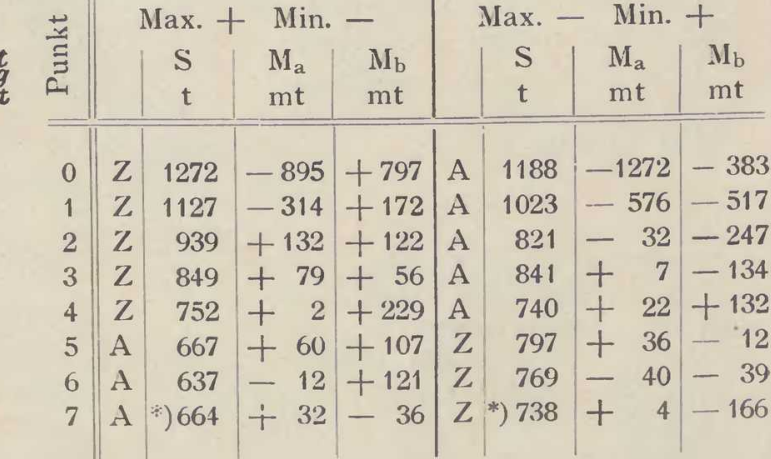

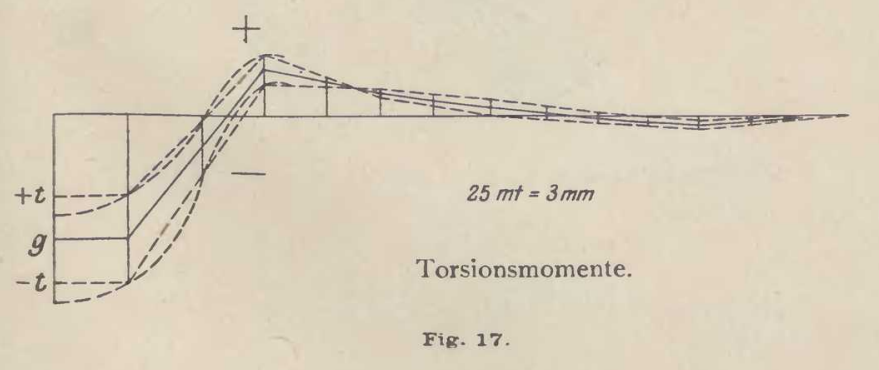

ür Wind von links nach rechts verringern sie auf kräfte und Momente sind in folgender Ubersicht eingetragen.

$$
\text { d) Zusammenfassung. }
$$

3. Spannungsermittlung a) Hauptbogen

c) Biegungsspannungen

Sowohl die Momente $\mathrm{M}_{\mathrm{b}}$ wie $\mathrm{M}_{\mathrm{a}}$ waren be zogen auf die Schwerpunktsachsen, und zwar Achse a parallel zur Ausgleichebene a a. Die Abande des Angriftspunktes der Normalkraft $\mathbf{S}$ vo

$$
\mathrm{a}=\frac{\mathrm{M}_{\mathrm{b}}}{\mathrm{S}}, \quad \mathrm{b}=\frac{\mathrm{M}_{\mathrm{a}}}{\mathrm{S}} .
$$

Sind nun $x_{a}$ und $y_{a}$ die Koordinaten dieses Angriffspunktes bezogen auf die Hauptachsen des

Die Einzelmomente sind in folgende Cbersicht eingetragen und in Fig. 17 dargestellt. druck fort. 
26

Querschnittes, so sind die Achsabschnitte der

$x_{n}=-\frac{J_{y}}{F_{x_{a}}}, \quad y_{n}=-\frac{J_{x}}{F_{y_{a}}}$

bezeichnen endlich $\mathrm{x}$ und $\mathrm{y}$ die Koordinaten eines beliebigen Punktes in bezug auf die Hauptachsen,

$$
\sigma=\frac{\mathrm{S}}{\mathrm{F}}+\frac{\mathrm{S} \mathrm{x}_{\mathrm{a}} \mathrm{x}}{\mathrm{J}_{\mathrm{y}}}+\frac{\mathrm{S} \mathrm{y}_{\mathrm{a}} \mathrm{y}}{\mathrm{J}_{\mathrm{x}}}
$$
Spannungen für die 3 Zustände

a) Eigengewicht, Warmezunahme, Wind,

b) " Wärmeabnahme,

c) allein
ermittelt worden. Im folgenden sind die Berechnungen für den
Kämpfer- und den Scheitelquerschnitt wiedergegebe Zu den Werten in der letzwirkung der Pfostenkraft $K_{0}$ (s. S. 19), welche eine Stabkraft $\mathrm{K}_{0}=183 \mathrm{t}$ und ein Moment
$\mathrm{M}_{\mathrm{b}^{\prime}}=-183 \cdot 0,41=-75 \mathrm{mt}$ gibt, so daß man folgende
Werte erhält: Fall a).
$\mathrm{M}_{\mathrm{a}}=-895 \mathrm{mt}$, $\mathrm{M}_{\mathrm{a}}=-895 \mathrm{mt}$,
$\mathrm{M}_{\mathrm{b}}=+772 \mathrm{mt}$, $\mathrm{S}=1455 \mathrm{t}$, $\mathrm{a}=\frac{\mathrm{M}_{\mathrm{b}}}{\mathrm{S}}=\frac{722}{1455}=0,496$, $\mathrm{b}=\frac{\mathrm{M}_{\mathrm{a}}}{\mathrm{S}}=-\frac{895}{1455}=-0,615 ;$ aus der Fig. 18 ergibt sich $\mathrm{x}_{\mathrm{a}}=-0,74, \quad \mathrm{y}_{\mathrm{a}}=-0,31$ Für die Nullinie ergibt sich $\mathrm{x}_{\mathrm{n}}=-\frac{\mathrm{J}_{\mathrm{y}_{\mathrm{y}}}}{\mathrm{F}_{\mathrm{x}}}=-\frac{27,20}{22,05(-0,74)}=+1,67$, $y_{n}=-\frac{J_{x}}{F_{y_{a}}}=-\frac{59,20}{22,05(-0,31)}=+8,65$. Die Maximaldruckspannung tritt im Eckpunkte
$C_{0}(x=-2,98, y=+1,04)$ auf mit $\sigma={ }_{22,05}^{1455}+\frac{1455(-0,74)(-2,98)}{27,20}+\frac{1455(-0,31) 1,04}{59,20}$

$=+65,9+118,0-7,9$

$=-176,0 \mathrm{t} / \mathrm{qm}$

\section{(1)}

Die Maximalzugspannung tritt in $\mathrm{B}_{0}(\mathrm{x}=+1,41$ $y=+3,68)$ auf $\mathrm{mit}$

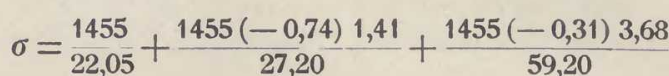

$=+65,9-55,7-28,1$

$=-17,9 \mathrm{t} / \mathrm{mm}$

\section{Fall b).}

$\mathrm{M}_{\mathrm{a}}=-1272 \mathrm{mt}, \quad \mathrm{M}_{\mathrm{b}}=-458 \mathrm{mt}, \quad \mathrm{S}=1371$ $\mathrm{a}=-0,334, \quad \mathrm{~b}=-0,927$, $\mathrm{x}_{\mathrm{b}}=-0,16, \quad \mathrm{y}_{\mathrm{b}}=-0,97$ $\mathrm{x}_{\mathrm{n}}=+7,71, \quad \mathrm{y}_{\mathrm{n}}=+2,7$

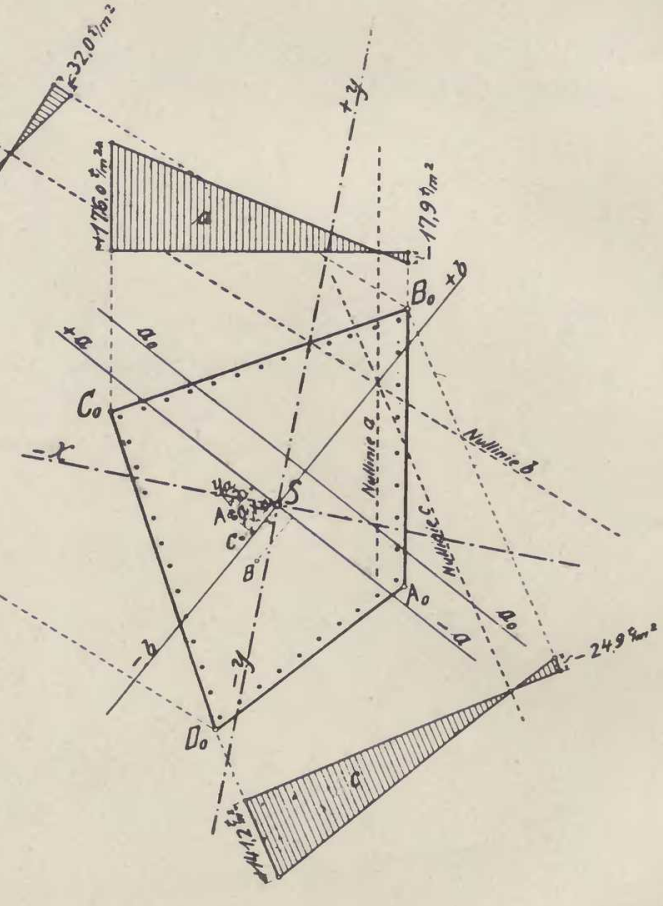

Maximaldruckspannung in $D_{0} \quad(x=-0,25$ $=-3,8 \%) \quad \sigma=+151,7 \mathrm{t}^{\prime} \mathrm{qm}$

Maximalzugspannung in $\quad \mathrm{B}_{0} \quad(\mathrm{x}=+1,41$, $\mathrm{y}=+3,68) \quad \sigma=-9,1 \mathrm{t} / \mathrm{qm}$. Fall c).

$\mathrm{M}_{2}=-1084 \mathrm{mt}, \quad \mathrm{M}_{\mathrm{b}}=+132 \mathrm{mt}, \quad \mathrm{S}=1413 \mathrm{t}$ $\mathrm{a}=+0,093, \quad \mathrm{~b}=-0,767$, $\mathrm{x}_{\mathrm{c}}=-0,44, \quad \mathrm{y}_{\mathrm{c}}=-0,64$ $x_{\mathrm{n}}=+2,81, \quad \mathrm{y}_{\mathrm{n}}=+4,19$

$-27-$

Der Querschnitt ist bewehrt mit Rundeisen von $30 \mathrm{~mm} \Phi$ in rd. $0,55 \mathrm{~m}$ Abstand, der erfordetliche und der vorhandene Eisenquerschnitt betraven für die drei

a) Zugfläche: Trapez von i. M. 4,70 m Länge und $0,47 \mathrm{~m}$ Höhe,

Zugkraft: $4,70 \cdot 0,47 \frac{17,9}{2}=19,8 \mathrm{t}$,

vorhanden 9 Rundeisen mit 63,6qcm Querschnitt,

b) Zugfläche: Dreieck von 1,90 m Länge und $1,35 \mathrm{~m}$ Höhe,

Zugkraft: $\frac{1,90 \cdot 1,35}{2} \cdot \frac{32,0}{3}=13,6 \mathrm{t}$,

vorhanden 6 Rundeisen mit $42,4 \mathrm{qcm}$ Querschnits,

c) Zugfläche: Dreieck von 2,20 m Länge und $0,95 \mathrm{~m}$ Höhe,

Zugkraft: $\frac{2.20 \cdot 0,95}{2} \cdot \frac{24,9}{3}=8,7 \mathrm{t}$,

vorhanden 6 Rundeisen mit 42,4 qcm Querschnitt.

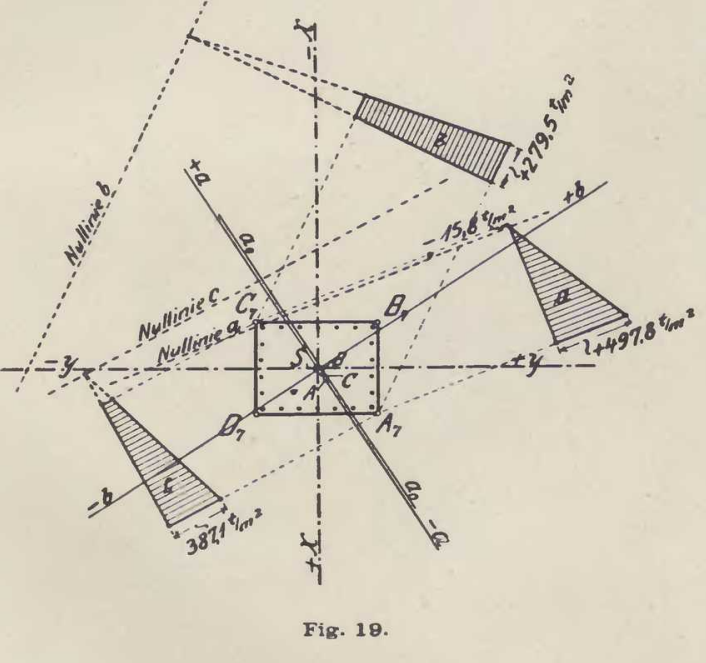

Querschnitt 7

Fall a).

$\mathrm{M}_{\mathrm{a}}=+4 \mathrm{mt}, \quad \mathrm{M}_{\mathrm{b}}=-166 \mathrm{mt}, \quad \mathrm{S}=738 \mathrm{t}$ $\mathrm{a}=-0,225, \quad \mathrm{~b}=+0,005$, $\mathrm{x}_{\mathrm{a}}=+0,18, \quad \mathrm{y}_{\mathrm{a}}=+0,12$, $\sigma_{\mathrm{A}}=+497,8 \mathrm{t} / \mathrm{qm}, \quad \sigma_{\mathrm{C}}=-15,8 \mathrm{t} / \mathrm{qm}$ Fall b). $\mathrm{M}_{\mathrm{a}}=+32 \mathrm{mt}, \quad \mathrm{M}_{\mathrm{b}}=-36,0 \mathrm{mt}, \quad \mathrm{S}=664 \mathrm{t}$,
$\sigma_{\mathrm{A}}=+279,5 \mathrm{t} / \mathrm{qm}, \quad \sigma_{\mathrm{C}}=+154,5 \mathrm{t} / \mathrm{qm}$.
Fall c).

$\mathrm{M}_{\mathrm{a}}=+18 \mathrm{mt}, \quad \mathrm{M}_{\mathrm{b}}=-101 \mathrm{mt}, \quad \mathrm{S}=701 \mathrm{t}$

Der Querschnitt ist bewelrt mit 22 Rundeise von $20 \mathrm{~mm} \phi$, in den Ecken $30 \mathrm{~mm} \Phi$, so daß für fung vorhanden ist (s. Fig. 19)

$$
\text { B) Torsionsspannungen. }
$$

Die auf S. 20 ermittelten Torsionsmomente sind in Fig. 17 aufgetragen worden, wobei das Vieleck durch eine durch dessen Ecken gehende ind wie bei den Biegunosmomenten (s. S. 19) auch hier die Wirkungen der Lasten $\mathrm{P}_{\mathrm{o}^{\prime}}$ und $\mathrm{K}_{\mathrm{o}}$ zu berücksichtigen, welche nach ihrer Zerlegung in Komponenten in der Querschnittsebene und höhen auf

$-160+49-51-146=-308 \mathrm{tm}$

Die größte Schubspannung tritt bei einem
chtecke in der Mitte der laingeren Seite auf $t=\frac{9 \mathrm{M} 9}{2 \mathrm{~b}^{2} \mathrm{~h}}$.

an erhält folgende Werte für die am stärksten beanspruchten Querschnitte

$$
\begin{array}{ccccc}
\text { Querschnitt } & 0 & 1 & 2 & 6 \\
\hline M_{9} & -308 & -65 & +50 & -15 \mathrm{mt}, \\
I_{\max } & 17,3 & 8,8 & 11,1 & 12,4 \mathrm{t} / \mathrm{qm} .
\end{array}
$$

Obgleich diese Schubspannungen weit unter dem zulässigen Werte von $4,5 \mathrm{~kg} / \mathrm{qcm}$ liegen, so sind doch in den Bogentach ist in der Ansichtsflezch fallend ron links oben nach rechts unten für negative Momente (s. Fig. 20). Die Bewehrung des Hauptbogens ist in Fig. 21 dargestellt, die Eiseneinlagen sind ist in Gre 21 dare schiedener Mischung von 1:6:8 bis 1:3:3 je nach den auftretenden Beanspruchungen hergestellt.

b) Strebenbinder.

Auf S. 17 sind die Kräfte $X_{a}$ und $X_{b}$ zu 150,6 and $154,2 \mathrm{t}$ aus Eigengewicht, $\mathrm{zu} 0,4 \mathrm{t}$ und $11,9 \mathrm{t}$ $151,0 \mathrm{t}$ und $166,1 \mathrm{t}$ ermittelt worden. Diesen in den Normalen zur Ausgleichebene wirkenden Kräften entsprechen obere Kämpferkräfte von $\mathrm{K}_{\mathrm{a}}{ }^{\circ}$ $=156 \mathrm{t}$ und $\mathrm{K}_{\mathrm{b}}{ }^{\circ}=172 \mathrm{t}$, dir has der teten der bauten erreben sich untere Kämpferkräfte $\mathrm{K}_{\mathrm{u}} \mathrm{u}-$ $220 \mathrm{t}$ und $\mathrm{K}_{\mathrm{b}}{ }^{\mathrm{u}}=225 \mathrm{t}$ (s. Fig. 13 fur Binder II). Es war jedoch verlangt, daß die Strebenbinder aucl 


$$
-28-
$$

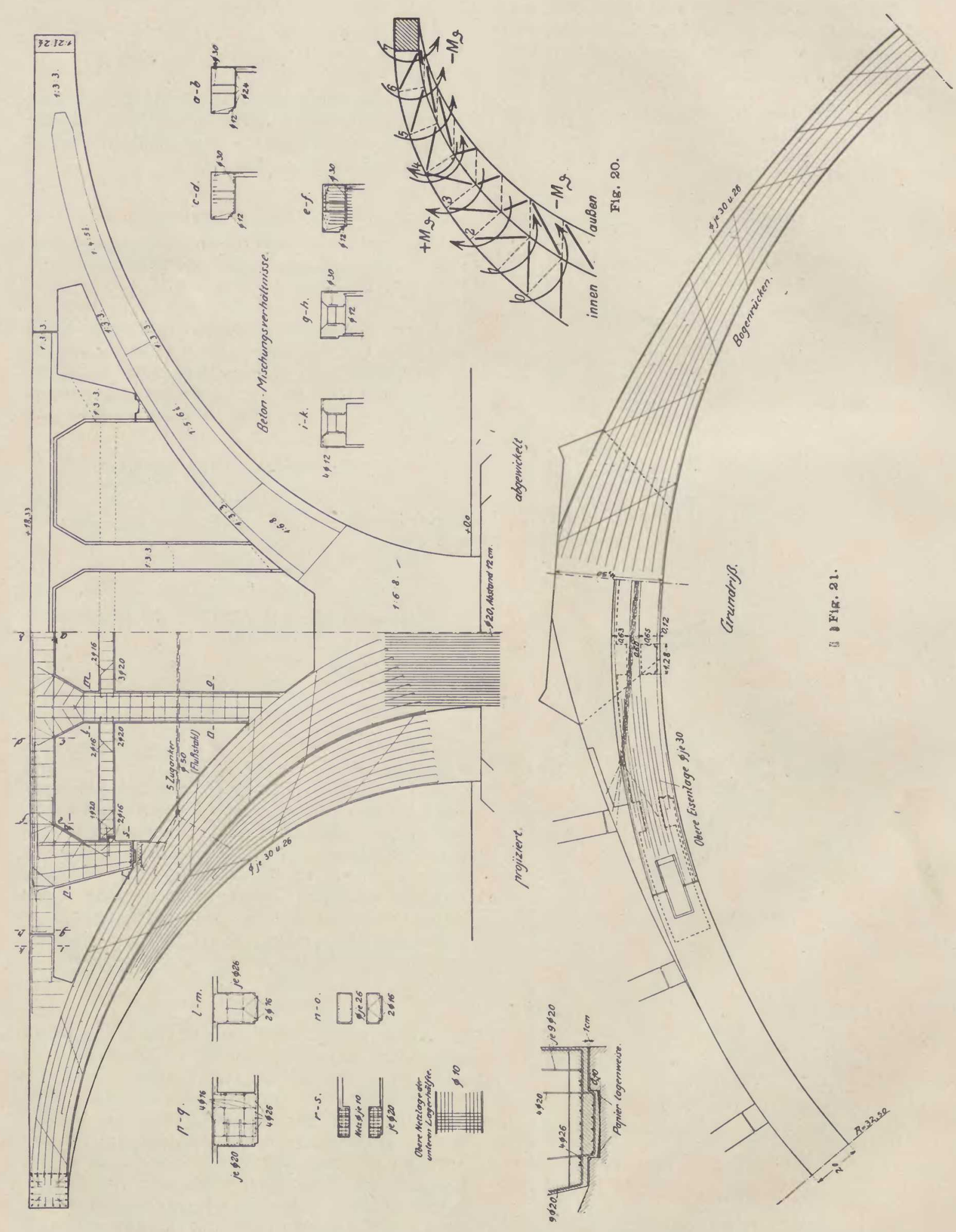

$-29-$ dann noch nicht überbeansprucht werden sollten,
wenn durch unvorhen die größßten Kräfte und Momente in den
wene Ereignisse die voll- Strebenbindern. kommene Einspannung der Hauptbogen in den kräf- Im Strebenbinder I wird der ungünstigste $\begin{array}{ll}\text { tigen Eckpfeilern vermindert oder durch stärkere } & \text { Querschnitt II-II (s. Fig. 22) durch eine Kraft P } \\ \text { Rissebildung sogar vollkommen aufgehoben sein } & =234 \mathrm{t} \text { in } 0,17 \mathrm{~m} \text { Abstand von der inneren Laibung }\end{array}$

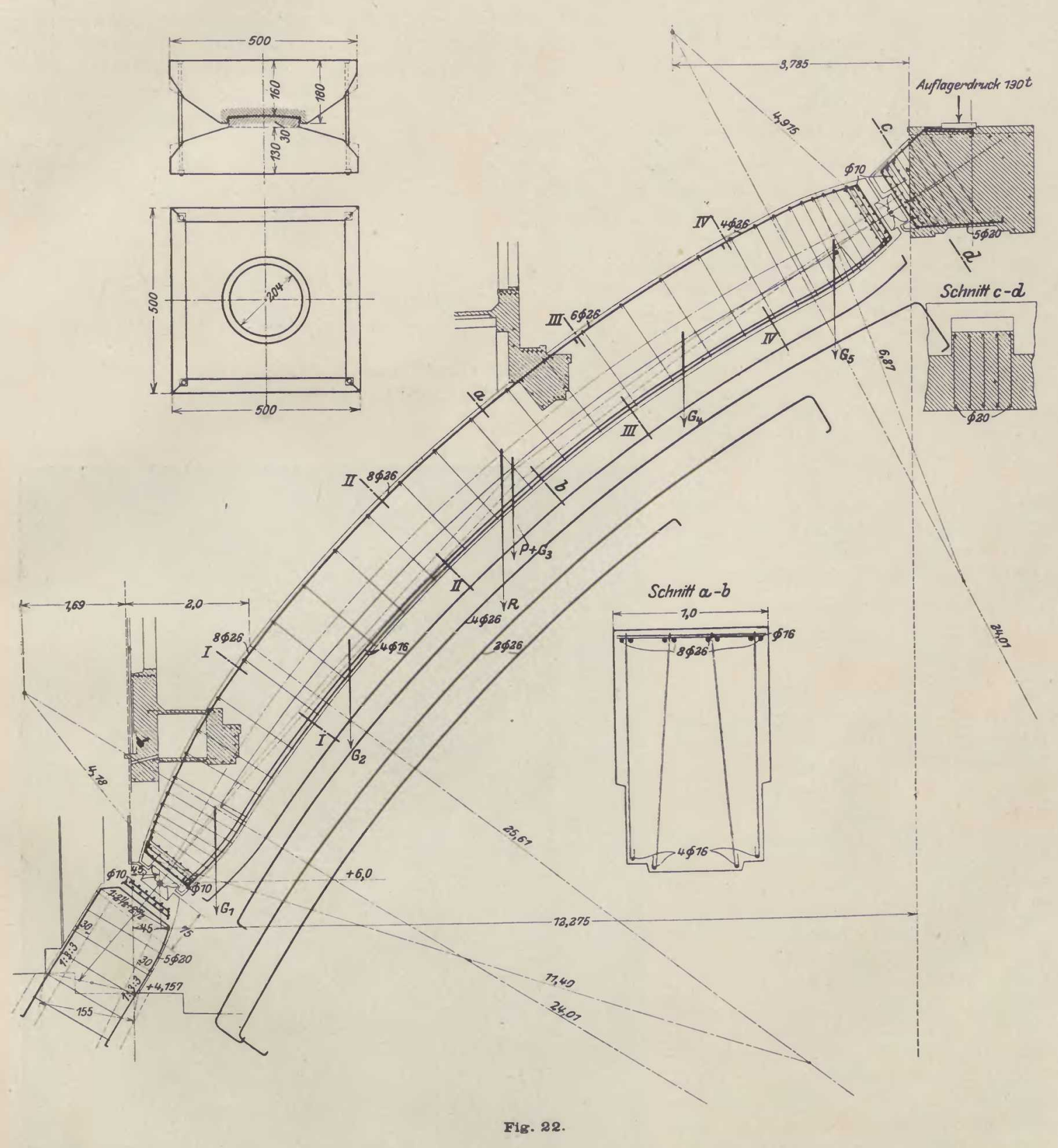

sollte. Die Strebenbinderkräfte ergeben sich dann beansprucht, der Querschnitt besitzt eine Druckangenahert durch Bildung der statischen Momente bewehrung dieser sowie der Has neu gebildete Kämpfergelenk des Haupt- $26 \mathrm{~mm} \phi=42,5 \mathrm{qcm}$. Nach der genauen Bebogens, sie betragen $\mathrm{K}_{\mathrm{a}} \mathrm{a}^{\prime}=190 \mathrm{t}, \mathrm{K}_{\mathrm{b}^{\circ}}=206 \mathrm{t}$ und rechnung unter Beriucksichtigung der Doppel- 


\section{$-30-$} bewehrung ergeben sich die Beanspru,
$\sigma_{\mathrm{b}}=60,5 \mathrm{~kg} / \mathrm{qcm}$ Druck,
$\sigma_{\mathrm{e}^{\prime}}=880$
$\sigma=605$

$\sigma_{\mathrm{e}}=605 \stackrel{n}{n}$ Zug.
Im Strebenbinder II beträgt die größte Kraft

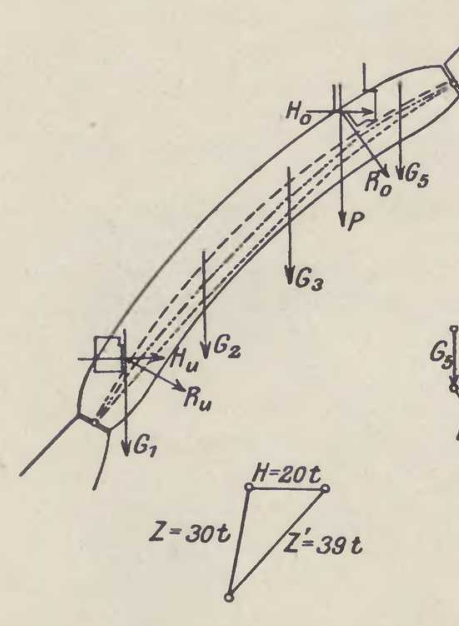

Fig. 23.
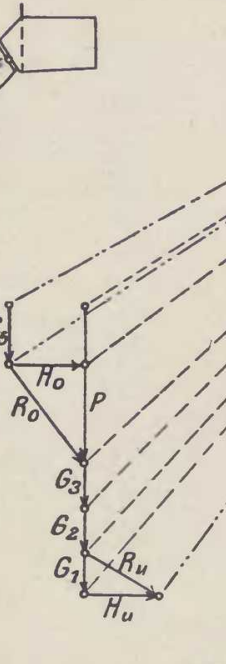

$240 \mathrm{t}$ in $0,18 \mathrm{~m}$ Abstand, der Querschnitt ist bewehrt mit 4 Druckeisen
von $16 \mathrm{~mm} \Phi=8,04 \mathrm{qcm}$ und $9 \mathrm{Z}$

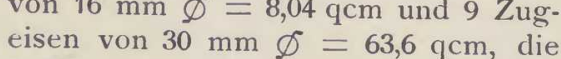
eisen von $30 \mathrm{~mm}, \Phi=63,6$
Beanspruchungen betragen

$\sigma_{\mathrm{b}}=54,8 \mathrm{~kg} / \mathrm{qcm}$ Druck,

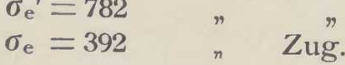

Es ist somit eine Zug. Zugbewehrung in diesen besonders wichtigen Gliedern vorgesehen, der
Beton ist ein Granitfeinschotterbeton Beton ist ein Granitfeinschotterbeto
1:3:3 von sehr großer Festigkeit. Die Versteifungsbänder der Stre-
Diestign benbinder haben Eiseneinlagen vo
solcher Größe erhalten, daßß sie den elastischen Verbiegungen der Exzentrizität der Stützlinie infolge der Bändern auftretenden Zugkräfte kônnen nun hochstens so groß̧ werpunkte in linie bis etwa in die drückt (s. Fig. 23). Hierzu sind wagerechte Kräfte von etwa $20 \mathrm{t}$ nötig funcsbündorn Zuglentte $40 \mathrm{t}$ auftreten. Außer der für Figengewicht erforderlichen Bewehrung
sind zur Aufnahme dieser Zugkräfte

im unteren Bande 5 Eisen von $28 \mathrm{~mm} \quad \varnothing=30,8$ cm, im oberen 7 Stück von $28 \mathrm{~mm} \not \varnothing=43,1 \mathrm{qcm}$ Die Strebenbinder sind an Apsidenpfeilern mit Stablgußkugellagern angeschlossen, deren obere für $200 \mathrm{t}$, deren untere für $250 \mathrm{t}$ Druck bei $100 \mathrm{~kg} / \mathrm{qcm}$ sind und Betonbeanspruchung Abmessungen von $45 \cdot 45 \mathrm{~cm}$ und $50.50 \mathrm{~cm}$ besitzen. Die Beanspruchung in den Kugel-
flachen beträgt nach Hertz

$\sigma=-\frac{3}{2 \pi} \sqrt[3]{\mathrm{A}\left(\frac{3}{2} \cdot \frac{\frac{1}{\mathrm{r}_{1}}-\frac{1}{\mathrm{r}_{2}}}{\frac{1}{\mathrm{E}_{1}}+\frac{1}{\mathrm{E}_{2}}}\right)^{2}}$

Mit $r_{1}=150 \mathrm{~cm}, r_{2}=190 \mathrm{~cm}, E_{1}=E_{2}=2200 \mathrm{t} / \mathrm{qcm}$ $\sigma=5,28 \mathrm{t} / \mathrm{qcm}$,

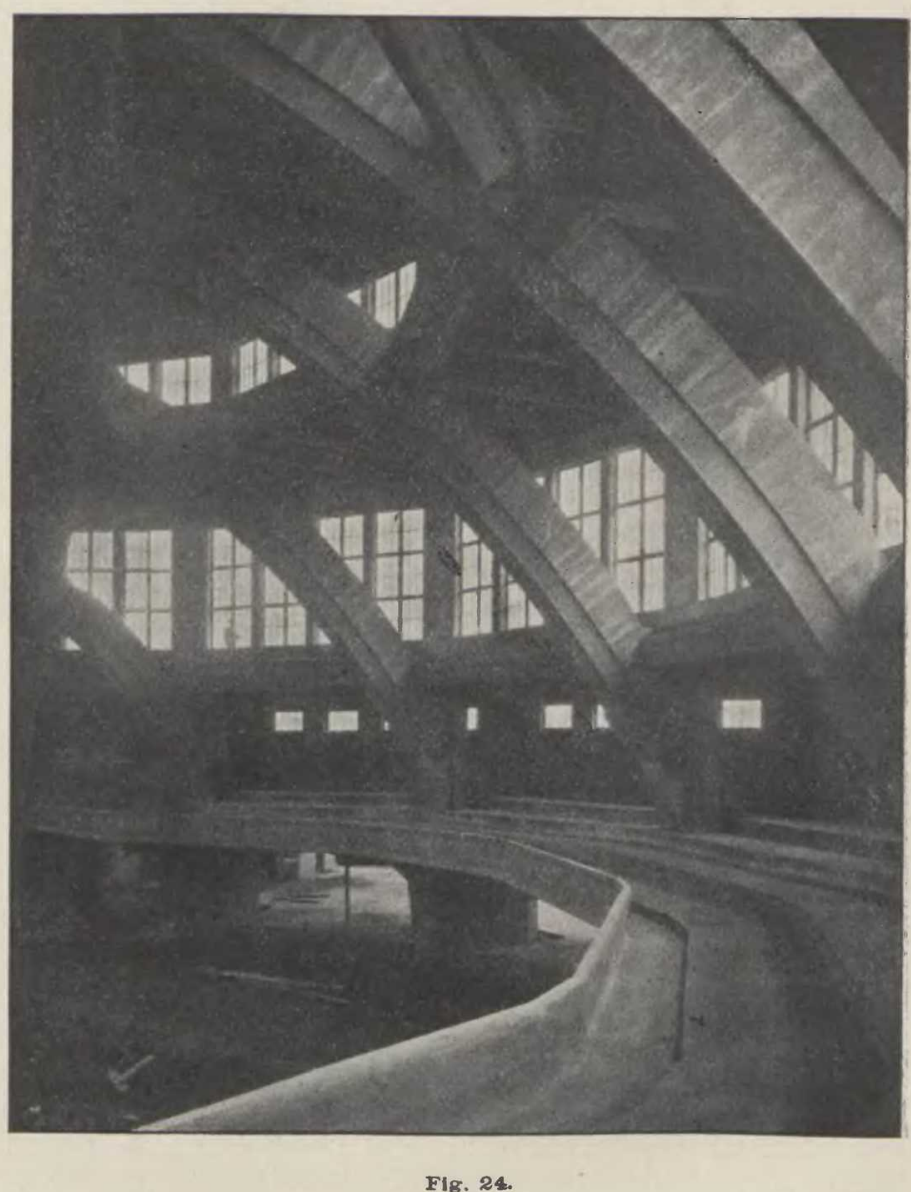

$-31-$

während die zulässige Grenze etwa 6 beträgt; die das Rahmeneigengewicht. Die geringe Krümmung im Grundrisse ist nicht in Rechnung gesetzt worden, der Rahmen vielmehr als ebenes Gebilde berechnet worden. Das im allgemeinen 7 fach

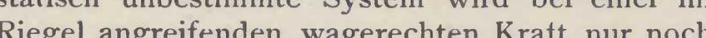
3 fach unbestimmt, für die Einwirkung des Eigen$\mathrm{d}=\sqrt{\frac{\mathrm{A} \cdot 4}{\sigma \cdot \pi}}=7,8 \mathrm{~cm}$

Die Höhe der Lagerteile in der Mitte ist nac der Formel für Platten mit Einzellast in der Mitte
und gleichmäßjig verteiltem Gegendruck ermittelt. gewichtes sind gewisse vereinfachende Annahmen $\sigma_{\max }=1,5, \frac{\mathrm{ab}}{\mathrm{a}^{2}+\mathrm{b}^{2}} \cdot \frac{\mathrm{A}}{\mathrm{s}^{2}}$

für $\mathrm{a}=\mathrm{b}$ wird

$$
\sigma_{\max }=0,75 \% \frac{\mathrm{A}}{\mathrm{s}^{2}}
$$

setzt man als zulässige Biegungsbeanspruchung $=1,500 \mathrm{t} / \mathrm{qcm}$, so wird die Stärke mit $\boldsymbol{\eta}=\mathbf{2 , 0 0}$

$$
\mathrm{s}=\sqrt{\frac{1,50 \cdot 250}{1,500}}=15,8 \mathrm{~cm},
$$

1. Einwirkung des Winddruckes. Als Hauptsystem wurde nicht ein statisch
bestimmtes System gewählt, sondern der mittlere Rahmen unter Annahme gelenkiger Lagerung, also en $Z$ weigelenkbogen. Dessen Bogenkraft X W und der 3 statisch unbestimmten Größen $Z$ $Z_{3}, Z_{3}$. Zur Ermittelung der Stützenwiderstände werden die Einwirkungen der Kräfte auf das Grundsystem getrennt untersucht, und zwar rechts, 3 das Kräftepaar ans den beiden 7 4. desgl. die beiden Momente $Z_{3}$. Die Stuitzengewählt wurden $16 \mathrm{~cm}$ in dem mittleren 28,4 * widerstände sind folgende (s. Fig. 25)

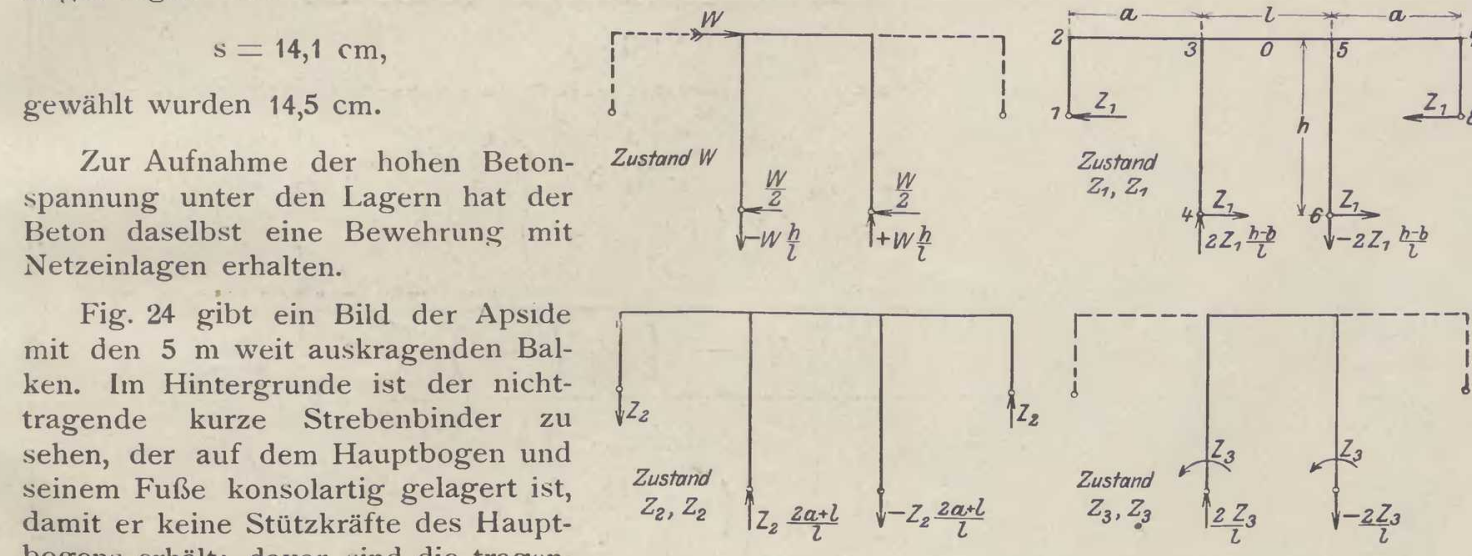

bogens erhält; davor sind die trage-

verkleidet sind, mit den krättigen Versteifung bändern.

\section{Windrahmen.}

(Nach der Berechnung von Dyckerhoff u. Widmann).

Bie vier Pfosten über den Eckpfeilern sind, wie bereits eingangs erwähnt, zu einem steifen
Rahmen, dem sogen. Windrahmen, zusammenRahmen, dem sogen. Whindahmen, zusammenAußennfosten celenkig auf den Haupthögen lagert sind. Dieser Rahmen wird beanspruch
durch die Kuppellasten, den Winddruck und

\begin{tabular}{c|c|c|c|c}
\hline & $\mathrm{H}_{\mathrm{a}}$ & $\mathrm{H}_{\mathrm{b}}$ & $\mathrm{A}$ & $\mathrm{B}$ \\
\hline $\mathrm{W}$ & $\frac{\mathrm{W}}{2}$ & $\frac{\mathrm{W}}{2}$ & $-\mathrm{W} \frac{\mathrm{h}}{\mathrm{l}}$ & $+\mathrm{W} \frac{\mathrm{h} b}{1}$ \\
$Z_{1}, Z_{1}$ & $\mathrm{Z}_{1}$ & $Z_{1}$ & $2 Z_{1} \frac{\mathrm{h}-\mathrm{b}}{\mathrm{l}}$ & $-2 Z_{1} \frac{\mathrm{h}-}{\mathrm{l}}$ \\
$Z_{2}, Z_{2}$ & - & - & $Z_{2} \frac{2 \mathrm{a}+1}{\mathrm{l}}$ & $-Z_{2} \frac{2 \mathrm{a}+1}{\mathrm{l}}$ \\
$Z_{3}, Z_{3}$ & - & - & $\frac{2 Z_{3}}{\mathrm{l}}$ & $-\frac{2 Z_{3}}{\mathrm{l}}$
\end{tabular}


$-32$

Durch Übereinanderlegen der 4 Belastungsden Kräfte (Fig. 26). Die 3 statisch unbestimmten Größsen $Z$ werden mittels der Arbeitsgleichung
unter Voraussetzung starrer Stützpunkte berechnet. Hierzu dienen unter Einführung gleichen Trägheitsmomentes und $g$
maß̧es die drei Gleichungen

$z_{2}$

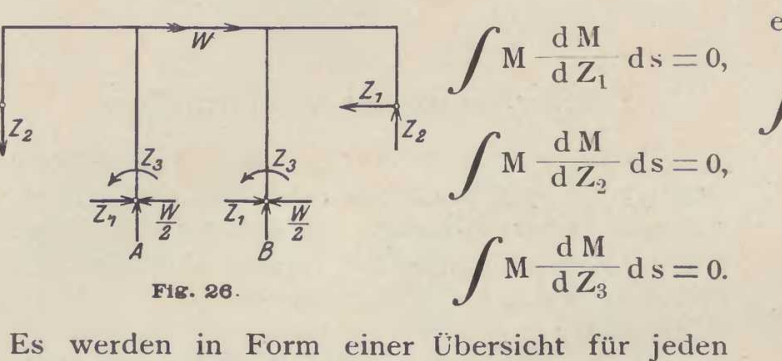
Es werden in Form einer Übersicht für jeden $w, M_{1}$ infolge $Z_{1}, M_{2}$ infolge $Z_{3}, M_{3}$ infolge $Z_{3}$, ferner $\frac{d M}{d Z}$ und $M \frac{d M}{d Z}$ je für $Z_{1}, Z_{2}$ und $Z_{3}$. In folgender Ubersicht sind als Beispiel nur die Whe $1 / 2$ r Werte für $3 / 4$ und vor allem $3 / 0$ sind fortgelassen.

$$
\int M \frac{d M}{d Z_{1}} \cdot d s
$$

für alle Stäbe zusammengeschrieben und Null (resetzt werden, desgleichen die Werte für $Z_{2}$ un 1/2 von 0 bis b, Stab 2/3 0 bis a, Stab 3/4 von bis h, Stab $4 / 0$ von 0 bis $\frac{1}{2}$ zu erstrecken. Man erhält

$\int M \frac{d M}{d Z_{1}}=Z_{1}\left[\frac{b^{3}+h^{3}}{3}+b^{2} a+\frac{(h-b)^{2} 1}{6}\right]$ $-Z_{2}\left[\frac{b a^{2}}{2}+\frac{b a 1}{2}-\frac{a(h-b) 1}{6}\right]$ $+Z_{3}\left[\frac{h^{2}}{2}+\frac{(h-b) 1}{6}\right]$ $-\mathrm{W}\left[\begin{array}{c}\mathrm{h}^{3} \\ 6\end{array}+\frac{\mathrm{h}(\mathrm{h}-\mathrm{b}) 1}{12}\right]=0 \ldots(\mathrm{c}$

$\int M \frac{d M}{d Z_{2}}=-Z_{1}\left[\frac{b a^{2}}{2}+\frac{b a^{2}}{2}-\frac{a(h-b) 1}{6}\right]$ $+Z_{2}\left[\frac{2 a^{2} 1}{3}+\frac{a l^{2}}{4}+\frac{a^{3}}{3}\right]$ $+Z_{3}\left[\frac{\mathrm{al}}{6}\right]-\mathrm{w}\left[\frac{\mathrm{ah} 1}{12}\right]=0 \ldots$

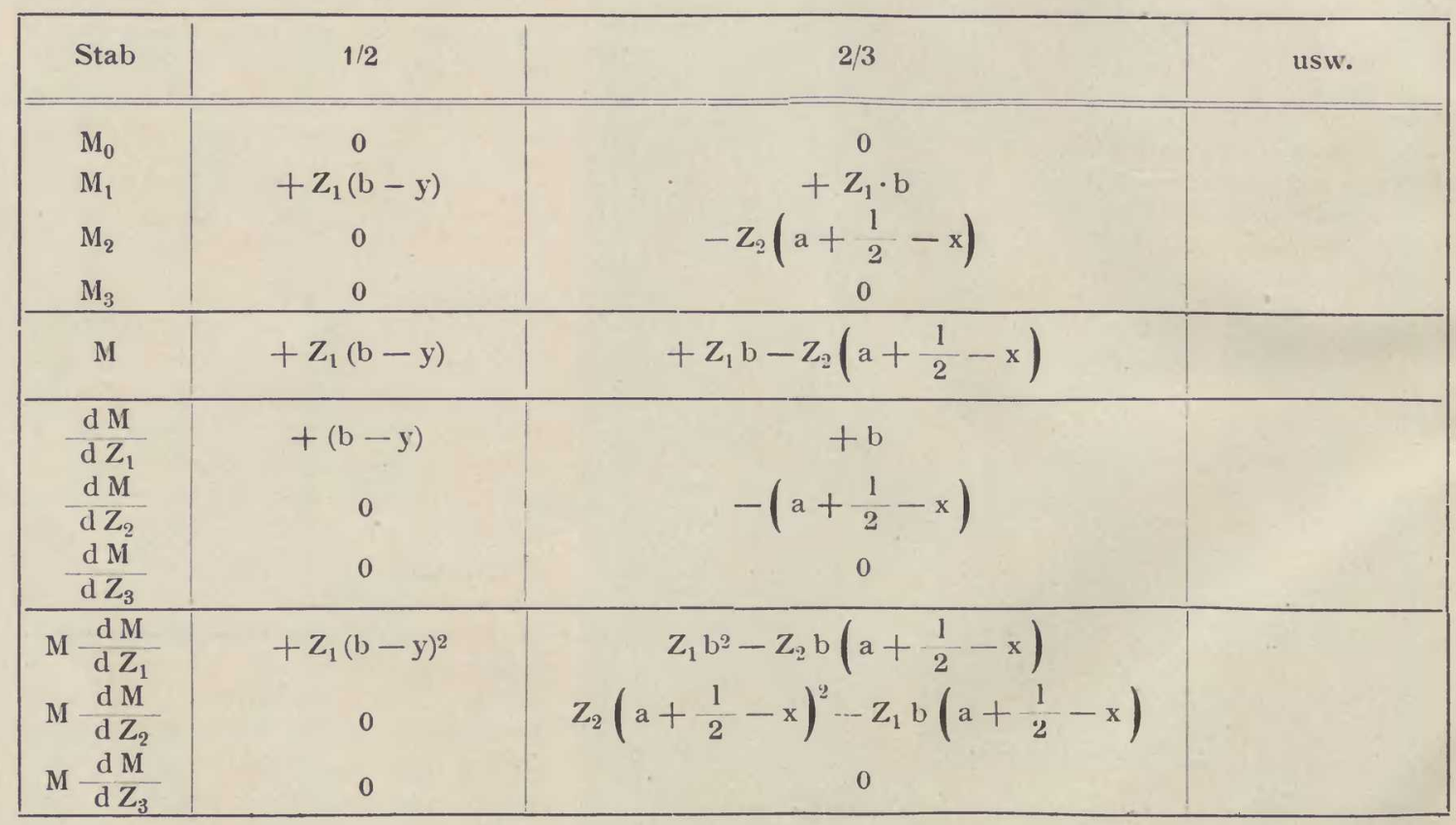

Es genügt, die Momente für eine Rahmen- $\int M \frac{d M}{d Z_{3}}=Z_{1}\left[\frac{h^{2}}{2}+\frac{(h-b) !}{6}\right]+Z_{2}\left[\frac{a 1}{6}\right]$ Halften einander gleich sind, jedoch das engegengesetzte Vorzeichen besitzen. werden. Die drei Arbeitsgleichungen sind in der Weise zusammenzustellen, daß die Werte

$+Z_{3}\left[h+\frac{1}{6}\right]-w\left[\frac{h^{2}}{4}+\frac{h 1}{12}\right]=0 \ldots$ Als Probe der Richtigkeit gilt, daßs die Bei-
werte $A_{12}$ und $A_{21}$ usw. einander gleich sind.
33

Nach Einsetzen der Stablingen elilt man der auf Biegung beanspruchten Querschnitte $\begin{array}{rll}\text { 2) }-133,368 Z_{1}+355,302 Z_{2}+7,205 Z_{3}-32,423 W & =0, \quad \text { nach den üblichen Dimensionierungstabellen be } \\ \text { 3) } 46,056 Z_{1}+7,205 Z_{2}+10,096 Z_{3}-25,181 \mathrm{~W}=0 . & \text { stimmt. Die Bewehrung ist aus Fig. } 21 \text { zu er- }\end{array}$ Man erhält

$Z_{1}=0,370 \mathrm{~W}$ in $\mathrm{t}$, $\mathrm{Z}_{2}=0,217 \mathrm{~W}$ in $\mathrm{t}$,
$Z_{3}=0,650 \mathrm{~W}$ in $\mathrm{mt}$,

$\mathrm{Z}_{3}=0,650 \mathrm{~W}$ in $\mathrm{mt}$,

und mit $W=55,85 \mathrm{t}$
Winddruck für eine Kuppelhälfte

$Z_{1}=20,85 \mathrm{t}$,

$Z_{2}=12,30 \mathrm{t}$
$Z_{3}=35,50 \mathrm{mt}$

(1)

$\begin{aligned} \text { Aus. diesen Werten } & \\ \text { assen sich alle Momente } & \end{aligned}$

und Auflagerkräfte er-

mitteln, sie sind in

Fig. 27 eingetragen.

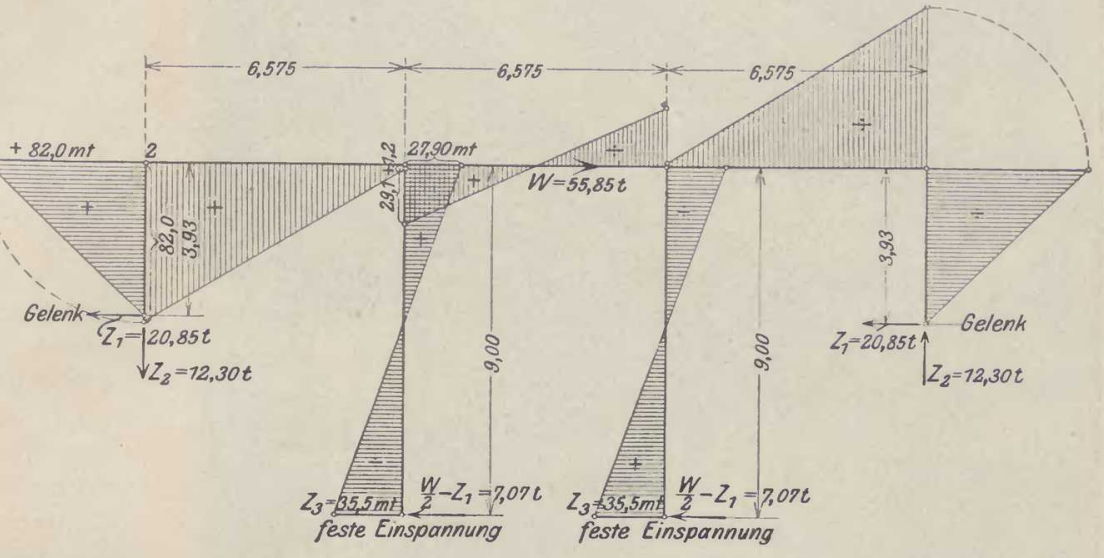

2. Einwirkung des

Eigengewichtes.

Der Riegel wird mit belastet. Wenn man, was angängig ist, die Einspannungdes Riegels an den Mittelstutzen vernachlasssigt, so wird der Rahmen ein Zweigelenk-
tahmen mit beweglicher Lagerung auf 2 Mittelstützen. Mit genügender Annäherung kann man das System unter Aut als durchgehenden $\mathrm{Bal}-$ ken auf 6 Stützen auffassen, der in den drei lastet ist. Man erhält dann die in Fig. 28 gezeichneten Eigengewichtsmomente. Die Summe der . ist in Fig. 29 dargestellt, die Pfosten erhalten außerdem noch die Kuppellasten in Höhe
von 200 t. Unter Vernachlässigung der Eisen
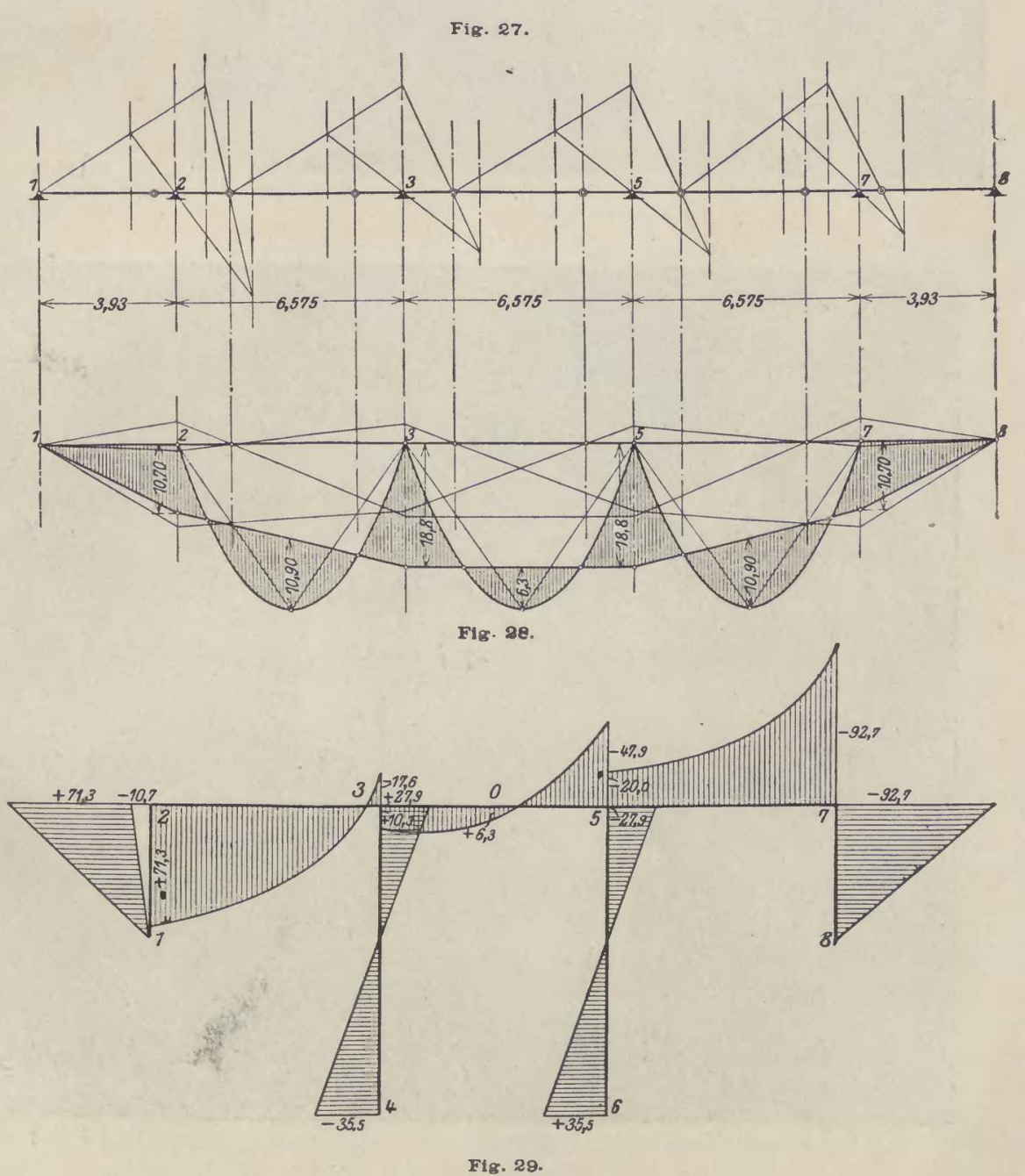
$-34-$

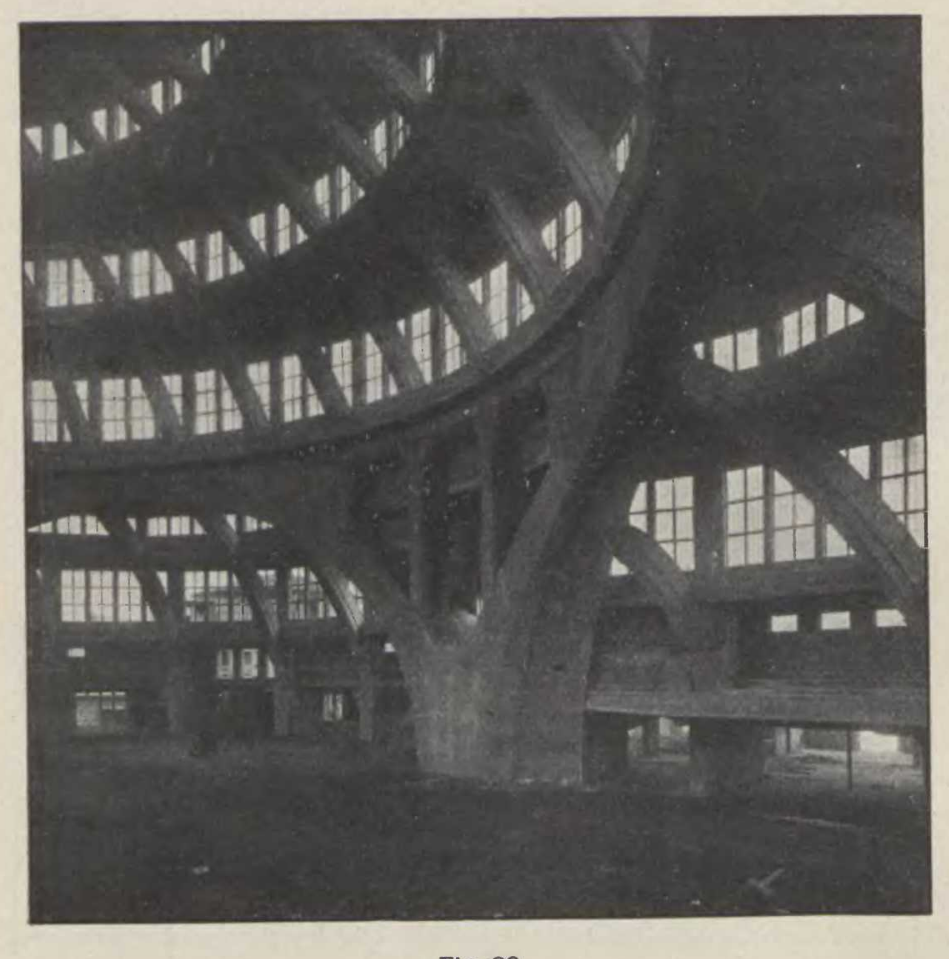

ehen. Auch die Mittelptosten sind, wiewohl sie infolge der großen Kuppellasten nur Druck erhalten, Beachtung wurde einer guten Ausmet. Fig. 30 gibt die doppelt krümmten Hauptbogen und den Windrahmen über dem Eckpfeiler wieder. Die Pfosten haben Aushalten. Wie der Rahmen architetonisch ausgestaltet werden soll, hängt im wesentlichen von der Akustik der Halle ab. Die Felder weder mit Stoff bespannt oder mit einer Eisenbetonwand geschlossen werden. In dem jetzigen unfertigen Zustande kommt gut die statische Geltung die auch bei etwas zurücktretender Wandfüllung noch erhalten bleiben würde.

$$
\text { Kuppel. }
$$

Fis. 30

Die Kuppel ist, wie eingangs
bereits kurz erwähnt, eine Rippen-

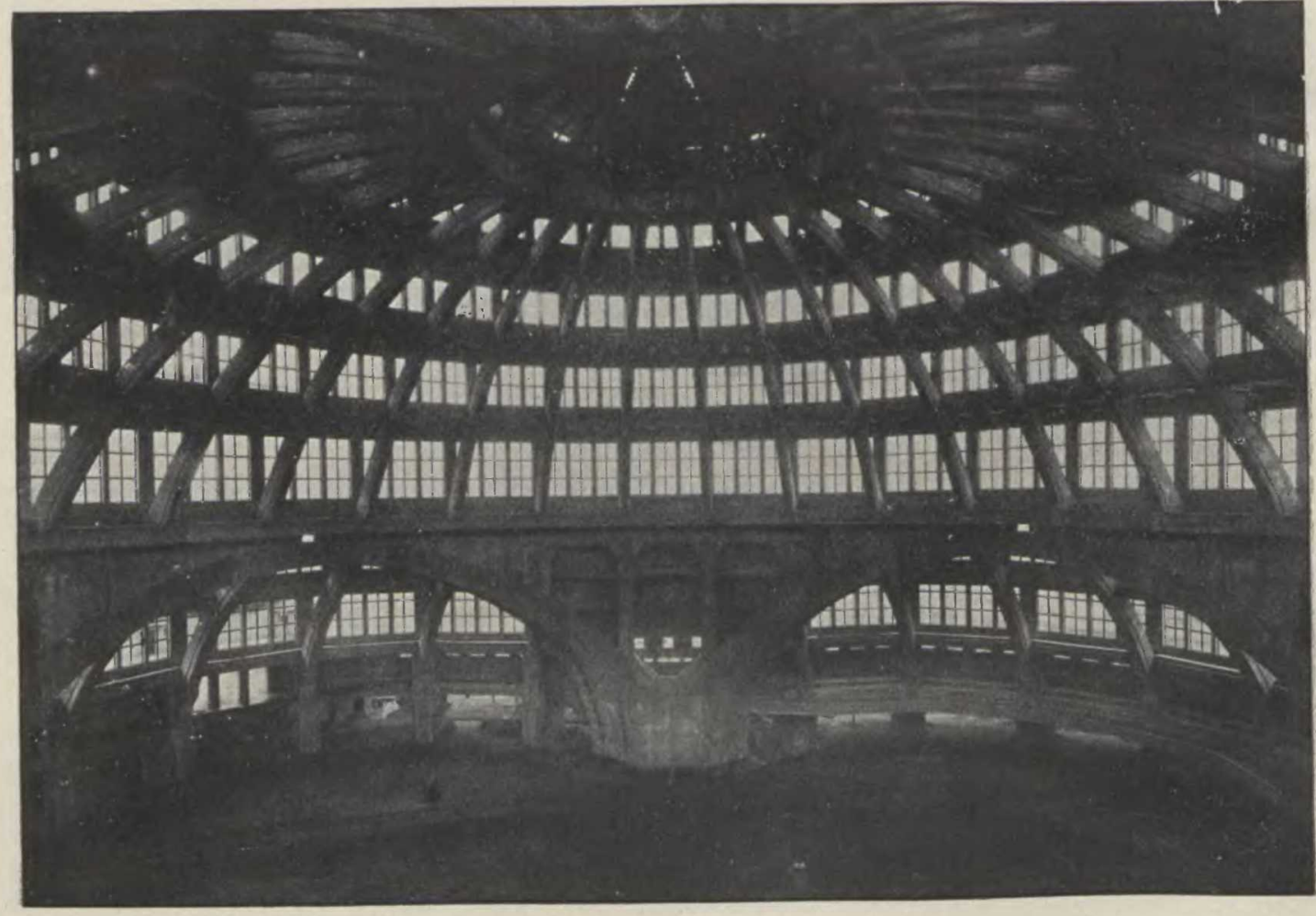

FIR. 31.
35

kuppel. Sie besteht aus 32 halben Bindern, die 1. Allgemeine Wirkung des Tragwerkes. Weite, obe 1. W. stützen, die Pfeilhöhe beträgt $161 \mathrm{~m}$, Der fachen Form bereits fur gleichmásig verteilte Druckring wird durch eine $5,75 \mathrm{~m}$ hohe Laterne heit ein verhältnismäßis schwierses Gebilde dr. aus 4 sich kreuzenden Bindern ausgesteift, während Der untere Zugring bietet zwar infolge der in die Kuppeibinder dur drei an die Ringdecken Verhaltnis zum Kuppeldurchmesser geringen Querden Binder herabgezogene, Versteifung tip in schnitsabmessun-

gegen seitliches Ausknicken gesichert werden der bewerliche

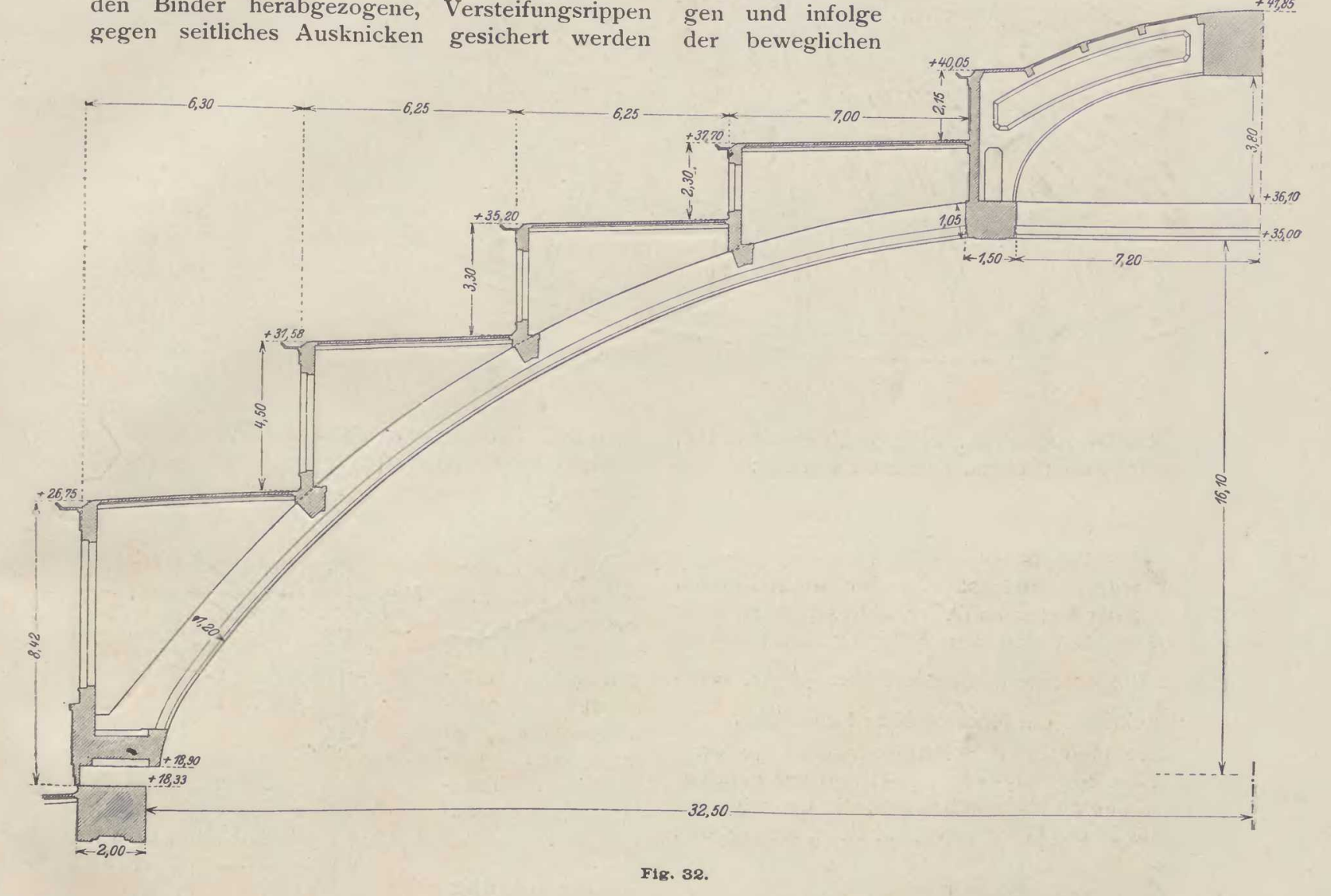

(s. Fig. 2 u. 3 und Fig. 32 u. 33). Unter den Lagerung des Zugringes einem Verdrehen der Kippvorrichtung angeordnet. Da die Streb on die Kuppelbinder aussteifenden Versteifungsden ziemlich hoch an dem Hauntbon und da ihre oberen Kämpferkräfte infolgedessen beiden berücksichtigt worden, da der Einfluß der nicht die Bogenachse treffen, würden beträchtliche oberen beiden erheblich zurtchtritt. Im GegenTorsion Um diesen Hauptbogen hierdurch auf- Widerstand des Druckringes gegen Verdrehung Kuppellager $0,18 \mathrm{~m}$ außerhalb der Mittellinie der berücksichtigt werden, um so mehr, als dieser durch Hauptbogen, so daß der Auflagerdruck mit der die sehr krattigen Laternenbinder wirksam aussultante bildet (s. Fig. 22).

Die Kuppel besitzt somit 67,36 m Stützweit und ist mit diesen Abmessungen zurzeit be

gesteift wird. durch ein wagerechtes Stuck Kunpelbinder erst nun gedacht, dessen Trägheitsmoment so groß ist, daß die Verbiegung dieses Binderstückes bei ver-
schiedenen äußeren Kräften der Verdrehung des 


\section{$-36-$}

Druckringes samt Laternenbindern gleich ist. Die

Die Verlängerung der mittleren Faser ist Abmessungen dieses gedachten Ersatzstückes,

$=\frac{\sigma_{0} 1}{\mathrm{E}}=\frac{1,68 \cdot 156}{14000}=0,00187 \mathrm{~cm}$

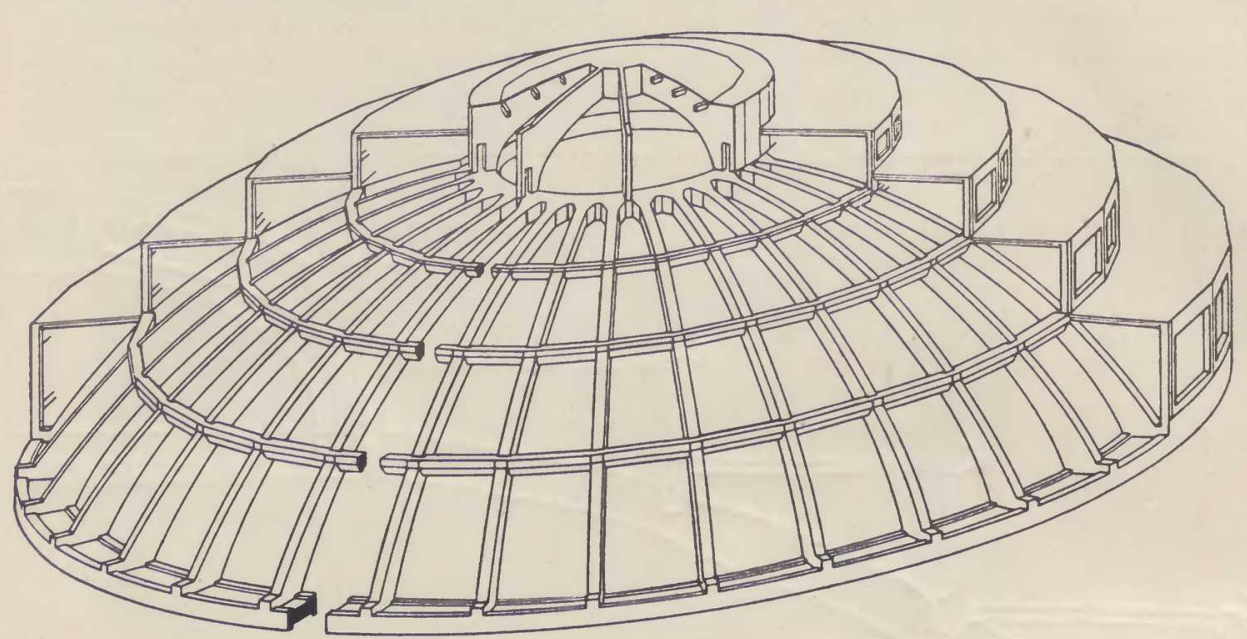

die radiale Ver$d_{\mathrm{r}}=\mathrm{d}_{1} \frac{\mathrm{r}}{1}$

$=\frac{0,00187 \cdot 795}{155}$

$=0,00955 \mathrm{~cm}$,

der Verdrehungs-
winkel

$\mathrm{s}_{\mathrm{d}}=\frac{2 \mathrm{~d} \mathrm{r}}{\mathrm{d}}$

$=\frac{2 \cdot 0,00955}{110}$

$=0,0001733$.

B) Druckring binder. F18. 33.

Kuppelbinder zu einem Zweigelenkbogen werd sind in folgender Weise bestimmt worden:

\section{a) Gleichmässig verteilte Lasten.}

Druckring ohne Laternenbinde

Es wurde zunáchst von der aussteifenden

Fall betrachtet, daß am Kämpfer sämtlicher 32

halben Binder eine Bogenkraft $H=\frac{1}{16,1} t$, also

Druckinge ein Moment $\mathrm{M}=1$ tm

Führt man in der Mitte zwischen je zwei selbst Momente $M_{r}$ anzubringen, die dem äußere nente $M$ das Gleichgewicht halten, sie ergebe sich $\mathrm{zu}$

$$
\mathrm{M}_{\mathrm{r}}=\mathrm{M} \frac{\mathrm{r}}{\frac{2 \mathrm{r} \pi}{32}}=5,1 \mathrm{M}
$$

Das Moment $\mathrm{M}_{\mathrm{r}}$ muß gleich sein der Summe $\sigma$ in dem Querschnitte $t$, also

$$
\int_{0}^{b} \int_{0}^{d} \sigma d f \cdot x=M_{r}
$$

$$
2 \frac{\sigma_{0} \mathrm{~d}}{4} \cdot \frac{\mathrm{d}}{3} \cdot \mathrm{b}=\mathrm{M}_{\mathrm{r}}
$$$$
\sigma_{\mathrm{n}}=\frac{6 \mathrm{M} \cdot 5,1}{\mathrm{~d}^{2} \mathrm{~b}}
$$

Setzt man die Zahlenwerte ein, so erhält man $\sigma_{0}=\frac{6 \cdot 1000 \cdot 100 \cdot 5,1}{110 \cdot 110 \cdot 150}=1,68 \mathrm{~kg} / \mathrm{qcm}$. teirende Wirkung der Laternenbinder wird die

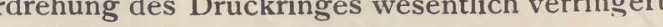
Da zwischen den 2 halben KuppelLaternenbinder ehen, hat $\operatorname{man} \mathrm{M}$ auf ieden Kuppel-
binder $1 / 4$ Laterinen Laterner$\begin{array}{ll}\text { binder von } 1 / 4 \text { des } \\ \text { atsächlich } & \text { vor- }\end{array}$ handenen Trägheitsmomentes zu-
rechnen. Untersuchung des Laternen-
binders allein Es werde zuVerdrehbarkeit des Druckringe also der WiderLaternenbinder, abgesehen, d. $h$. es werden $\mathrm{zu}$ nächst starre
Widerlager angenommen. Der Laternenbinder ist also dreifach sta-
tisch unbestimmt.
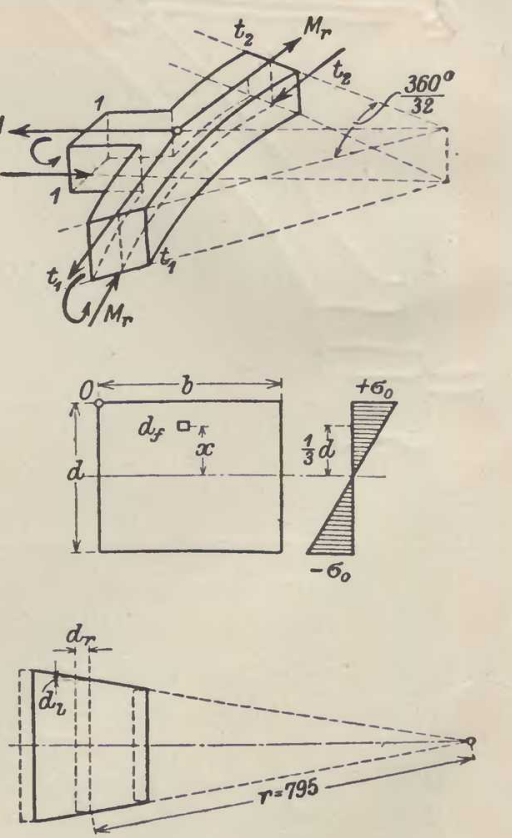

$\underset{2}{d}$
F18. 34.
Man führt nun in üblicher Weise in der Mitte enen Schnitt und bringt die drei Unbekannten. 政

Man erhalt den Angriffspunkt von $X_{c}$ in 3,60 er der Kämpferlinie und die Werte

$\frac{1}{2} \delta_{\mathrm{aa}}=-\frac{\varphi_{\mathrm{i}}}{\mathrm{s}_{\mathrm{i}}}=+\frac{0,223}{5270}=0,0000423$ $\frac{1}{2} \delta_{\mathrm{cc}}=\frac{0,266}{2} \mathrm{~mm}=0,000133 \mathrm{~m}$,

für $\frac{\mathrm{J}}{4}$ sind diese Werte zu vervierfachen, also wird

$$
\begin{aligned}
& \frac{1}{2} d^{\prime \prime}{ }_{\mathrm{aa}}=0,0001692 \\
& \frac{1}{2} d^{\prime \prime}{ }_{\mathrm{cc}}=0,000532 \mathrm{~m} .
\end{aligned}
$$

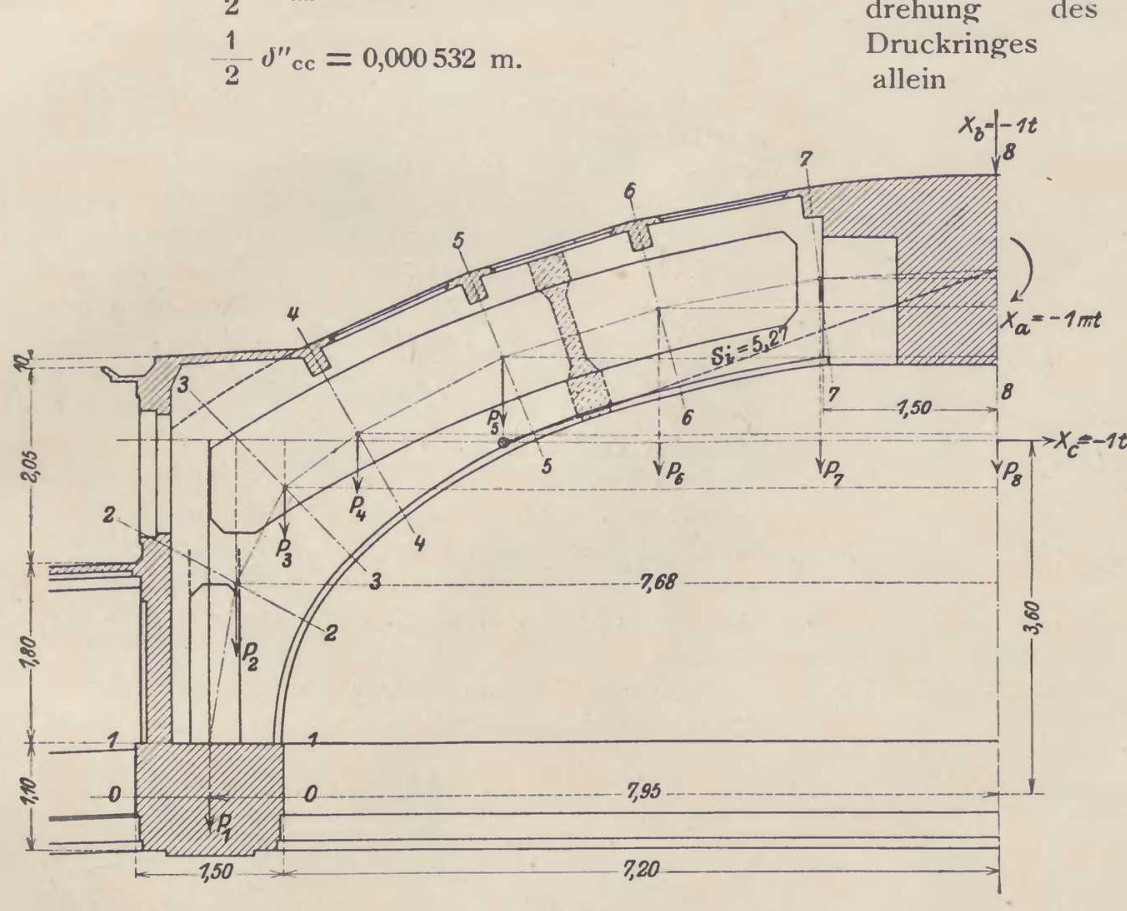

Untersuchung des Doppelgebildes. et zusammen also Hauptnetz.

introta. M $=1$ die folgenden Verdreh arseren erschiebungen (Fig. 36a). $\frac{1}{2} v^{\prime \prime}{ }_{\mathrm{a} m}=9^{\prime} \mathrm{d}=0,0001733$

regen die Lotrechte, der Punkt $C$ verschiebt sich um das Maf

$\frac{1}{2} v^{\prime} \mathrm{cm}=3,60 \mathrm{~g}^{\prime} \mathrm{d}=3,60 \cdot 0,0001733=0,000623 \mathrm{~m}$. $v_{\mathrm{aa}}=v^{\prime \prime}{ }_{\mathrm{aa}}+v^{\prime \prime} \mathrm{aa}=$

Zustand $X_{a}=1$. Das Moment $X_{a}=-1$ ruft hervor (Fig. 36b) 1. eine Verbiegung des Binders,
2. eine Verdrehung des Druckringes

Die Verdrehungen $\delta_{a}$ und die Verschiebunge Die Verdrehung Binders beträgt biegung allein $f^{\prime \prime}{ }_{\mathrm{az}}=0,0001692$ 2. durch die Verrehung
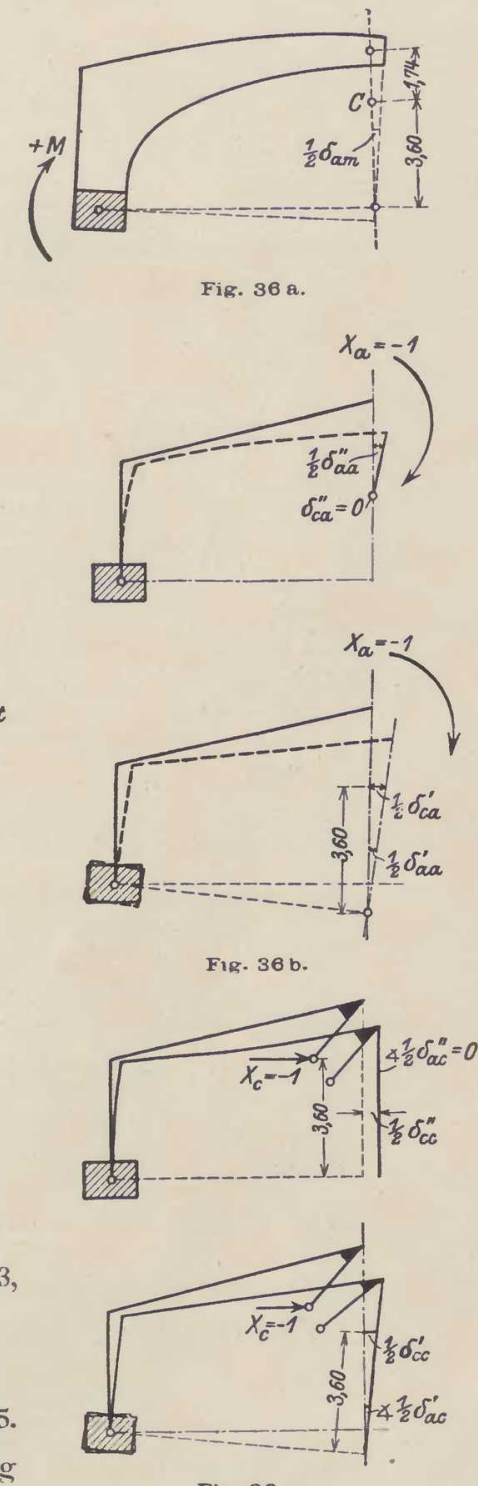
Die Verschiebun des Binderpunktes $\mathrm{C}$ be Fig. 38 c.

1. infolge der Verbiegung des Binders allei

2. infolge der Verdrehung des Druckringes allein $\frac{1}{2} f_{\mathrm{ca}}^{\prime}=0,0001733 \cdot 3,60=0,000623 \mathrm{~m}$, 
$38-$

zusammen also $\delta_{\mathrm{ca}}=2 \cdot 0,000623 \mathrm{~m}$

Zustand $\mathrm{X}_{\mathrm{c}}=-1$. In gleicher Weise ist für die Kraft $X_{c}=-$
die Verdrehung des Binders (Fig. 36c)

$\frac{1}{2} f^{\prime \prime \prime}{ }_{\mathrm{ac}}=0$,

$\frac{1}{2} J_{\mathrm{ac}}^{\prime}$

$=3,60 \cdot 0,0001733$

$=0,000623$

zusammen also

$J_{\mathrm{ac}}=2 \cdot 0,000623$,
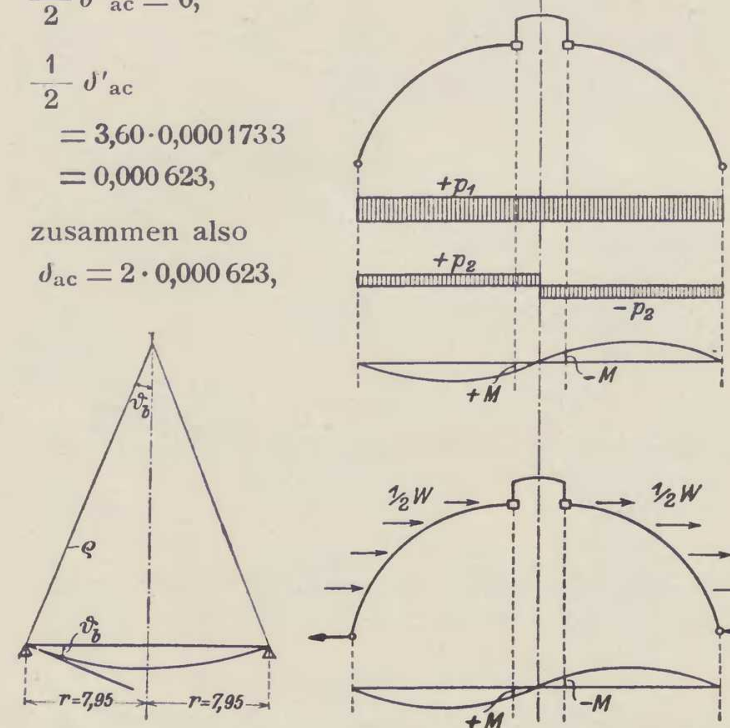

Fig. 37

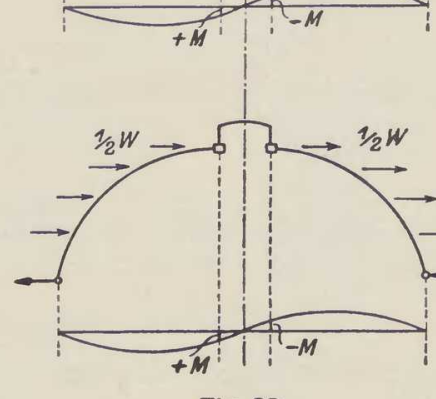

File. 38.

die Verschiebung des Punktes $\mathrm{C}$

$\frac{1}{2} J^{\prime \prime c c}=0,000532 \mathrm{~m}$,

$\frac{1}{2} f^{\prime \prime} \mathrm{cc}=3,60 \cdot 0,000623=0,002248 \mathrm{~m}$ zusammen also

$\delta_{c c}=2 \cdot 0,002780 \mathrm{~m}$.

Ermittelung von $X_{a}$ und $X_{e}$.

Es ist

$\delta_{\mathrm{ma}}-\mathrm{X}_{\mathrm{a}} \delta_{\mathrm{aa}}-\mathrm{X}_{\mathrm{c}} \delta_{\mathrm{ac}}=0$

$\mathrm{X}_{2}, 0,0003425+\mathrm{X}_{\mathrm{c}} 0,000623=0,0001733$

$\mathrm{X}_{\mathrm{a}} 0,000623+\mathrm{X}_{\mathrm{c}} 0,002780=0,000623$

$$
\mathrm{x}_{\mathrm{c}}=+0,187 \mathrm{t} \text {, }
$$

Die Verdrehung des Druckringes beträgt

$9_{\mathrm{d}} \mathrm{M}=0,0001733(1,000-0,166-0,187 \cdot 3,60)$ $=0,00002790$

Ersetzt man das Doppelgebilde, Druckring $m$ Balken (für jeden der 16 ganzen Binder) vom Querschnitte des anschließenden Binderstückes, $0,65 / 1,05 \mathrm{~m}$, mit dem Trägheitsmoment $\mathrm{J}=0,0627$
so ergibt sich für das Moment $\mathrm{M}=1 \mathrm{mt}$ mit

$$
\varrho=\frac{E J}{M}=87800 \mathrm{~m}
$$

für die Neigung der Tangente der Wert

$$
\sin \vartheta_{b}=9_{b}=\frac{r}{e}=\frac{7,95}{87800}=0,0000905 .
$$

Der Druckring mit Laternenbindern ist also ck vom Trägheits$\mathrm{J}^{\prime}=\mathrm{J} \frac{0,0000905}{0,00002790}=\mathrm{J} \cdot 3,24$,

d. h. vom etwa 3 fachen des anschließenden Kuppeld. h. vom etwa 3
binderstuckes.

b) UngleichmäBig verteilte Lasten

Eine ungleichmäßige Belastung je zweie gegenuber liegenden Binderhalften kann man sic mittlere Belastung und positive Z Zusatzlasten Seite. Die gleichmäßig verteilte Last ruft an Anschlusse an den Druckring auf der linken wie auf der rechten Binderhalte gleich grołse, gleic außen) hervor, die ungleichmäßige Belastung gegen gleich groise, aber entgegengesetzt wikende Momente. Die gleiche Wirkung ergib Winddruck, insoforn als durch die aussteifende Luv- und auf der Leeseite angreifend angeset werden kann (Fig. 38). (S. Berechnung auf Winddruck.) Betrachtet man zwei gegenüberliegende
Binderhälften, an denen links $+\mathrm{M}$, rechts -

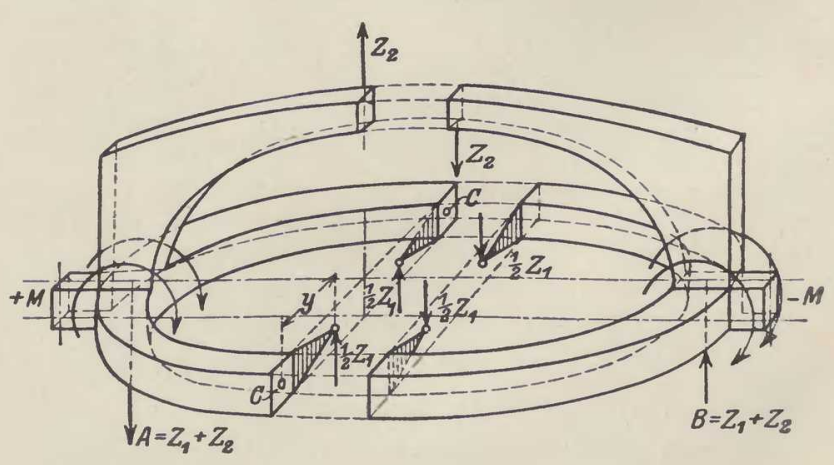$$
\text { Fig. } 38
$$

angreifen mögen, und schneidet man den DruckAchsenschnitt, so muß man zur Herstellung des Gleichgewichts außer den Auflagerkräften A un B im Schnitte Querkräfte $Z_{1}$ und $Z_{2}$ anbringen. Von diesen greift $Z_{2}$ im Laternenbinderschnitte
$-39-$

sondern an anschließenden starren Scheiben im Abstande y. Von der geringen Mitwirkung der unter $45^{\circ}$ zur Verbindungslinie A-B liegenden Rahmen und der diagonal stehenden Kuppelbinder
kann zur Vereinfachung abgesehen werden, sie entlasten sowohl den betrachteten Binder als de Druckring. Man kann je 4 Binder zusammenfassen und deren Wirkung mit dem Druckring
und je einem Laternenbinder betrachten (Fig. 39)

k) Druckring ohne Laternenbinder. Bei der Ermittelung der Verdrehung des $-\mathrm{M}$ muß man die Torsionswirkungen berücksichtigen, da man sonst völlig unzutreffende Ergebnisse erhä

Betrachtet man den Druckring allein, so müssen
Querkräfte $1 / 2 Z_{1}$ in solchem Querkafte $1_{2} Z_{1}$ Druckringachse angebracht wer-

$\begin{array}{ll} & \text { den, daß die Querkräte } 1 / 2 Z_{1} \text { im } \\ & \text { Verein mit den Momenten } 1 / 2 Z_{1}\end{array}$ Verdrehungen der geschni
yosschnitte $t$ verhindern

Die Verdrehung der Quera der Wirkung der Biegungs-

momente von $\mathrm{A}-\mathrm{C}$ und aus der $\mathrm{d}_{-\rightarrow 1} c$ auf dieser Strecke. Außerdem wird noch die senkrechte VerFig. 40. schiebung der Punkte $C$ gegen die Tangente in $A$ ermittelt. Es
werden getrennt die $Z$ ustände $1 / 2 Z_{1} \cdot y=Z \cdot y=Y$ und $1 / 2 Z_{1}=Z$ betrachtet.

$$
\text { Zustand } \mathrm{Y}=1
$$

Ein Moment $Y=1 \mathrm{mt}$ erzeugt in einem Querschnitte $\alpha$ ein Biegungsmoment $\mathrm{M}_{\mathrm{by}}=+1 \cos$

Biegungsmoment.

Die Verdrehung des Querschnittes $t-t$ infolge des Biegungsmomentes $y$ in der Richtung des Momentes $\mathrm{y}$ is

$$
\begin{aligned}
d^{\prime} y y & =\frac{1}{\mathrm{EJ}} \int_{0}^{\pi / 2} \mathrm{M}_{\mathrm{by}} \mathrm{M}_{\mathrm{by}} \mathrm{ds} \\
& =\frac{1}{\mathrm{EJ} J} \int_{0}^{\pi / 2} \cos ^{2} \alpha \mathrm{rd} \alpha \\
d_{y y}^{\prime y} & =\frac{\mathrm{r}}{4 \mathrm{EJ}}
\end{aligned}
$$

Die Verschiebung des Querschnittes $t-t$ infolge des Biegungsmomentes $Y$ in der Richtung der Kraft $Z$ ist mit $M_{b z}=+r \cos a$

$$
\begin{aligned}
v_{z y}^{\prime \prime} & =\frac{1}{\mathrm{E} \mathrm{J}} \int_{0}^{\pi / 2} \mathrm{M}_{\mathrm{bz}} \mathrm{M}_{\mathrm{by}} \mathrm{d} \mathrm{s} \\
& =\frac{1}{\mathrm{EJ}} \int_{0}^{\pi / 2} \mathrm{r} \cos \alpha \cos \alpha \mathrm{rd} \alpha \\
v_{z y}^{\prime \prime} & =\frac{\mathrm{r}^{2} \pi}{4 \mathrm{EJ}}
\end{aligned}
$$

Drehmoment.

Die Verdrehung zweier um die Längeneinheit Deh entfernten Querschnitte durch ein $\vartheta=3,6 \frac{\mathrm{b}^{2}+\mathrm{h}^{2}}{\mathrm{~b}^{3} \mathrm{~h}^{3}} \cdot \frac{\mathrm{M} \cdot}{\mathrm{G}}$

worin $\mathrm{G}=55000 \mathrm{~kg} / \mathrm{qcm}$ das Gleitmafs des Setzt

$$
\begin{aligned}
& \text { owird } \quad 3,6 \frac{\mathrm{b}^{2}+\mathrm{h}^{2}}{\mathrm{~b}^{3} \mathrm{~h}^{3}}=\frac{1}{\mathrm{~J}^{\prime \prime}} \\
& \vartheta=\frac{\mathrm{M} \vartheta}{\mathrm{J}^{\prime \prime} \mathrm{G}}
\end{aligned}
$$

nd die Summe der Verdrehungen

$$
s^{\prime \prime}=\frac{1}{\mathrm{~J}^{\prime \prime} \mathrm{G}} \int \mathrm{M} s \mathrm{~d} \mathbf{s}
$$

Für $\mathrm{Y}=1 \mathrm{mt}$ wird die Verdrehung des Querchnittes $t-t$ durch $Y$ in der Richtung von $Y$

$$
\begin{aligned}
J^{\prime \prime} y_{y} & =\frac{1}{J^{\prime \prime} G} \int_{0}^{\pi / 2} M_{9 y} M_{9 y} d s \\
& =\frac{1}{J^{\prime \prime} G} \int_{0}^{\pi / 2} \sin ^{2} \alpha r d \alpha
\end{aligned}
$$

\section{$f^{\prime \prime \prime}=\frac{\mathrm{r} \pi}{4 \mathrm{~J}^{\prime \prime} \mathrm{G}}$.}

Die Verschiebung in der Richtung $+\mathrm{Z}$ durch ird mit $M=1 \mathrm{r}(1-\sin \alpha)$

$$
\begin{aligned}
\delta^{\prime \prime}{ }_{z y} & =-\frac{1}{J^{\prime \prime} G} \int_{0}^{\pi / 2} M_{\vartheta_{z}} M \vartheta_{y} d s \\
& =-\frac{1}{J^{\prime \prime} G} \int_{0}^{\pi / 2} r(1-\sin \alpha) \sin \alpha r d \alpha
\end{aligned}
$$$$
\mathrm{d}^{\prime \prime}{ }_{\mathrm{zy}}=-\frac{\mathrm{r}^{2}}{\mathrm{~J}^{\prime \prime} \mathrm{G}}\left(1-\frac{\pi}{4}\right) .
$$ 
$-40-$

Zusammenfassun ist

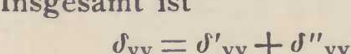

$=\frac{\mathrm{r} \pi}{4}\left(\frac{1}{\mathrm{EJ}}+\frac{1}{\mathrm{G} \mathrm{J}}\right)$

$\delta_{z y}=\delta^{\prime} z y+v^{\prime \prime}{ }_{z y}$

$=\frac{\mathrm{r}^{2} \pi}{4}\left(\frac{1}{\mathrm{EJ}}-\frac{1}{\mathrm{GJ}^{\prime \prime}}\left(\frac{4-\pi}{\pi}\right)\right)$.

Es ist bereits ermittelt

$\mathrm{M}_{\mathrm{bz}}=\mathrm{r} \cos \alpha$,
$\mathrm{M}_{9_{2}}=\mathrm{r}(1-\sin \alpha)$

Biegungsmoment. Die senkrechte Verschiebung des Quer-
schnittes $t-t$ durch $Z=1$ ist

$$
\begin{aligned}
J^{\prime} z z & =\frac{1}{E J} \int_{0}^{\pi / 2} \mathrm{M}_{\mathrm{bz}} \mathrm{M}_{\mathrm{bz}} \mathrm{ds} \\
& =\frac{\mathrm{r}^{3}}{\mathrm{EJ}} \int_{0}^{\pi / 2} \cos ^{2} \alpha \mathrm{d} \alpha
\end{aligned}
$$$$
v_{z z}^{\prime}=\frac{\mathrm{r}^{3} \pi}{4 \mathrm{E} \mathrm{J}} \text {. }
$$$$
\begin{gathered}
\text { Die senk } \\
\mathbf{Y}=1 \text { ist }
\end{gathered}
$$

$$
\frac{f_{y z}^{\prime}=f_{z y}^{\prime}=\frac{\mathrm{r}^{2} \pi}{4 E \mathrm{EJ}}}{\text { Drehmoment. }}
$$

Es ist die senkrechte Verschiebung infolge des Drehmomentes $\mathrm{M}_{\mathrm{y}}=-\mathrm{r}(1-\sin \mathrm{a})$

$$
\begin{aligned}
& f^{\prime \prime \prime}{ }_{z z}=\frac{1}{G J^{\prime \prime}} \int_{0}^{\pi / 2} \mathrm{M} g_{z} M 9_{z} \mathrm{ds} \\
& =\frac{1}{G J^{\prime \prime}} \int^{\pi / 2} r^{2}(1-\sin \omega)^{2} r d u \\
& J^{\prime \prime}{ }_{z z}=\frac{\mathrm{r}^{3}}{\mathrm{G} \mathrm{J}^{\prime \prime}} \cdot \frac{3 \pi-8}{4} \\
& \text { Die senkrechte Verschiebung durch ein Momen } \\
& \underline{J^{\prime \prime}{ }^{\prime}=J^{\prime \prime}{ }_{z y}=-\frac{\mathrm{r}^{2}}{\mathrm{G} \mathrm{J}^{\prime \prime}} \cdot \frac{4-\pi}{4} .} \\
& \text { Zusammenfassung. }
\end{aligned}
$$

Es ist insgesamt

$v_{z z}=\delta^{\prime}{ }_{z z}+j^{\prime \prime \prime}{ }_{z z}$
Ermittelung von $Y$.

Gesucht ist jetzt das Moment $\mathrm{Y}$, welches die Verdrehung des Querschnittes $t-t$ infolge $Z=t$ rückgängig macht. Man hat

$$
\begin{aligned}
& \mathrm{Y}=-\frac{\delta_{\mathrm{yz}}}{d_{y y}}=-\frac{\mathrm{r}^{2} 74\left[\frac{1}{\mathrm{EJ}}-\frac{1}{\mathrm{G}} \mathrm{J}^{\prime \prime} \cdot\left(\frac{4-\pi}{\pi}\right)\right]}{4 \mathrm{r} \pi\left(\frac{1}{\mathrm{EJ}}+\frac{1}{\mathrm{G} \mathrm{I}^{\prime \prime}}\right)} \\
& \begin{aligned}
E & =1400000 \mathrm{t} / \mathrm{qm} \\
\mathrm{J} & =0,1663 \mathrm{~m}^{4} ;
\end{aligned} \\
& G=550000 \mathrm{t} / \mathrm{qm} \\
& \frac{1}{\mathrm{EJ}}=4,295 \cdot 10-6 \\
& \frac{1}{\mathrm{G} \mathrm{J} \mathrm{J}^{\prime \prime}}=5,050 \cdot 10-6 \text {; } \\
& \frac{4-\pi}{\pi}=0,2725 \text {. }
\end{aligned}
$$

Hiermit erhält man

$$
Y=-r \frac{4,295-5,050 \cdot 0,2725}{4,295+5,050}
$$$$
\underline{Y=-r 0,312,}
$$

d. h. die Kraft $Z$ greift nicht im Punkte C, sondern in $\mathrm{C}^{\prime}$ im Abstande 0,312 $\mathrm{r}$ nach der Mitte zu an (Fig. 39).

ß) Druckring mit Laternenbinder

Die Verschiebung des Punktes $Z$ durch eine Kraft 1 im Punkte $C^{\prime}$ ist nun

$\Delta \mathrm{z}=\delta_{z \mathrm{z}}+\mathrm{Y} \delta_{\mathrm{zy}}$

$$
=\frac{\mathrm{r}^{3} \pi}{4} \cdot\left[\frac{1}{\mathrm{EJ}}+\frac{1}{\mathrm{GJ}^{\prime \prime}} \cdot\left(\frac{3 \pi-8}{\pi}\right)\right]
$$

$$
-\frac{0,312 \mathrm{rr}^{2} \pi}{4} \cdot\left[\frac{1}{\mathrm{EJ}}-\frac{1}{\mathrm{G} \mathrm{J}^{\prime \prime}} \cdot\left(\frac{4-\pi}{\pi}\right)\right]
$$

$=0,002240 \mathrm{~m}$.

Die Verschiebung des Punktes B gegen A ist $2 d z=0,004480 \mathrm{~m}$.

Für $\mathrm{M}=1$ ist $\mathrm{Z}=\frac{1}{2 r}$, also wird

$$
J_{\mathrm{mb}}=\frac{0,004480}{2 \cdot 7,95}=0,0002820 \mathrm{~m} \text {. }
$$

Denkt man sich jetzt lediglich noch den Laternenbinder durchschnitten und bringt an an, so ergeben diese in $A$ und $B$ Momente
$M=1 \mathrm{r}$, in $\mathrm{C}^{\prime}$ eine Querkraft $Z=1 / 2$, also die
$-41-$

benen ermittelte Verschiebung $\frac{2 \Delta \mathrm{Z}}{2}$ der Punkte $\mathrm{A}$ anschließ̧ende Binderstück ergeben. Dieser Ersatzwert ist ohne Bedeutung für die Untersuchung des gegen B. Dieser Verschiebung ist nun gleich die Kuppeltragwerkes punkte $\mathrm{m}, \mathrm{m}$ in der Richtung der Kraft $\mathrm{X}_{\mathrm{b}}$ infolge der Verdrehung des Druckringes allein. Hierzu kommt noch die Verschiebung dieVerbiegung der Rahnen die $d_{\mathrm{bb}}=2,690 \mathrm{~mm}=$

Insges eine Kraft $\mathrm{X}_{\mathrm{b}}=$

$d^{\prime} \mathrm{bb}=A \mathrm{z}+\delta_{\mathrm{bb}}=0,002240+$ $\begin{aligned} & 0,002690=0,004930 \mathrm{~m} \text {. } \\ & \text { Ein Moment } \mathrm{M}=1 \text { verschiebt }\end{aligned}$ $\underset{m}{\min }$ um

$J_{\mathrm{mb}}=0,0002820 \mathrm{~m}$

eine Kraft $\mathrm{X}_{\mathrm{b}}=-1 \mathrm{um}$ $\mathcal{N}^{\prime} \mathrm{x}=0,004930 \mathrm{~m}$
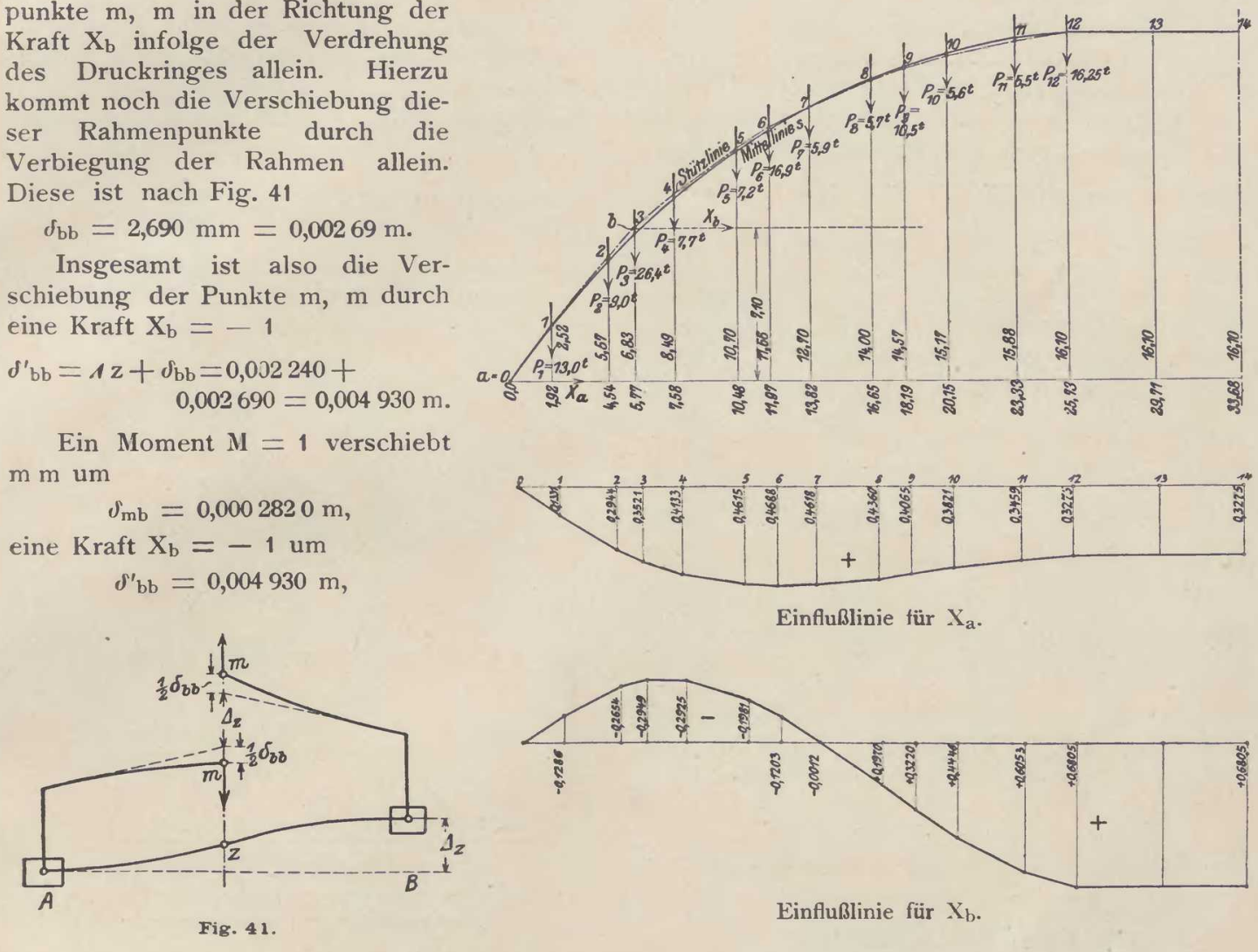

Einfußlinie tür $\mathrm{X}_{2}$

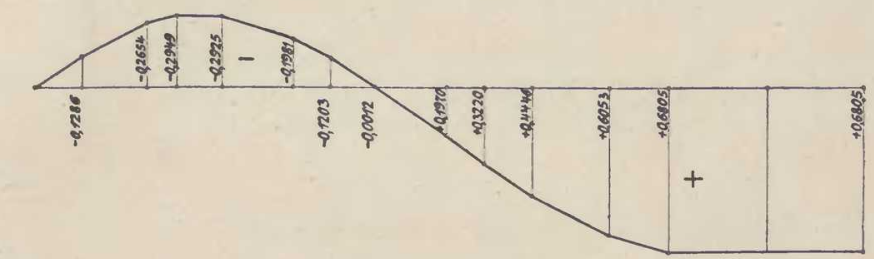

Einfluslinie für $\mathrm{X}_{\mathrm{b}}$

somit wird für $\mathrm{M}=1$ $\mathrm{X}_{\mathrm{b}}=\frac{0,0002820}{0,004930}=0,0572 \mathrm{t}$. Das Moment im Rahmenfufpunkt wird

$M_{d}=0,0572 \cdot 7,95=0,454$

Der Druckring hat also aufzu-

$\mathrm{M}_{\mathrm{d}}=1-0,454=0,546 \mathrm{mt}$ d. i. angenähert die Hälfte der Biegungsmomente.

Die senkrechte Verschiebung ${ }_{\mathrm{mb}}=0,546 \cdot 0,000282=0,0001539 \mathrm{~m}$.

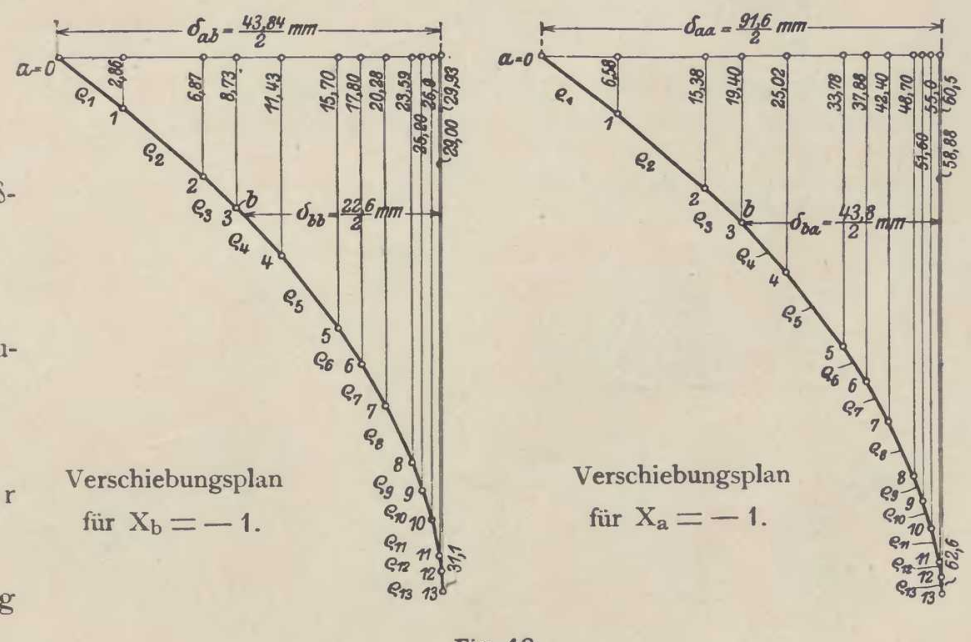
Wert ist lediglich die Kenntnis der Verteilung Die gleiche Verschiebung würde ein Binder- der
stuck von 1,67 fachem Trägheitsmomente wie das binder- 
$-42-$

2. Kuppelbinder und Zugring.

a) Bogenschübe $X_{a}$ und $X_{b}$.

Die Verschiebungspläne für den Zweigelenkbogen mit dem Ersatzmittelstück sind einmal Werte noch rechnerisch festgestellt worden wo bei die Bogenachse durch ein Vieleck ersetzt worden ist, in dessen Knichpunten die senk-$$
\text { chten Lasten angreifen (Fig. 42. }
$$

(c) Biegelinien für $X_{a}$ und $X_{b}$.

Die senkrechte Verschiebung $\delta_{\mathrm{ma}}$ eines Punktes rechten Verschiebung $\delta_{i m}$ des Punktes $O$ wage einer senkrechten Last $P_{m}=1$ in $m$, und zwar ist

$$
J_{\mathrm{ma}}=\int_{0}^{1} \frac{1}{E J} M_{\mathrm{a}} M_{\mathrm{m}} \mathrm{ds},
$$

erner ist

$$
\delta_{\mathrm{aa}}=\int_{0}^{1} \frac{1}{\mathrm{E} J} \mathrm{M}_{\mathrm{a}^{2}} \mathrm{~d} \mathbf{s} .
$$

Für symmetrische Belastung ist von $\mathrm{O}$ bis

Man hat nun zu bilden

$$
\frac{1}{E} \int_{0}^{1 / 2} \frac{M_{a} M_{m}}{J_{n}} d s=\frac{1}{E} \sum_{0}^{1 / 2} \frac{M_{a} M_{m}}{J_{n}} s_{m}
$$

für $\delta_{m a}$ erhält man

$$
\delta_{\mathrm{ma}}=\frac{1}{E} \sum_{0}^{m} y_{\mathrm{n}} x_{\mathrm{n}} x_{\mathrm{n}} s_{\mathrm{n}}+\frac{1 x_{\mathrm{m}}}{\mathrm{E}} \sum_{\mathrm{m}}^{1 / 2} \mathrm{~J}_{\mathrm{n}} s_{\mathrm{n}},
$$

für $\delta_{\text {aa }}$

$$
J_{\mathrm{aa}}=\frac{1}{\mathrm{E}} \sum_{0}^{1 / 2} \frac{\mathrm{y}_{\mathrm{n}}}{\mathrm{J}_{\mathrm{n}}} \mathrm{y}_{\mathrm{n}} \mathrm{s}_{\mathrm{n}}
$$

Man hat also drei Summenausdrücke

$$
\frac{\mathrm{y}_{\mathrm{n}}}{\mathrm{J}_{\mathrm{n}}} \mathrm{s}_{\mathrm{n}}, \quad \frac{\mathrm{y}_{\mathrm{n}}}{\mathrm{J}_{\mathrm{n}}} \mathrm{y}_{\mathrm{n}} \mathrm{s}_{\mathrm{n}}, \quad \frac{\mathrm{y}_{\mathrm{n}}}{\mathrm{J}_{\mathrm{n}}} \mathrm{x}_{\mathrm{n}} \mathrm{s}_{\mathrm{n}}
$$

zu bilden. Die Multiplikation der beiden Flächen $\mathrm{y}_{\mathrm{n}}$ mit $\mathrm{y}_{\mathrm{n}}$ oder $\mathrm{x}_{\mathrm{n}}$ ergibt (nach Müller-Breslau II. Bd., 2. Abt., S. 101) für eine Strecke $s_{n}$ (Fig. 43)

\section{$\int \mathrm{M}_{\mathrm{a}} \mathrm{M}_{\mathrm{m}} \mathrm{d}$}

$\frac{S_{n}}{6}\left[Z_{n}\left(2 Z_{n}^{\prime}+Z_{n+1}^{\prime}\right)+Z_{n+1}\left(2 Z_{n+1}^{\prime}+Z_{n}^{\prime}\right)\right]$ In ähnlicher Weise sind die Werte für $X_{b}$ Der Querschnitt des Hauptzugbandes ist in $\mathrm{F}=(260 \cdot 100+15 \cdot 617,2)=35250 \mathrm{qcm}$.

Für das gedachte gestreckte Zugband von Kämpzu Kämpfer ist ein Querschnitt anzusetzen $\mathrm{F}^{\prime}=\frac{1}{5,1} \cdot 35250=6920 \mathrm{qcm}$, somit beträgt die Verlängerung für $\mathrm{X}_{\mathrm{a}}=1$

$$
J_{z a}=\frac{1000 \cdot 6736}{140000 \cdot 6920}=0,0069 \mathrm{~cm} .
$$
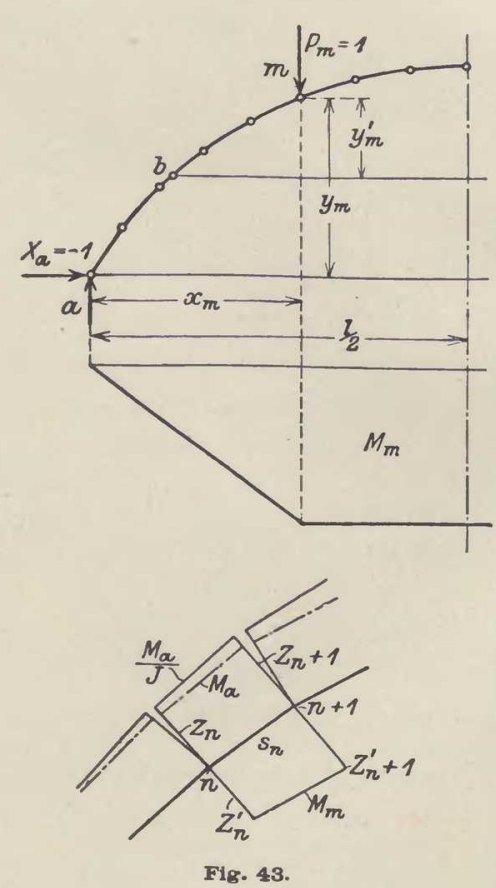
In gleicher Weise ist für das zweite Zug-
band mit

$$
\begin{aligned}
& F^{\prime}=\frac{1}{5,1} \cdot 6645=1300 \mathrm{qcm}, \\
& J_{z b}=\frac{1000 \cdot 5520}{140000 \cdot 1300}=0,0303 \mathrm{~cm} .
\end{aligned}
$$

$-43-$

ß) Ermittlung von $X_{a}$ und $X_{b}$ b) Eigengewicht.

Es empfiehlt sich nicht, die beiden Unbekannen $X_{a}$ und $X_{b}$ aus zwei Gleichungen mit naten für $\mathrm{X}_{\mathrm{a}}$ und $\mathrm{X}_{\mathrm{b}}$ (Fig. 42) und den EigenWewichislasten erhalt man die statisch unbestimnmit Doppelzeigern dadurch zu vermeiden, da

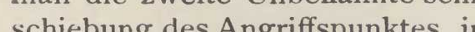
Unbekannten einfürt (siehe auch Nüler-Breshu II. Bd., 2. Abt. S. 156).

Auf rechnerischem Wege war gefunde

$J_{\mathrm{aa}}=9,148 \mathrm{~cm}, \quad \delta_{\mathrm{za}}=0,0069 ;$

$J_{\mathrm{ab}}=4,396 \mathrm{~cm}, \quad J_{\mathrm{zb}}=0,0303$;

$\mathrm{J}_{\mathrm{bb}}=2,255 \mathrm{~cm}$.

Es ist

$\operatorname{tg} a=\frac{J_{\mathrm{ab}}}{2\left(\delta_{\mathrm{aa}}+J_{\mathrm{za}}\right)}$

$\sin \alpha=0,23346$,

$\cos \alpha=0,97237$;

$\mathrm{X}_{\mathrm{a}}=\mathrm{X}_{\mathrm{a}}^{\prime}-\mathrm{X}_{\mathrm{q}} \sin \alpha=\frac{J_{\mathrm{ma}}}{\gamma_{\mathrm{aa}}+J_{\mathrm{za}}}-\mathrm{X}_{\mathrm{q}} \sin \alpha_{\mathrm{s}}$

$\mathrm{X}_{\mathrm{b}}=\mathrm{X}_{\mathrm{q}} \cos \boldsymbol{\mu}$,

$\mathrm{X}_{\mathrm{q}}=\frac{\frac{\delta_{\mathrm{mb}} \cos \alpha}{2}-\delta_{\mathrm{ma}} \sin \alpha}{\frac{2}{\frac{\left(\delta_{\mathrm{bb}}+\delta_{\mathrm{bz}}\right) \cos ^{2} \alpha}{4}-\frac{\delta_{\mathrm{ab}} \sin \alpha \cos \alpha}{2}}}$

$=\frac{s_{\mathrm{mq}}}{\mathrm{v}_{\mathrm{qq}}}$

$S_{\mathrm{qq}}=\frac{2.2853 \cdot 0,97237^{2}}{4}-\frac{4,396 \cdot 0,23346 \cdot 0,97237}{2}$

$=0,540190-0,498967=+\underline{0,041223 .}$.

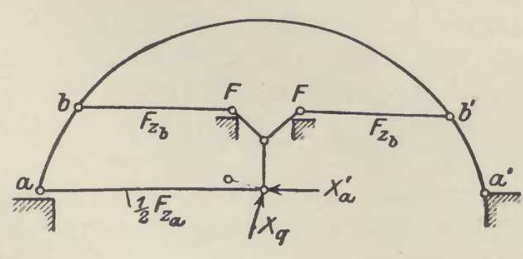

$\frac{1}{2} \delta_{a b} \sqrt{\delta_{\alpha a d}+\delta_{z a}}$ Fis. 44

\begin{tabular}{r|r|c|c|c|c}
\hline Punkt & Last $\mathrm{P}$ & $\mathrm{X}_{\mathrm{a}}$ & $\mathrm{PX}_{\mathrm{a}}$ & $\mathrm{X}_{\mathrm{b}}$ & $\mathrm{P}_{\mathrm{b}}$ \\
\hline \hline 1 & 13,0 & 0,1331 & 1,7303 & $-0,1286$ & $-1,6718$ \\
2 & 9,0 & 0,2944 & 2,6496 & $-0,2654$ & $-2,3886$ \\
3 & 25,4 & 0,3521 & 9,2954 & $-0,2949$ & $-7,7854$ \\
4 & 7,7 & 0,4133 & 3,1824 & $-0,2925$ & $-2,2523$ \\
5 & 7,2 & 0,4615 & 3,3228 & $-0,1981$ & $-1,4263$ \\
6 & 16,9 & 0,4688 & 7,9227 & $-0,1203$ & $-2,0331$ \\
7 & 5,9 & 0,4618 & 2,7246 & $-0,0012$ & $-0,0071$ \\
8 & 5,7 & 0,4360 & 2,4852 & $+0,1970$ & $+0,1229$ \\
9 & 10,5 & 0,4605 & 4,2683 & $+0,3220$ & $+3,3810$ \\
10 & 5,6 & 0,3821 & 2,1398 & $+0,4446$ & $+2,4898$ \\
11 & 5,5 & 0,3459 & 2,0862 & $+0,6053$ & $+2,9447$ \\
12 & 16,25 & 0,3275 & 5,3219 & $+0,6805$ & $+11,0581$ \\
\hline & 129,65 & & 47,1292 & & $-17,5646$ \\
& & & & & $+20,9965$ \\
& & & & &
\end{tabular}

Für beide Hälften erhält man somit:

$\mathrm{X}_{\mathrm{a}}=2 \cdot 47,1292=94,2584 \mathrm{t}$. $\mathrm{X}_{\mathrm{b}}=2(20,9965-17,5646)=6,8638 \mathrm{t}$.

Mit den Werten $M_{0}, X_{a}$ und $X_{b}$ ergeben sic die fol den $\mathrm{W}_{0}$, $\mathrm{M}=\mathrm{M}_{\mathrm{c}}-\mathrm{X}_{\mathrm{a}} \mathrm{y}-\mathrm{X}_{\mathrm{b}} \mathrm{y}^{\prime}$

\begin{tabular}{c|c||r|c}
\hline Punkt & $\mathrm{M}_{0}-\mathrm{X}_{\mathrm{a}} \mathrm{y}-\mathrm{X}_{\mathrm{b}} \mathrm{y}^{\prime}$ & Punkt & $\mathrm{M}_{0}-\mathrm{X}_{\mathrm{a}} \mathrm{y}-\mathrm{X}_{\mathrm{b}} \mathrm{y}^{\prime}$ \\
\hline \hline 1 & $+11,40$ & 7 & $+2,14$ \\
2 & $+29,53$ & 8 & $-6,07$ \\
3 & $+43,17$ & 9 & $-5,43$ \\
4 & $+24,24$ & 10 & $-12,48$ \\
5 & $+14,04$ & 11 & $-15,12$ \\
6 & $+15,83$ & 12 & $+1,63$
\end{tabular}

In Fig. 42 ist die Stuttzlinie für Eigengewich eingezeichnet. 
$-44-$

c. Winddruck.

bildete System sei das Hauptnetz. Die Kräfte $\mathrm{H}_{\mathrm{r}}$ Kuppelbinder durch die Ringdecken die genaue Berechnung äußerst schwierige Verhält- Kämpferpunkte aller übrigen mit $\mathrm{W} \cos \beta_{r}$ belastenisse. Da nun insbesondere jede Großkonstruktion ten Binder um $\boldsymbol{A}_{0}$ verschoben werden, also auf während der Ausführung leicht unberechenbaren einem um $\boldsymbol{d}_{0}$ gegen die Anfangslage verschobenen Einfüssen unterworfen ist, kam es auf an; die rechnungsmälsig auftretenden Spannungen peinlich genau $z$ ermitteln, als vielmehr darauf, für alle ave den Hauptraggliedern unbeding wehrungen zu geben. Daher sind für die einzelnen Teile jeweils möglichs ungunstige Annahmen gemacht wor den, die indessen gleichzeitig im ganDie große Steifigkeit der Ringdecken in ihrer Ebene gestattet die Annahme, daß sie den auf die zugehorigen Wande treftenden Windaruck Leeseite in die Binder Denn bei einer anderen Verteilun würden entsprechende Binderpunkte verschieden grolse selthene VerschieStarrheit der Ringdecken nicht möglich sind. Abgesehen von dieser be allen Decken vorhandenen kraftübertragenden Wirkung wurde angenomRingdecken auf die Kuppelbinder aussteifend wirken, die oberen nicht unmittelbar mit den Bindern verbundenen, etwas hoher anschneidenden dagege folgender Weise in den Unterbau ge-

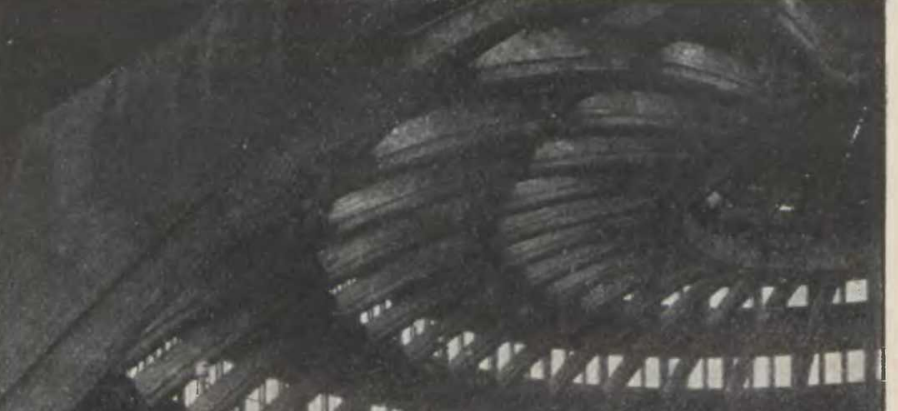

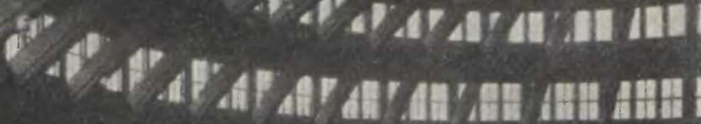

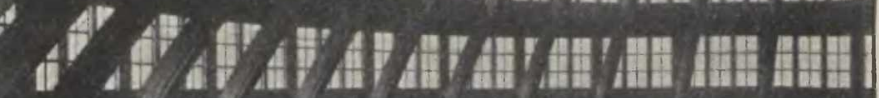
An Stelle des Druckringes mit den at steifenden Laternenrahmen wird für jeden der 16 eingeführt, die Binder werden also im Mittelpunkte fest miteinander verbunden gedacht (Fig. 46). Man löst nun die Verbindungen sämtlicher Binder mit
dem Zugbande bis auf Binder 0 , der fest verbunden bleibt. An allen anderen Schnittstellen sei die Verbindung nur insoweit vorhanden, dar lediglich die Bogenkrärte des Binders infolge des sie treffenden Winddruckes übertragen werden (wenn $\mathrm{W}=$ Winddruck auf den voll getroffenen Binder, $\beta_{r}=$ Neigungswinkel des Binders $r$ geoen die Windrichtung ist). Der Zugring überträgt dann in irgend einer Weise durch die Rollenlager
die Winddrücke $H_{x}$ in den Unterbau. Das so ge-

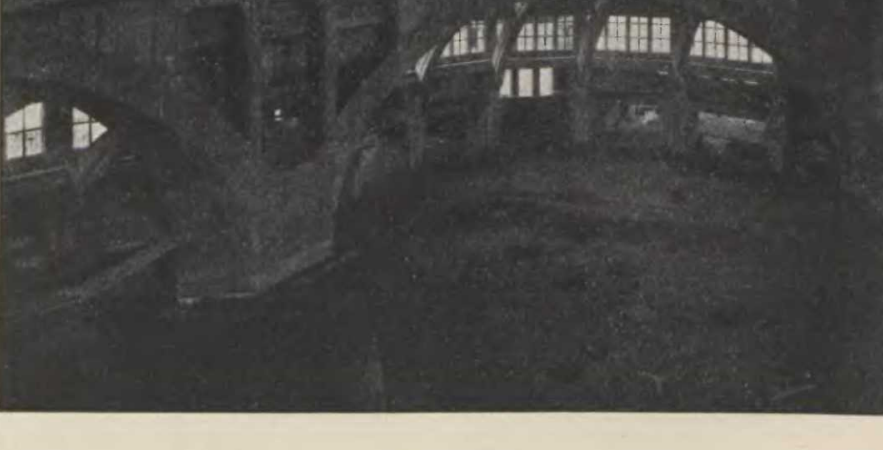

Fig. 45.

Kreise liegen, unabhängig von der Größe de

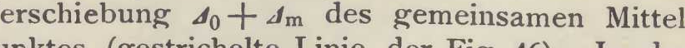

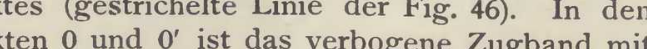
Kampern verbunden, in $1,2,3, \ldots$ ergeben

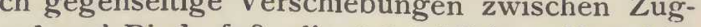

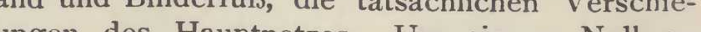
政 äfte $X_{1}, X_{2}, X_{3} \ldots$ wirken, das System ist lso 7 fach statisch unbestimmt, wenn man die oeingere Seitensteifigkeit der Binder unberucksichgebei der die Lager in der Windrichtung keine

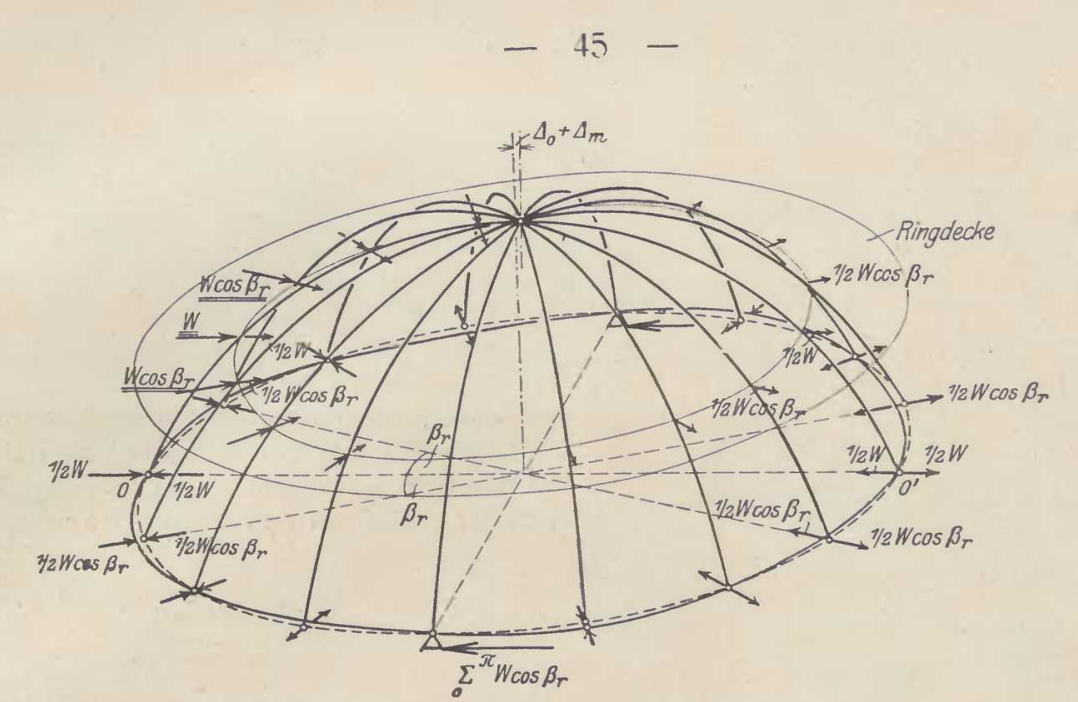

Zugring verbogen durch die Krä̈te $\frac{1}{2} W \cos \beta$

- - . Kreis, auf dem die Fig. 48.

Kraft übertragen. So kann man z. B. annehmen,
daß die Lagerdrücke mit dem Sinus des Nei dungswinkels zunehmen, d. h. daß der Neidruck jedes Binders durch die senkrecht zu ihm liegenden Lager übertragen wird (Fig. 47). Die Kräfte im Zugringe werden in folgender Weise

Trennt man den geschlossenen Ring, der von den 4 symmetrisch liegenden Lasten $P_{r}=1$ be-
lastet und in $A$ und $B$ wagerecht verschiebbar gelagert ist, in diesen Punkten, so hat man an während ein Moment nicht auftritt (Fig. 48a). Zur Bestimmung der Querkraft hat man die Verschiebungen für das Hauptnetz und für
$\mathrm{Y}=1 \mathrm{zu}$ bilden. Im Hauptnetze ist

$$
\delta_{\mathrm{ar}}=-\frac{1}{\mathrm{E} J} \int^{\pi / 2} \mathrm{M}_{\mathrm{a}} \mathrm{M}_{\mathrm{or}} \mathrm{ds},
$$

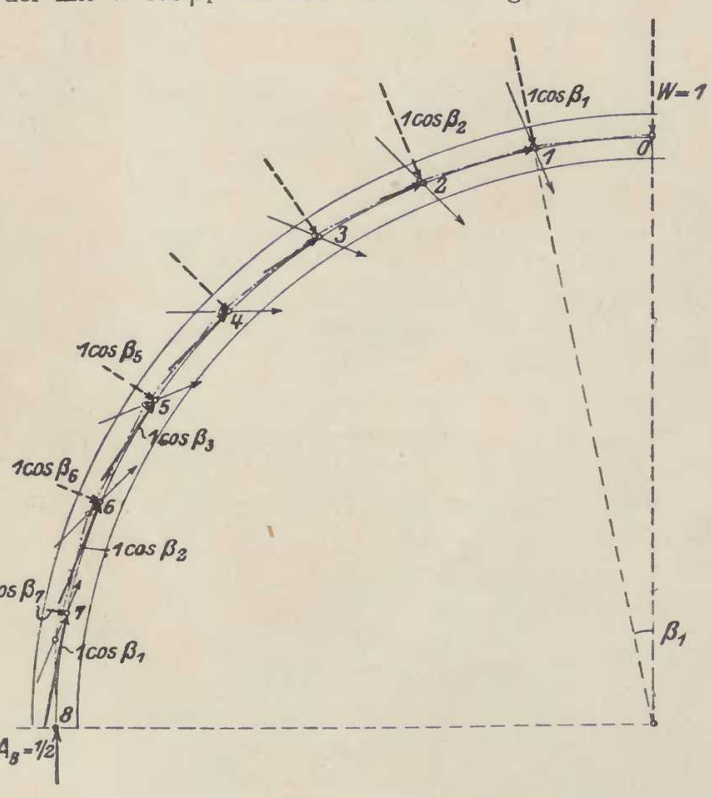

worin

$$
\begin{aligned}
& \mathrm{M}_{\mathrm{a}}=-1 \cdot \mathrm{y}=-\mathrm{r} \cos \beta_{\mathrm{x}}, \\
& \mathrm{M}_{\mathrm{or}} \text { zwischen A und } \mathrm{r} \\
& \mathrm{M}_{\mathrm{or}}=1 \cdot \cos \beta_{\mathrm{r}} \mathrm{x} \\
&=1 \cdot \cos \beta_{\mathrm{r}}\left(1-\sin \beta_{\mathrm{x}}\right), \\
& \text { zwischen } \mathrm{r} \text { und } \mathrm{C} \\
& \mathrm{M}_{\mathrm{or}}= 1 \cos \beta_{\mathrm{r}} \mathrm{x}-1 \mathrm{r} \sin \left(\beta_{\mathrm{r}}-\beta_{\mathrm{x}}\right) \\
&= \mathrm{r}\left[\cos \beta_{\mathrm{r}}\left(1-\sin \beta_{\mathrm{x}}\right)\right. \\
&\left.-\sin \left(\beta_{\mathrm{r}}-\beta_{\mathrm{x}}\right)\right] .
\end{aligned}
$$

Man erhält

$$
J_{\mathrm{ar}}=-\frac{\mathrm{r}^{3}}{2 \mathrm{EJ}}\left(\cos \beta_{\mathrm{r}}-\sin \beta_{\mathrm{r}} \cdot \boldsymbol{\beta}_{\mathrm{r}}\right) .
$$

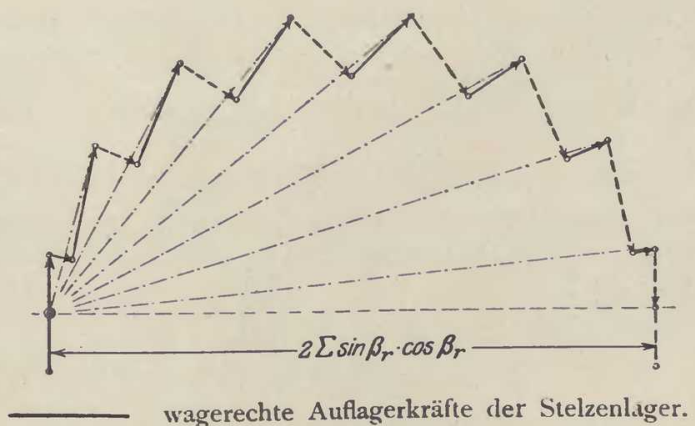

Kämpferkrä̆fte der Binder.

Fig. 47 


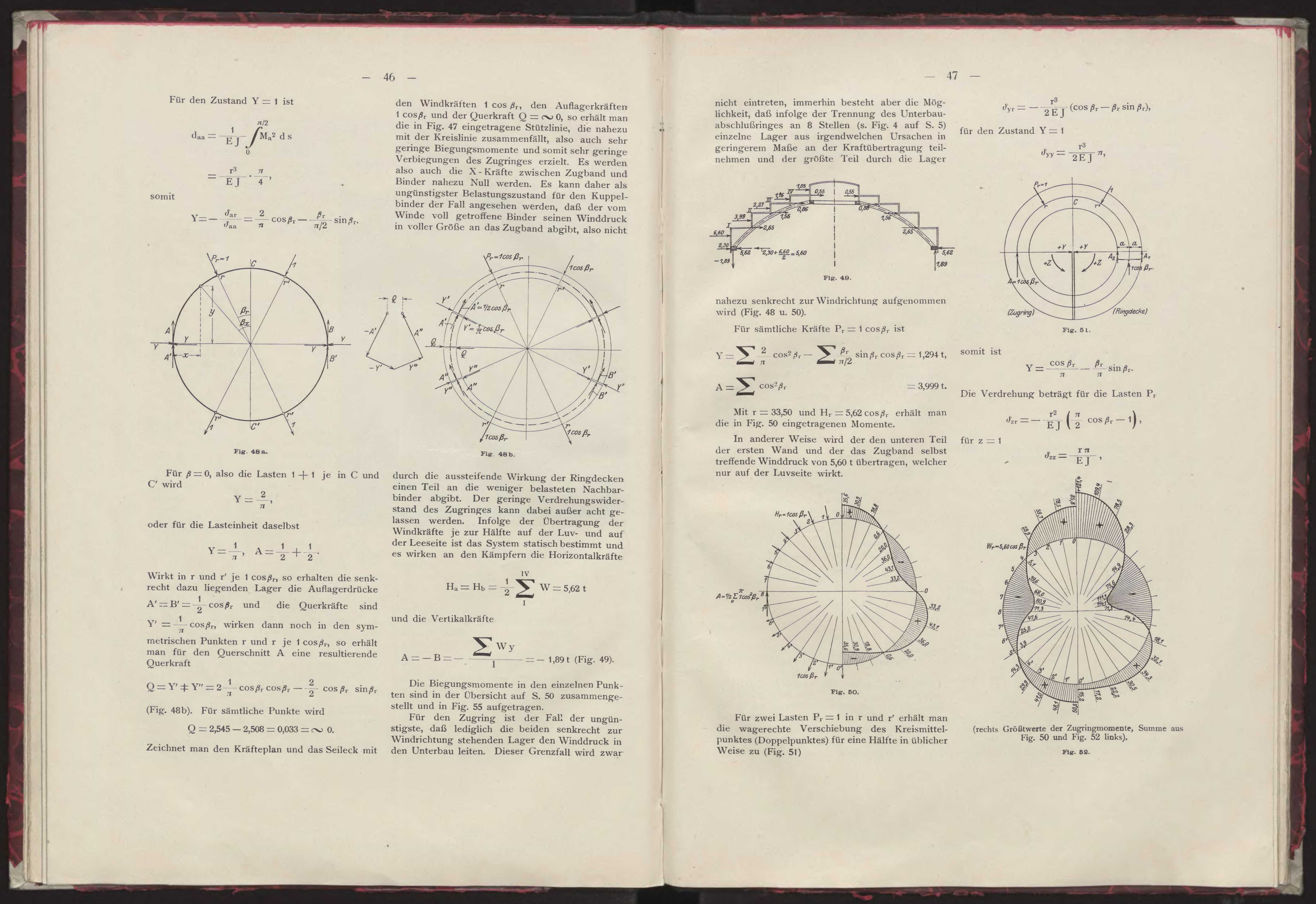


$-48-$

$$
Z=r\left(\frac{1}{2} \cos \beta_{r}-\frac{1}{\pi}\right)
$$

Für alle Kräfte $P_{r}=1 \cos \beta_{r}$ erhält man:

$\mathrm{Y}=\sum \frac{\cos ^{2} \beta_{\mathrm{r}}}{\pi}-\sum \frac{\beta_{\mathrm{r}}}{\pi} \sin \beta_{\mathrm{r}} \cos \beta_{\mathrm{r}}=+0,647$,

$Z=\sum r\left(\frac{1}{2} \cos ^{2} \beta_{r}-\frac{\cos \beta_{\mathrm{r}}}{\pi}\right)=+0,380 \mathrm{r}$

$\mathrm{A}=\sum \cos ^{2} \beta_{x}$

Mit $\mathrm{r}=33,50$ und $\mathrm{W}_{\mathrm{r}}=5,60 \cos \beta_{\mathrm{r}}$ erhält man die in Fig. 52 links eingetragenen Momente. Die eingetragen, der Größtwert ist demnach 121,4 mt. Die Zusatznormalkraft im Zugringe beträgt
für die Kräfte $1 \cos \beta_{\mathrm{r}}$ auf der Luv- und Leeseite

$$
\begin{aligned}
& \begin{aligned}
Z_{\mathrm{w}} & =\sum \mathrm{Y}+\sum \cos \beta_{\mathrm{r}} \sin \beta_{\mathrm{r}} \\
& = \pm 3,802 .
\end{aligned} \\
& \text { Mit } \mathrm{H}_{0}=5,62 \mathrm{t} \text { wird }
\end{aligned}
$$$$
Z_{\mathrm{w}}= \pm 21,5 \mathrm{t} \text { ( }+ \text { auf der Leeseite) }
$$

Für die lediglich auf der Luvseite angreifenden Kräfte $1 \cos \beta_{r}$ wird die Zusatznormalkraft auf der Luvseite

$$
\begin{aligned}
& \qquad \begin{aligned}
Z_{\mathrm{w}}=-\sum \mathrm{Y}- \\
=-3,155 .
\end{aligned} \\
& \text { Für } \mathrm{W}_{0}=5,60 \mathrm{t} \text { wird } \beta_{\mathrm{r}} \sin \beta_{\mathrm{r}} \\
& \qquad \begin{aligned}
\mathrm{Z}_{\mathrm{w}}=-17,7 \mathrm{t}, \\
\qquad \begin{aligned}
\mathrm{Z}_{\mathrm{w}} & = \\
& = \\
= & +0,647,
\end{aligned}
\end{aligned}
\end{aligned}
$$

auf der Leeseite

für $\mathrm{W}_{0}=5,60$

$$
Z_{w}^{\prime}=3,6 \mathrm{t} .
$$
Insgesamt ist auf der Luvseite $Z_{w}=-39,2 t$, auf
der Leeseite $+25,1$ t.

$$
\text { d) Schneelast. }
$$

Als wahrscheinlichste einseitige Schneelast Rour halb belastet sind und $1 / 4$ unbelastet ist, sei dies infolge der Einwirkung der Sonnenbestrahlung oder des Windes. Sieht man von der aus-
steifenden Wirkung der Ringdecken und von der Druckringverbindung der Binder ab, so würde in diesem Falle jeder Binder den gleichen Boren-

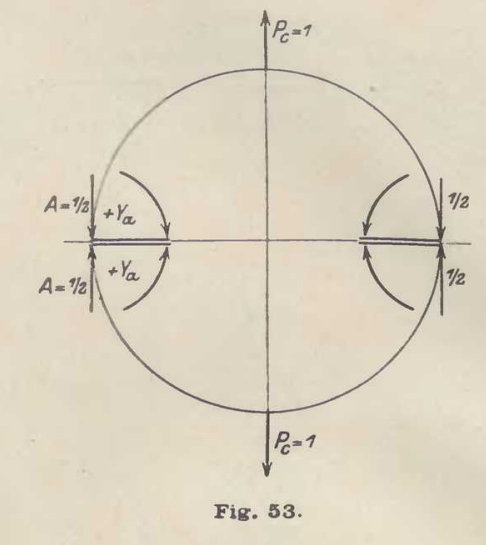

schub ausüben, und der Zugring würde keine Biegungsmomente erleiden. Der Bogenschub be trägt mit den Knotenlasten $P_{3}=2,6, P_{6}=2,0, \quad P_{9}$

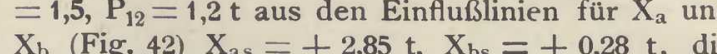
Auflagerdrücke $A_{s}=+5,86 t, B_{s}=1,44 t$, die Bie

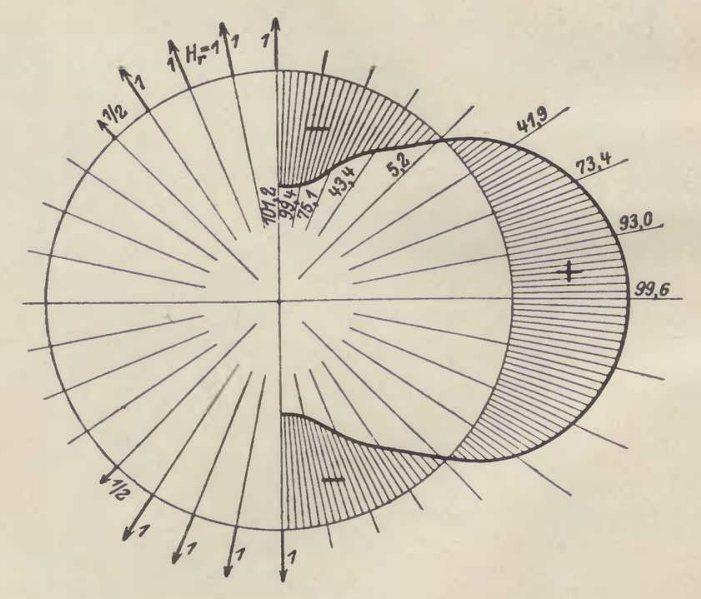

Fig. 54

gungsmomente des Binders sind in der Übersicl Für den Zugring tritt - wieder unter Vernachlässigung der Ringdecken - die ungünstigste Belastung dann ein, wenn nur etwa $1 / 4$ mit Schne

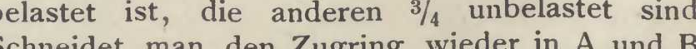
o erhält man für zwei gleich große entorecengesetzt gerichtete Krâfte $P_{c}=1$ in den Schnitten

\section{$-49$}

lediglich Verdrehungen, es wirkt also nur ein staisch unbestimmtes Moment $Y_{\mathrm{a}}$ (Fig. 53),

Man erhält in gleicher Weise wie frühe

$$
J_{a c}=-\frac{r^{2}}{2 E J}\left(\frac{\pi}{2}-1\right),
$$

Die Momente der Kuppelbinder aus EigenUewicht, Winddruck und Schneelast sind in der Größtwerte treten entweder bei Eigengewicht und
Winddruck oder bei Eigengewicht und Schneelast

$$
\begin{aligned}
v_{\mathrm{aa}} & =+\frac{\mathrm{r} \pi}{2 \mathrm{EJ}} \\
\mathrm{Y}_{\mathrm{a}} & =+\mathrm{r} \frac{7-2}{2 \pi}
\end{aligned}
$$

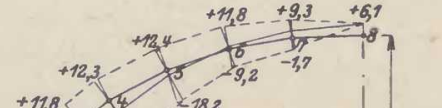
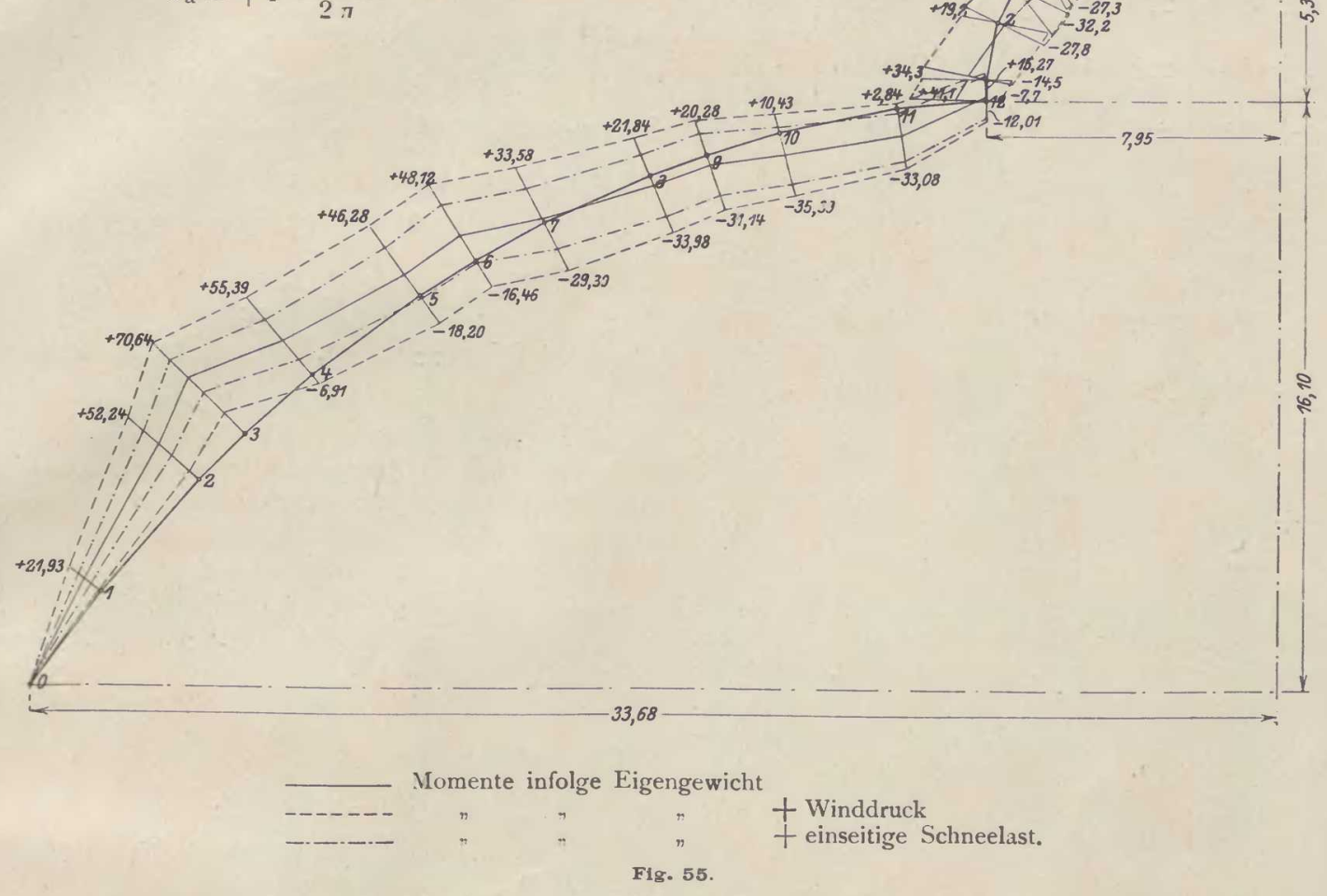

Das Moment in einem Punkte $r$ ist nun

$$
\begin{aligned}
\mathrm{M} & =\mathrm{M}_{0}+\mathrm{Y}_{\mathrm{a}} \mathrm{M}_{\mathrm{a}}, \\
& =-\frac{1}{2} \mathrm{r}(1-\sin \beta)+\mathrm{r} \frac{\pi-2}{2 \pi} \\
& =-\frac{\mathrm{r}}{2}\left(\frac{2}{\pi}-\sin \beta\right) .
\end{aligned}
$$

auf, ein gleichzeitiges Auftreten von einseitiger Schneelast und größ̂tem Winddruck muß als ausgeschlossen gelten.
Die Binder ha

Dit Bhy 1,05 haben am Scheitel einen QuerKämpfer von $1,20 \mathrm{~m}$ Höhe und $0,80 \mathrm{~m}$ Breite, am sind mit 13 Rundeisen von $30 \mathrm{~mm} \varnothing$ bewehrt, der Beton besteht aus 1 Z., 3 S., 3 Granitfeinschotler Man erhält mit dem Beiwerte $\frac{\mathrm{r}}{2}$ für die Punkte $\begin{aligned} & \text { Beton beanspruchungen im ungünstigsten Quer- } \\ & \text { Die Ber Ber } \\ & \text { schnitt } 3 \text { betragen unter Berucksichtigung der }\end{aligned}$ $\begin{array}{ccc}0 & 1 & 2 \\ -0,6366-0,4415 & -0,2539 & -0,0810\end{array}$

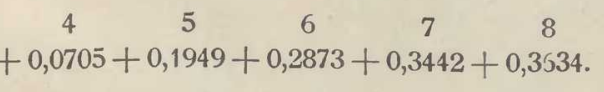
Für eine Viertelkreisbelastung sind die Binder und einanderlegen der einzelnen Belastungswirkun erhălt man die in Fig. 54 eingetragenen Werte schnitt 3 betrage
Doppelbewehrung

$$
\begin{aligned}
& \sigma_{\mathrm{b}}=55 \mathrm{~kg} / \mathrm{qcm} \\
& \sigma_{\mathrm{e}}=518
\end{aligned}
$$

Der Zugring erleidet aus Eigengewic $Z_{\mathrm{g}}=5,1 \cdot 94,26=\sim 480 \mathrm{t}, \mathrm{M}_{\mathrm{g}}=0$

aus Winddruck $\sim-39 \mathrm{t}, \mathrm{M}_{\mathrm{g}}=121,4 \mathrm{~m}$ $Z_{\mathrm{s}}=5,1 \cdot 2,85=\sim+15 \mathrm{t}, \mathrm{M}_{\mathrm{g}}=101 \mathrm{mt}$ 
50

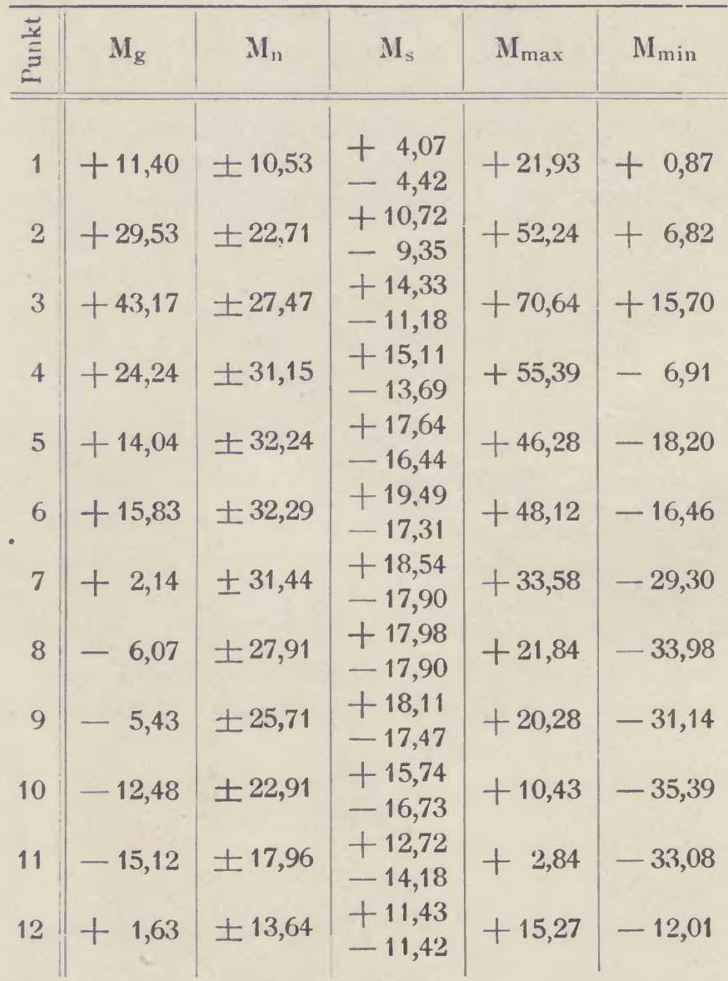

Der Zugring ist auf Vorschlag der Firma als 4 teiliger genieteter Trager ausgebildet worden,
dessen Gurte aus je 2 Fl. $365 / 13$ und 2 L $120 / 120 / 13$ dessen Vergitterung aus $L \mathbf{7 5 / 7 5 / 8}$ besteht (Fig. 56 ). Der Vollquerschnitt ist $617,2 q \mathrm{~cm}$, der Nutzquerschnitt $521,5 \mathrm{qcm}$. Die Beanspruchung beträg

$\sigma_{\mathrm{g}}=\frac{480}{521,5}=0,920 \mathrm{t} / \mathrm{qcm}$

$\sigma_{\mathrm{w}}=-\frac{39}{521,5} \pm_{62000}^{12140}=-0,075 \pm 0.196={ }_{-0,271}^{+0,121 \mathrm{t} / \mathrm{qcm},}$ $\sigma_{s}=\frac{15}{521,5} \pm \frac{101}{62000}=+0,229 \pm 0,163=\left\{\begin{array}{l}+0,192 \\ -0,134\end{array}\right.$

$\sigma_{\max }=\sigma_{g_{1}} \mathrm{~s}=1,112 \mathrm{t} / \mathrm{qcm}$

Die Stoßdeckung ist sehr reichlich bemesser.

3. Druckring und Laterne

Der Druckring erleidet aus Eigengewicht eine ormalkraft

$=-5,1\left(X_{a}+X_{b}\right)=-5,1(94,26+6,86)=-516 t$ Das Moment am Binderanschlusse beträgt $\mathrm{M}_{\mathrm{g}}^{\prime}=+1,63 \mathrm{mt}$

nimmt nach 5.38 der Latencibinder au $\mathrm{M}_{1}=(0,166+0,187 \cdot 3,60) \mathrm{M}_{\mathrm{g}}^{\prime}=0,839 \mathrm{M}_{\mathrm{g}}^{\prime}$ so daß̧ für den Druckrino verbleibt
$\mathrm{N}^{\prime \prime}{ }_{\mathrm{g}}=0,161 \mathrm{M}_{\mathrm{g}}=0,26 \mathrm{mt}$

in der Binderebene, oder

$\mathrm{M}_{\mathrm{g}}=5,1 \mathrm{M}_{\mathrm{g}}{ }^{\prime}=1,3 \mathrm{mt}$

der Kreisachse.

Für Winddruck war gefunden

$\mathrm{M}_{\mathrm{w}}^{\prime}= \pm 13,64 \mathrm{mt}$

davon nimmt auf der Druckring (s. S. 41

$\mathrm{M}^{\prime \prime}{ }_{\mathrm{w}}=0,546 \mathrm{M}^{\prime}{ }_{\mathrm{w}}= \pm 7,4 \mathrm{mt}$

der Binderebene oder

$\mathrm{M}_{\mathrm{w}}=5,1 \mathrm{M}^{\prime \prime}{ }_{\mathrm{w}}= \pm 38,0 \mathrm{mt}$

in der Kreisachse.

Für Schneelast war gefunden

$\mathrm{I}_{\mathrm{s}}^{\prime}=\sim \pm 11,42 \mathrm{mt}, \quad \mathrm{D}_{\mathrm{s}}^{\prime}=\mathrm{X}_{\mathrm{as}}+\mathrm{X}_{\mathrm{bs}}=3,13 \mathrm{t}$

$\mathrm{M}^{\prime \prime}{ }_{\mathrm{s}}=0,546 \mathrm{M}_{\mathrm{s}}= \pm 6 \mathrm{mt}$

$\mathrm{M}_{\mathrm{s}}=5,1 \mathrm{M}_{\mathrm{s}}^{\prime \prime}= \pm 32,0 \mathrm{mt}, \mathrm{D}_{\mathrm{s}}=5,1 \mathrm{D}_{\mathrm{s}}^{\prime}=\sim 16,0 \mathrm{t}$

Ferner ruft die einseitige Schneelast in der

Derer

as Moment für die Krafteinheit des Bogenchubes nach $2 \mathrm{~d}$

$$
\begin{array}{r}
M_{c}=\frac{r}{2}(0,6366+2 \cdot 0,4415+2 \cdot 0,2539 \\
+2 \cdot 0810-0,0705) \\
=+\frac{r}{2} 2,1189=8,42
\end{array}
$$

fir $\mathrm{D},=$

$\mathrm{M}_{\mathrm{c}}=+26,4 \mathrm{mt}$

Nit $\mathrm{F}^{\prime}=1,905 \mathrm{qm}, \mathrm{J}_{1}=0,3860 \mathrm{~m}^{4}, \mathrm{~W}_{1}=0,702 \mathrm{~m}^{3}$

$\sigma_{\mathrm{g}}=-\frac{516}{1,9015} \mp \frac{1,3}{0,283}=-271 \mp 46=-317 \mathrm{t} / \mathrm{qm}$,

$\sigma_{w}=\mp \frac{38}{0,283}=$

$\sigma_{s}=-\frac{16}{1,905} \mp \frac{32}{0,283} \mp \frac{26,4}{0.702}$

$$
=-8 \mp 114 \mp 37=-159
$$

$\sigma_{\mathrm{g}+\mathrm{w}}=-45,1 \mathrm{~kg} / \mathrm{qc1}$

$\sigma_{\mathrm{g}+\mathrm{s}}=-47,6$,

Zug tritt nirgends auf.

Nach S. 41 hat der Druckring bei einem ußseren Momente $\mathrm{M}=1$ aufzunehmen

$\mathrm{M}_{\mathrm{a}}=0,546 \mathrm{mt}$,

ent rechts und links vom Binderanschlusse beträgt also
\[ \mathrm{M}: 9=0,273, \]
51

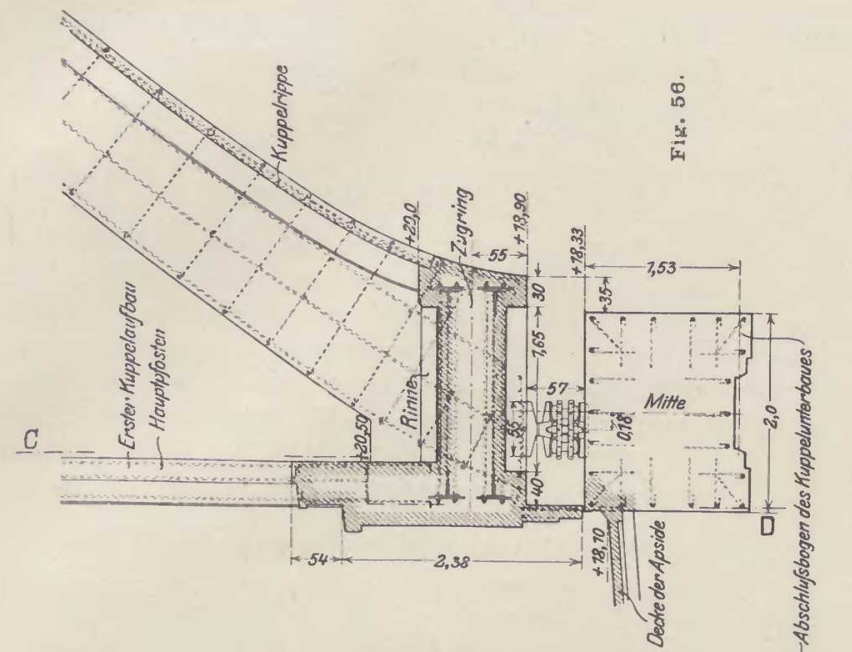

mit $\mathbf{M}_{\mathrm{w}}= \pm 53,3 \mathrm{mt}$ (s. unten) erhält man also $\quad M: s= \pm 14,6 \mathrm{mt}$ die größte Torsionsspannung beträgt $=\frac{9 \mathrm{~N} / 9}{2 \mathrm{~b}^{2} \mathrm{~h}}=36,2 \mathrm{t} / \mathrm{qm}$

hierbei sind die $4 \mathrm{am}$ stärksten von
Winde getroffenen Binder gen zusammengefarst gedacht worden. Der Laternenrahmen erhalt aus seirespannten Bogen in fur einen einermittelten Momente $\mathrm{M}_{\mathrm{g}}$, die Nitwirkung der Kuppelbinder konnte- Anges außer Betracht bleibe rahmen noch die Scher Laternen-
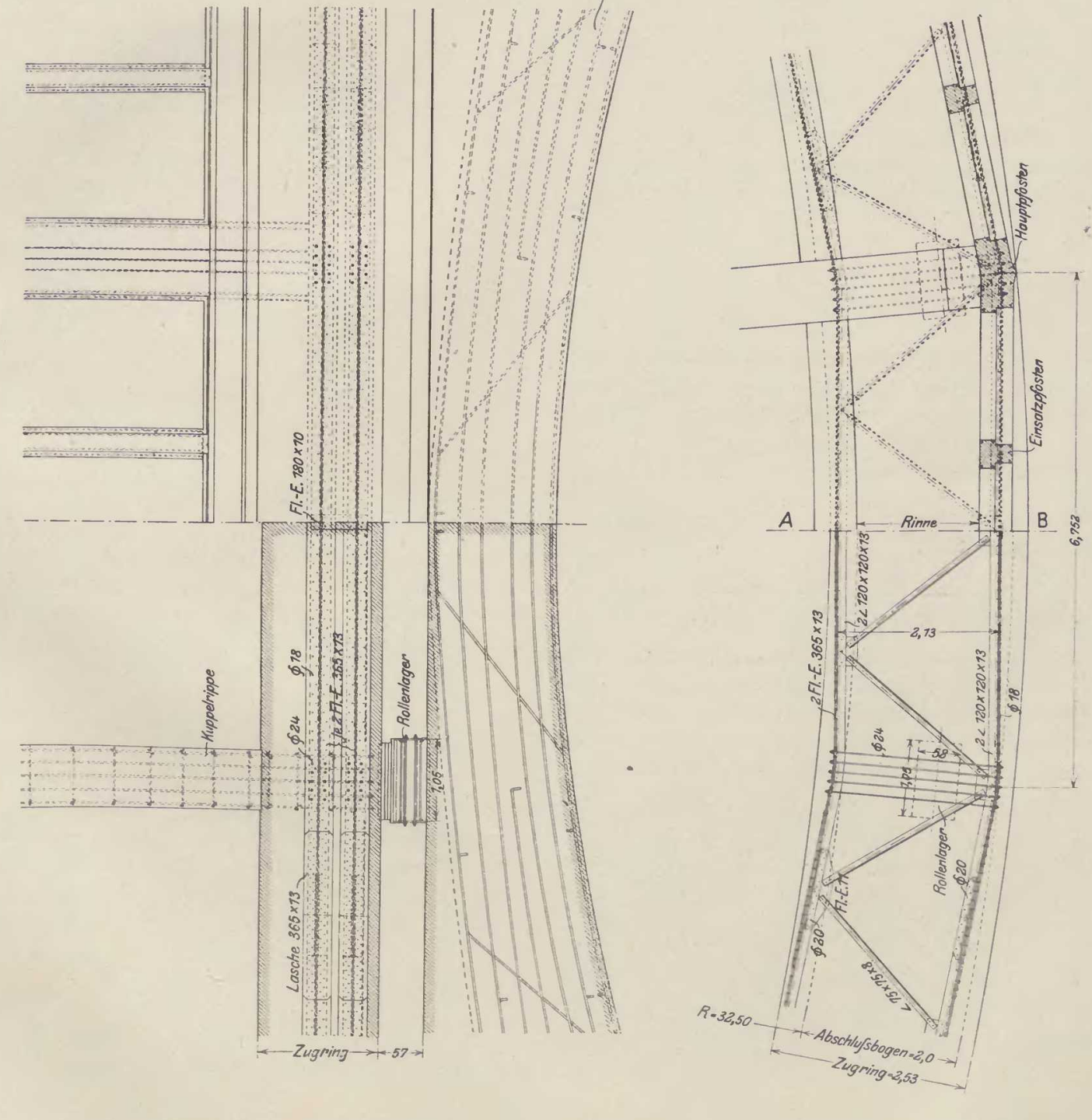
von ie 4 Bindern teilweise aufzunehmen, soweit diese nicht durch den Druck

$\mathrm{M}_{\mathrm{g}}=+4 \cdot 1,63=\sim 6,5 \mathrm{mt}$,

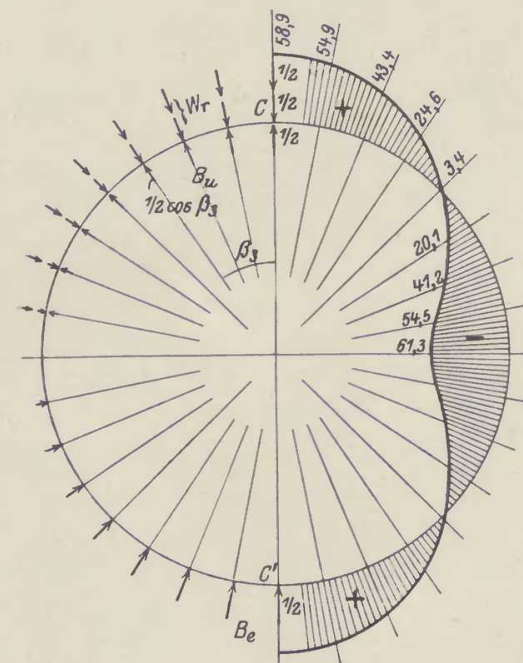

Die Laternenrahmen sind in selhr krättiger Schnitte 2 beträgt unter Berücksichtigung in

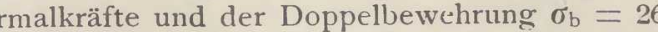
$\sigma_{\mathrm{e}}=928 \mathrm{~kg} / \mathrm{qcm}$

\section{Ringdecken.}

Sieht man zunächst von einer teilweisen Fensterbakten gebildeten Rahnmen ab, so haben die Ringdecken die Aufgabe, die Hälfte der Windkrafte von der Luv- nach de C wirken auf die Ringdecke in $\mathrm{C}$ und $\mathrm{C}^{\prime}$ einander entregengesetzt gerichtete gleich große wirken $\frac{1}{2} \cos \beta_{1}$ usw. Di Iomente fur die Lasteinheit in $\mathrm{C}$ und $\mathrm{C}^{\prime}$ sind au

$\mathrm{W}_{\mathrm{r}}=$ Gesamter Winddruck auf den Binder $\mathrm{r}$,
$\mathrm{B}_{\mathrm{u}}=$ Gegendrucke der Binder auf der Lurseite,
$\mathrm{B}_{\mathrm{B}}=$.

Fig. 57 .

welches ergibt

$x_{a}=+0,166 \cdot 6,5=+1,08 m$

und $\quad X_{c}=+0,187 \cdot 6,5=+1,215$ t,

$\mathrm{X}_{\mathrm{a}}$ und $\mathrm{X}_{\mathrm{c}}$ ergeben die in der folgenden Übersich ther binder das Scheitelmoment

$M_{w}= \pm 13,64(2 \cdot 0,995+2 \cdot 0,957)= \pm 53,3 \mathrm{mt}$ welches ergibt

$$
\mathrm{X}_{\mathrm{b}}= \pm 0,0572 \cdot 53,3= \pm 3,06 \mathrm{t},
$$

$\mathrm{x}_{\mathrm{b}}$ ergibt die fotrenten

Einseitige Schneelast kann außer Betracht

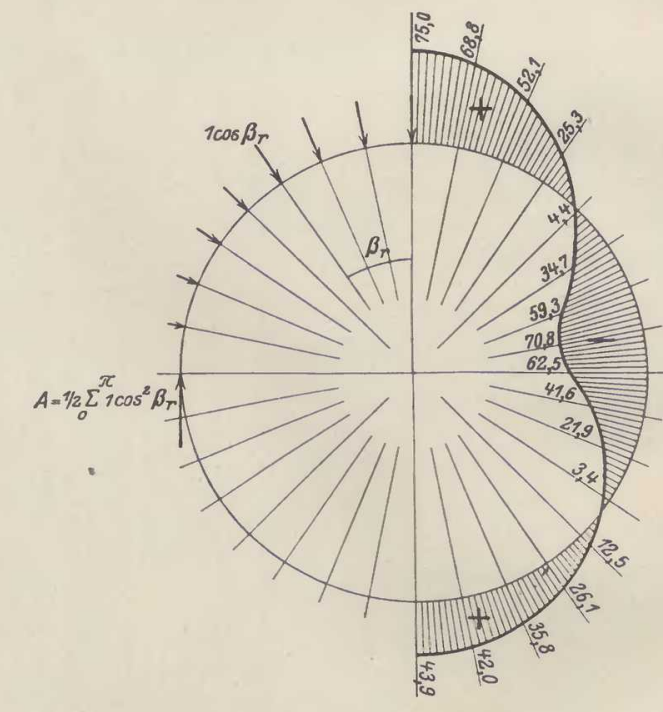
plizieren, durch Obereinanderlegen der einzelnen denen aus Wind bleiben.

\begin{tabular}{c||c|c|c|c|c|c}
\hline Punkt & $\mathrm{M}_{\mathrm{g}}$ & $\mathrm{M}_{\mathrm{g}}$ & $\mathrm{M}_{\mathrm{g}+\mathrm{g}^{\prime}}$ & $\mathrm{M}_{\mathrm{w}}$ & $\mathrm{M}_{\max }$ & $\mathrm{M}_{\min }$ \\
\hline \hline 0 & $+11,22$ & $+5,45$ & $+16,67$ & $\pm 24,4$ & $+41,1$ & $-7,5$ \\
1 & $+5,08$ & $+4,78$ & $+9,86$ & $\pm 24,4$ & $+34,3$ & $-14,5$ \\
2 & $-7,14$ & $+2,83$ & $-4,31$ & $\pm 23,5$ & $+19,2$ & $-27,8$ \\
3 & $-11,89$ & $+1,65$ & $-10,24$ & $\pm 22,0$ & $+11,8$ & $-32,2$ \\
4 & $-8,52$ & $+0,98$ & $-7,54$ & $\pm 19,8$ & $+12,3$ & $-27,3$ \\
5 & $-2,95$ & $+0,04$ & $-2,91$ & $\pm 15,3$ & $+12,4$ & $-18,2$ \\
6 & $+1,84$ & $-0,58$ & $+1,26$ & $\pm 10,5$ & $+11,8$ & $-9,2$ \\
7 & $+4,72$ & $-0,96$ & $+3,76$ & $\pm 5,5$ & $+9,3$ & $-1,7$ \\
8 & $+7,10$ & $-1,03$ & $+6,07$ & 0 & $+6,1$ & $+6,1$
\end{tabular}

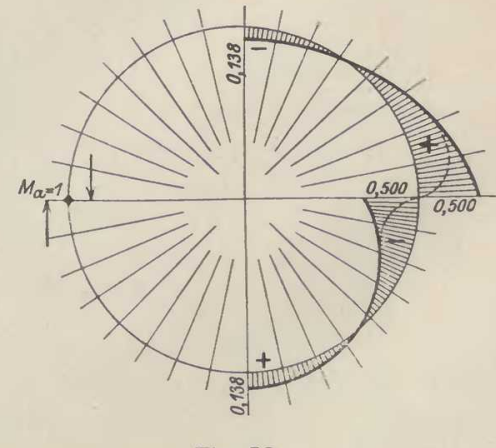

Fix. 5 .
Belastungssysteme erhält man die folgenden Zahlen Beiwert $\frac{\mathrm{r}}{4}$ ) und mit $\mathrm{W}=5,3 \mathrm{t}$ die in Fig. 57 eingetragenen Werte für die erste Ringdecke.

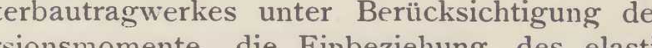
orsionsmomente, die Einbeziehung des elastcher

\begin{tabular}{|c|c|c|c|c|c|c|c|c|c|c|}
\hline \multirow{2}{*}{ Last } & \multirow{2}{*}{ im Punkte } & \multicolumn{9}{|c|}{ M o ment im Punkte } \\
\hline & & 0 & 1 & 2 & 3 & 4 & 5 & 6 & 7 & 8 \\
\hline $\begin{array}{l}1,00 \\
0,981\end{array}$ & $\begin{array}{l}0 \\
1\end{array}$ & $\begin{array}{l}+0,6366 \\
+0,433\end{array}$ & $\begin{array}{l}+0,4415 \\
+0,625\end{array}$ & $\begin{array}{l}+0,2539 \\
+0,433\end{array}$ & $\begin{array}{l}+0,0810 \\
+0,249\end{array}$ & $\begin{array}{l}-0,0705 \\
+0,080\end{array}$ & $\begin{array}{l}-0,1949 \\
-0,069\end{array}$ & $\begin{array}{l}-0,2873 \\
-0,191\end{array}$ & & \\
\hline 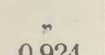 & $1^{*}$ & $+0,433$ & $+0,249$ & $+0,080$ & $-0,069$ & $-0,191$ & $-0,283$ & $-0,338$ & $-0,356$ & $-0,338$ \\
\hline 0,924 & $2^{2 *}$ & usw. & usw. & usw. & usw. & usw. & usw. & usw. & usw. & usw. \\
\hline & & & & & & & $-0,489$ & $-1,004$ & & \\
\hline
\end{tabular}

Der zweite Fall in der Übertragung der Ringdecken ist der, daß nur ein Teil des Windes durch die Binder in den Zugring geleitet wird, der andere Tie Rahmenwirkung der Binter der Posten und rippen (siehe axonometrische Projektion Fig. 33). Die Momente für den Grenzfall, daß die Rahmen den gesamten Winddruck aufnehmen, werden

Mit $W=5,30 \mathrm{t}$ erhält man für die erste Ring
cke die in Fig. $57 \mathrm{~b}$ eingetragen

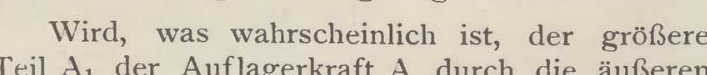
Teil $A_{1}$ der Auflagerkraft $\mathrm{A}$ durch die äußeren
Pfosten, der kleinere $A_{2}=A$ Rahmenwirkung der Binder samt Versteifunos. rippen nach unten geleitet, so tritt bei $\mathrm{A}$ noch in Moment $\left(A_{1}-A_{2}\right)$ a auf (Fig. 51 rechts). Ein Ioment 1 in A ergibt die in Fig. 58 eingetragene Momentenlinie, wobei die gestrichelte Linie gilt,
wenn eine grölsere Anzahl Pfosten an der Kraftaufnahme teilnimmt. Diese Momente wirken in allen in Frage kommenden Punkten entlastend. betragen in der ersten Ringdecke Grenzwerte, $-63,1 \mathrm{mt}$ bei Fall a und $+75,0$ und $-70,8 \mathrm{mt}$ bei Fall b. Die Ringdecken werden gebildet aus der $8 \mathrm{~cm}$ starken Deckenplatte als Steg, der

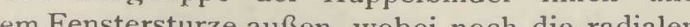
Deckenunterzüge aussteifend wirken

Die Pfosten, Sohlbänke und Fenstersturze sind außer für Eigengewicht noch für die Übertragung der Windkräfte als Rahmenträger berechnet worden, wobei funf Rahmen als an der Kraftubertragung

Die vorstehend wiedergegebenen statischen
Berechnungen, also vor allem die Berechnung des

e Berechnung der Ringdecken sim estellt worden. Von der Ana sind, wie bereits erwähnt, die konstruktive asbinting, insbesondere die Festlegung der Berechnung des Windrahmens und alle cht aufgeführten Bauteile erschiedene zu den Untersuchungen der Bau-

TEIL II.

Von Prof. Dr.-Ing. W. Gehler (Dresden).

Die konstrultive Gestaltung und die Ausführung.

Neben dem architektonischen Entwurt und er statischen Berechnung war vor allem auch

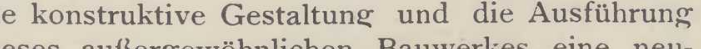
tige und äußerst verantwortungsvolle Aufgabe eren Losung einen beträchtlichen Schritt vorwärts der Entwicklung des Eisenbetonbaues bedeutet. die ausfuthrende Firma und deren Leiter, strafrechto daher zunähst ein edürfnis, sich ein klares Bild zu verschaffe ther die Eigenart der Kraftwirkungen, über die Möglichkeit der Beherrschung dieser ungewöhnlic (a) nderen Baustoff, vollständig an Ort und Stelle

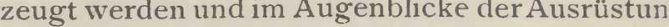


A. Vorerörterunter Kuppel des Brunellesco vom Dom in Um die Eigenart der Aufgabe richtig zu be- 1590, endlich die St. Pauls Kathedrale in London etwas ls außercewöhnlich erscheinen ließen. Die Schwie- Werke des Orients an, als deren bekannteste die rigkeiten für die konstruktive Gestaltung und die kühne und elegante Kuppel der Sophienkirche in Ausführung lagen einmal in den außerordent- Konstantinopel mit 31,5 m Lichtweite zu nenne Zeit der christliche

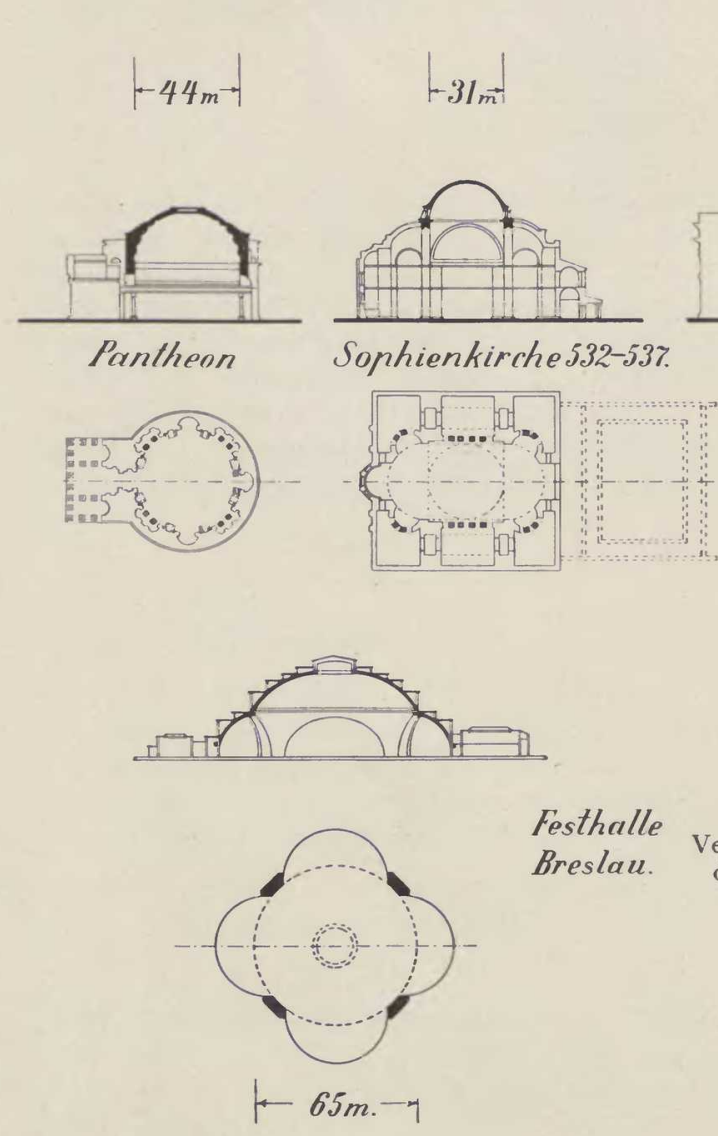

aber in der statischen Eigenart des Kuppelunterbaues.

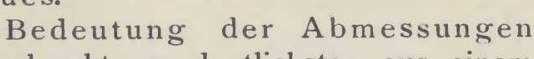
Óberblick über die bisher ausgeführten massiven Kuppeln hervor, die als Denksteine in der Baugeschichte bekannt sind. In Ubersicht $I$ sind diegestellt. Allem voran steht mit $44 \mathrm{~m}$ Iichtweite das Pantheon, dieses bewundernswerte Meisterwerk altrớmischer Baukunst, wahrscheinlich aus der Blütezeit romischer Wobtechnik unter Kaise

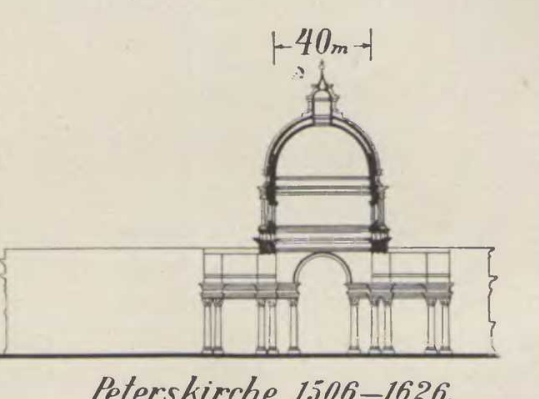

Peterskirche 1506-1620.

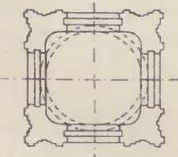

Massstab.

Vergleich der Breslauer Festhalle mit
den alten weitgespannten Kuppeln.

waren in den letzten 537 unter Kaiser Jus-
tinian I., von den Baumeistern Anthemios von Tralles und
Isidor von Milet. Eine einen halbe weite, nämlich $32 \mathrm{~m}$ weist die noch vor kämpfte SelimieMoschee in Adrianpel auf, erbaut von auch die Moschee des $26 \mathrm{~m}$ Lichtweite $\mathrm{z}$ zuschreiben ist. Dies
und die fol genden kleineren Kuppeln stammen der Blütezeit türkischer Bauzum 18.Jahrhundert* In dem neuen Bau
stofti,dem Eisenbeton, bis zu einer Spannweite von $33,1 \mathrm{~m}$ bei der (t. Blasien**), immerhin bliebe aber die drei großen italienischen Kuppeln bisher

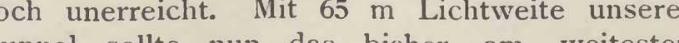
(a) Bauwerk, das Pantheon, um etesten älfte dieses Maßes übertroften werden (verge

Diese Angaben uber die orientalischen Bauten sind
vorzüglichen grundlegenden Werke von Corneli (in) entnommen. **) Ausgeführt von Dyckerhoff \& Widmann, A.-G.,
Karlsruhe; siehe Deutsche Bauzeitung 1912, Mitteilungen
Übersicht I. Weitgespannte massive Kuppeln.

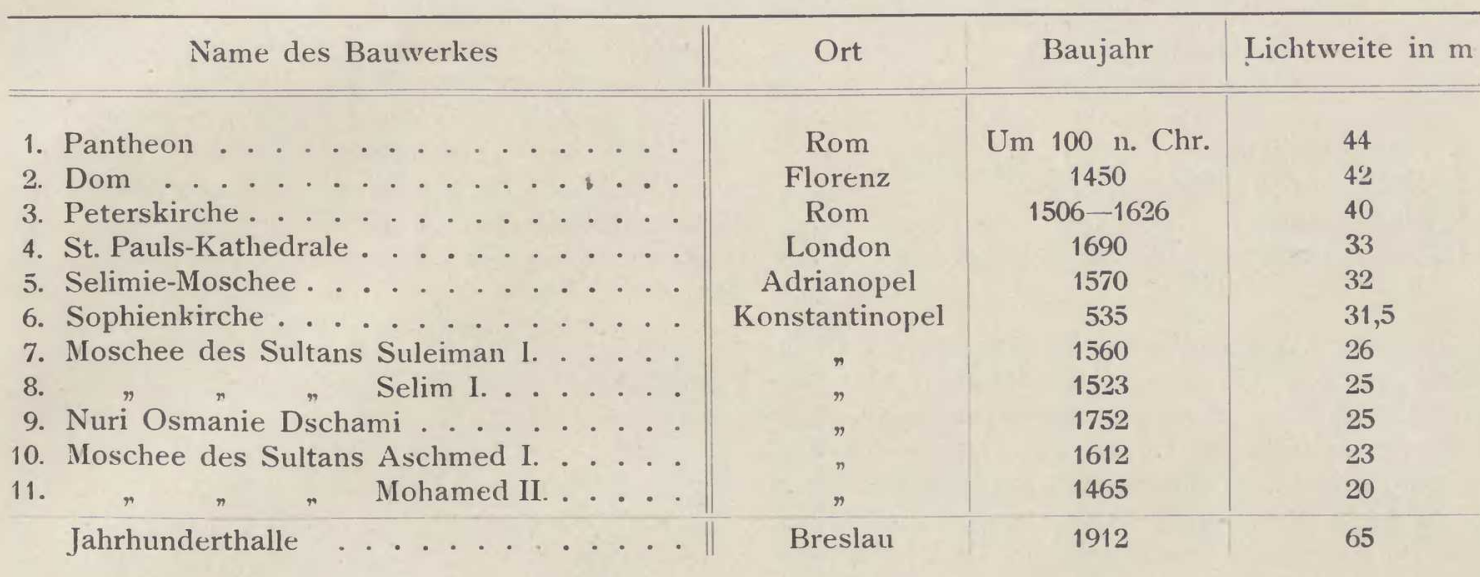

Selbst für Kuppeln in Eisen ist dieses Maßs Weise das Eigengewicht einer massiven Kuppe on $65 \mathrm{~m}$ mur bei wenigen Bauwerken erreicht

Ausstellungskuppel in Lyon*), dem Zeltdach der bekannten Rotunde in Wien**) und einer Anzahl neuerer Gasbehalterumbauten

sen den Vorteil bot, probt waren und die Montage leins wiederholt erSchwierigkeiten erwarten ließ\}, wurden bei der Ausschreibung im Jahre 1911 als Baustoft sowohl Bedingung daß sämtliche eisernen Tragteile mit Rücksicht auf die Feuersicherheit ummantelt werden müßten, wurde jedoch die Ausfuhrung in Eisen nicht wesentlich wirtschaftlicher beidung den Eisenauf and beträchtlich erhöhte und der Eisenbeton für die Aufnahme der Druckkräfte in der Kuppel, den Hauptbögen und den Strebebogen sehr geeignet, hier also ist. Nur haftet der Ausführung in Fiser Baustoff bedeutsame Umstand an, daß mit wachsender Stützweite das Eigengewicht stark zunimmt. $\mathrm{Zu}$ dem war der Einfluß des Eigengewichtes in weil dadurch die Rüstung für Kuppel ueduterbau wesentlich erschwert wurde und die Ausrüstung bei einem bisher in solchen Abmessungen noch nicht ausgeführten Bauwerk ein gewisses Wagnis den Ansporn gibt. Es ist daher auch für künftioge Ausführungen die Erörterung der Frage wertvoll, in 'welcher

*) Siehe M. Foerster, Eisenhochbauten, III. Auflage. ***) Siehe u. a. W. Gellerer, Gasbehälter Reick, Armierter durde ter verfasser das Eigengewicht für ine Reihe von Kuppeln ermittelt. Diese
trachtung führte zu dem Ergebnis, daß das

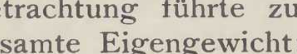

a) für Vollkuppeln $\mathrm{G}=\mathrm{Cl}^{2} \mathrm{f} \ldots \ldots$ b) Rippenkuppeln $\mathrm{G}=\mathrm{C}_{1} 1^{2}+\mathrm{C}_{2} 1^{2} \mathrm{f}$. (2 genommen werden $\left.\operatorname{kann}^{*}\right)$. Dabei bedeutet lichte Pfeilhöhe ebenfalls in Metern und $f$ die Ausdruck $\left(\mathbb{l}^{2} \mathrm{f}\right)$ ist daher nach Fig. 60 der Raum-

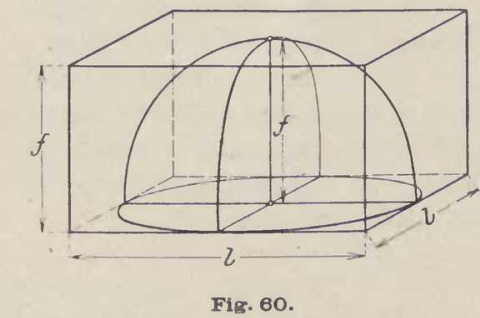

inhalt des prismatischen Körpers zu verstehen der dem von der Kuppel umschlossenen lichten Raume umschrieben ist. Die Beiwerte $\mathrm{C}$ und $\mathrm{C}_{2}$ Gewichte eines jeweils die im folgenden erorterten Körpers in $\mathrm{kg}$ ausgedrückt.

Für die Rippenkuppel, Gl. (2), empfiehlt e *) Unter Vollkuppeln sind hierbei Kuppeln verstanden,
deren Tragwerk die vollstiandig geschlossene Oberflähe Rotationskoorpers bildet, im Gegensatz $\mathrm{zu}$ de 
$-56$

Übersicht II. Weitgespannte eiserne Kuppeln

\begin{tabular}{|c|c|c|c|}
\hline Name des Bauwerkes & Ort & Baujahr & Lichtweite in $m$ \\
\hline $\begin{array}{l}\text { 1. Ausstellungskuppel ...... } \\
\text { 2. Rotunde des Industriepalastes } \\
\text { 3. Gasbehälter } \ldots \ldots \\
\text { 4. Sängerhalle }\end{array}$ & $\begin{array}{l}\text { Lyon } \\
\text { Wien } \\
\text { Dresden-Reick } \\
\text { Frankfurt a. M. }\end{array}$ & $\begin{array}{l}1894 \\
1873 \\
1908 \\
1909\end{array}$ & $\begin{array}{r}110 \\
102 \\
65 \\
63\end{array}$ \\
\hline
\end{tabular}

decke nebst Aufbauten von dem soeben betrach-
teten zweiten Gliede der Gleichung, dem Gewichte des Kuppeltragwerkes abzutrennen. Das Eigengewicht zu bildet, sowie das Gewicht etwaiger Aufbauten und der Laterne hängen nicht von dem Luftraume $\left(1^{2} \cdot f\right)$ (s. Fig. 60) ab, sondern von der zu überdeckenden Ficher Körpers der Fig. 60, also das dem Kuppelrnng um-
schriebene Quadrat einen Anhalt bietet. Der Beiwert $\mathrm{C}_{1}$ entspricht dann in $\mathrm{kg} / \mathrm{qm}$ ausgedrückt
dem Gewicht der über diese Fläche gleichmäßig verteilt gedachten Lasten der Dachdecke nebst führungsarten, von der leichtesten Glaseindeckung zwischen eisernen Sprossen bis zu den schweren Aufbauten unserer Festhalle, den jeweiligen baltissen angemessen eingesezt werden Bemerkenswert ist hierbei, daß nach beide werk z. B. bei $\mathrm{f}=\frac{1}{2}$ in die Form $\mathrm{G}=\mathrm{C}^{\prime} \cdot \mathrm{l}^{3}$ gebracht werden kann, also mit der dritten Potenz der Lichtweite nach einer kubischen Parabe wächst. Dementsprechend vergroßern sich auch etwa, wie häufig bei ebenen Tragwerken, nur mit dem Quadrat der Stützweite, sondern in wesent-

*) Wollte man bei dem Gewichte der Dachdecke
ebenfalls das Pfeilverhälthis der Kuppel berücksichtigen, $G=C_{1} 1 \mathrm{f}+\mathrm{C}_{2}$

abzuändern. Da aber fü die Ausfürung in Eisenbeton
Halbbreiskuppeln wohl nur selten in Frage kommen und geringer Grenzen schwaknken dürfte, andererseits aber
âhnlich wie bei sonstigen Dachformen das Eigengewicht überdeckte Grundfläche bezogen wird, empfiehtt es sich wohl, hier von der Beruckssichtigung des Peilvertaltinisses des Daches bezogen auf die Grundfläche von dem statiscl räumlich wirksamen Tragwerk unterschieden wird,
leiechter
In Fig. 61 ist für die in der Übersicht III zIIKuppeln jeweils die Licht-

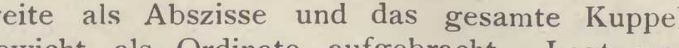
urch den Punkt II (Peterskirchenkuppel) Legt ma

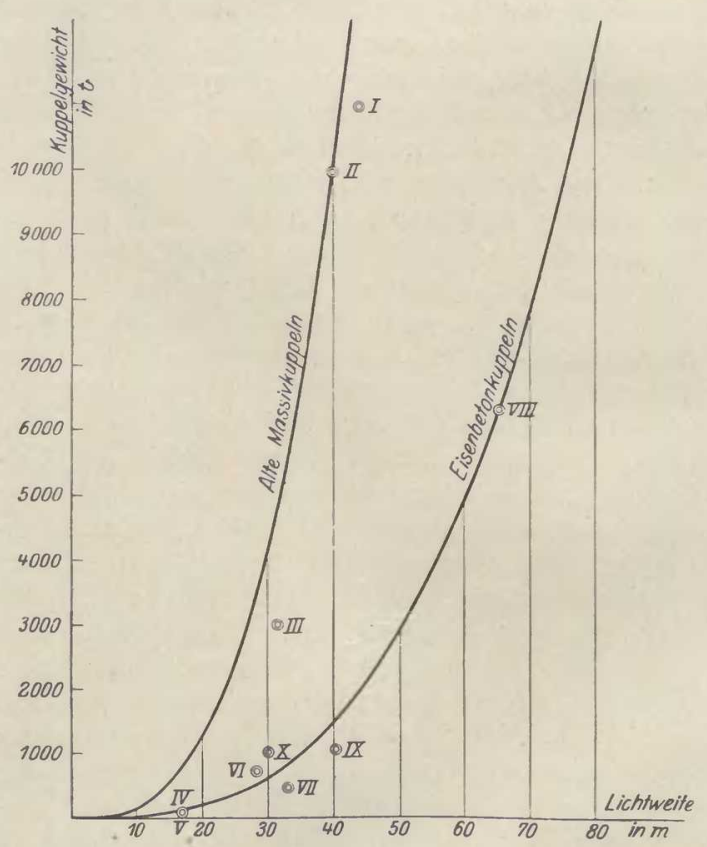
Fig. 61

Abhängigkeit des Kuppelgewichts von der Lichtweite.

kurve der Eisenbetonkuppeln von der der alten massiven Kuppeln wesentlich abweicht. Haupt-

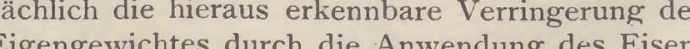

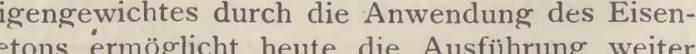
gespannter Kuppeln als in früherer Zeit. Aus ieser Darstellung sieht man aber auch deutlich, die übriwicht weit hinter sich laßst.
In Ubersicht III sind endlich für eine Reihe alter und neuer Kuppeln die Gewichte zusammenpeln, sowie $C_{1}$ und $C_{2}$ für Rippenkuppeln ermittelt Kuppeln I und II der Festwert C etwa 450 urd $300 \mathrm{~kg} / \mathrm{cbm}$ und bei der Sophienkirche in Konstantinopel (vergl. auch Fig. 59) beretts nur noch $200 \mathrm{~kg} / \mathrm{cbm}$ beträgt, schwankt dieser Wert be den neueren Eisenbetonkuppeln nur zwischen Festwert $C_{2}$ für das Trag werk der Rippenkuppeln zwischen 50 und $60 \mathrm{~kg} / \mathrm{cbm}$. Nur bei der Kuppe kuppel, also keinc Dachlast zu tragen hat, ergab sic $\mathrm{C}_{2}=40 \mathrm{~kg} / \mathrm{cbm}$. Diese auffallende Gesetzmäßri keit der Festwerte $C_{\text {und }} C_{2}$ ermogglicht uns tro hältnisse, das Gewicht von Kunpeln mit Hilfe der oben vorgeschlagenen Formeln rasch zu schatzen. Bei Rippenkuppeln ist dabei jedoch für das Gewicht der Dachdecke und der Aufbauten der Feswert $C_{1}$ in $\mathrm{kg} / \mathrm{m}$ der leichtesten Eindeckung mit Glas zwische Eisensprossen (vergl. Nr. VI) mit $C_{1}=50 \mathrm{~kg} / \mathrm{qm}$ bei mitte mit $250 \mathrm{~kg} / \mathrm{ms}$. dachdecke mit $250 \mathrm{~kg} / \mathrm{qm}$ und endich bei schwere $600 \mathrm{~kg} / \mathrm{qm}$. Durch Anwendung von Holz anstat von Eisenbeton für die Aufbauten ließse sich dieser Wert auf etwa $180 \mathrm{~kg} / \mathrm{qm}$ vermindern, ohn
daß dabei die Feuersicherheit des eigentliche
Eisenbetontragwerkes beeinträchtiogt würde.

Um darzutun, wie groß der Einflufs des Gewichtes der Dachdecke, also des Festwertes ist, wurde am Schluis der Obersicht III für eine von $1 / 4$ das Gesamtgewicht der Kuppel nachein-
ander unter Annahme von $C_{1}=50,250$ un $560 \mathrm{~kg} / \mathrm{qm}$ zu rund 13000,16000 und 21000 schătzungsweise berechnet. Diese Betrachtung weit gespanter Kuppeln lünftigh din a die Verminderung des Gewichtes für die Dachdecke und Aufbauten besonders bedacht zu sein. 2. Die statische Eigenart des Unterbaues geht am deutlichsten aus einem Vergleich

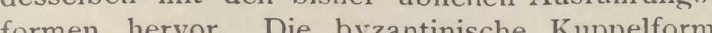
(s. Fig. 62) entsteht bekanntlich dadurch, daß sich
uber den vier Seiten eines Quadrates die vier *) Während die Maße für die drei alten Kuppeln I simmt werden konnten, sind die Angaben für Nr. IV bis Teil I, entnommen, wogegen die Grundmaße der übrigen neuesten Kuppeln unmittelbar von den ausfürenden Fir-
men stammen
$-$

Hauptbogen des Unterbaues erheben. Der Fuß ing der Kuppel ist dann ein Kreis, der diesen
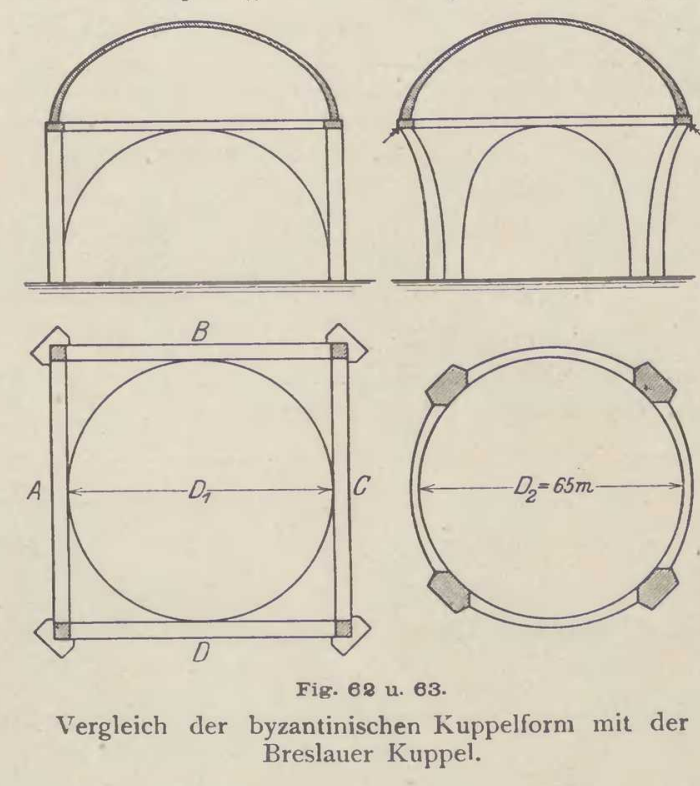

rult die Kuppel nur in vier Punkten A, B, C, D unmittelbar auf dem Bogen, während an den vier Ecken des Quadrates stark ausladende Zwickel
entstehen, die konstruktiv schwieriy auszubilden entstehen, die konstruktiv schwierig auszuridurch
sind. Bei den alten Bauten gelang es oft nur durch Aufbringung einer architektonisch ungünstig wirkenden, beträchtlichen Auflast diesen Zwickeln
den Verlauf der Stützlinien innerhalb des Querschnittes herbeizuführen. Bei unserem Kuppelden Ecken des Quadrates 13, Im Gegensatz hierzu ist der Unterbau der Brestauer Kuppel ein senkrecht stehender Zylinder to kann (Fig. 63). Die hierdurch entstehenden vie

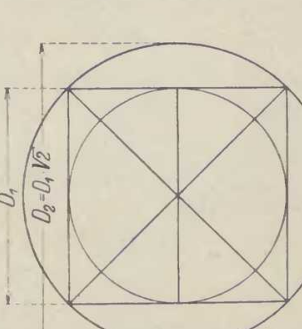

F19. 64 sondern Raumkurven vierter Ordnung. Durcl ese Gestaltung wir der Fußring der Kuppel auf seinem ganzen
Umfang unmittelbar auf diesem stehenden $Z_{y-}$ vier Hauptbogen daaußsen zu fallen und müssen da- 
$-58-$

Gelt man nach Fig. 64 in beiden Fällen von dem gleichen Quadrat aus, dessen Eckpunkte die Fläche die von der Kuppel selbst überdeckte

Ubersicht III. Festwerte des Kuppelgewichtes.

\begin{tabular}{|c|c|c|c|c|c|c|c|c|c|c|}
\hline \multirow{2}{*}{\multicolumn{6}{|c|}{ 1. Vollkuppeln. }} & $\begin{array}{c}\text { Licht- } \\
\text { weite } \\
1\end{array}$ & $\begin{array}{c}\text { Pfeil- } \\
\text { höle } \\
\text { f }\end{array}$ & & $\begin{array}{l}\text { Kuppel- } \\
\text { gewicht } \\
\text { G }\end{array}$ & $\begin{array}{l}\text { Fest- } \\
\text { wert } \\
\text { C }\end{array}$ \\
\hline & & & & & & in $\mathrm{m}$ & in $\mathrm{m}$ & & in $\mathrm{t}$ & $\mathrm{kg} / \mathrm{cbm}$ \\
\hline \multicolumn{6}{|c|}{$\begin{array}{l}\text { A. Alte massive Mauerwerkskuppeln } \\
\text { I. Pantheon in Rom . . . P } \\
\text { II. Peterskirche in Rom. } \\
\text { III. Sophienkirche in Konstantinopel }\end{array}$} & $\begin{array}{l}44 \\
40 \\
31, \overline{5}\end{array}$ & $\begin{array}{l}12,3 \\
20,0 \\
15,8\end{array}$ & & $\begin{array}{l}\text { wa } 11000 \\
\Rightarrow \quad 10000 \\
\Rightarrow \quad 3000\end{array}$ & $\begin{array}{l}450 \\
300 \\
200\end{array}$ \\
\hline \multicolumn{5}{|c|}{$\begin{array}{r}\text { B. Neuere Vollkuppeln aus Eisenbeton } \\
\text { IV. Armeemuseum in München (äufere Kuppel) } \\
n \\
\text { V. Universität München (elliptischere Grundrifi) }\end{array}$} & \multicolumn{2}{|c|}{$\left.\begin{array}{c}16,8 \\
16,1 \\
\{6,8\} \\
13,0\end{array}\right\}$} & $\begin{array}{l}8,4 \\
8,05\end{array}$ & & $\begin{array}{l}90 \\
87 \\
94\end{array}$ & $\begin{array}{l}38 \\
42 \\
48\end{array}$ \\
\hline \multirow{3}{*}{\multicolumn{2}{|c|}{ 2. Rippenkuppeln. }} & \multirow{3}{*}{$\begin{array}{l}\text { Art der } \\
\text { Decke und } \\
\text { Auflast }\end{array}$} & \multirow[b]{2}{*}{$\begin{array}{l}\text { Licht- } \\
\text { weite } \\
=1\end{array}$} & \multirow[b]{2}{*}{$\begin{array}{c}\text { Pfeil- } \\
\text { höhe } \\
f\end{array}$} & \multicolumn{2}{|c|}{ Gewicht } & \multicolumn{2}{|c|}{ Festwerte } & \multirow{2}{*}{\multicolumn{2}{|c|}{ Ausgeführt von }} \\
\hline & & & & & \begin{tabular}{|l} 
der \\
Dach- \\
decke \\
$\mathrm{G}_{1}$
\end{tabular} & $\mid \begin{array}{c}\text { des } \\
\text { Trag- } \\
\text { wer- } \\
\text { ees } \\
\text { k } \\
\mathrm{G}_{2}\end{array}$ & $C_{1}$ & $\mathrm{C}_{2}$ & & \\
\hline & & & in $m$ & in $\mathrm{m}$ & in $\mathrm{t}$ & in $\mathrm{t} / \mathrm{k}$ & $\mathrm{kg} / \mathrm{qm} / \mathrm{k}$ & kg/cbm | & & \\
\hline \multirow{2}{*}{\multicolumn{2}{|c|}{$\begin{array}{l}\text { VI. Passage Friedrichstraße } \\
\text { Berlin ........ } \\
\text { VII. Kirche St. Blasien (nur | } \\
\text { Innenkuppel). . . }\end{array}$}} & $\begin{array}{c}\text { Glas mit } \\
\text { Eisensprossen }\end{array}$ & 28,4 & 14,20 & 40 & 690 & 50 & \multirow{5}{*}{\multicolumn{3}{|c|}{$\begin{array}{c}\text { Czarnikow \& Co., } \\
\text { Berlin 1908 } \\
\text { Dyckerhoff \& } \\
\text { Widmann A.-G., } \\
\text { Karlsruhe 1911 } \\
\text { Dyckerhoff \& } \\
\text { Widmann A.-G., } \\
\text { Dresden 1912 } \\
\text { Dücker \& Co., } \\
\text { Düsseldorf 1912 } \\
\text { Kell \& Löser, } \\
\text { Leipzig 1913 }\end{array}$}} \\
\hline & & $\left|\begin{array}{c}\text { Eisenbeton- } \\
\text { decke (kein } \\
\text { Dach) }\end{array}\right|$ & \}$^{33,1}$ & 5,25 & 210 & 230 & 200 & & & \\
\hline VIII. & Festhalle Breslau .... & $\left\{\begin{array}{c}\text { Eisenbeton- } \\
\text { dach mit Auf- } \\
\text { bauten }\end{array}\right\}$ & \}$^{65,0}$ & 16,10 & 2.370 & 3970 & 560 & & & \\
\hline & Pumpwerk Alte Emscher & $\left\{\begin{array}{c}\text { Eisenbeton- } \\
\text { dach }\end{array}\right\}$ & 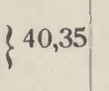 & 9,0 & 330 & 730 & 200 & & & \\
\hline & $\begin{array}{l}\text { Betonhalle der Int. Bau- } \\
\text { fachausstellung, Leipzig }\end{array}$ & $\begin{array}{l}\text { Eisenbeton- } \\
\text { dach }\end{array}$ & $\{30,0$ & 12,70 & 450 & 570 & 500 & & & \\
\hline \multirow{2}{*}{\multicolumn{3}{|c|}{$\begin{array}{l}\text { Kuppel von } 100 \mathrm{~m} \text { Lichtweite: } \\
\text { a) bei leichtem Glasdach. . . . . . . } \\
\text { b), bei Eisenbetondach ohne Auf bauten. . }\end{array}$}} & 100,0 & 25,0 & $\frac{500}{130}$ & $\frac{12500}{000}$ & 50 & 50 & & \\
\hline & & & 100,0 & 25,0 & $\frac{2500}{162}$ & $\frac{13750}{250}$ & 250 & 55 & & \\
\hline \multicolumn{3}{|c|}{ c) bei Eisenbetondach mit Aufbauten . } & 100,0 & 25,0 & $\frac{5600}{206}$ & $\frac{15000}{600}$ & 500 & 60 & & \\
\hline
\end{tabular}

Die hauptsächlichste konstruktive Schwierig- hätte zwar gegenüber der ausgeführten Anordnung jert $2 \cdot 3 \cdot 200 t=1200 t$ übertragen werden die be- ring der Kuppel die freie Drehbarkeit dieser zogen auf eine wagerechte durch die beiden Glieder eingeschränkt und dadurch bei einseitiger Kämpfermittelpunkte gelegte Linie an den Hebel- Schnee- und Windlast versteifend gewirkt hätten. armen von $3,85 \mathrm{~m}, 6,41 \mathrm{~m}$ und $7,65 \mathrm{~m}$ wirken. Andersets ware aber dadurch das Gesamtrag $7200 \mathrm{tm}$ für einen Bogen ergeben. Die Stand- Durch die scharfe Trennung von Kuppel und sicherheit des Bauwerkes beruhte daher haupt- Unterbau und die Anordnung von 32 Rollenlagern, săchlich aur der Zuw

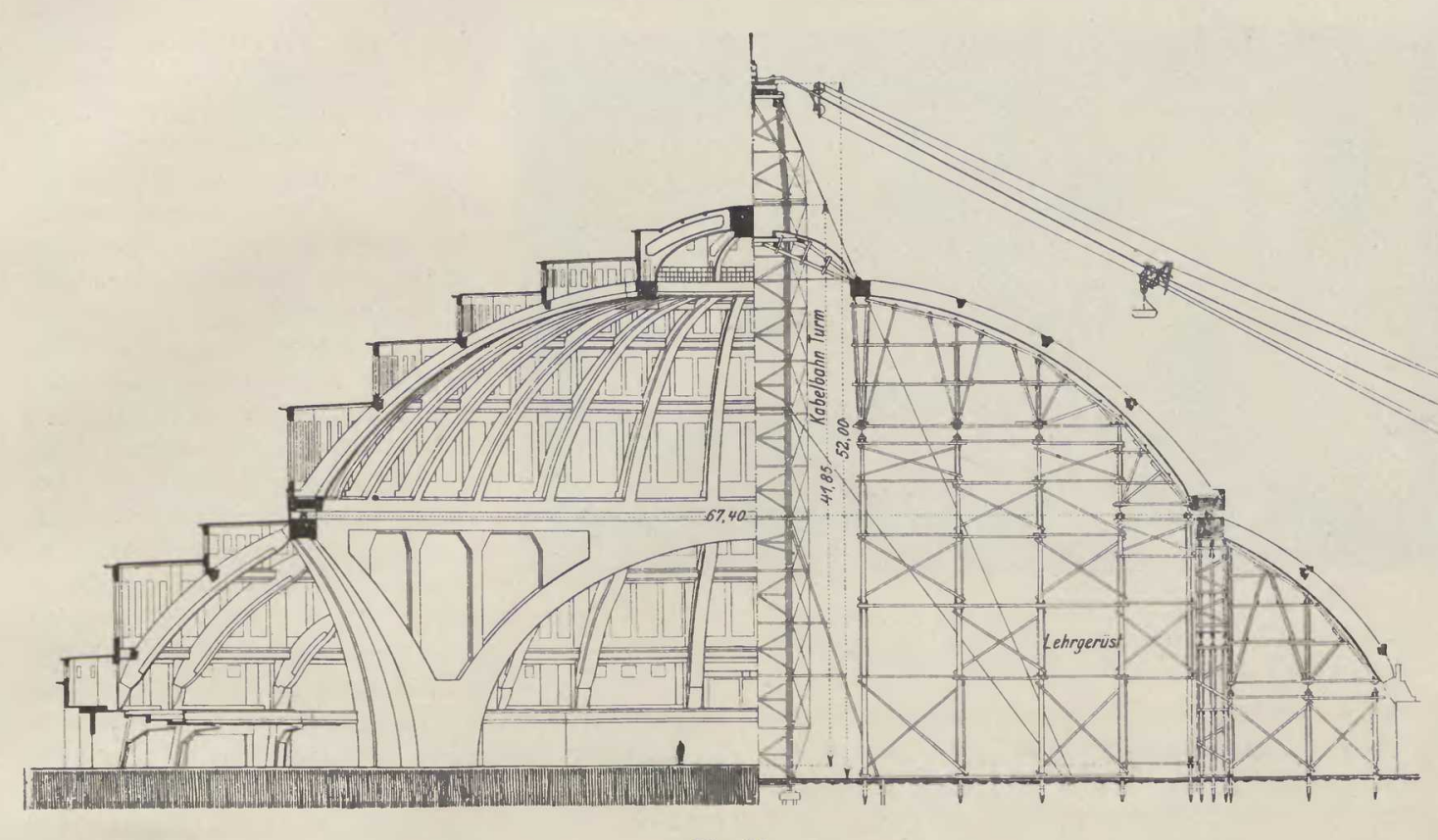

Iänsschnit durcll das Bauwerk mit Rüstun

bestimmter Stützung von der genannten Kuppellast lasten auf den Unterbau erreicht, wobei nur senkeine Seitenkraft von $0,55 \cdot 1200=660 \mathrm{t}$ für eine rechte und tangential gerichtete wagrechte Kräfte Apside aufzunelmen hatten. Zur weiteren Be- wirken konnten. Dagegen waren, abgesehen von urteilung der Größenordnung dieser Kräfte sei Reibung*), radial gerichtete wagrechte Kräfte ausnoch angeführt, daß̧ der Kämpferdruck der Haupt- geschlossen, die ungünstig auf den Unterbau bogen $1240 \mathrm{t}$ betrug. des Unterbaues und den orefen Lasten, die auf sung die 32 Rippen vollkommen gleichartig, so ihn wirken, wurde auch von der ausführenden Firma daß die günstigste Materialverteilung in der Kuppe der von Anfang an von der Bauverwaltung ver- erreicht wurde. Infolge dieser Lagerung könne

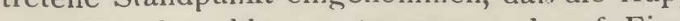
lager zu stellen sei.

Die andere Lösung lätte darin bestanden, an jeden der 4 Hauptpfeiler ein Rippenpaar von den Fundamenten ohne Unterbrechung bis zum Druck-
ring der Kuppel hinauf zu führen. Diese Lösung

T. Größe dieser Reibungskraft war durch einen Lagerversuch bereits genau studiert worden, der gelegent-
lich des Baues der Querbahnsteighalle Leipzig von den ausfibhrenden Firmen veranstalet worden war. (Vog
Gehler Deutsche Bauzeitung, Betonbeiblat 1912 Nr.9-11. Nach diesen Versuchsergebnissen war die Reibungskra 
sich die Spannungen aus den Wärmeänderungen wurde Eichenholz gewählt, um ein möglichst
durch die Verschiebung der Rollenlager zwangdurch die Verschiebung der Rollenlager zwang- ähnliches elastisches Verhalten wie bei Eisen-
los ausgleichen, während bei einer starren Ver- beton zu erzielen. Die verschiedenen Tragteile,
bindung der Kuppel mit dem Unterbau sehr un- die Pfeiler, die Strebebogen und die Aufbauten klare Beanspruchungen desselben entstehen wür- waren in einzelnen Stücken lose zusammengebaut, den. Endlich bot die Trennung der Kuppel vom ebenso war der Hauptbogen aus einzelnen Teilen Unterbau für die Ausführung den großen Vorteil, zusammengesetzt. Durch Blaupapier, das in die ausgeführt und auch ausgerüstet werden konnte. Dadurch wurde ein klares Bauprogramm und eine rasche Ausführung ermöglicht, also auch eine Ver-
ringerung der Kosten erreicht.

stetig wachsenden Belastung festzustellen. An diesem Modell konnte unter anderem der starke Einfluß eines etwaigen Ausweichens der

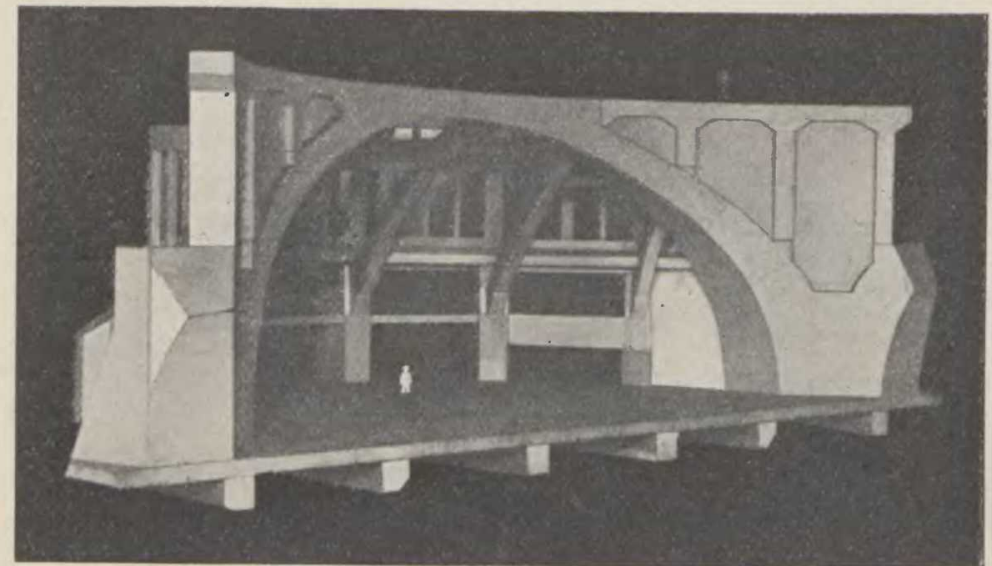

Holzmodell Fix. $_{\text {. }}$ Ve. ur Erkenntnis der Notwendig-

keit führte, die Abstützung durch
die Strebebogen so sicher als

irgend möglich auszubilden.

Gründungskeörper der Pfeich die

denen die Strebebogen ruhen,

besonders sorgfältig durchge

bildet, weil im Falle eines Kip-

pens dieser Pfeiler in der Bo-

daselbst entstandes wäre und dann die gesamte Abstützung
mittels der am oberen und unStren Ende gelenkig gelagerten bogen gewirkt, also keine hin-
reichende Sicherheit geboten hätte. Zur Vermeidung solcher anderungen wurden fernerverSo notwendig und schätzenswert aber die
theoretische Beleuchtung und Durchdringung
eines verwickelten statischen Problems auch ist eines verwickelten statischen Problems auch ist,
so wertvoll ist anderseits die Durchführung eines Vorversuches, sei es, daßs man einen wichtigen bis zum Bruch belastet, wie z. B. den Probebinder von $35 \mathrm{~m}$ Stützweite für die Querbahnsteighalle
des Hauptbahnhofes Leipzig*), oder aber, daß3 des Hauptbahnhofes Leipzig*), oder aber, daß
man ein Modell in verkleinertem Maßstab für ein größeres Stück des Bauwerkes herstellt und eben-
falls belastet. Ein solcher Modellversuch gibt
immerhin einen Anhalt für das Krättespiel und immerhin einen Anhalt für das Kräftespiel und
die Formänderungen, die vor allem auch beiStützensenkungen und Widerlagerverschiebungen einwurde daher ein Modell im Marsstab $1: 25$ nach Fig. 66 für eine Apside angefertigt und an den
Stellen der Kuppellager mit einer Gesamtlast von $6000 \mathrm{~kg}$ belastet, die dem oben erwähnten Wert von $6 \times 200=1200 \mathrm{t}$ entspricht, also $1 / 200$ der wirk-
lichen Last betrug. Als Material für das Modell Nr. 9 ) Siehe Deutsche Bauzeitung 1912 Betonbeiblatt
11. angeordnet (siehe Fig. 65) und zWar Band E zur Verbindung der Pfeilerköpfe, ein mittleres und oberes Band $\mathrm{D}$ und $\mathrm{C}$ am unteren gekuppelt, daß bei einem etwaigen Ausweichen
ines derselben, die anderen zur Lastübertragung

noch die Frage eingehend Gelenke eingefügt werden sollter Hauptbogen weifellos den Vorteil geboten, daß in dem stehenden Zylinderkörper von $200 \mathrm{~m}$ Umfangslänge, der mensetzt, Furen mit einem größten Abstand von $40 \mathrm{~m}$ ausgebildet worden wären, die bei starker Warmewirkung eine zwanglose Bewegung ermöglicht hätten. Durch solche Fugen wäre aber die aufgehoben worden und damit den Kämpfern rägerartige Wirkung eines jeden Bogens im Grundriß, die neben der Abstutzung durch die Strebebogen einen sehr willkommenen Sicher-
heitsfaktor bietet. Außserdem hätte aber die An- ordnung von Kämpfergelenken eine Zusammenziehung des Kraftstromes, der sich jetzt über einen Gelenkpunkt bedingt, wobei auch noch die Schwierigkeit der konstruktiven Ausbildung eines Kugelgelenkes für den ungewöhnlich grösen Druck von 120 t
wäre. ware.
Endlich ist aus Fig. 66 auch ohne weiteres zu
erkennen, daß̉ durch die Einfügung von Kämpfergelenken der vom Architekten gegebenen Bogengestalt kunstich Grewalt angetan worden wäre. Die Beobachtungen der Formanderungen in Einspannung der Bogen an den Kämpfern dringend
erwünscht war, so daßs man auf eine Einlegung von Kämpfergelenken verzichtete.

The Tragwerk der Kuppel aus den 32 Rippen, dem Zugring und dem Druckring gebildet wird, bestand doch die Notwendig-
keit, auch hier, älnlich wie bei den Strebeboge der Apsiden, die Aussteifung durch die wage rechten Ringdechen molichs wirksam zu ge-
stalten. Da das Ideal aller massiven Kuppel sicherlich immer die Vollkuppel ist, muß be jeder Rippenkuppel für eine solche wage-
rechte Aussteifung Sorge getragen werden, um rechte Aussteifung Sorge getragen werden, un
bei einseitigen Belastungen möglichst viele Rippen bei einseitigen Belastungen mogglichst viele Rippen
zur Mitarbeit heranzuziehen. An die Stelle der gekreuzten Diagonalen, die bei eisernen Kuppeln zwischen den Rippen und den Ringen liegen, treten bei den Eisenbetonkuppeln Vierecksgefache, deri Ecken moghichst steir, an den jeweils möglichst kräftige Ringe unter de senkrechten Wänden der Aufbauten ausgebilde und auf ihre Verbindung mit den Rippen beso-

(a) Schneelast einer Verdrehung desselben, vorz beugen. Als Tragwerk der Laterne wurden dahe 4 steife Rahmenbinder gewalit and der Anschlo wagerechten Vouten ausgebildet. Zum Schluß dieser Vorerörterungen sei nochmals der Grundgedanke der konstruktiven staltung hervorgehoben, nammlich das Besteben, dieses Raumgebildes zu erzielen. Die Eigenart der Lösung unserer Aufgabe geht besonders aus einem Vergleich mit einem anderen bemerken werten Ausfuhrungstalle, der Qherbahnsteighal gesetzte Bestreben vorlag. Der Grund bestandarin, daß beim Haupthahnhof Leipzig sehr ungünstige Bodenverhältnisse und $z$ war verschiede preßbarer Baugrund vorhanden war, die Haupt-
tragtenle zum Teil auf eigenen Füßen standen um Teil aber auch auf Mauerwerk ruhten, wobe waren. Daher wurden bei diesem Bauwerk alle Haupttragteile statisch bestimmt ausgebildet und zwar entiveder als Dreigelenkbogen oder als Barken gestreckten rechteckigen Grundriß dieser Halle uch die gegebene Lösung.

Ganz anders lagen die Ventalmisse bei unserer (a)

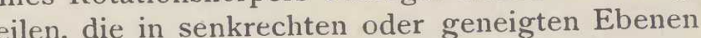
liegen, auch noch die Einfügung wagerechter, guer dazu liegender Glieder, durch die erst das

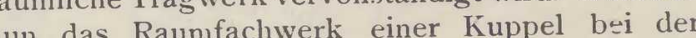

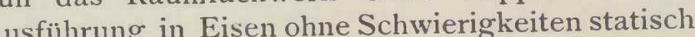
bestimmt ausgebildet werden kann, wird beim Eisenbetor schon durch die Eigenart seiner ErJung als Korper, der wie aus einem Gusse So erschwerend eine solche Vermehrung der statisch unbestimmten Größsen for die Berechnung ist, so vortelihaft wirkt sic

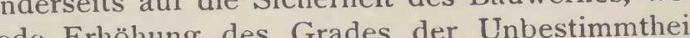
(je Veifokeit mit sich bringt. Der Eisenbeton ist daher für statisch unbestimmte Raumfachiwerke auf Grund der Eigenart seiner Erzeusungsweise besonders geeignet: Dircksicht anf die Erschwerniss 政 die Rechnung stets den Vorteil der weitgehenden Verteilung der einzelnen Lasten ausnutzen, weil sie ohne nennenswerte Kosten and oine Nur die eine Voraussetzung mufs hierbe gegeben sein, daß im Gegensatz zu dem Falle der Querbahnsteighalle Leipzig der Baugrun gleichmâsisg preßsbar ist, eine Forderung, die be it war.

B. Die konstruktive Gestaltung der Einzelteile und die Bauausführungen.

Die Einrichtung der Baustelle

Auf eine sorgfalttige und zweckmäßige Gewicht gelegt, weil der Bau in möglichst kurzer Zeit ausgefülrtrt werden sollte*)

*) Die Rekordleistungen der Amerikaner in der Ver
kürzung der Ausfiburumgrzzeiten bei groBen Bauten beruhe hauptsachlich auf einem klaren bauprouramm und einer
sorffiltigen Vorbereitung. Dadurch, dâ möglicht alle 


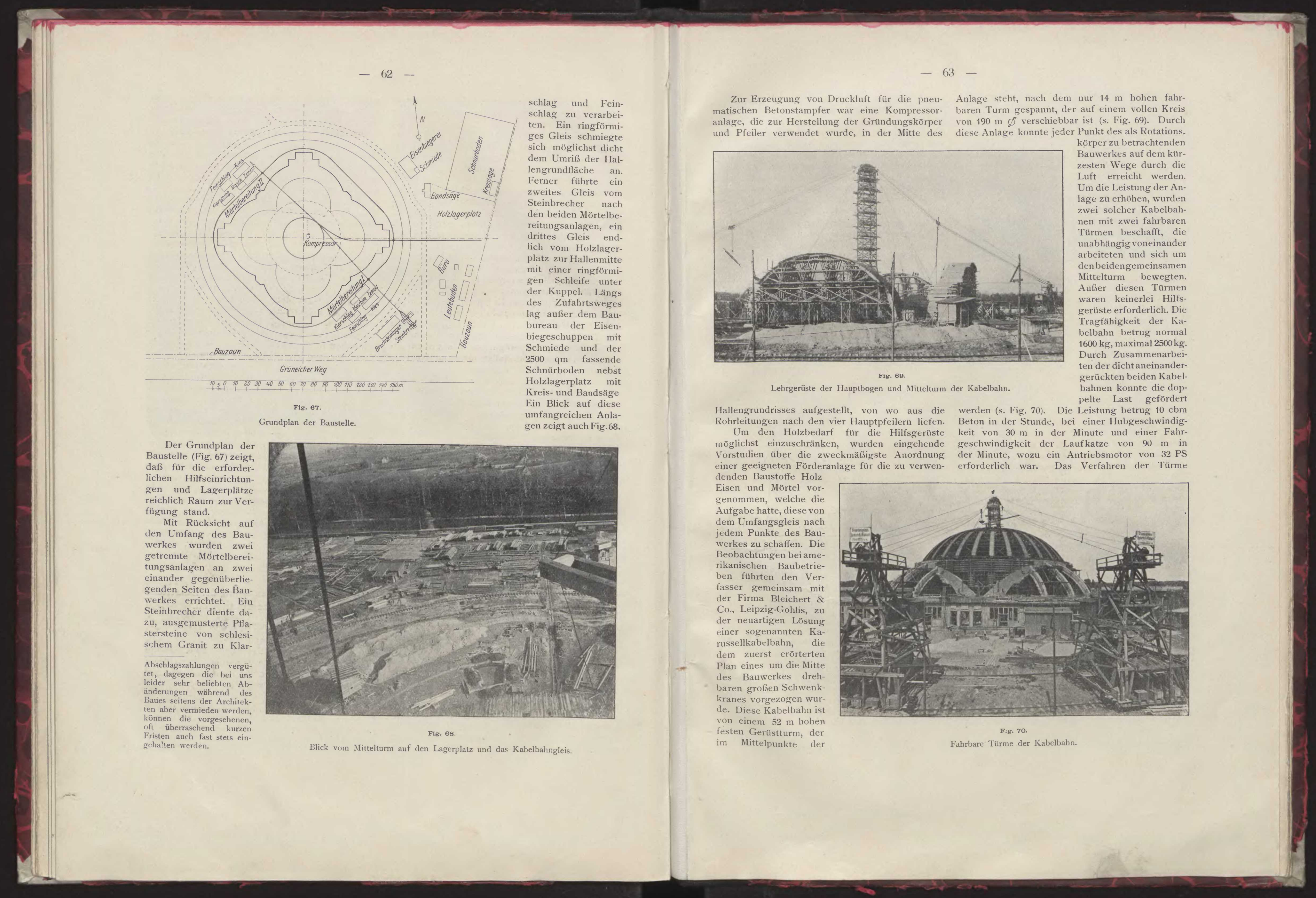




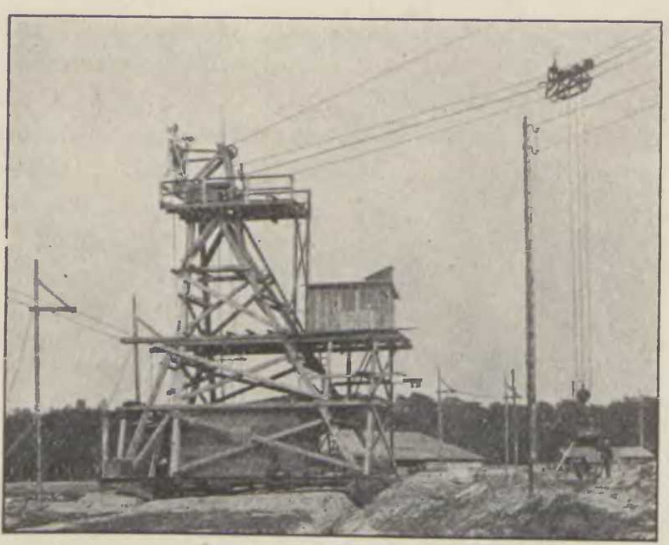

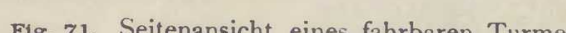
rfolgte mittels eines besonderen Motors, der im Nottalle durch wurde. Da die Höhe der fahrbaren Türme $14 \mathrm{~m}$, die des Mittelurmes aber $52 \mathrm{~m}$ und die Spannweite des
Kabels rund $100 \mathrm{~m}$ betrug, ergab sich die auß̧ergewöhnlich starke Neigung der $1: 3$. In Verbindung mitderunumgänglich nötigen groß̧en Fördergeschwindigkeit gabe für die Fir Bleichert \& Co. ge-
stellt, die hier wohl

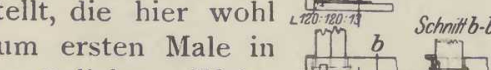
vorzüglicher Weise $\left.\frac{100}{0}, 1\right]$ gelöst wurde. Der Kabelbahn warin den beiden fahrbaren Türuntere Plattform dieser Türme wurde durch ein eisernes Gerippe gebildet, so
dars die Motoren auf inem steiten Boden befestigt werden konnten und die Antriebsvorrichtung keinen nachteiligen For-
menänderungen und Störungenauscesetzt war. Dagegen wurden die Auf bauten der Türme
mit dem Führerbäuschen aus wirtschaftichen Gründen in Holz ausgeführt. Das Traghe der Kabelbahn war mittels eines Betongewichtes auf dem fahrbaren Turm verankert. Von de Beweocung der Last auf ischen aus konite jede dem Auge verfolgt werden, wobi duche Ziger die genaue Einstellung erleichtert wurde. A diese Weise war zur Bedienung einer Kabelbah Das Gleis für die fat

Das Gleis für die fahrbaren Türme hatt die äußere Schiene lotrecht stand, war wie inher Schiene der Richtung der Druckstrebe des Turmes entsprechend geneigt, so daß sie auf einem Keget-

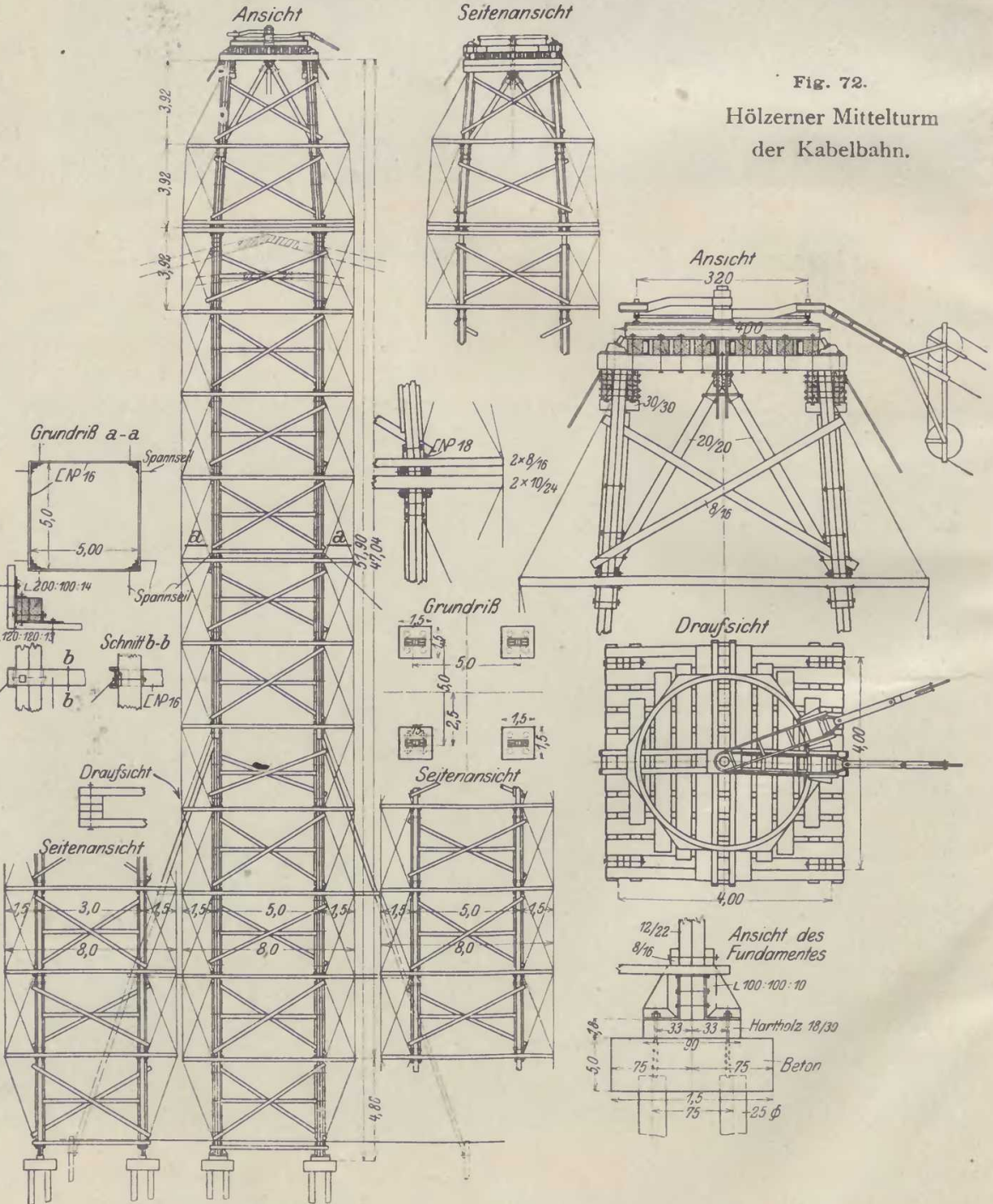

Schienen verwendet wurden, konnte die doppette Knoten des darüber und darunter liegenden Stockauf den

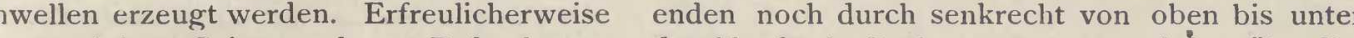
traf die von einigen Seiten gelegte Befürchtung, durchlaufende Drähte zusammengefaß̉st. Überdies daß die innere Schiene unbedingt auf einem durch-
laufenden Betonklotz liegen müsse, um störende
Stockwerken durch wagerechte Riegel verringert

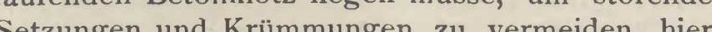
Setzungen und Krümmungen $\mathrm{zu}$ vermeiden, hier
nicht $\mathrm{zu}$, obwohl die Schwellen zum Teil sogar in aufgeschüttetem Boden gelagert waren. An der
Außsenseite des Gleises war Außenseite des Gleises war
die Schleifleitung für die
Stromzufuhr auf hölzernen Masten verlegt.

Die konstruktive Ausbildung des Mittelturmes zugang für die Hilfsge-
zugeition als Haupt rüste diente, war des
wegen eine eigenartige

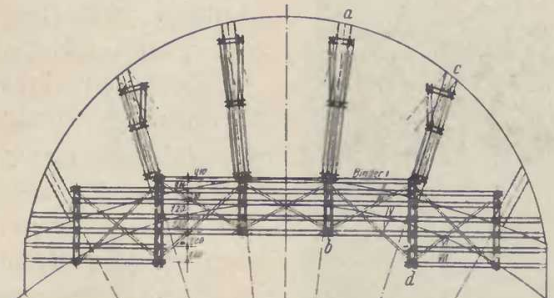
seilen oder dünnen Dräht ten die Gefahr besteht, Holz einschneiden, sind hier für den Angriff der Drähte wagerecht liegende eiserne Rahmen angeordam Kopfe und in halber Höhe umfassen (siehe
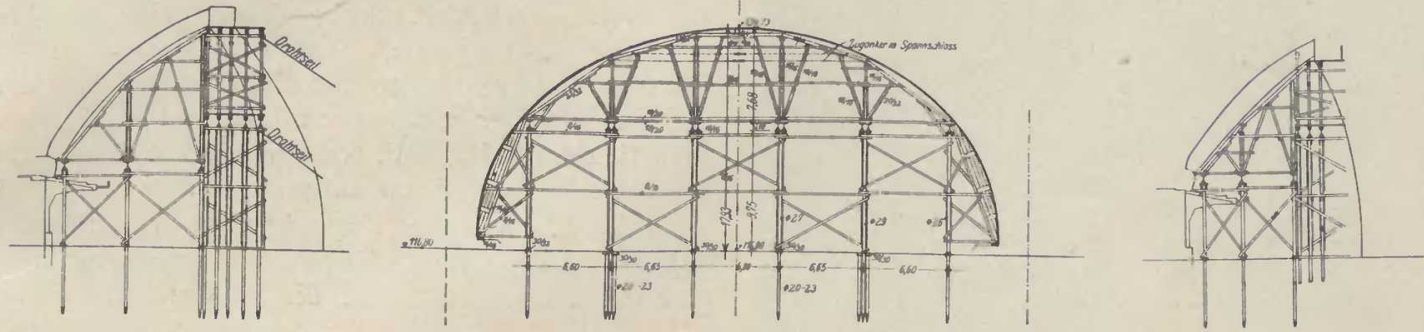

Fis. 73

Lehrgerüste des Hauptbogen.

Autgabe, weil in einer Höhe von $52 \mathrm{~m}$ eine loses Drehen der beiden Kabelbahnen un wagerechte Kraft von $20 \mathrm{t}$ angrih, die durch den Konigszapfen zu ermoglichen und eine klare Theron Turmes in Eisen wäre wohl konstruktiv einfacher lagen bestehenten Plattform ein Schienenkranhohen Kostenaufwand, weil dieses eigenartige wagen abgestützt war. In dem Bauzustand der (a) daher dieser Turm in Holz ausceführt, trotz der hat er wäbrema der O jtenth 1912 einen orkaSchwierigkeit, die vielen Storstellen einwandfrei artigen Sturm ohne jeden Schaden überstanden. Einer Oberlastung des Turmes bei den vieauf eingerammten Holzparmes sind nach Fig. 2 fach wechselnden Belastungen durch die Kabefachwerkartig miteinander fest verbunden. Sie Gegengewichtes in bestehen aus je zwei Kanthölzern, die mit ab- beugt; dieses war so gewählt, daß der Turm sich wechselnden Stôsen durch die 13 Stockwerke ge- von der äußeren Schiene abhob, sobald eine Überführt sind. Am Kopfe des Turmes und in halber schreitung der zulassigen Belastung der Kabestämme im Anfang des Baues wurde wiederho spannt. Um die Knicksicherheit zu erhöhen und ein solches Abheben bis zu $0,5 \mathrm{~m}$ beobachtet und vor allem die Stobstellen einwandfrei zu stutzen, dadurch der Kranfuhrer zur Vorsicht gemahnt. hinaus verlägert 


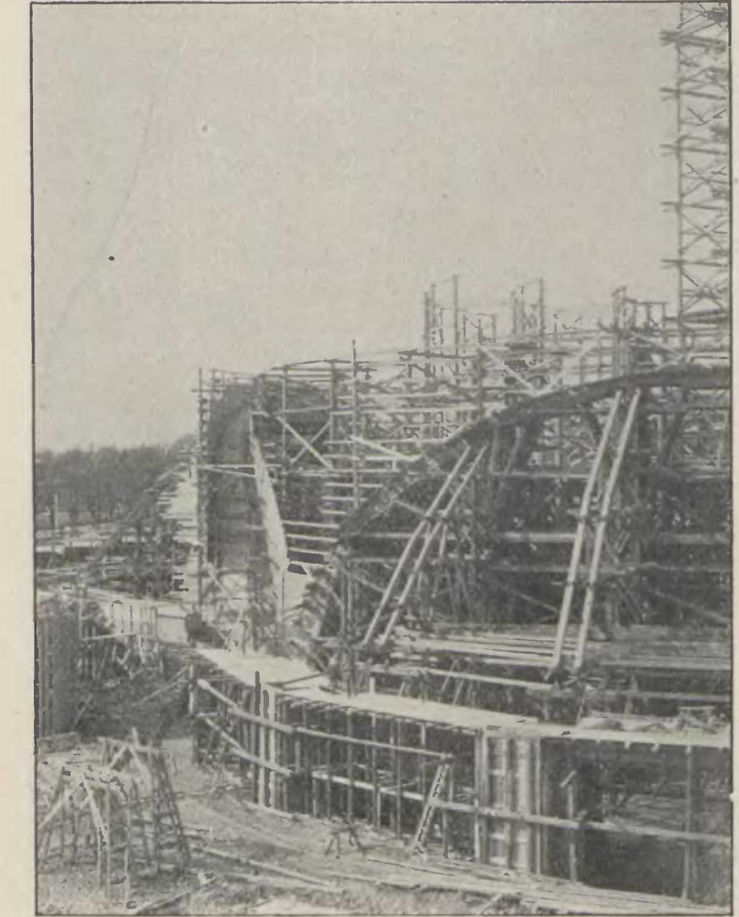

Fig. 74

Gerüste und Sclalung der Hopthogen und Strebebogen,

sowie des Daches.

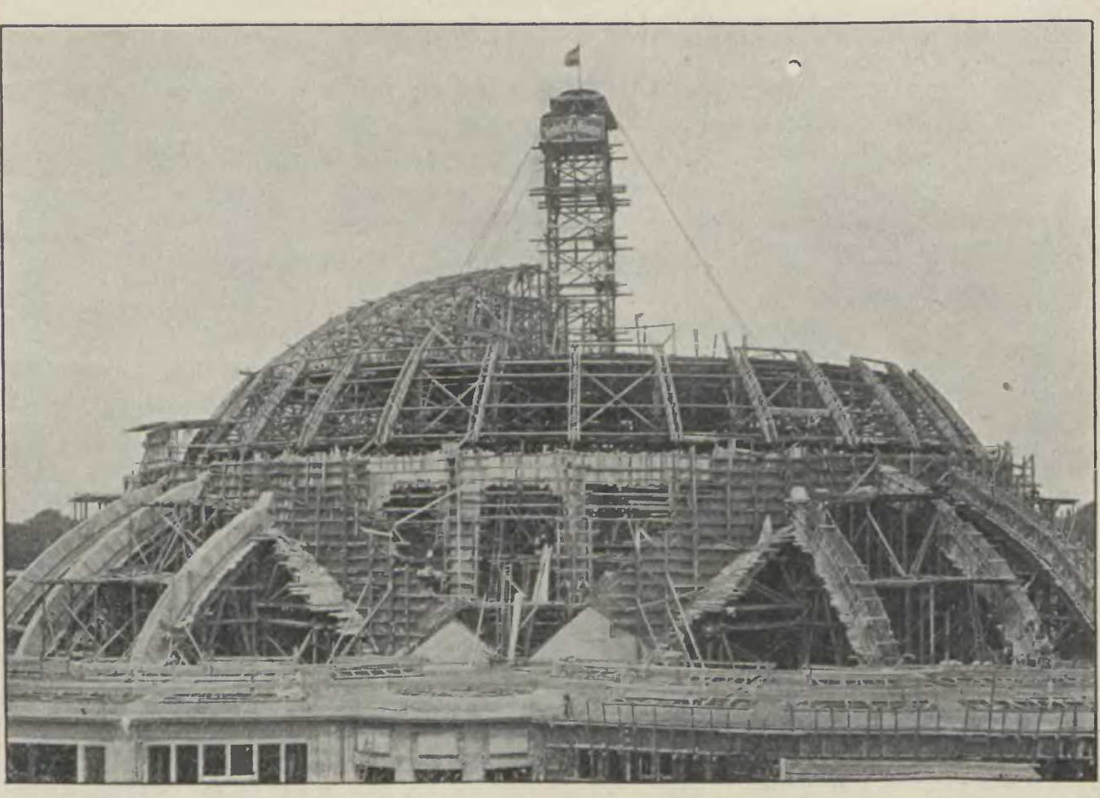

Fix. 75

üstung der Kuppel. rechten Seilzuges und somit ein willkommener Diese neuartige Anlage, die von der Firma
a Bleichert \& Co. in der maschinellen Einrichtung ausgebildet wurde, hat sich in jeder Hinsicht bewährt und wesentlich dazu beigetragen, die Ausdie Anwendung dieser Anlage war allering fur die Baupolizei, abweichend von der sonst üblichen Auffassung, die Anwendung eines Hebezeuges gestattete, bei dem die Lasten über die arbeiterden sei darauf hingewiesen, daß Sonders sei darauf lingeanesen, daß irgendei Die Rüstung für die 4 Hauptbogen (siehe Fig. 69 u. 73 ) ist in bekannter Weise durchgebildet. Bemerkt sei hierbei, dais die einzelnen Bin-
der im Grundrißs trotz der Krümmung des Bauwerkes eben ausgebildet waren, wobei nur ${ }^{2}$ übrigen nur so weit, als es die Schalung erforderte. Die Rüstung für die Strebebogen diente gleichzeitig gur seilliche. Die Joche des Unterbaus dienter Rüstung sowie die großsen Schalungsflächen fü die Hauptpfeiler wurden an Ort und Stelle, au dem Erdboden liegend, zusammengebaut und in ganzen mittels Winden aufgerichtet.

Das $37 \mathrm{~m}$ hohe Kuppelgerüst bestand der Rippenteilu Gerüstwänden, die im Grundrif radia angeordnet waren un in üblicher Weise ausge Die Gerüstständer wig. den auf Holzpfählen gegründet. Bemerkenswert ist, daß die $5 \times 32=$ 160 Stück eisernen Ge
rüstspindeln wegen der starken Krümmung der Kuppel in zwei Stock-
werken angeordnet wa*) Bekanntlich sind die die großzaigige Eininichtung
ihrer Baustellen und die Aus bildung und Hend Handhabung
ihrer Hebezeuge uns voraus ihrer Hebezeuge uns vorauc.
Dieser Vorsprung kann nur dann eingeholt werden, went
auch die Behörden die Arbeiterschutzestimesten mungen in möglichst wenig eng
herziger Weise anwende Iragen, unsere Arbeiter grö̉erer Achlisambeit un
zu selbständiger Beurteilun etwaigstarn Gefiger Beurteilung
ziehen. ren, in denen auch eine vollständig dichte $\mathrm{Ab}$ deckung der Arbeitsfläche in zwei Ringen zum
Schutze der Arbeiter vorhanden war. Die Laternenrüstung wurde später auf den fertigen Druckring der Kuppel aufgesetzt, so daßs später das Kuppelgerüst unabhangig von der Ausfuhrung der Laterne entfernt werden konnte. ogen mit ihrer eigene Lehrerïste für die Haupt und Strebebogen im fertigen Zustande und der Beginn des Aufbaules der Kuppelrüstung mit den Lehren für den Kuppelunterbau. Die nahezu fertige Kuppelrüstung zeigt Fig. 75, in welcher der Hauptbogen und des Windrahmens deutlich $\mathrm{zu}$ erkennen ist.

C. Die Ausführung des Unterbaues.

Der Baugrund bestand allenthalben aus gleichmaasig Sestgelagertem Sand, sodais ein gleichmaalsiBauteile zu erwarten war. Die Bodenfuge der Pfeiler war übrigens nicht wagrecht, sondern geneigt angenommen und $z$ war möglichst rechtwinklig zut die Hers dil erfolgte in bekannter Weise unter Absenkung des Grundwassers mittels Rohrbrunnen. Bei den Hauptpfeilern waren diese Rohrbrunnen nur entmöglichst in der Nähe der tiefsten Stellen der Baugrube angeordnet und zwar jeweils 3 Stïck mit etwa $5 \mathrm{~m}$ Abstand voneinander.

Der Kiesbeton für die Gründungskörper im unteren Teile hat das Mischungsverlältnis Einbettung der Eiseneinlagen $1: 4$ und bei den Pfeilern ein Klarschlagbeton 1:6:8 verwendet wurde. Für sämtliche GründungsPfeiler un fur die nur schwach bewelirten großen stampft, wobei für einen Stampfarbeiter zum Heranbringen und Verteilen des Mörtels 5 Hilfsarbeiter notig waren. Durch Versuche konnte
hierbei festgestellt werden, daß 3 , nachdem hierbei festgestellt werden, daß, nachdem
der Beton von Hand mit der vorschriftsmäßigen Anzahl von Stampfstößen abgerammt und von $18 \mathrm{~cm}$ auf $15 \mathrm{~cm}$ Schichtstärke verdichtet war,
durch die nachträgliche Verwendung von Pre 3 durch die nachträgliche Verwendung von Preßßluftstampfern eine weitere Zusammendrückung
um $2 \mathrm{~cm}$, mitunter sogar um $3 \mathrm{~cm}$ erreicht um $2 \mathrm{~cm}$

*) Eingehende Vergleichsversuche über die Druck-
festigkeit von Betonkōrpern, die mit Preßluftstampfern

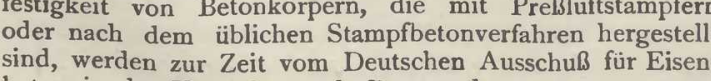

Für die großen armierten Betonkörper mit

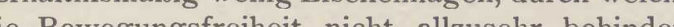

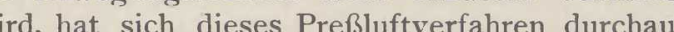
währt. Diese Erfahrung ist um so beachtenswerter, als auch im Betonbau, wie überall in Buuwesen, die gelernten Spezialarbeiter immer lener werden und wir, genau wie die Amer-

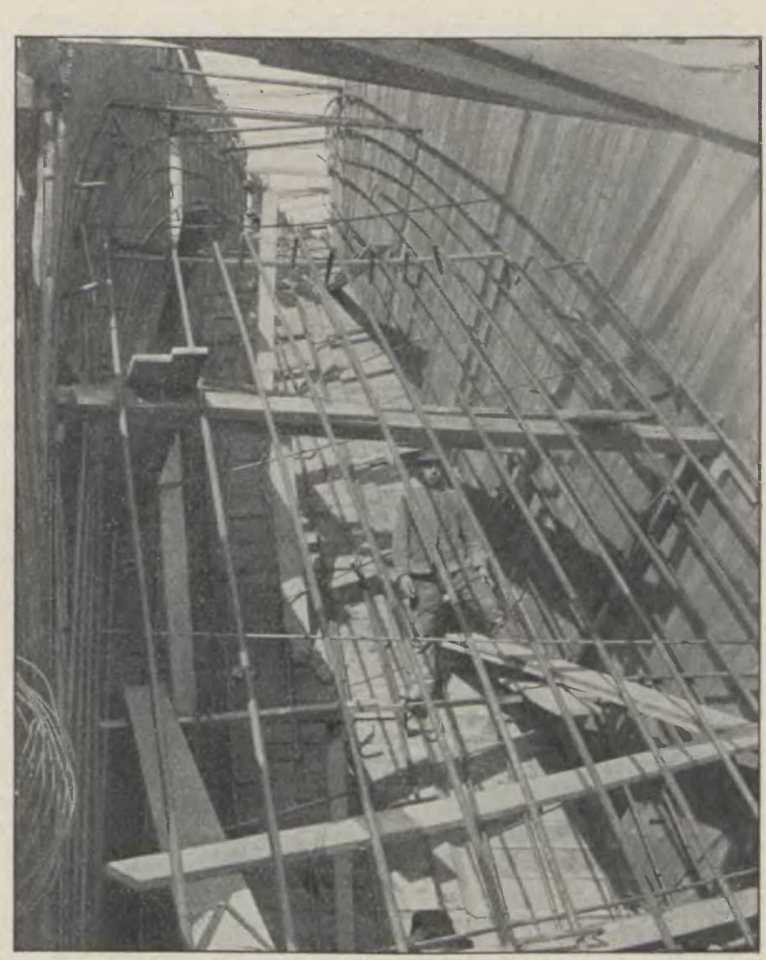

Schalung und Eiseneinlagen der Hauptbogen. Die Bewehrung der Hauptbögen ist in Fig. 21 er Abmessungen aus Fig. 76 deutlich hervorgeht. Da für die sämtlichen inneren Ansichtsachen des Bauwerkes keinerlei Verputz vorgeseten war, wurden die Schalbretter an den StinSpure des Bogens hervortreten zu lassen. Die Längseisen liefen parallel zu den 4 gekrümmten Flächen der Hauptbögen. Im Scheitel betrug die Beim Kämpfer bei $21,0 \mathrm{gm}$ Ouerschnitt nur $0,06 \%$ Diese verhältnismälig geringe Eisenbewehrung, die auf Grund der sorgfältigen Berechnung unter Berücksichtigung aller Nebeneinflusse (verg. 
daß für solche Druckglieder der Eisenbeton im vorzanglich geeigneter und auch durchaus wirt-
schaftlicher Baustoff ist. Mit Rücksicht auf die schaftlicher Baustoff ist. Mit Rücksicht auf die
allerdings verschwindend kleinen Torsionsspannungen (vergl. Seite 27) wurden noch einige Schrägeisen in den Bogenflächen eingelegt. D bei diesen aułsergewöhnlich großen Querschnitten
die üblichen Bügel nicht erforderlich waren, verankerte man die Eiseneinlagen an der inneren S-förmiger Bügel im Betonkörper, um ein Ab-

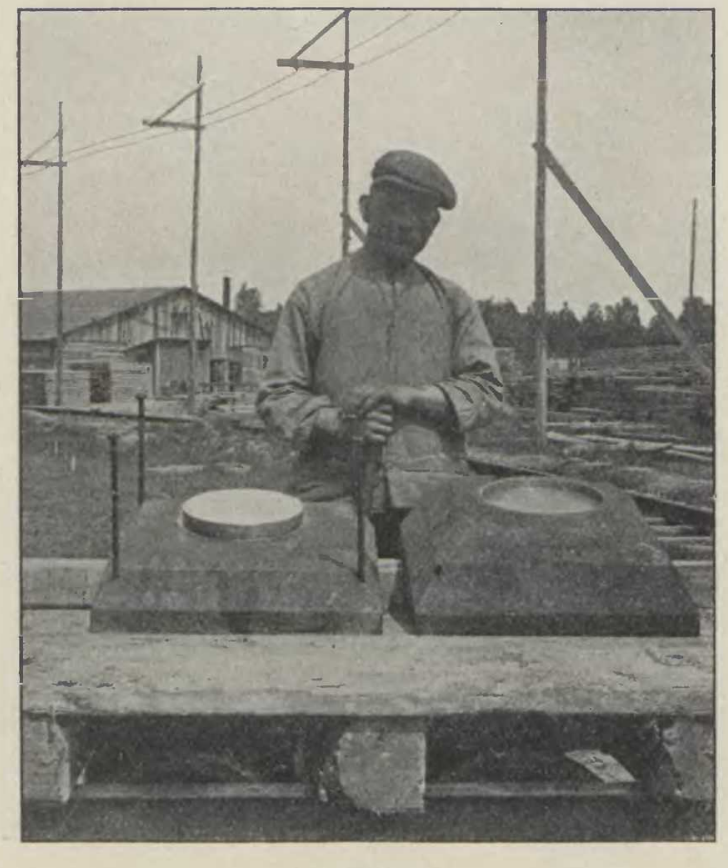

Flg. 77.
Lagerschalen fur die Gelenke der Strebebogen

springen der
zu verhindern.

Das Mischungsverhältnis des Betons zur Einbettung der Eiseneinlagen an den 4 Bogenflächen
war 1 Teil Zement, 3 Teile Kiessand und 3 Teile Granitfeinschlag, für den inneren Betonkern ohne
Eiseneinlagen dagegen (vergl. Fig. 21 Seite 28) Eiseneinlagen dagegen (vergl. Fig. 21 Seite 28)
den auftretenden Beanspruchungen entsprechen im unteren Teil $1: 6: 8$, im mittleren Teile $1: 5: 61$ und im oberen, ${ }^{2}$ Seile $1: 4: 51 / 2$, endilich für $\mathrm{d}$ $1: 21 / 2: 21 / 2$ und daran anschließsend auf $2,5 \mathrm{~m}$ Länge
$1: 3: 3$. Das Betonieren erfolgte in der bekannten Streifenteilung, wie es allgemein bei gewölbten Betonbrücken üblich ist, um dem Schwinden des
Betons Rechnung zu tragen. Zur Erörterung

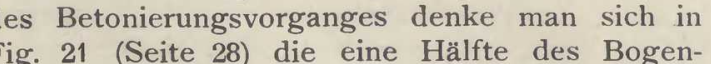
(Serte 28) die eine Halte des Bogen-

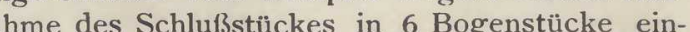
geteilt und in dieser Reihenfolge beziffert. Zuerst wurden der Reihe nach die Streifen 2, 4 und 6, sodann 1,3 und 5 ausgefuhrt, endlich zuletzt das war darauf Rücksicht genommen, ausgeführten geradzahligen Streifen möglichst senkrecht über den lotrechten Stützen der Lehrgerüste lagen und somit ein möglichst frühzeiDie für die Ausführung bedeutsameinatren. eiten der Strebebogen in den Apsiden sind in Fig. 22 und 24 (Seite 29 und 30) $\mathrm{zu}$ sehen. Nachdem die senkrechten Pfeiler im Umfang der Apsiden fertiggestellt waren, folgte Unnachst die Gen untenken für die Strebebogen. Mittels eines Holzkastens wurde der Raum für diese Kugelgelenke ausgespart und das Gelenk, welches aus zwei Stahlgusschalen (siehe Fig. 77) von der Seite her eingescho Seite 29) erkennbare $2 \mathrm{~cm}$ starke Fuge rings um die Kugellagerschalen wurde mittels einer $5 \mathrm{~cm}$ starken Gipsleiste langs ihres Umfanges abgeden auf diese einstweilige, leicht wieder $\mathrm{zu}$ beseitigende Füllungsmasse der Fuge ein Streifen aus Teerpappe gelegt, um ein Anhaften des aufzu-

Die an diese Lagerplatte aus Stahlguß anschließenden Eisenbetonkörper wurden mit drei Lagen gekreuzter Eisen von $12 \mathrm{~mm} \varnothing$ und $5 \mathrm{~cm}$ laschenweite bewehrt, bei einem MischungsErgebnisse von Druckversuchen seien hier angewhrt. Die Festigkeit der Betonwürfel von $30 \mathrm{~cm}$ Kantenlänge betrug nach 28 Tagen $260 \mathrm{~kg} / \mathrm{qcm}$, die ganze Fläche übertragen wurde. Schränkte man lagegen die Druckfläche auf den vierten Teil, so erhöhte sich die auf die Flächeneinheit dieser kleineren gedrückten Fläche bezogene Bruchbe-

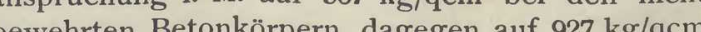
ach ebenfalls 28 Tagen bei den Körpern die in der bei unserem Bauwerk vorgesehenen Weise mit Eisen bewehrt waren. Nach einem Jahre stieg diese Zahl sogar auf $1089 \mathrm{~kg} / \mathrm{qcm}$, so daß man durfte. Diese Vorversuche bestätigen die Ergebisse, wie sie bereits beim Bau der König-FriedrichAugust-Brücke in Dresden und der Querbahn- steighalle in Leipzig von der Firma Dyckerhoff \& Widmann A.-G. festgestellt sind.

Um ein Verschieben der Gelenke vor der Ausrüstung zu verhinden, waren, wie Fig. 77 zeigt, vor der Ausrüstung abgeschnitten wurden.

Während bei unserem Bauwerk im allgemeine mit einem verhalltnismäß乃ig geringen Wasserzusatz
gearbeitet wurde, mußte bei den Strebebogen gearbeitet wus, mubte bei den Strebebogen feuchterem Zustande verarbeitet werden, weil wegen der beiderseits geschlossenen Schalkästen
das Einbringen und Verarbeiten des Mörtels nur das Einbringen und Verarbeiten des Mörtels nur
von oben her erfolgen konnte. Die Ausführung von oben her erfolgen konnte. Die Ausführung
wurde im unteren Teil der Strebebogen, etwa bis $\mathrm{zu} 2 / 3$ der Bogenlänge, noch dadurch erschwert, daß hier auch die obere Rückenfläche im Verlauf des Betonierens, ähnlich, wie es in der Regel bei
den Säulen geschieht, allmählich zugeschalt werden den Säulen geschieht, allmählich zugeschalt werden
mußte und dadurch ein sorgfältiges Unterstopfen
erford.rlich wurde. Der obere Teil des Kopfgelenkes war übrigens bereits bei der Herstellung der Hauptbogen ausgeführt worden.

Nach Fertigstellung der Strebebogen wurden die wagerechten Versteifungsbänder sowie die
Aufbauten über den Apsiden ausgeführt Einbindung der wagerecht liegenden Versteifungsbänder wurden bei der Ausführung der Strebebogen $2,5 \mathrm{~m}$ lange Eiseneinlagen mit einbetonier bogen eine der Querschnittsforn der Strebeangepaßste $5 \mathrm{~cm}$ tiefe Aussparung vorgesehen. Ferner waren für die später herzustellenden senkrechten Pfeiler der Apsidenaufbauten treppenförmige Die scharfe Trennung des Unterbaues von der
Kuppel erlaubte es, die Ausrüstung des Unterbaues
unabhängig von der Ausführung der Kuppel unabhängig von der Ausführung der Kuppel
vorzunehmen und sich so ein vollständig klares Bild über die Kraftwirkung während der einzelnen Bauzustände zu verschaffen. Ein weiterer Vorteil
lag aber auch in der Möglichkeit, die Rüstung rrülzeitig $z u$ beseitigen, dadurch die Räume der Apsiden für den Finbau der Tribünen und die weiteren Ausbauarbeiten zugänglich zu machen
und das Rüst- und Schalholz für den Bau der Kuppel weiter zu verwerten. Für die wiederholte Verwendung der Schalbretter während der ganzen Bauzeit wurde ubrigens eine Art Fahrplan derselben
aufgestellt und durchgeführt.

einzelnen Bauzustände erwies nun, daß es nicht zulässig war, das Tragwerk des Unterbaues auszurüsten, bevor die Aufbauten über den Apsiden als
günstige Auflast auf die Strebebogen wirksam wurden. Ebenso notwendig war es aber, nach Fertigstellung dieser Aufbauten auch noch den
Beanspruchungen der Hauptbogen vorzubeugen Diechdem die Aufbauten in den Apsiden und der Dring der Kuppel fertiggestellt waren.

Das Ausrüsten der Hauptbogen erfolgte derart gegenüber liegender Hauptbogen zum Teil ab政 Wipterion von allmälliche Absenkung des Gerüstes und eine moglichst gleichmälfige Beanspruchung

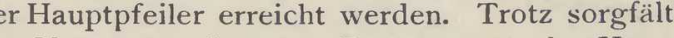
政 festrestellt werden
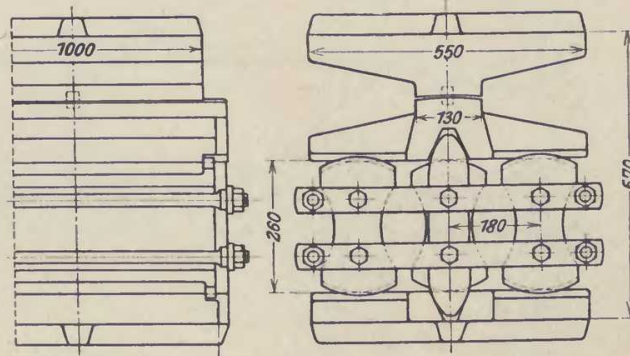

$-1050 \longrightarrow$

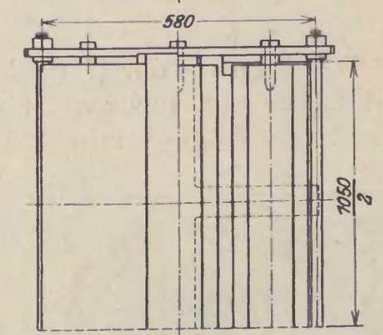

Die sogenannten Windrahmen, die das Trager Hauptbogen bilden, wurden mach Fertigstelung der Betonierungsarbeiten der Apsiden (sieh ig. 21, Seite 28) ausgeführt. Wahrend die

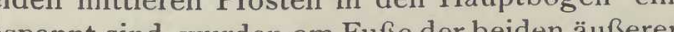
osten Gelenke ausgebildet. Die Formgebung leser Gelenke bei der Herstellung im Bauwerke erfolgte durch Einlegen von
ig. 21 , Seite 28), die entsprechend der vorgesehenen Krümmung des zylindrischen Wälzgelenkes Im äußeren Teil der Fuge in größserer Zahl, und zwar bis zu $4 \mathrm{~mm}$ Stärke übereinander gelegt
wurden. Der obere wagerecht durchlaufende Balken des Windrahmens war von den beiden nschließsenden, mit den Hauptbogen fest vergetrennt, die in einfachster Weise durch Anstrich mit Seifenlauge hergestellt wurden. 


\section{$-70$}

D. Die Ausfuihrung der Kuppel. Die 32 Kuppellager waren nach Fig. 78 ausgean den 32 Knickpunkten eines jeden Trägers je und Durchbildu, 56, Seite 51). Ihre Bemese be te Aufris und Grundris zu gewahrleisten, wurden bahnhof in Leipzig auf Grund der dort vorge- $\begin{aligned} & \text { vorher Betonklötze mit entsprechenden Ab- } \\ & \text { messungen hergestellt, eingebaut und später mit }\end{aligned}$
nommenen Versuche.

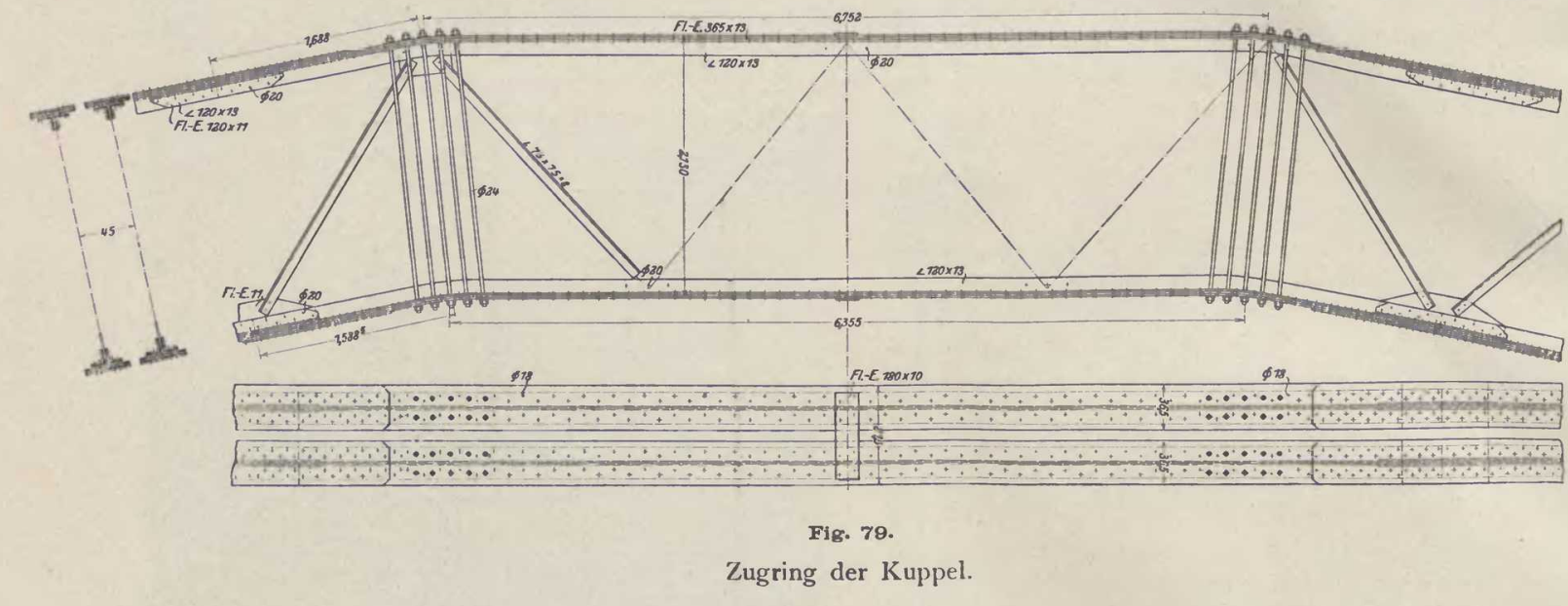

Der Zugring der Kuppel bestand, wie Fig. 79 einbetoniert. Zur Erzielung eines sicheren Verbur-

und 80 zeigen, aus zwei übereinander liegenden
genieteten Trägern mit Strebenfachwerk im Ge_-

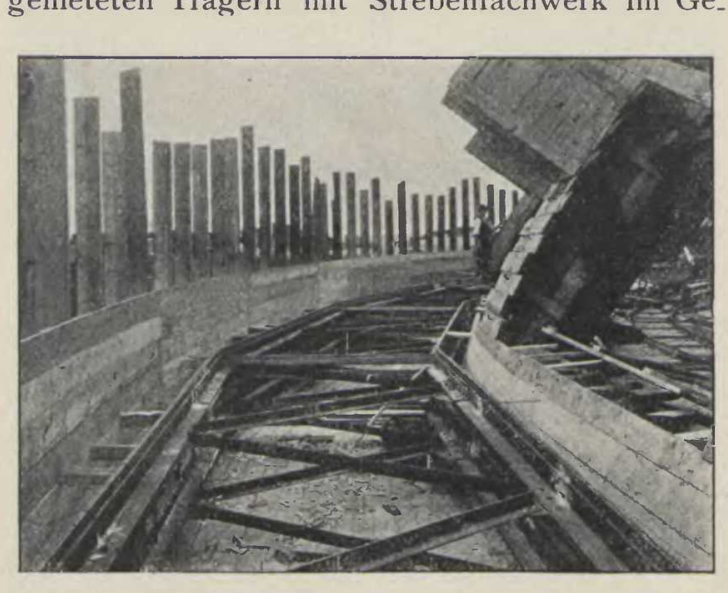

Fls. 80.
Montage des 7 .

samtgewicht von 130 t. Die Kosten dieser Eisenkonstruktion betrugen $34000 \mathrm{M}$. Auf die sorgfämaßen das Widerlager der Kuppel bildet und vo den Windkräften auch auf Biegung beansprucht Zugkraft betrug rd. $500 \mathrm{t}$ bei einer zulässige flächen der Gurte eingelegt worden.

Da das Betonieren des Zugringes vor dem er in 8 durch einfache Fugen getrente, wurde

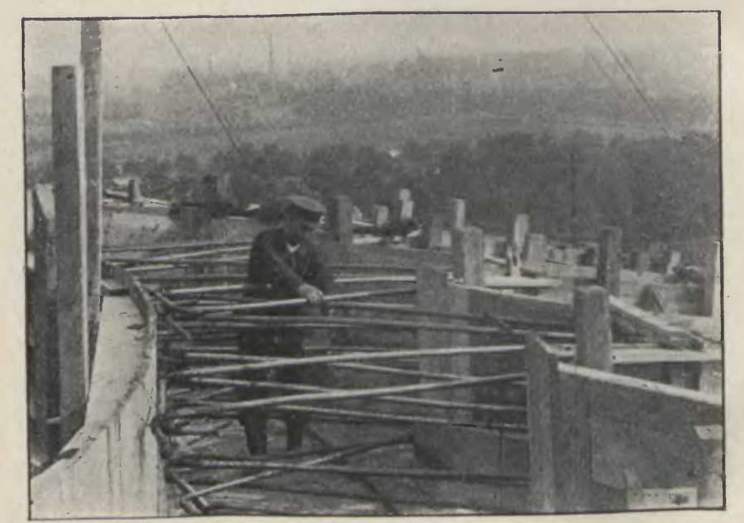

Fil. 81.
Eisenbewehrung des Druckringe.

stücken hergestellt, von diesen zuerst die 4 über Im Anschlußs hieran die übrigen dazwischen-

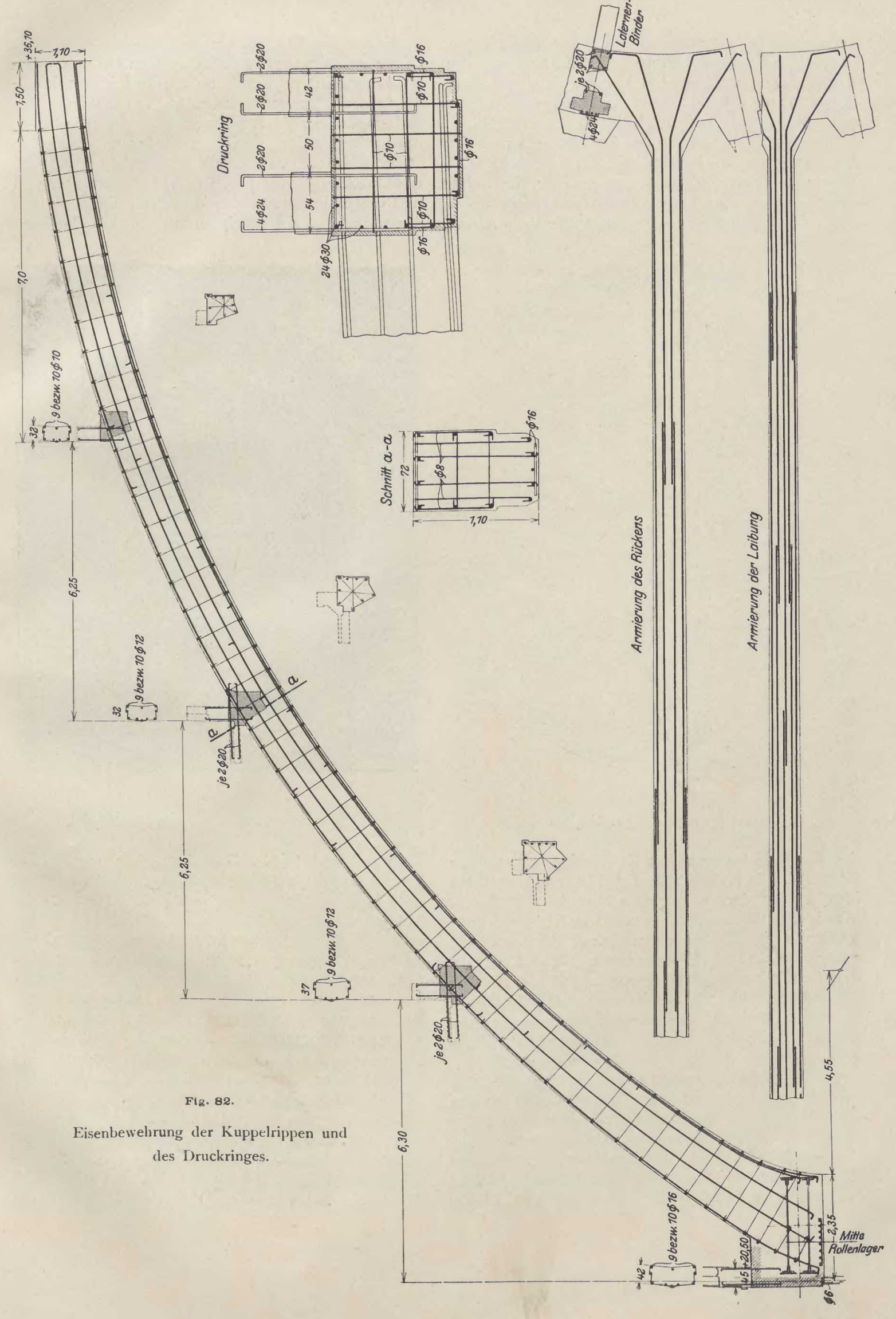




\section{$-72-$}

liegenden Teile betoniert. Gleichzeitig mit der gewichte des Tragwerkes der Kuppel entsprach. Herstellung des Zugringes erfolgte auch die Diese war in unserem Falle um so leichter Maßsder 32 Kuppelrippen bilden, so daß am Kämpfer führbar, als hierzu das vorzügliche Hilfsmittel der jeder Rippe eine radiale Arbeitsfuge entstand. Kabelbahn zur Verfügung stand. Mit ihrer Hilfe Die Eisenbewehrung des Druckringes und der tho mithels echer weiteren Motorwinde wurden Eisen dienten allenthalben schleifenförmige Bügel. ound die gleiche Last ringförmig im Grundriß aut Um eine möglichst zuverlässige steife Verbindung die einzelnen Kuppelrippen in $1 / 4$ und $3 / 4$ ihrer
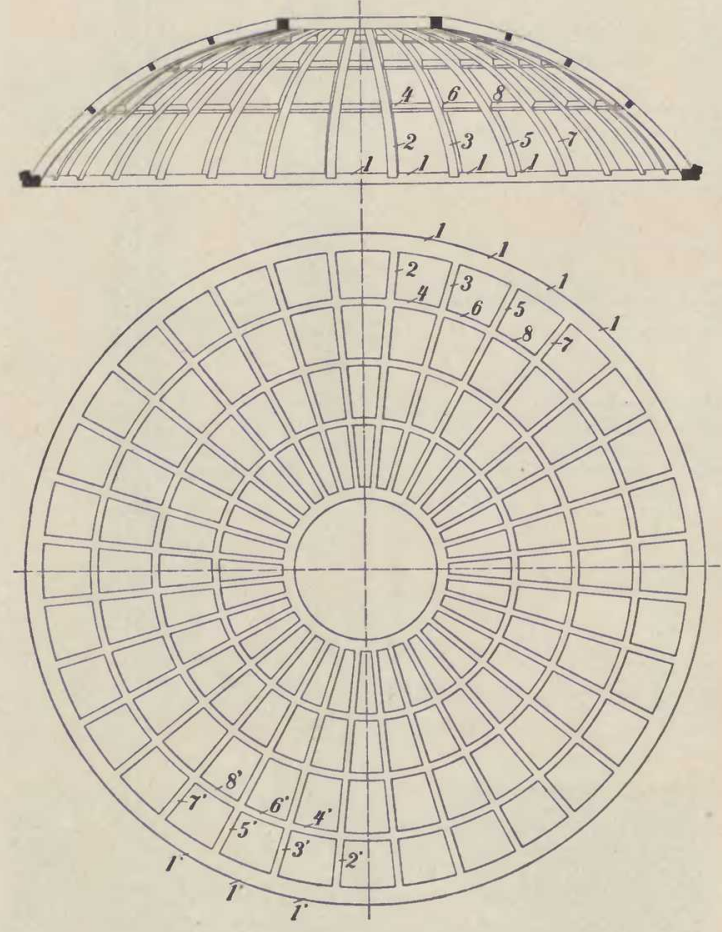

Betonierungsfolge der Kuppel.

des Druckringes mit den Kuppelrippen herbeizuinfolge einer Verdrehung desselben bei einseitiger Wind. und Schneelast vorzubeugen, sind diese an illrem oberen Ende im Grundriß voutenartig
verbreitert und die Eiseneinlagen fächerförmig in

Bei allen Großkonstruktionen aus Eisenbeton besteht bekanntlich die schwierigste und bedeutsamste Aufgabe für die Ausführung darin
während des Betonierens die Formänderunge des Lehrgertstes unter dem außergewohnlich ein Mindestmaß zu beschränken, um Zugrisse in dem jungen Beton $z u$ vermeiden. Aus diesem Grunde wurde hier zum Zusammendrücken Auflast aufgebracht, die dem späteren Eigen-

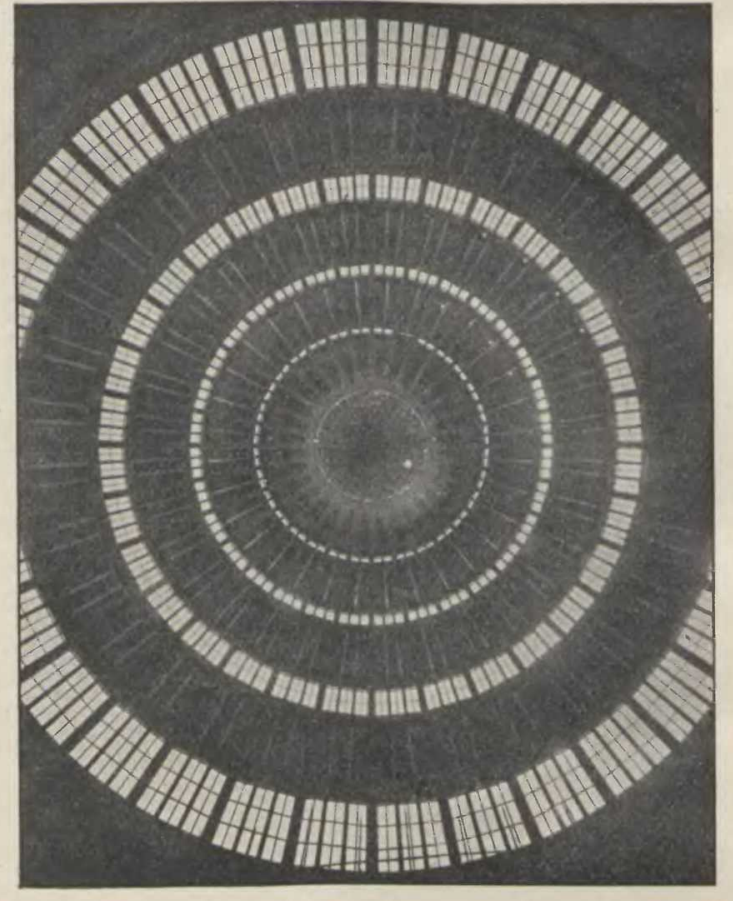

Fiz. 84.
Blick von unten nach oben in die Kuppel.
Stützweite verteilt. Das Aufziehen der Säcke

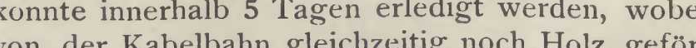
dert wurde. Die Kosten dieser Maß3nahme haben sich auf nur etwa $500 \mathrm{M}$. belaufen, walirend die günstige Wirkung der Zusammenpressung des Mit dem fortsclureitenden Betuieren der Kuppelrippen und Ringe ließ man sodann der Sand aus den Säcken auslaufen, so daß jeweils

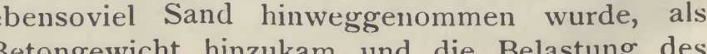
ehroerustes dauernd dieselbe blieb. Der Vorgang beim Betonieren der Kuppel war
folgender: Zuerst wurde der Zugring, in Fig. 83 mit 1 bezeichnet, betoniert, sodann die Rippenstucke 2 u 3 des ringes. Ganz entsprechend wurde auf der gegenubber liegenden Seite mit $2^{\prime}, 3^{\prime}, 4^{\prime}$ verfahren, um
eine vollständig symmetrische Belastung in jeder Meridian-Ebene zu erhalten. Die weitere Betonierungsfolge ist durch die Zahlen 5, 6, 7 und 8 angedeutet sowie entsprechend durch $5^{\prime}, 6^{\prime}, 7^{\prime}$ und $8^{\prime} ;$; auf diese Weise wurde zunächst das untere Ringstück der Kuppel geschlossen, sodann das
zweite, dritte und vierte Ringstuck bis zum Druckring.

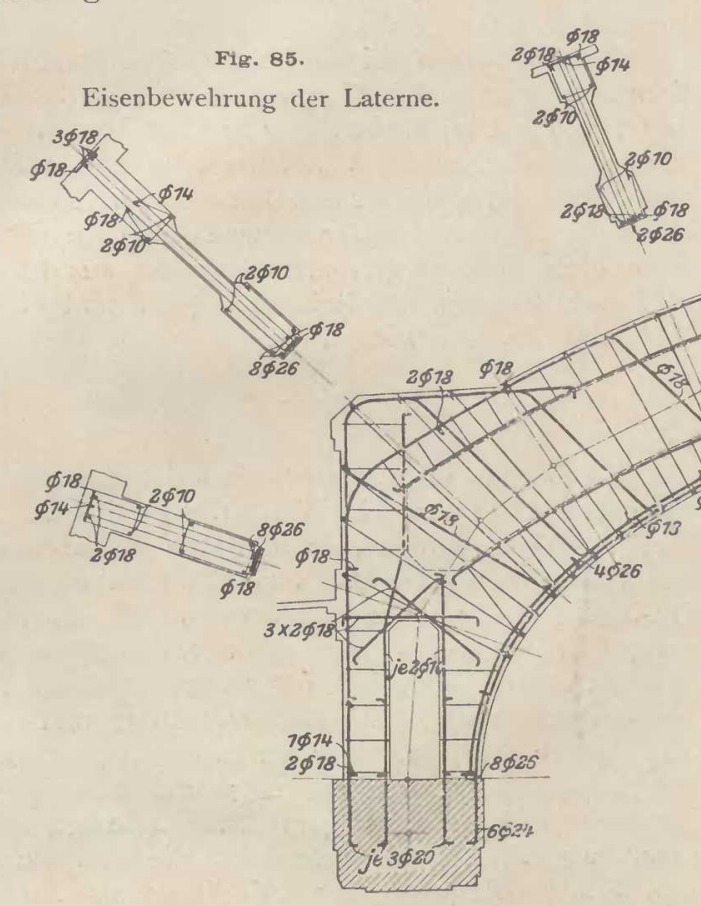

Dieser Vorgang bietet gegenüber der anderen einer Meridianrippe nach der anderen, den oroßen Vorteil, daß jedes einzelne Ringstück nach hinreichender Erhärtung sich selbst zu tragen imstande ist. Die obere Versteifungsrippe bildet jeweils Somit wurde ein Teil des Kraftstromes sobald als irgend möglich in das feste Widerlager, den
Zugring der Kuppel, geleitet und damit das LehrZugring der Kuppel, geleitet und damit das Lehr-
gerüst möglichst frühzeitig entlastet, so daß die gerüst möglichst frühzeitig entlastet, so daßs die
Formänderungen der Rüstung auf ein Mindestmaß beschränkt wurden. Die zum Betonieren des Zugringes erforderliche Zeit betrug 6 Arbeitstage, die Zeit für das Betonieren der übrigen Kuppe 18 Arbeitstag

Der für das Tragwerk der Kuppel verwendete Beton war verhältnismäßjig trocken, um ein gutes
Stampfen dieser hauptsächlich auf Druck beanspruchten Bauteile zu ermöglichen und ein willkürliches Herablaufen, wie es bei nassem Mortel ein- nen aus Eisenbeton hat die Verwendung trockenen 作 an die einzelnen Massen planmäfiger und zuver-

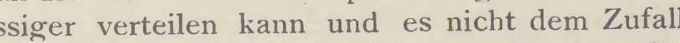
政

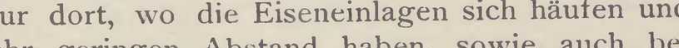
Verwendung von Vorsatzmörtel für die Ansichts2. B. bei der Querbahnsteighal Die unteren stark geneigten Teile der Kuppel-
Die

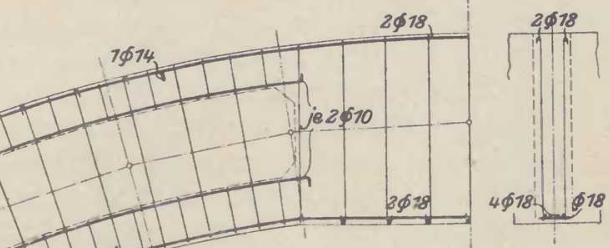

ippen wurden etwa bis zur Hälfte ihrer Länge gleicher Weise wi

Den Schluß bildete das Betonieren des Druckinges, "der innerhalb eines Arbeitstages vollendet werden konnte (siehe Fig. Alf. Um einen sorgippen zu erhalten, war bei der Herstellung des bbersten Teiles der Rippen eine vorübergehende enkrechte Schalung eingebaut worden, um das indringen des Betons in die Druclningschalung Suresen Stellen Auslinkungen angebracht. Die Ausführung der Laterne (Fig. 85) bot daier Rahmenbinder durch den Mittelturm der Kabelbahn hindurchgeführt werden mußte. Die Rüstung für die Laterne war auf dem Druckring der Kuppel durch Sprengwerke abgestut $z$, sonommen werden konnte, ohne durch die etwas verzögerte usführung der Laterne beeinträchtigt $\mathrm{zu}$ werden. Die senkrechten Pfosten der kräftigen LaternenUahmen waren im Druckring einbetoniert worden. belasten, wurde der mittlere volle Körper, der werksermaß̧en den Schlußsstein des ganzen Baudie senkrechten und wagerechten Teile der Rahmen. Wegen der zahlreichen Eiseneinlagen, der durch nischenförmige Aussparungen möglichst 
leicht gehalten waren, mußten diese Laternenschluß hieran wurden Wände und die Decken zwischen den 4 I techten bindern ausgeführt. Für die Aufbauten, die aus den senkrechten Säulen und den wagrechten
Decken bestanden, waren die Fenstersaulen auf dem Bauplatz in Schalkästen fabrikmäßigig hergedem Bauplatz in Schalkästen fabrikmäßig herge-
stellt und sodann mittels der Kabelbahn versetzt worden. Diese Arbeit ging sehr leicht vonstatten, sodaß das Versetzen je einer der kleineren Säulen nur 10 Minuten in Anspruch nahm.

für die Aufbauten waren zur brechten Decken isolierung und Schallwirkung Korkplatten vo $3 \mathrm{~cm}$ Stärke vorgesehen, die im Innenraum des Bauwerkes sichtbar blieben. Diese Platten wurde Beton der Desche ang verlegt und darauf der hatte sich ergeben, daß gung dieser Platten an den Decken nicht erforderich war, daß vielmehr die Haftung infolge des Ansaugens von Zent

en, sodaß der Firma Dyckerhoft \& Widmann An st dieser Erfolg auf das hante. Nicht zuletzt rbeiten der Bauverwaltung und der ausführenden as wäre nur zu inschen, daßs ein solches auf gegenFrzielung eines technetes Zusammenwirken Bauwerkes und im Interesse eines raschen Bay. fortganges allenthalben Anwendung finden würde. Hervorgehoben sei hier noch die Leistung der in Oppeln hat im Gefühl der tfabrik "Silesia ung so umfangreiche Versuchsreihen zur laufenPertere sie bisher ich Fabrikates erwiesen hat.

Wie bereits eingangs erwähnt, ist der archi(a) des Haupt tragwerkes ist von Stadtbauinspektor Dr.-Ing. Trauer aufgestellt worden. Die AusfühStudien und umeangreithen Bllen vorausgehenden gemeinsame Arbeit der Bauverwaltung und der ausführenden Firma. Der Aufgabe entsprechend waren an der Durcharbeitung und Bauleitung zwar lar seitens des Bauamtes die Bagt, und den Stadtbauinspektoren Schreiber als Architekten und Dr.Ing. Trauer als Ingenieur ob, die örtliche Bauleitung dem Bauassistenten Matthes Durchführung der Berechnungen der Bauverwat tung großen Anteil hat. Unter Leitung von Prof. Dr.-Ing. Gehler war mit der Entwurfsbearbeitung im technischen Bureau der Firma Dyckerhoff \&

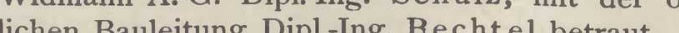

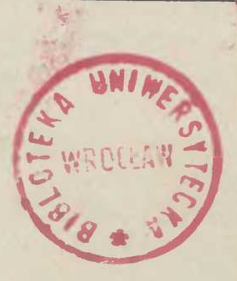

Gabinet
Sląsko-kutycki




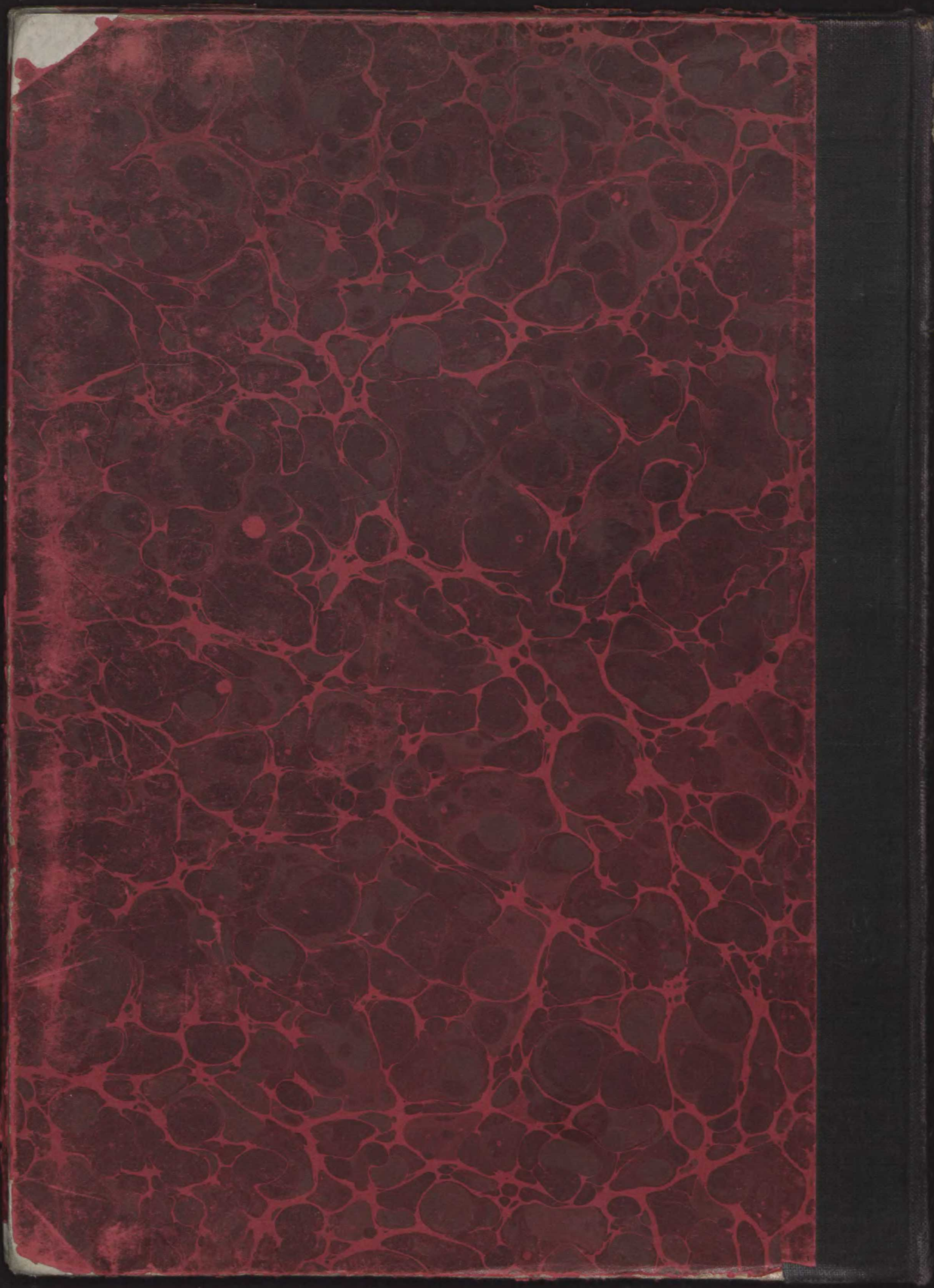

